University of Louisville

ThinkIR: The University of Louisville's Institutional Repository

Electronic Theses and Dissertations

$5-2014$

\title{
Expanding the frontier of gold-catalyzed cyclizations and rational ligand design.
}

Deepika Malhotra

University of Louisville

Follow this and additional works at: https://ir.library.louisville.edu/etd

Part of the Chemistry Commons

\section{Recommended Citation}

Malhotra, Deepika, "Expanding the frontier of gold-catalyzed cyclizations and rational ligand design." (2014). Electronic Theses and Dissertations. Paper 893.

https://doi.org/10.18297/etd/893

This Doctoral Dissertation is brought to you for free and open access by ThinkIR: The University of Louisville's Institutional Repository. It has been accepted for inclusion in Electronic Theses and Dissertations by an authorized administrator of ThinkIR: The University of Louisville's Institutional Repository. This title appears here courtesy of the author, who has retained all other copyrights. For more information, please contact thinkir@louisville.edu. 


\title{
EXPANDING THE FRONTIER OF GOLD- CATALYZED CYCLIZATIONS AND RATIONAL LIGAND DESIGN
}

\author{
By \\ Deepika Malhotra \\ B.S., Panjab University, Chandigarh, India, 2005 \\ M.S., Panjab University, Chandigarh, India, 2007
}

\author{
A Dissertation \\ Submitted to the Faculty of the \\ College of Arts and Sciences of the University of Louisville \\ in Partial Fulfillment of the Requirements \\ for the Degree of \\ Doctor of Philosophy
}

Department of Chemistry

University of Louisville

Louisville, Kentucky

May, 2014 



\title{
EXPANDING THE FRONTIER OF GOLD- CATALYZED CYCLIZATIONS AND RATIONAL LIGAND DESIGN
}

\author{
By \\ Deepika Malhotra \\ A Dissertation Approved on \\ April 16 ${ }^{\text {th }}, 2014$ \\ by the following Dissertation Committee:
}

Dr. Gerald B. Hammond

Dissertation Director

Dr. M. Cecilia Yappert

Dr. Christine Rich

Dr. Mark E. Noble

Dr. Mahendra K. Sunkara 


\section{DEDICATION}

This dissertation is dedicated to my respected father 'Sh. Pawan Kumar Malhotra', mother 'Smt. Kiran Malhotra' and my grandparents. 


\section{ACKNOWLEDGEMENTS}

I am extremely grateful to my research advisor, Dr. Gerald B. Hammond, for being an excellent and supportive mentor during my Ph.D. studies. I would like to thank him for his encouraging words on science and lessons on life, which I will remember forever. I express my deep gratitude towards the incredibly humble and intelligent Dr. Bo Xu, for guiding me throughout my doctoral work.

I would also like to thank the faculty members at University of Louisville Dr. M. Cecilia Yappert, Dr. Christine Rich, and Dr. Mark E. Noble of Department of Chemistry and Dr. Mahendra K. Sunkara of the Chemical Engineering Department, Speed School for their precious input, time, and for serving in my $\mathrm{Ph} . \mathrm{D}$. thesis defense committee. I am also thankful to Dr. Michael H. Nantz for his valuable suggestions and serving in my ORP and research committee. Also, I am thankful to Dr. Neal Stolowich who has been tremendously helpful in running NMR experiments and giving valuable advice towards solving the structure of complex organic molecules, especially natural products. I would like to acknowledge the computational studies support provided by Professor Ken Houk (UCLA) and Dr. Robert Paton (Oxford University, England, formerly a postdoctoral fellow in Professor Houk's group), described at length in Chapter 3 of this thesis. Also, I am grateful to Dr. Mark S. Mashuta for crystallographic support and Dr. William N. Richmond for technical help. 
I am thankful to various funding agencies; the National Science Foundation, the National Institute of Health, the University of Louisville for a graduate fellowship and teaching support, and the Institute of Molecular Diversity and Drug Design of the University of Louisville (IMD3) for a graduate fellowship and travel award.

During the course of my doctoral work, I was fortunate to be part of a dedicated research group: Dr. Bo Xu, Dr. LePing Liu, Dr. Weibo Wang, Dr. Zhuang Jin, Dr. Jose C. Aponte, Junbin Han, Manish Kumar, Shengzong Liang, Otome Elisa, Zhichao Lu, Mallory Durham, Ebule Rene and Andrew Flach. I believe our bond of friendship will always remain intact ever after I leave the lab. Last but not the least, I would like to thank the office staff in the Chemistry Department; Sherry Nalley, Syble Bullock, Aaron Howell, and Sabrina Haug, for being so supportive and helpful.

I am also indebted to Mari Mujica and Nidhi Shah for being such supportive friends and honest guides. I am thankful to all my friends at University of Louisville: Davinder, Gori, Rahul, Rohini, Radhika and Karina. It's a true blessing to have you all in my life. I am truly thankful to my Master's adviser Dr. TejVir Singh at Panjab University, Chandigarh, India for instigating the motivation and encouraging me to pursue higher studies in the field of organic chemistry.

I have a deep appreciation and sincere regards for my motivating parents, Pawan Kumar Malhotra and Kiran Malhotra without their prayers and support I would not have made it this far. I would also like to thank my wonderful husband, Neeraj Kumar for his unconditional love and being so supportive throughout. 


\section{ABSTRACT \\ EXPANDING THE FRONTIER OF GOLD-CATALYZED CYCLIZATIONS AND RATIONAL LIGAND DESIGN \\ Deepika Malhotra}

April 16 ${ }^{\text {th }}, 2014$

The focus of my dissertation work is to study the gold-catalyzed intramolecular and intermolecular cyclizations involving oxonium intermediates towards the application of synthetically interesting frameworks under ambient conditions and developing a rational approach for the effective catalyst design in gold catalysis. We explored the goldcatalyzed oxygen-transfer reactions of 2-alkynyl-1,5-diketones or 2-alkynyl-5-ketoesters to furnish five-membered rings bearing a quaternary carbon tethered to a carbonyl group. The detailed mechanistic investigation on the newly proposed intramolecular [4+2] cycloaddition mechanism was performed by means of isotopic experiments and quantum chemical calculations. The reactivity of alkynylenolate was investigated in the reactions of allenic ketones and vinyl ketones which led to versatile syntheses of 2-alkynyl-1,5-

diketones, 4-alkynyl-3-hydroxycyclohexones and 4-alkynylcyclohexenones. We also investigated the gold-catalyzed annulations of 2-alkynyl benzaldehyde with acyclic or cyclic vinyl ethers under very mild conditions, and successfully developed synthetically interesting dihydronaphthalenes, acetal-tethered isochromenes and bicyclo[2.2.2]octane 
derivatives often found in biologically active molecules and natural products. Although there have been numerous reviews and publications on new gold-catalyzed transformations, the development of new catalysts still relies on a hit-and-miss approach. Because the decay of the active cationic gold catalyst is the main reason for the high catalytic loading required for the majority of gold-catalyzed transformations, we developed a modular approach for effective catalyst design in gold catalysis. We discovered a new phosphine-based precatalyst that is broadly applicable and highly efficient—in the parts per million (ppm) range—at room temperature or slightly elevated temperatures $\left(<50^{\circ} \mathrm{C}\right)$. The ligand was preapared in one step from readily available starting materials. 


\section{TABLE OF CONTENTS}

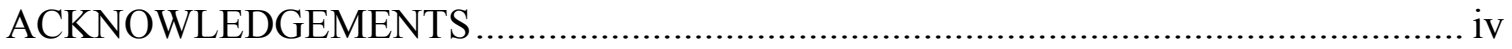

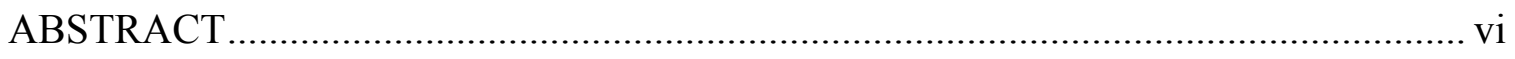

TABLE OF CONTENTS ....................................................................................... viii

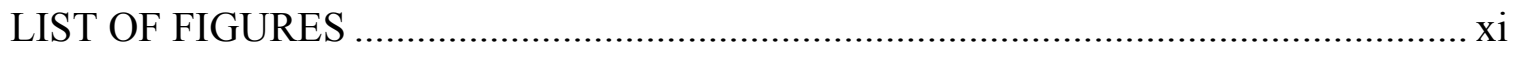

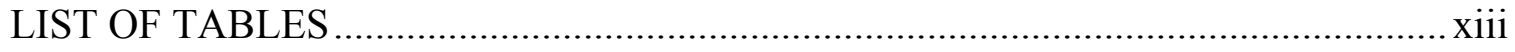

LIST OF SCHEMES................................................................................

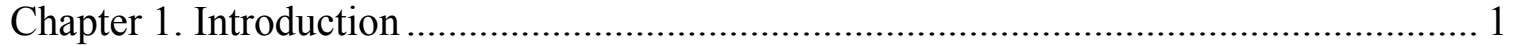

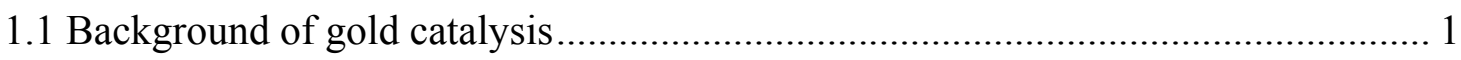

1.2 Gold-catalyzed reactions involving gold-oxonium intermediates ......................... 3

1.2.1 Carbonyl compounds as nucleophiles...................................................... 4

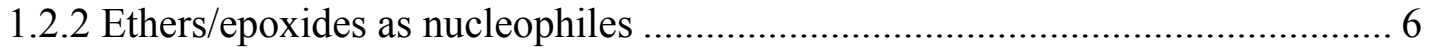

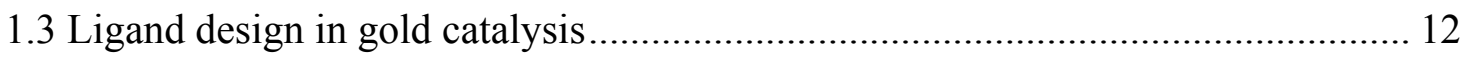

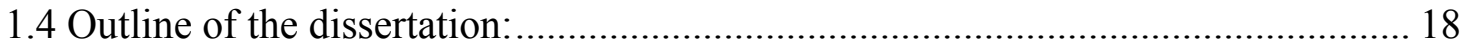

Chapter 2. Gold-Catalyzed Intramolecular Oxygen Transfer Reactions of 2-Alkynyl-1,5-

Diketones or 2-Alkynyl-5-Ketoesters ................................................................. 19

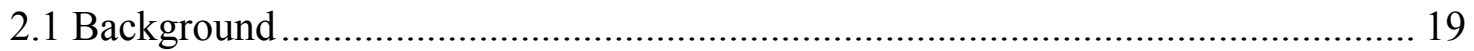

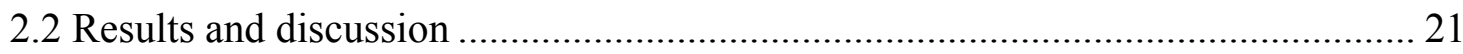

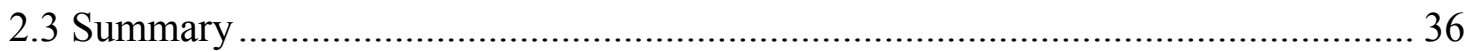

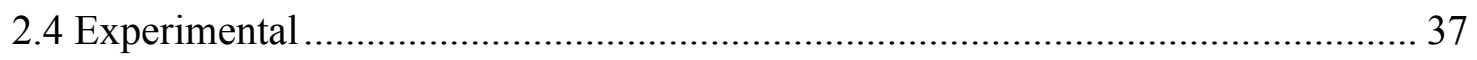

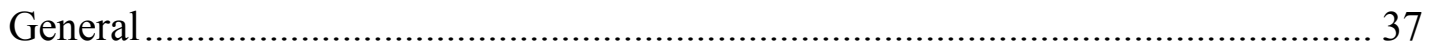

General procedure for gold-catalyzed oxygen transfer of 2-alkynyl-1,5-diketones 2-1

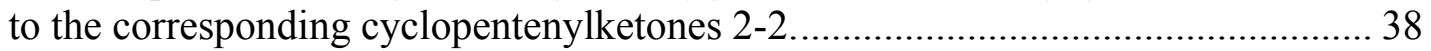

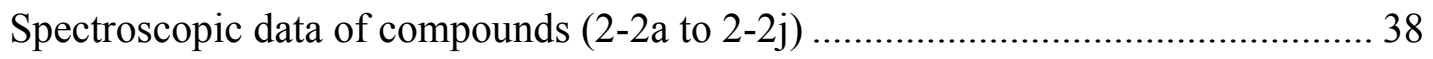

Chapter 3. Tandem Michael Addition/Aldol Reactions of Allenic Ketones ................... 52

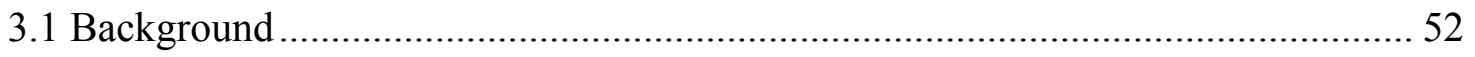


3.2 Results and discussion

3.2.1 Conformation analysis of transition states and NOESY effect of compound 3$4 \mathrm{~h}$. 59

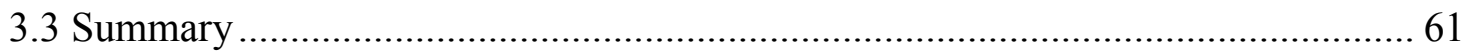

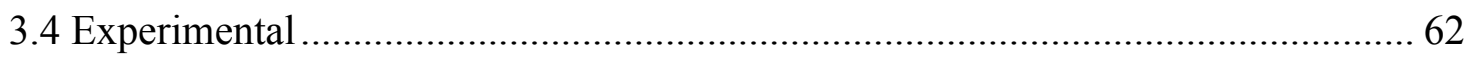

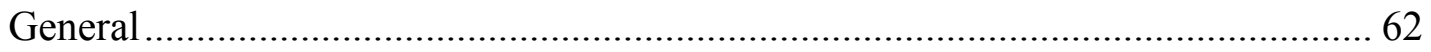

General procedure for the synthesis of 2-alkynyl 1,5-diketones 3-3:...................... 63

General procedure for the synthesis of 4-alkynyl-3hydroxycyclohexanones 3-4: ... 63

General procedure for the synthesis of 4-alkynylcyclohexenones 3-5:.................... 64

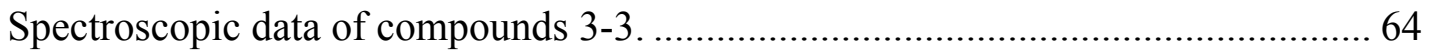

Spectroscopic data of compounds 3-4 ............................................................... 70

Spectroscopic data of compounds 3-5............................................................. 75

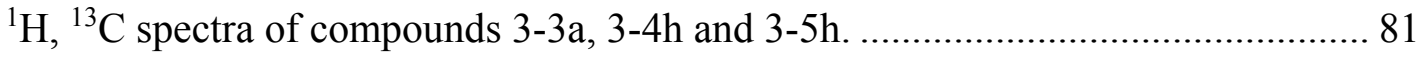

Chapter 4. Gold-Catalyzed Annulations of 2-Alkynyl Benzaldehydes ............................ 88

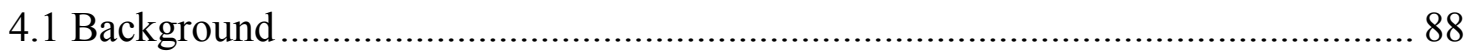

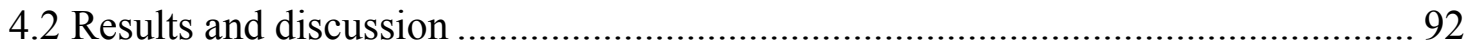

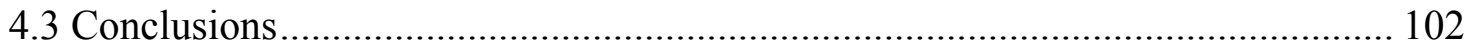

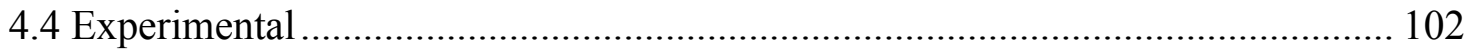

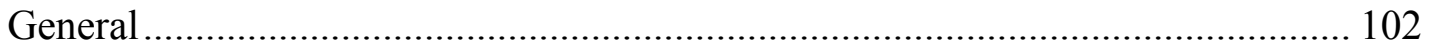

General procedure for the formation of dihydronaphthalenes (4-5):...................... 103

Spectroscopic data of compounds 4-5 ............................................................. 103

General procedure for the formation of isochromenes (4-6): ................................. 107

General procedure for the formation of bicyclo[2.2.2]octanes (4-7):.................... 111

General procedure for the formation of dimer (4-9):.............................................. 117

${ }^{1} \mathrm{H},{ }^{13} \mathrm{C}$ and ${ }^{19} \mathrm{~F}$ spectra of compound 4-5a,4-6a, 4-7a and 4-9............................ 119

Chapter 5. Rational Ligand Design For Gold Catalysis ................................................. 129

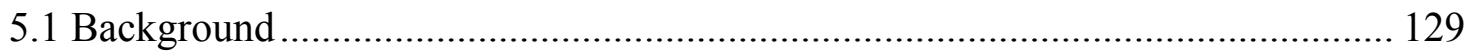

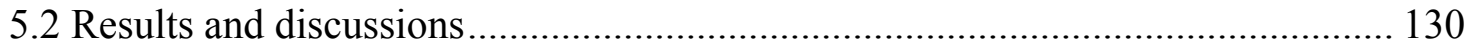

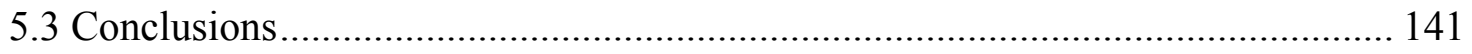

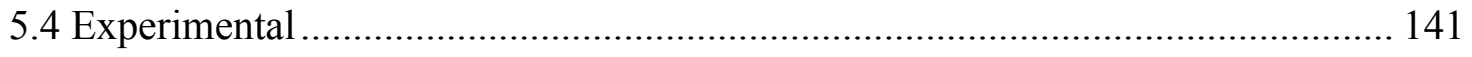




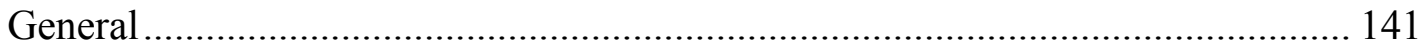

General procedure for the synthesis of Ligand L1 ......................................... 142

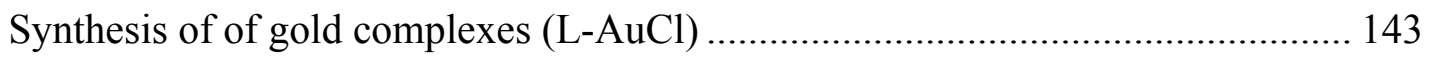

General procedures for model reactions ...................................................... 143

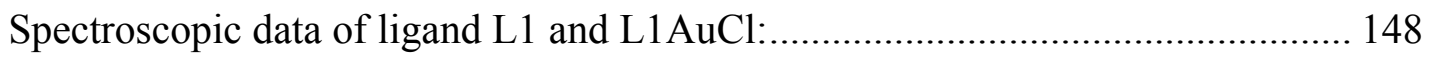

${ }^{1} \mathrm{H},{ }^{13} \mathrm{C}$ and ${ }^{19} \mathrm{~F}$ spectra of ligand $\mathrm{L} 1$ and array experiment. .............................. 150

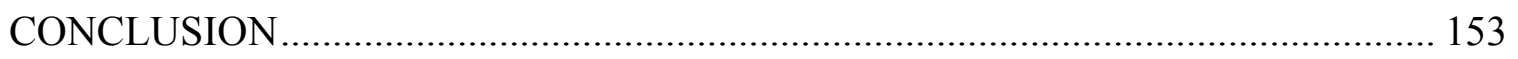

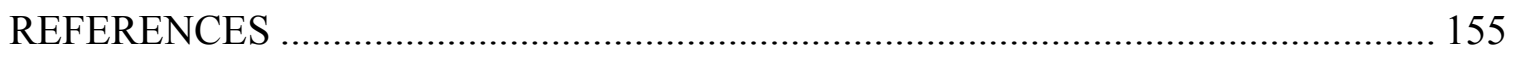

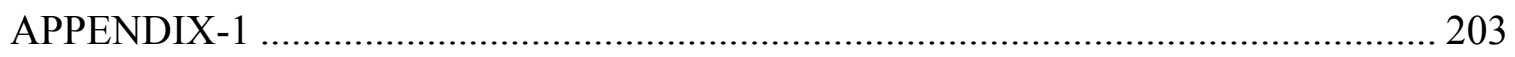

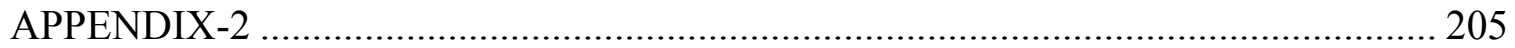

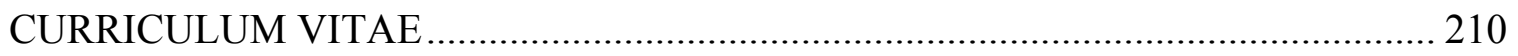




\section{LIST OF FIGURES}

Figure 1: Electronic effect in hydroamination reaction and ligand L1....................... 16

Figure 2: Above: B2PLYP/6-311+G(d,p)//B2PLYP/6-31G(d) (using LANL2DZ ECP on $\mathrm{Au})$ Relative energy Profile computed for Both [2+2] and [4+2] pathways for a model substrate. $\mathrm{R}_{1}, \mathrm{R}_{2}, \mathrm{R}_{3}, \mathrm{R}_{4}=\mathrm{Me}, \mathrm{Au}=\mathrm{AuCl}$. Below: B2PLYP/6-311+G(d,p)/B2PLYP/6$31 \mathrm{G}(\mathrm{d})$ computed stationary points for the model sytem. Energies relative to reactant 2-1Au. Bond forming/breaking distances in $\AA$.

Figure 3: Proposed mechanism and B2PLYP/6-311+G(d,p)/B2PLYP/6-31G(d) computed stationary points for the oxetene pathway. Energies relative to reactant 2-1. Bond breaking distance in $\AA$.

Figure 4: Proposed mechanism and B2PLYP/6-311+G(d,p)/B2PLYP/6-31G(d) computed stationary points for the oxetene pathway. Energies relative to reactant $2-1$. Bond breaking distance in $\AA$. 32

Figure 5: Proposed mechanism and B2PLYP/6-311+G(d,p)/B2PLYP/6-31G(d) computed stationary points for the 7-endo-dig pathway. Energies relative to reactant 2-1. Bond breaking distance in $\AA$. 33

Figure 6: Proposed mechanism and B2PLYP/6-311+G(d,p)/B2PLYP/6-31G(d) computed stationary points for the 6-exo-dig pathway. Energies relative to reactant 1 . Bond breaking distance in $\AA$. 35

Figure 7: B2PLYP/6-311+G(d,p)/B2PLYP/6-31G(d) (hydrogen-deleted) transition structures for intramolecular and intermolecular cyclization of a ketone onto a Aucoordinated alkyne. Bond distances in $\AA$ with associated Wiberg bond indices italicized. Activation energies relative to Au-coordinated alkyne in $\mathrm{kcal} / \mathrm{mol}$. 36

Figure 8: ORTEP-3 diagram of bicyclo[2.2.2] octane derivative 4-7a, showing $40 \%$ probability ellipsoids. $\mathrm{H}$ atoms are shown as small spheres of arbitrary radii. Selected bond lengths $[\AA]$ and angles $\left[{ }^{\circ}\right]$ : O1-C2, 1.4285(17); O1-C3, 1.4380(18); O2-C9, 1.4419(18); O2-C10, 1.4338(16); O3-C17, 1.2100(18); O2-C10-C1, 111.15(11); O1-C2C3, 111.78(11); C2-C1-C10, 104.06(11).

Figure 9: ORTEP-3 diagram of product $9 \mathrm{~b}$, showing one diastereoisomer of the racemic mixture at $40 \%$ probability ellipsoids. $\mathrm{H}$ atoms are shown as small spheres of arbitrary radii. Selected bond lengths $[\AA]$ and angles $\left[{ }^{\circ}\right]$ : O1-C7, 1.3795(15); O1-C15, 1.4447(15); O2-C17, 1.2157(15); O3-C30, 1.2117(16); C15-C16, 1.5531(17); C7-O1-C15, 114.70(9); O1-C7-C8, 121.07(11); O1-C15-C16, 106.72(9). 101

Figure 10: o-Biphenyl group enhanced protodeauration. 133 
Figure 11. Two categories of phosphine ligands. .................................................... 134

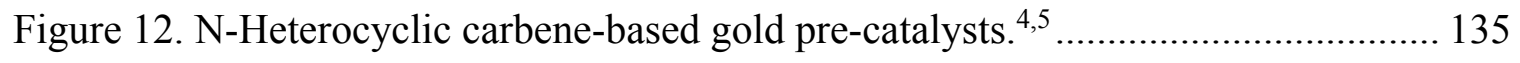

Figure 13. Ligand design for gold catalysis....................................................... 135 


\section{LIST OF TABLES}

Table 1: Gold-catalyzed intramolecular oxygen transfer reactions of 2-alkynyl-1,5-

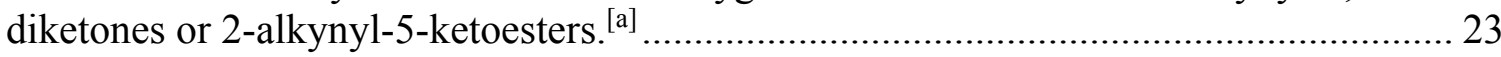

Table 2. Base-mediated reactions of allenic ketone 3-1a with methyl vinyl ketone. ${ }^{\mathrm{a}}$...... 55

Table 3: TBAF-mediated Michael addition of allenic ketones 3-1 to vinyl ketones 3-2. ${ }^{[a]}$

Table 4. Tandem Michael addition/aldol reaction of allenic ketones 3-1 with vinyl ketones 3-2 at low temperature. ${ }^{[a]}$

Table 5: Tandem Michael addition/aldol condensation of allenic ketones 3-1 with vinyl ketones 3-2 at room temperature. ${ }^{[a]}$

Table 6: Scope of the gold-catalyzed annulation of 2-alkynyl benzaldehyde with vinyl ether. ${ }^{\mathrm{a}}$.

Table 7: Gold-catalyzed annulation of 2-alkynyl benzaldehyde with vinyl ether in the presence of alcohol. ${ }^{2}$

Table 8: Gold-catalyzed annulation of 2-alkynyl benzaldehyde with cyclic vinyl ether. ${ }^{a} 98$

Table 9: Ligand effects in the protodeauration of vinyl gold complex 1

Table 10. Relative rates of hydroamination for various ligands 136 


\section{LIST OF SCHEMES}

Scheme 1: Selected examples of gold-catalyzed cyclizations. 2

Scheme 2: A: Schematic view of the molecular orbital energies for hypothetic Au compounds before and after relativistic considerations. B: Calculated relativistic contraction of $6 \mathrm{~s}$ orbital.

Scheme 3: General mechanism involved in gold catalysis.............................................. 3

Scheme 4: Oxonium intermediates involved in gold catalysis.......................................... 3

Scheme 5: Cycloisomerization of propargyl ketone 1-1.............................................. 4

Scheme 6: Gold-catalyzed synthesis of susbstituted cyclic enones 1-10 polycyclic enones $1-11$. 5

Scheme 7. Tandem heterocyclization and Nazarov reaction for fused furan carbocyles 113 and cycloisomerization of $\beta$-alkynyl $\beta$-ketoesters $1-14$................................................ 6

Scheme 8. Chemoselective synthesis of benzofurans $1-17$............................................. 7

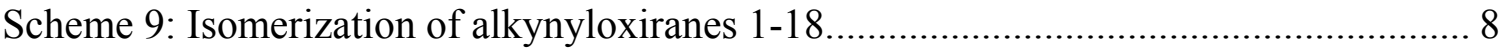

Scheme 10: Cycloisomerization and tandem cyclization/Friedel-Crafts reactions of

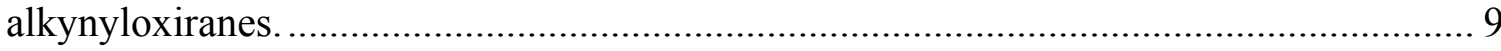

Scheme 11: Intramolecular reaction of allyloxyphenylpropynone 1-24 ......................... 10

Scheme 12: Strategy for intramolecular-oxygen-transferred reactions. ........................... 10

Scheme 13. Gold-catalyzed benzannulations and our aim. .......................................... 11

Scheme 14: Ligand effect in hydroamination of allenes 1-27 and cycloisomerization of propargyl amines 1-29...................................................................................... 13

Scheme 15: Intramolecular addition of diol to alkyne with highly active mononuclear

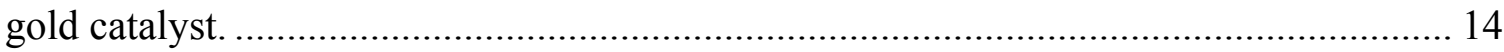

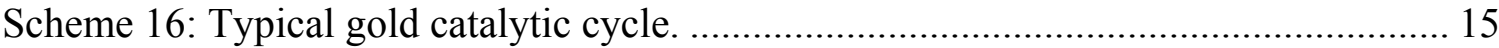

Scheme 17. Lewis or brønsted acid- and gold-catalyzed intermolecular or intramolecular alkyne-carbonyl metathesis....................................................................................... 20

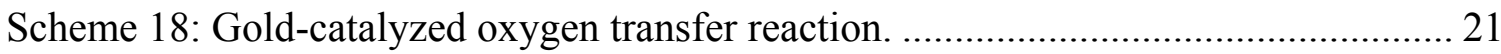

Scheme 19: Selected examples of natural products having a carbonyl tethered quaternary carbon on a five-membered ring. 22 
Scheme 20: ${ }^{18} \mathrm{O}$ isotopic labeling experiment for mechanistic studies............................ 25

Scheme 21: Synthesis of substrate 2-1b- ${ }^{18} \mathrm{O}$ and its ${ }^{13} \mathrm{C}$ NMR spectrum......................... 25

Scheme 22: ${ }^{18} \mathrm{O}$ isotopic experiment of alkynyldiketone and its ${ }^{13} \mathrm{C}$ NMR spectrum...... 26

Scheme 23: Synthesis of substrate 2-1o- ${ }^{18} \mathrm{O}$ and its ${ }^{13} \mathrm{C}$ NMR spectrum....................... 27

Scheme $24:{ }^{18} \mathrm{O}$ isotopic experiment of alkynylketoester and its ${ }^{13} \mathrm{C}$ NMR spectrum. .... 27

Scheme 25: Reactivity of alkynylenolates generated from allenoates or propargylic esters.

Scheme 26: Control transformations between compounds 3-3a, 3-4a and 3-5a............... 56

Scheme 27. Conformation analysis of transition states and NOESY effect of compound 3-4h.

Scheme 28: Conformation analysis of transition states and NOESY effect of compound $3-4 \mathrm{i}$.

Scheme 29: Selected examples of recently developed gold catalysts.............................. 89

Scheme 30: Yamamoto's group pioneered the gold or copper-catalyzed annulation of 2alkynyl benzaldehyde with alkyne, alkene, vinyl ether, or aldehyde. $\mathrm{R}_{4}$ is $\mathrm{H}$ or alkyl in B.

Scheme 31: New developments on gold-catalyzed annulations of 2-alkynyl benzaldehydes with vinyl ethers.

Scheme 32: Selected examples of biologically active compounds containing dihydronaphthalene, isochromene, or bicyclo[2.2.2] octane substructures.

Scheme 33: Proposed mechanisms for the gold-catalyzed annulations of 2-alkynyl benzaldehyde with vinyl ether.

Scheme 34: Proposed mechanism for the gold-catalyzed annulation of 2-alkynyl benzaldehyde with cyclic vinyl ether.

Scheme 35: Gold-catalyzed homo-dimerization of 2-alkynyl benzaldehyde and the proposed mechanism.

Scheme 36. Type I and Type II gold-catalyzed reactions............................................. 131

Scheme 37. Use of $\mathrm{L} 1-\mathrm{AuCl}$ in $\mathrm{X}-\mathrm{H}(\mathrm{X}=\mathrm{N}, \mathrm{O})$ addition to alkynes............................ 138

Scheme 38. C-H additions to alkynes (C-C bond formations). ...................................... 139

Scheme 39. Enyne and allenone cycloisomerization...................................................... 140 


\section{CHAPTER 1. INTRODUCTION}

\subsection{Background of gold catalysis}

In the past two decades, homogeneous gold catalysis has proven to be a constructive tool for the synthesis of versatile organic frameworks. It has emerged as a hot research field and this is reflected by the exponential surge in the number of publications on goldcatalyzed transformations including cycloisomerizations, carbocyclizations, cycloadditions, and heteroatom addition to unsaturated C-C bonds to form myriad products of synthetic importance. ${ }^{1-17}$ For example, gold-catalyzed cyclizations provide an efficient way for the construction of wide range of heterocycles ${ }^{18}$ as well as carbocycles. ${ }^{19}$ Selected examples include cycloisomerization of propargyl ketones by Hashmi ${ }^{20}$, cyclization of $\alpha$-hydroxy allenes by $\mathrm{Krause}^{21}$, benzannulation of $o$ alkynylbenzaldehyde by Yamamoto ${ }^{22}$ and double cyclization of 1,5-enynes by Kozmin, ${ }^{23}$ as shown in Scheme 1.

Unlike other transition metal catalyzed reactions, most of the gold-catalyzed transformations occur at ambient conditions and can proceed well in the presence of air and moisture, making this chemistry greener and easier. The catalytic properties of both $\mathrm{Au}$ (III) and $\mathrm{Au}$ (I) complexes have been exploited in a plethora of organic transformations and many unique features are associated with its reactivity. 
The unique reactivity of gold catalysts could be explained by the relativistic effect. ${ }^{24-26}$ As a result of the relativistic effect, the radius of the $6 \mathrm{~s}$ orbital is contracted and $5 \mathrm{~d}$ orbitals expand as displayed in Scheme 2. Therefore, the orbital contraction leaves the LUMO in a low-lying level of energy, which accounts for the higher reactivity observed.

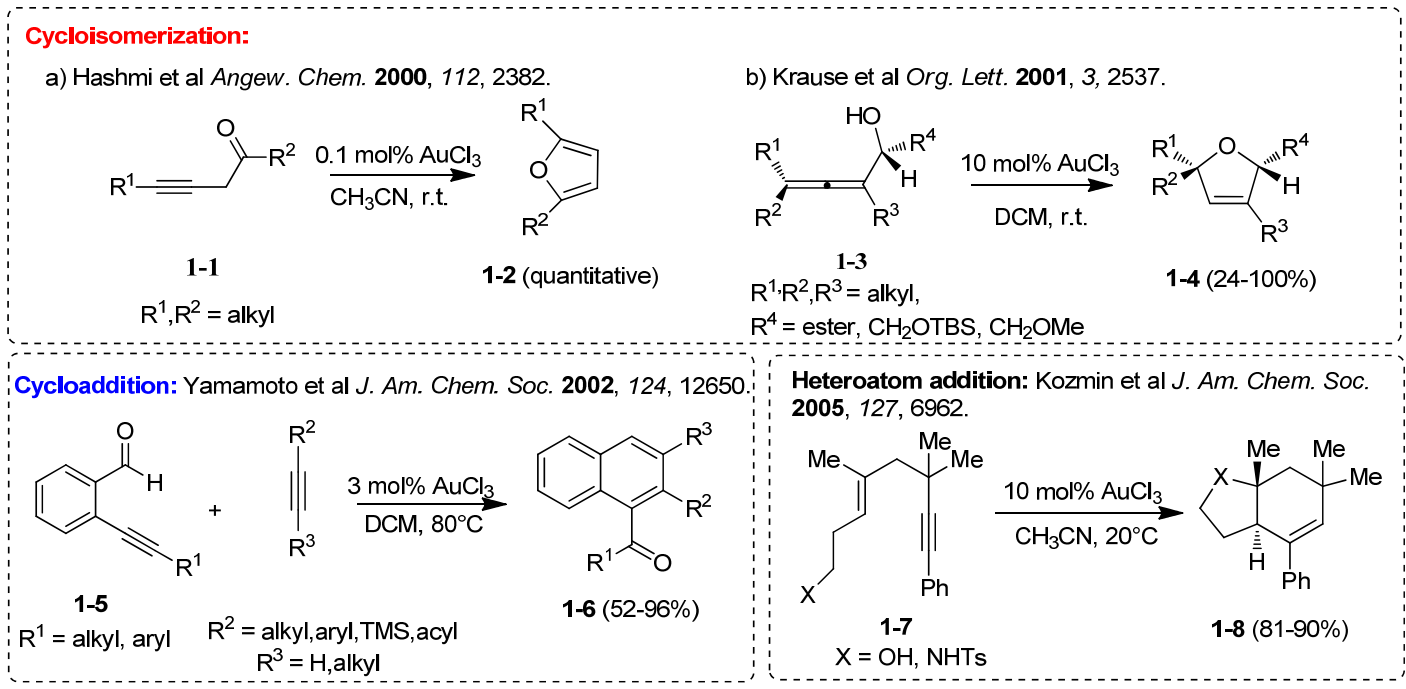

Scheme 1: Selected examples of gold-catalyzed cyclizations.

A

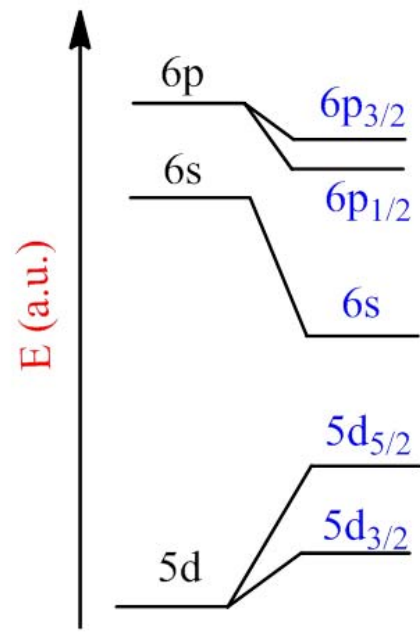

Non-relat.

Relat.
B

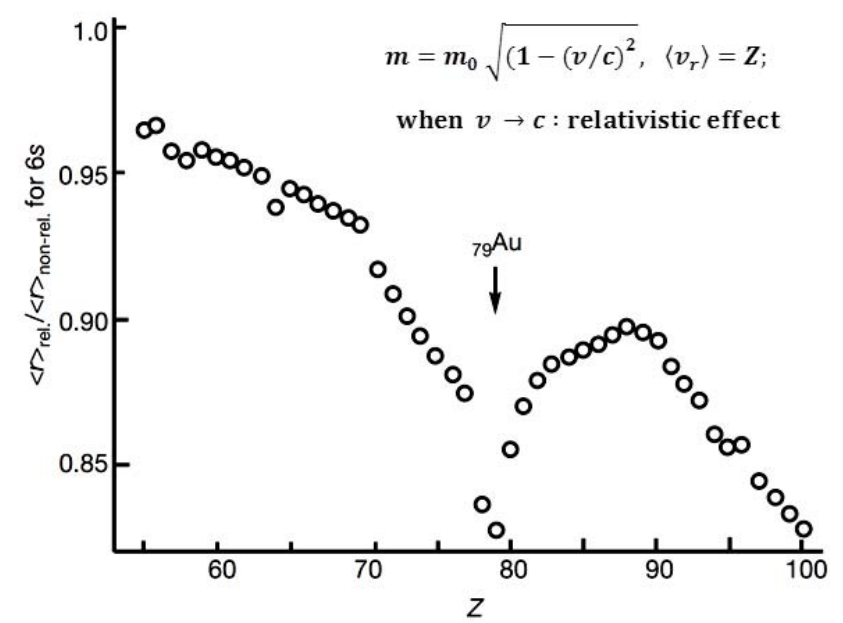

Scheme 2: A: Schematic view of the molecular orbital energies for hypothetic Au compounds before and after relativistic considerations. B: Calculated relativistic contraction of $6 \mathrm{~s}$ orbital. 
From a mechanistic standpoint, the coordination of gold catalyst with substrates such as alkynes, alkenes, and allenes enhances their electrophilicity. Subsequently, a nucleophile attacks the activated C-C multiple bond either inter- or intramolecularly followed by electrodeauration to selectively form carbocycles or heterocycles as displayed in Scheme 3.

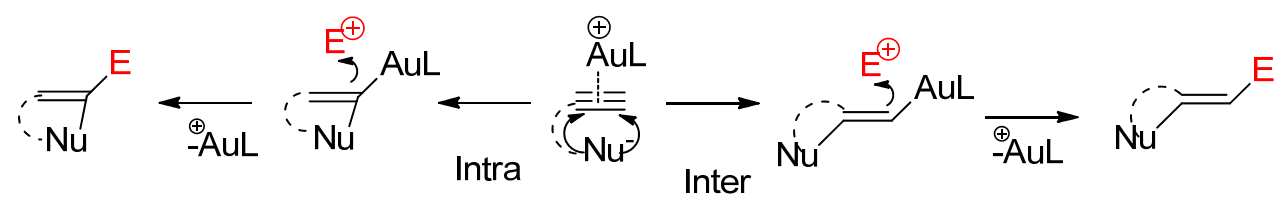

Scheme 3: General mechanism involved in gold catalysis.

\subsection{Gold-catalyzed reactions involving gold-oxonium intermediates}

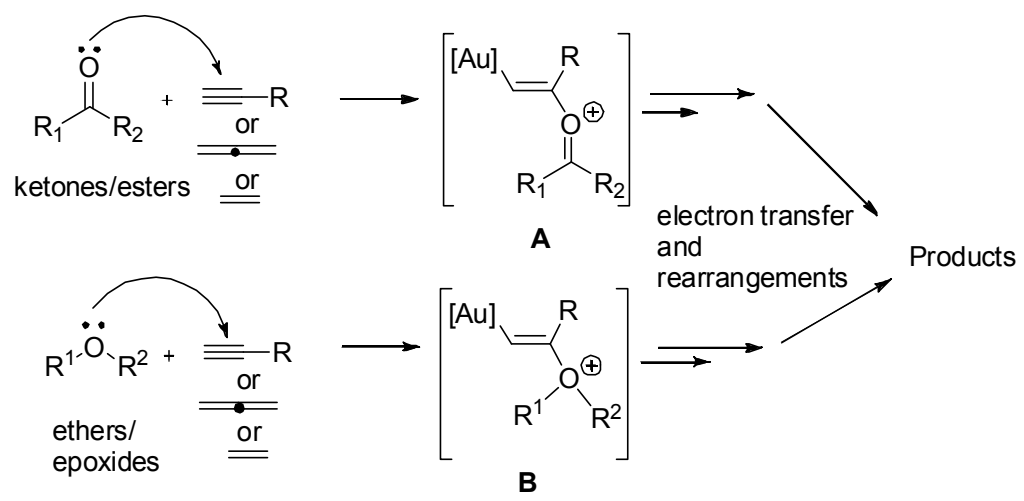

\section{Scheme 4: Oxonium intermediates involved in gold catalysis.}

In general, when oxygen containing nucleophiles such as carbonyl compounds (aldehydes/ketones/esters) attack gold activated multiple bonds they generate an oxonium intermediate such as $\mathbf{A}$. This oxonium intermediate $\mathbf{A}$, being a highly energetic species, will undergo electron transfers/rearrangement/protodeauration to form corresponding products (Scheme 4). Similarly, when nucleophiles such as ethers/epoxides attack gold activated multiple bonds, an oxonium intermediate such as $\mathbf{B}$ is generated, which may 
further undergo several rearrangements/transformations to furnish synthetically interesting products (Scheme 4).

\subsubsection{Carbonyl compounds as nucleophiles}

An early example of intramolecular cycloisomerization is the gold-catalyzed reaction of propargyl ketones $\mathbf{1 - 1}$ reported by Hashmi et al ${ }^{20}$ in 2000 (Scheme 5). The coordination of gold (III) activates the alkyne moiety which is followed by nucleophilic attack of the carbonyl oxygen to form gold oxonium intermediate $\mathbf{1 - 2 A}$, which subsequently undergoes proton transfer/protodemetallation to afford furan substrates.

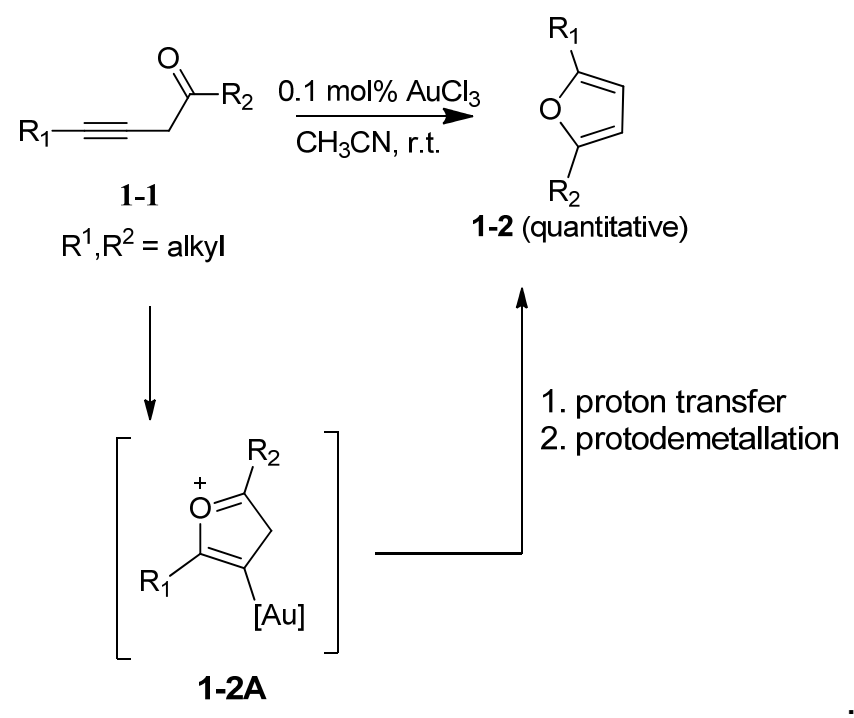

\section{Scheme 5: Cycloisomerization of propargyl ketone 1-1.}

In 2007, Yamamoto and coworkers reported the gold-catalyzed synthesis of substituted cyclic enones 1-10 from tethered alkynyl ketones 1-9, in good yields ${ }^{17}$ (Scheme 6). In this case, the gold activated alkyne is attacked by the carbonyl group to form an oxonium intermediate 1-9A, which subsequently undergoes electron transfer and ring opening to furnish cyclic enones. A year later, the same authors developed a gold-catalyzed cascade reaction towards the synthesis of polycyclic enones $\mathbf{1 - 1 1 ^ { 2 7 }}$. In their tandem metathesis 
and Nazarov reaction, the oxonium intermediate undergoes rearrangements followed by sequential Nazarov reaction to form polycyclic enones 1-11.

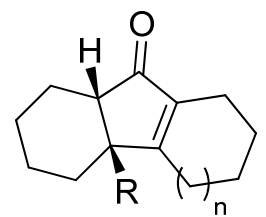

1-11

$33-85 \%$

$\mathrm{n}=0,1,2,3$

$\mathrm{R}=\mathrm{Et}$

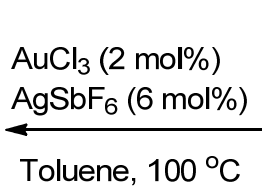

Toluene, $100^{\circ} \mathrm{C}$

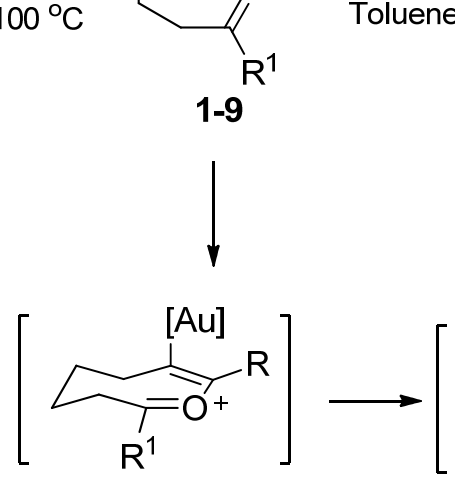

1-9A

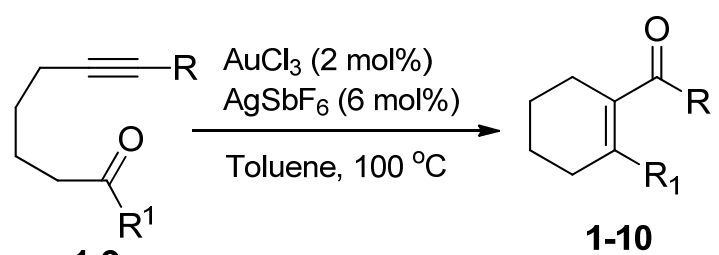

$54-91 \%$

$\mathrm{R}=\mathrm{C}_{6} \mathrm{H}_{5}, 4-\mathrm{Me}-\mathrm{C}_{6} \mathrm{H}_{4}, 4-\mathrm{CO}_{2} \mathrm{Me}-\mathrm{C}_{6} \mathrm{H}_{4}$ $4-\mathrm{CF}_{3}-\mathrm{C}_{6} \mathrm{H}_{4}, \mathrm{Pr}$ $\mathrm{R}^{1}=\mathrm{Et}$

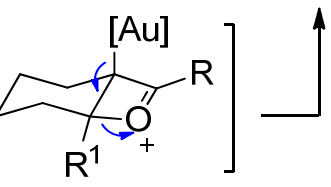

$1-9 B$

\section{Scheme 6: Gold-catalyzed synthesis of susbstituted cyclic enones 1-10 polycyclic enones 1-11.}

Manoharan and coworkers ${ }^{28}$ also reported a tandem heterocyclization/Nazarov reaction in 2011 that furnished substituted fused furan carbocycles 1-13 (Scheme 7). The oxonium intermediate 1-12A could be trapped using two equivalents of methanol that clearly revealed the heterocycle formation was triggered by a Nazarov reaction to form the corresponding fused furan carbocyles, using dichloromethane as solvent. The intermediate 1-12A undergoes Nazarov reaction to form intermediate 1-12B, which eventually form furan carbocyles. In the same year, Moran and coworkers ${ }^{29}$ reported a strategy for the synthesis of substituted furans by the cycloisomerization of $\beta$-alkynyl $\beta$ ketoesters 1-14 (Scheme 7). In this cycloisomerization, the intramolecular attack of the oxygen of a ketone on the activated alkyne formed the oxonium intermediate $\mathbf{1 - 1 5 A}$, which underwent protodeauration and isomerization to yield substituted furans. 
<smiles>[R]C#C/C(=C\c1ccccc1)C(=O)C(=C)OCC</smiles>

1-12

$\mathrm{R}=$ Phenyl, $p$-Methoxyphenyl $p$-Nitrophenyl, $p$-Fluorophenyl,

1-Cyclohexenyl, $n$-Butyl, $\alpha$-Naphthyl<smiles>[R]C1=C([Al]C)C(=CP)C(C(=C)OCC)=C1[Hg]C</smiles>

$1-12 A$<smiles>[R]c1cc2c(o1)C(=O)CC2[PbH]</smiles>

$51-85 \%$ yield

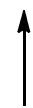<smiles>[R]C1=C([Al]CCCCC)C2OC1C(=O)CC2c1ccccc1</smiles>

1-12B<smiles>[R]C#CCC(C([R])=O)C([R])=O</smiles>

1-14

$\mathrm{R}^{1}=\mathrm{Me}, \mathrm{Et}, \mathrm{Ph}, p-\mathrm{NO}_{2} \mathrm{Ph}$

$\mathrm{R}^{2}=\mathrm{Me}, \mathrm{Et}$

$\mathrm{R}_{3}=\mathrm{Ph}, \mathrm{H}, p$-ClPh, $p$-OMePh, Et, thiophene<smiles>[R3]Cc1cc(C(=O)O[R2])c([R])o1</smiles>

1-15

$32-99 \%$ yield<smiles>[R]OC(=O)C1C=CC(CC)C1=C(CC)CC</smiles>

1-15A

Scheme 7. Tandem heterocyclization and Nazarov reaction for fused furan carbocyles 1-13 and cycloisomerization of $\beta$-alkynyl $\beta$-ketoesters 1-14.

\subsubsection{Ethers/epoxides as nucleophiles}

In 2013, Hashmi et al. ${ }^{30}$ discovered a chemoselective one-pot synthesis of benzofuran 117 from alkynyl-7-oxabicyclo[4.1.0]heptan-2-ones 1-16 (Scheme 8). The carbonyl oxygen acts as nucleophile to the gold activated triple bond to generate the fused ring 
oxonium intermediate 1-17A, which subsequently undergoes 1,2-hydride shift followed by sequential rearrangements to give 1-17B which then eliminates methanol and water to yield substituted benzofurans.

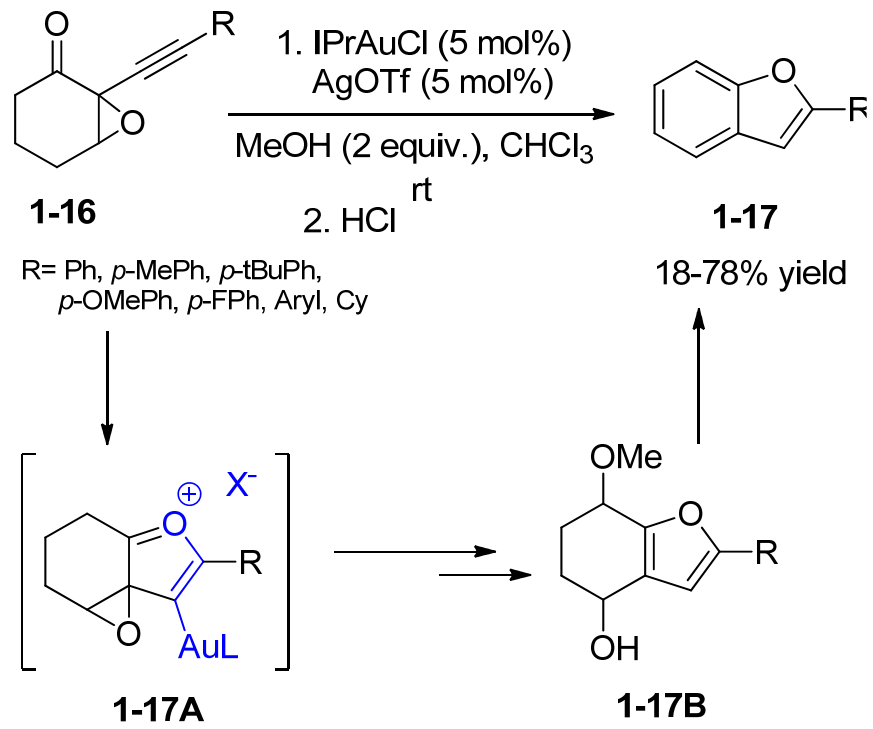

Scheme 8. Chemoselective synthesis of benzofurans 1-17.

In 2004 Hashmi et al. ${ }^{31}$ reported a gold-catalyzed isomerization of alkynyloxiranes 1-18 to the corresponding furan derivates 1-19 (Scheme 9). In the mechanism, the gold catalyst activates the triple bond followed by nucleophilic attack of epoxide oxygen at the distal position of the activated alkyne to generate five membered oxonium intermediate 1-18B, which then undergoes deprotonation and proto-demetallation to yield substituted furans. 


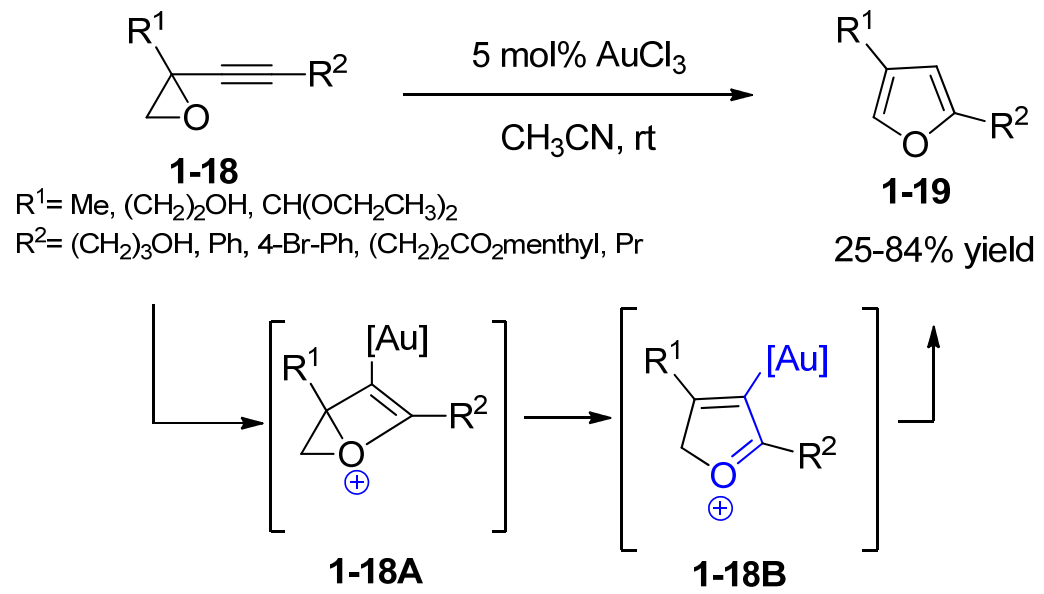

Scheme 9: Isomerization of alkynyloxiranes 1-18.

An efficient cycloisomerization of alkynyloxiranes 1-20 was developed by Liang et al. ${ }^{32}$ in 2007 to afford 2,5-disubstituted furans 1-21 (Scheme 10). The gold activated alkyne is attacked by an epoxide oxygen acting as nucleophile to form oxabicyclo oxonium ion intermediate 1-20A, which subsequently undergoes protonation to give 2,3-dihydrofuran, followed by reductive elimination to yield 2,5-disubstituted furans. Two years later, in 2009, Liang and coworkers ${ }^{33}$ reported a tandem cyclization/Friedel-Crafts reaction to furnish furan derivatives in good yields 1-23 (Scheme 10). Plausible mechanism for this reaction involves the nucleophilic attack of epoxide oxygen on the gold-coordinated alkynyl substrate, followed by protonation, reductive elimination and intermolecular Friedel-Crafts type reactions to generate substituted furans. 

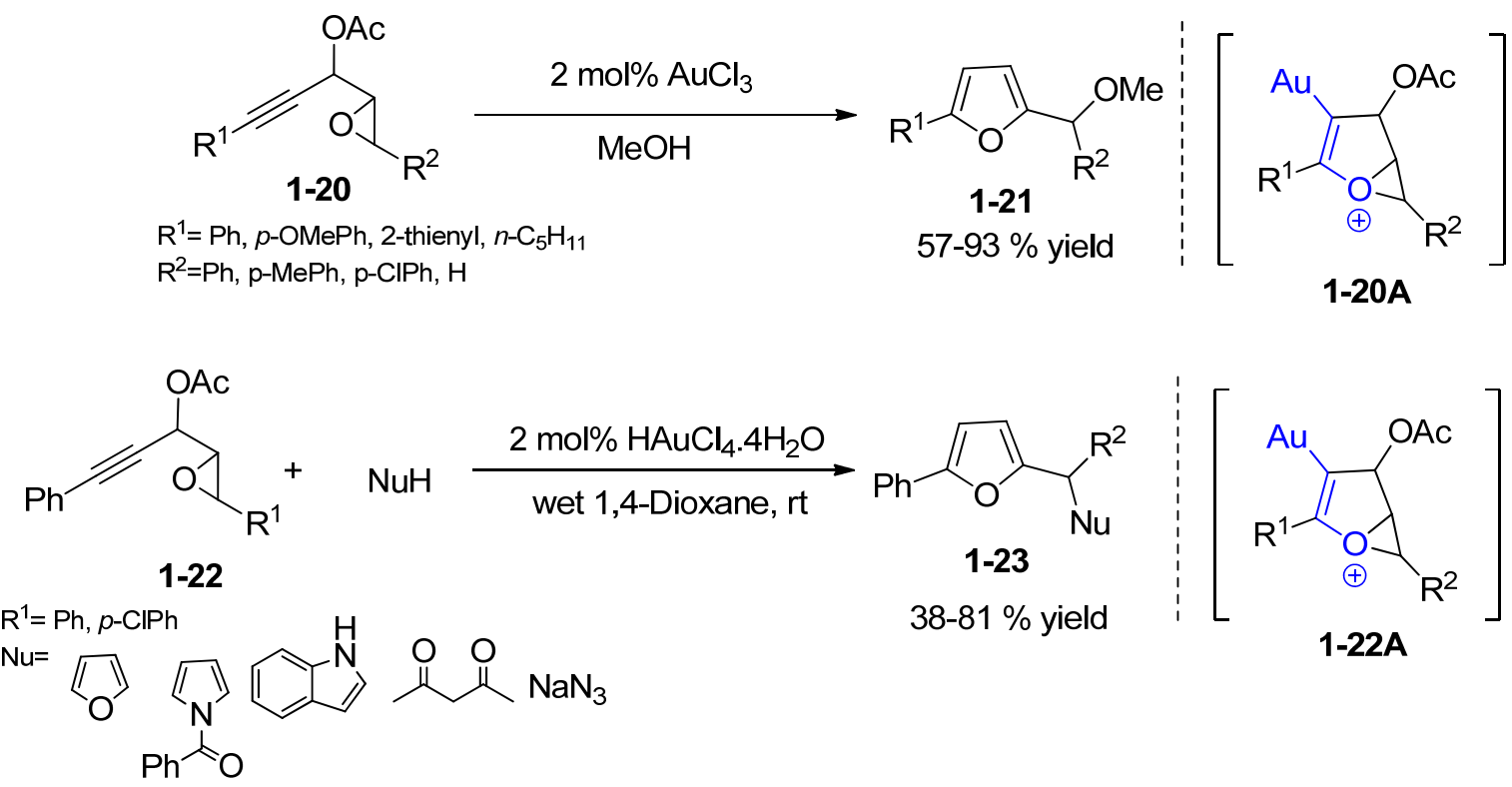

Scheme 10: Cycloisomerization and tandem cyclization/Friedel-Crafts reactions of alkynyloxiranes.

In 2011, Goualt and coworkers ${ }^{34}$ developed a gold-catalyzed intramolecular reaction of allyloxyphenylpropynone 1-24 to afford chromones 1-25 in moderate yields (Scheme 11). The gold activated alkyne moiety is attacked by a lone pair on oxygen to generate sixmembered oxonium ion intermediate $\mathbf{1 - 2 4 A}$, which then undergoes substituent transfer via carbodemetallation to form substituted chromone derivatives.
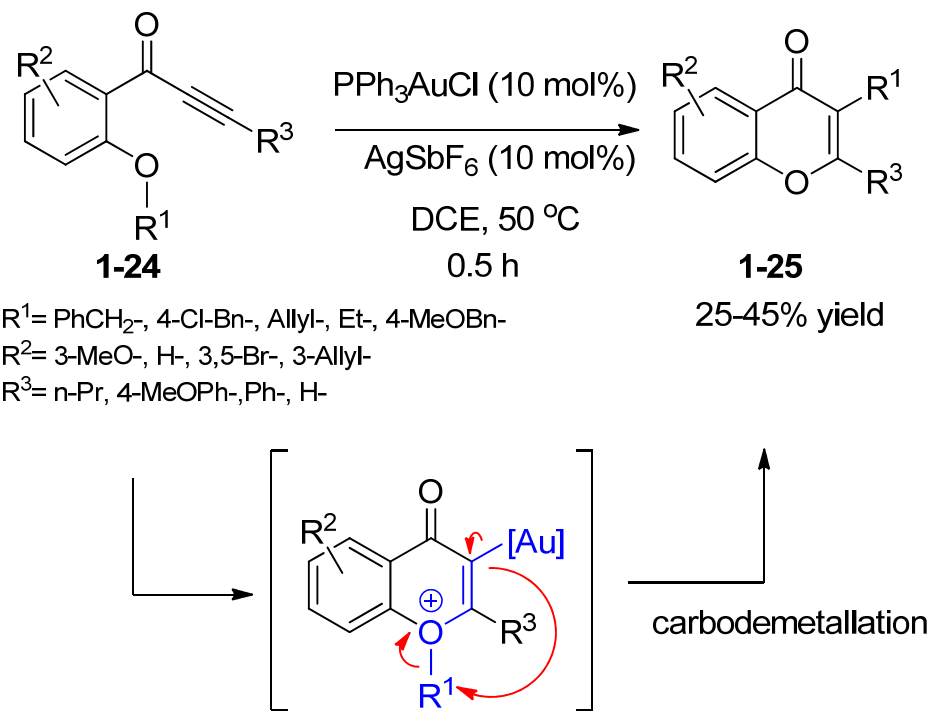

1-24A 


\section{Scheme 11: Intramolecular reaction of allyloxyphenylpropynone 1-24.}

While probing the reactivity of the alkynylenolate (Scheme 12) we discovered the synthesis of 2-alkynyl-1,5-dicarbonyl derivatives 3-3 from the Michael addition of activated allenes to electron-deficient olefins, ${ }^{35}$ the detailed synthesis of which will be described in Chapter 3. Since two carbonyls and a carbon-carbon triple bond had been installed in the substrate, we envisioned that oxonium intermediate (Scheme 12) containing a quaternary propargylic carbon $\mathbf{J}$ could be generated from the 2-alkynyl-1,5dicarbonyl 3-3 as a result, Path A would be blocked and an alternate Path B could provoke novel transformations of the oxonium intermediate to generate complex ring systems (Scheme 12). Gratifyingly, an intramolecular-oxygen-transferred reaction yielded highly substituted five-membered rings bearing a quaternary carbon tethered to a carbonyl group and the detailed scope and mechanistic details of this transformation will be discussed in Chapter 2.

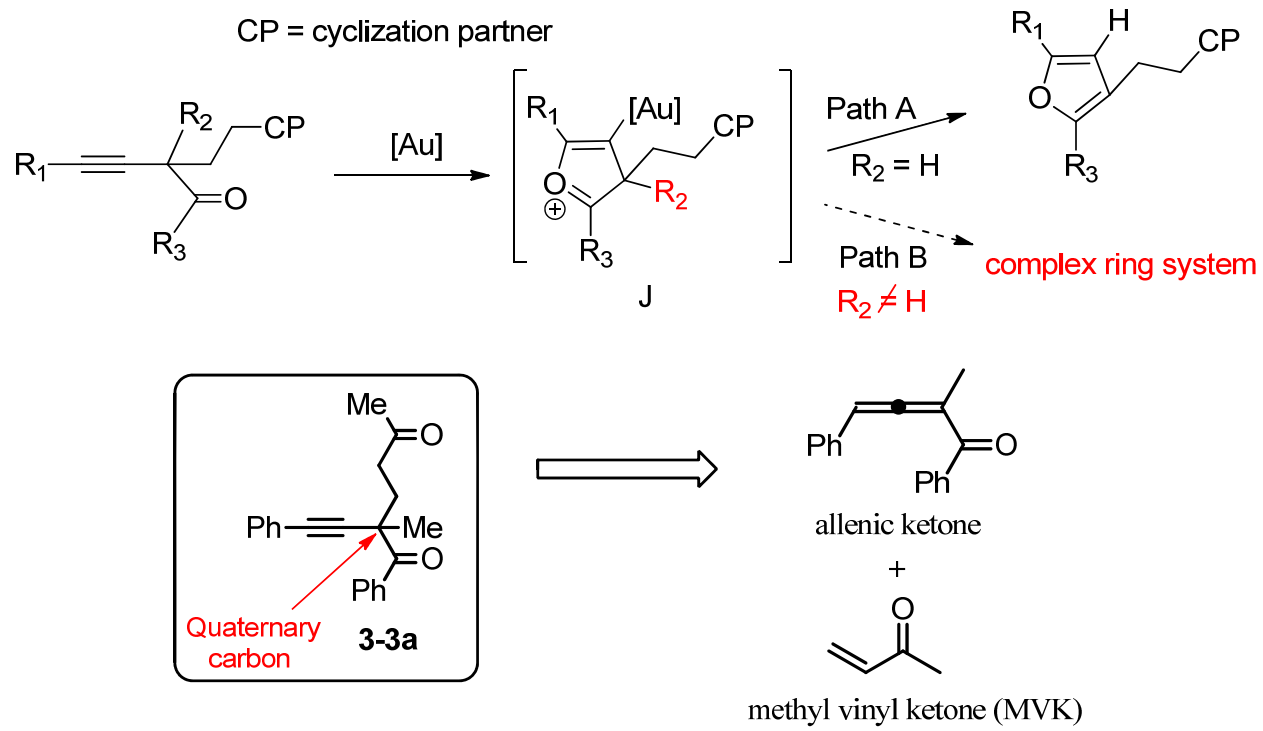

Scheme 12: Strategy for intramolecular-oxygen-transferred reactions. 

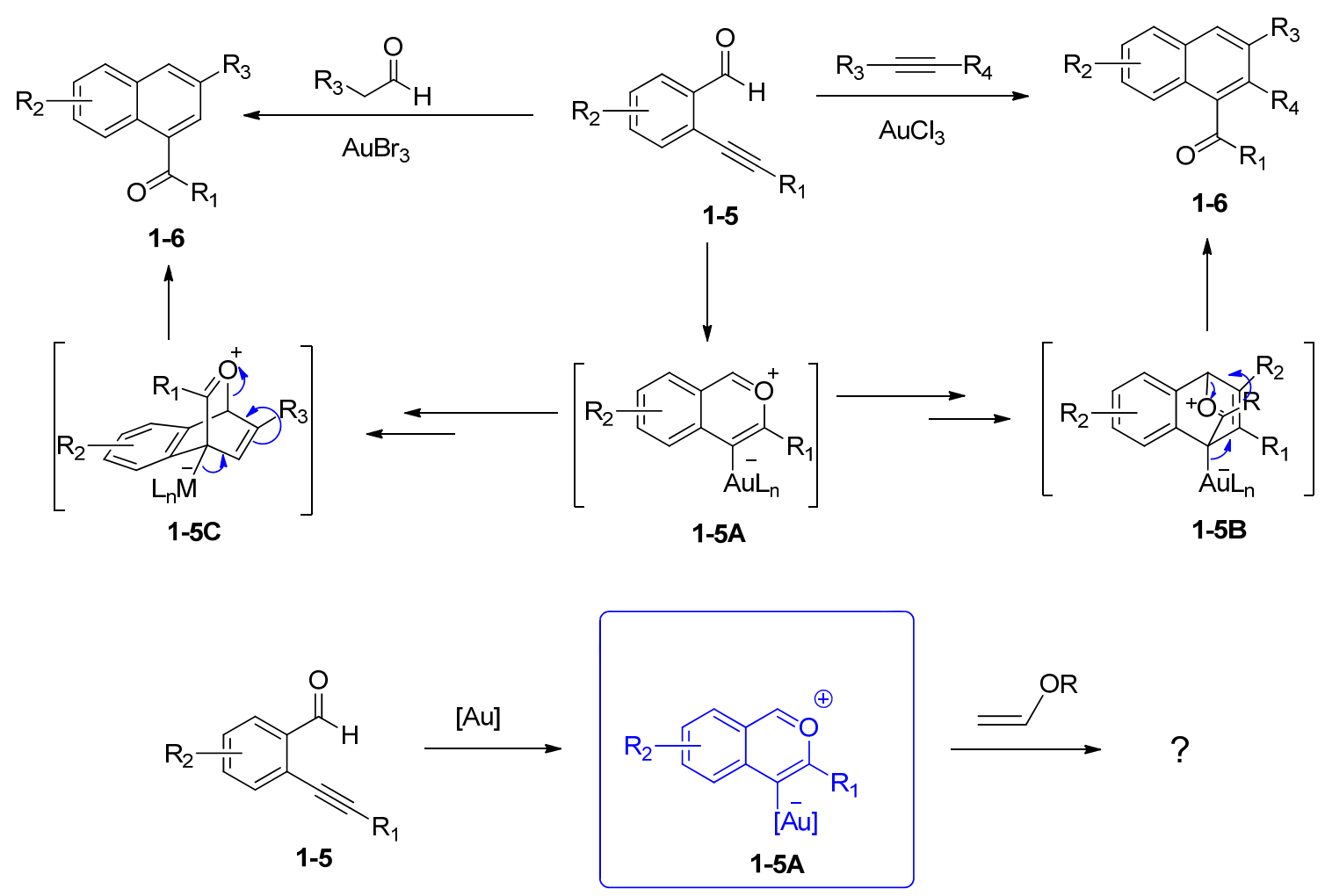

Scheme 13. Gold-catalyzed benzannulations and our aim.

Gold-catalyzed benzannulations has been well documented in the literature thanks to the work of Yamamoto and coworkers. ${ }^{22,36}$ In this reaction, 2-alkynyl benzaldehyde 1-5 reacts with an alkyne or aldehyde as cyclization partners to furnish naphthalene derivative 1-6 in an efficient manner (Scheme 13). From a mechanistic standpoint, the gold catalyst activated the triple bond of $\mathbf{1 - 5}$. This activation is followed by nucleophilic attack of carbonyl oxygen to generate oxonium intermediate 1-5A; its formation from a gold catalyst and 2-alkynyl benzaldehyde substrate is well-accepted. 1-5A underwent $[4+2]$ cycloaddition to generate intermediate $\mathbf{1 - 5 B}$, which subsequently rearranged to afford naphthalene susbstrate 1-6. However, in the presence of an aldehyde, intermediate 1-5A underwent reverse electron demand Diels Alder reaction with the enol form of aldehyde to generate intermediate $\mathbf{1 - 5 C}$, which further rearranged to furnish naphthalene derivative 1-6. We posited that in the presence of a suitable gold catalyst, 2-alkynyl 
benzaldehyde 1-5 would generate an oxonium intermediate, which could undergo reaction with a vinyl ether and yield synthetically interesting products; the details of the scope and mechanism of this reaction will be discussed in Chapter 4 (Scheme 13).

\subsection{Ligand design in gold catalysis}

In many palladium-catalyzed coupling reactions, such as the Suzuki reaction, the catalyst loading can be reduced to ppm levels ${ }^{37}$ but the common practice to use $5 \%$ loading in gold catalysis often makes it impractical to use in large scale synthesis. Ligands play a major role in the tuning of reactivity of gold catalysis and hence they hold the key to improving turnover numbers, but a rational understanding of ligand effects in gold catalysis is lacking, despite the intensive effort of notable researchers. Professor Dean Toste (UC Berkley) correctly articulated the crux of the limitations on ligand effects in gold catalysis in his recent Chemical Reviews paper (2008, 3351-3378). ${ }^{38}$ In his conclusions, the author states "although a great deal of empirical information is now available ... in homogeneous Au catalysis, the development of new catalysts and reactions continues to rely upon trial and error." Rational design of suitable ligands to achieve better efficiency is almost non-existent in gold catalysis and needs careful attention. Although the catalytic cycle of gold (Scheme 16) is well known, a rational design of ligands to improve its efficiency has not been reported yet. The influence of ligands on reactivity is not clear at all. For example: Toste and co-workers reported on the gold-catalyzed hydroamination of allenes ${ }^{39}$ 1-27 and ring expansion of propargyl cyclopropanols. ${ }^{40}$ They determined that in these two reactions, electron deficient phosphine ligands (e.g. $\left.\left(p-\mathrm{CF}_{3} \mathrm{C}_{6} \mathrm{H}_{4}\right)_{3} \mathrm{P}\right)$ accelerate the reaction whereas electron rich phosphine ligands slow down the reaction (Scheme 14a). However, in the majority of 
gold-catalyzed reactions, electron deficient phosphine ligands like $\left(p-\mathrm{CF}_{3} \mathrm{C}_{6} \mathrm{H}_{4}\right)_{3} \mathrm{P}$ don't work well; instead, electron rich ligands work better (Scheme 14b). ${ }^{41}$ These inconsistencies clearly underscore the complexity of gold catalysis. Moreover, the deactivation of gold is ubiquitous in gold catalysis, but how a ligand affects gold deactivation is unknown. The deactivation of gold catalyst further complicates the predictability of ligand performance in gold catalysis.

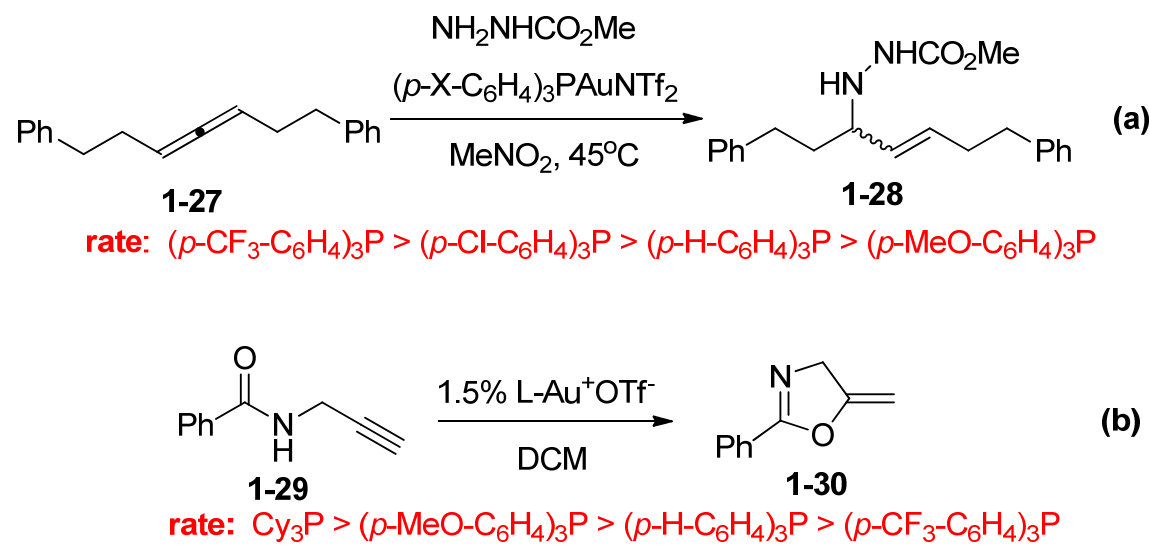

Scheme 14: Ligand effect in hydroamination of allenes 1-27 and cycloisomerization of propargyl amines 1-29.

There have been few reactions in the literature that achieved high turnover numbers (TONs) using gold catalysts. Notable examples include the $\left[(\mathrm{NHC}) \mathrm{Au}^{\mathrm{I}}\right]$-catalyzed hydration of alkyne, reported by Nolan and coworkers; ${ }^{42}$ the hydroamination of alkynes with a hyperhalogenated carba-closo-dodecaborate anionic ligand, reported by Lavallo and coworkers; ${ }^{43}$ and the ester assisted hydration of alkynes catalyzed by small gold clusters, reported by Corma and coworkers. ${ }^{44}$ In some exceptional cases, even higher turnovers can be achieved but at the cost of employing relatively high temperatures (e.g. $120^{\circ} \mathrm{C}$ ). More recently, in 2013, extremely high turnover numbers were achieved by Hashmi and coworkers ${ }^{45}$ in the intramolecular addition of diol to alkyne 1-31 using only $0.000001 \mathrm{~mol} \%$ of $\mathrm{N}$-Acyclic carbene (NAC)-based silsesquioxane-substituted 
mononuclear homogeneous gold catalyst, shown in Scheme 15. The high turnover numbers could be attributed to the fact that these bulky silsesquioxane substituted NAC based ligands have a stabilizing effect on the catalyst.
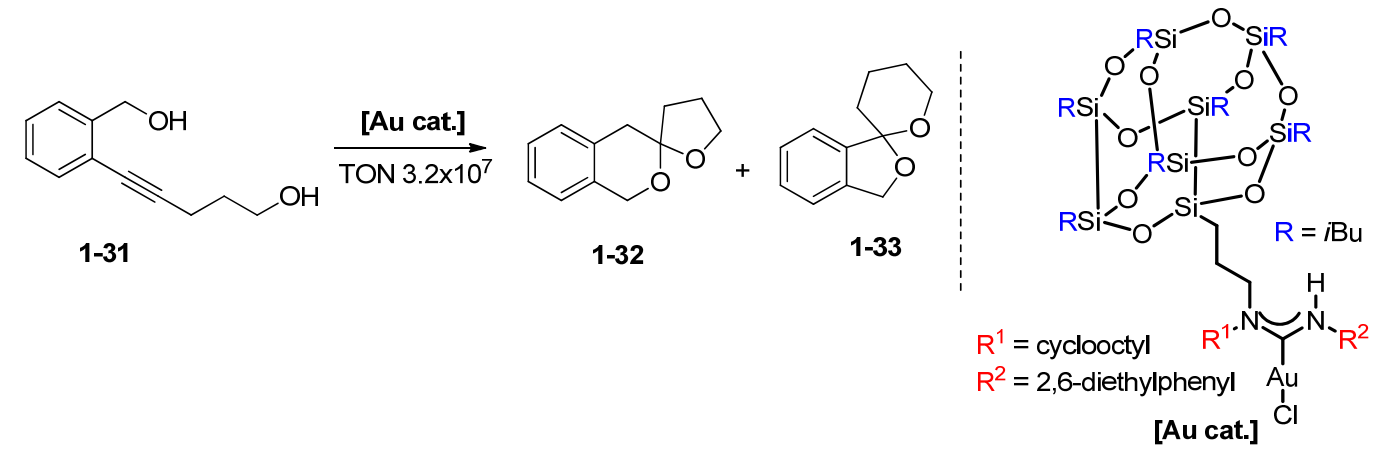

Scheme 15: Intramolecular addition of diol to alkyne with highly active mononuclear gold catalyst.

We became interested in developing a rational protocol to design ligands for goldcatalyzed reactions. To achieve this goal we carried a structure-activity relationship (SAR) study between the structure of a ligand and its reactivity. We focused our study on phosphine ligands since phosphine ligands are the most versatile neutral $2 \mathrm{e}^{-}$donor ligands available and their soft base character is compatible with gold, which is a soft Lewis acid. Moreover, ${ }^{31} \mathrm{P}$ NMR spectroscopy is a helpful and readily available tool in understanding the effect of ligands in transition metal catalysis. This tool allowed different phosphinebased ligands to be screened in our laboratory.

Typical gold catalytic cycle: It is well accepted that most gold-catalyzed reactions go through three major stages (Scheme 16). In stage 1 , a nucleophile attacks a $\left[\mathrm{AuL}_{n}\right]^{+}-$ activated alkyne (or alkene) A to form a trans-alkenyl gold complex intermediate $\mathbf{B}$ (or an alkyl gold complex in the case of alkenes). In stage 2 , the resulting vinyl complex reacts with an electrophile $\left(\mathrm{E}^{+}\right)$, usually a proton, to yield the final product via protodeauration, which also regenerates the cationic gold species (Scheme 16). Additionally, in almost all 
gold-catalyzed reactions, decay or deactivation of the gold catalyst takes place via the reduction of cationic gold to gold(0) (stage 3$)$.

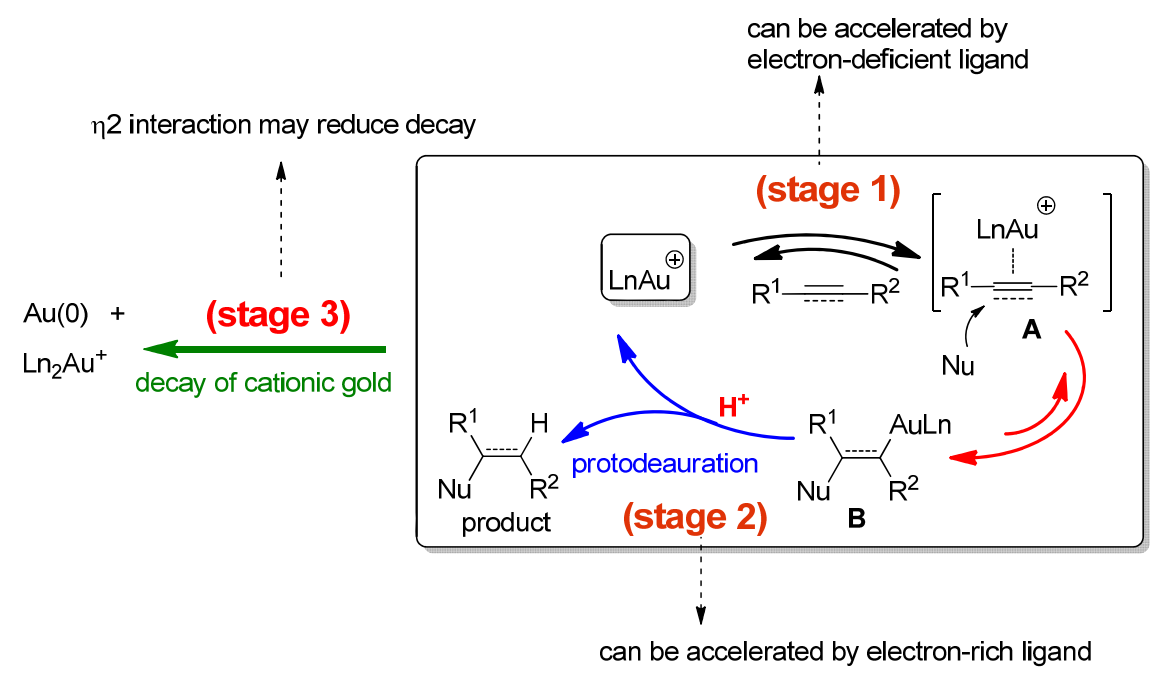

Scheme 16: Typical gold catalytic cycle.

From a previous paper from our group ${ }^{41}$ on ligand effects and ligand design on homogeneous gold catalysis, we found that two scenarios were possible in gold-catalyzed reactions: in the first one (type I) the nucleophilic addition to alkyne/allene/alkene (stage 1) is the rate-determining step. Therefore, electron-deficient ligands ought to speed-up this stage. ${ }^{41}$ In type I gold-catalyzed reaction, electron poor ligands give faster reaction rates. In type II, the regeneration of cationic gold catalyst (e.g., protodeauration) is the rate-determining step (stage 2). Therefore, electron-rich ligands ought to facilitate this stage. In type II gold-catalyzed reactions, electron rich ligands increase the reaction rate. These electronic effects hold true when the decay of cationic gold is not significant. With this background knowledge, we planned to develop a broadly applicable, readily prepared cationic gold catalyst that is efficient at low loading levels and mild reaction temperatures. 


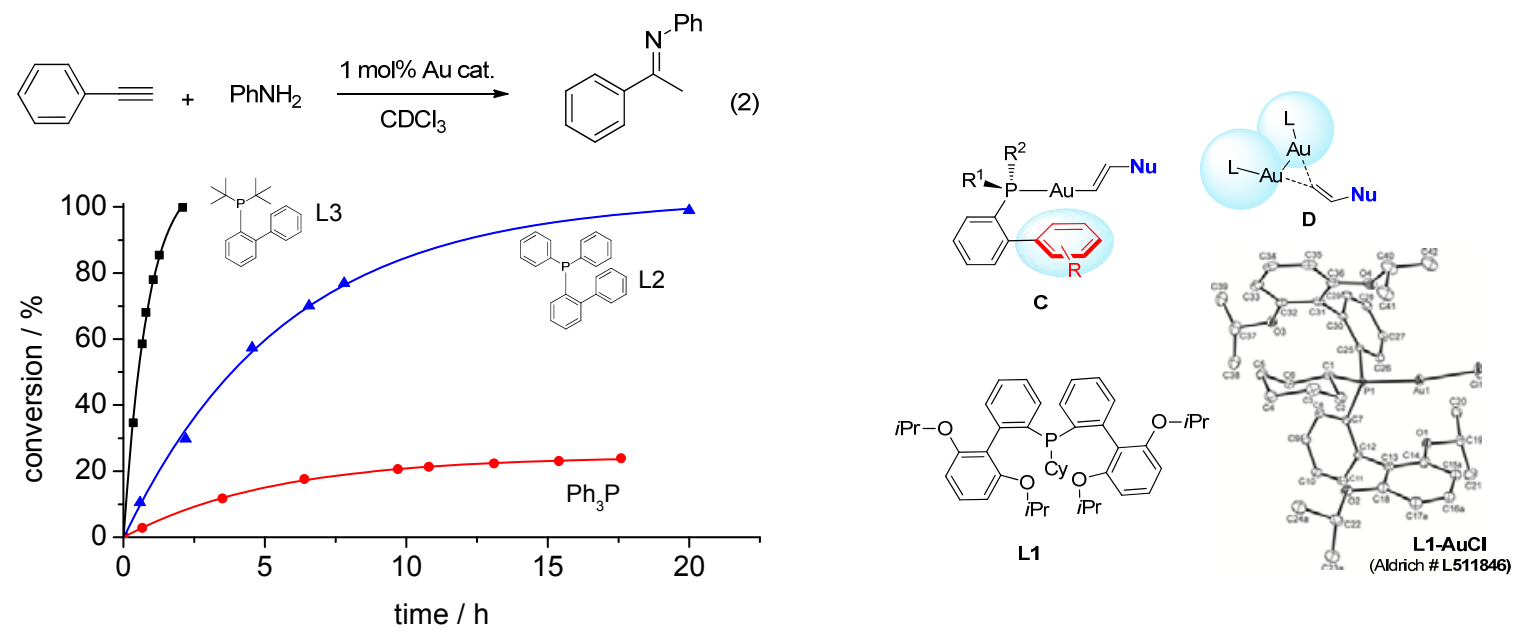

Figure 1: Electronic effect in hydroamination reaction and ligand L1.

To design such a gold catalyst we focused on the three major factors that account for the high loading in gold catalysis. These factors are: (i) the decay of the gold catalyst during the reaction, (ii) the formation of off-cycle gold species, and (iii) the mismatch of electronic effects within the gold ligand. We used the intermolecular hydroamination of phenyl acetylene with aniline as a model reaction for our study. As seen in Figure 1, a ligand like $\mathrm{Ph}_{3} \mathrm{P}$ is not a good choice since the reaction is relatively slow (i.e. $20 \%$ conversion after $18 \mathrm{~h}$ ). It was observed that the presence of a biphenyl group and introduction of two electron rich $t$-Bu groups (L3, JohnPhos) produced an even faster rate and full conversion to product. We hypothesized that two sterically demanding biaryls on the phosphine ligand could surround the gold center further and discourage the formation of D (Figure 1). Thus, we designed ligand L1 (featuring two electron-rich and sterically demanding ortho-biphenyl groups and one electron rich cyclohexyl group. We prepared L1 in a single step from commercially available starting materials. The crystallographic structure of L1-AuCl (Figure 1) demonstrated that the two ortho-biphenyl motifs were able to surround or embed the gold center. This L1-AuCl was found to be highly efficient 
at extremely low loadings in $\mathrm{C}-\mathrm{C}, \mathrm{C}-\mathrm{O}$ bond forming and cycloisomerization reactions at relatively low temperatures, the details of which will be described in Chapter 5 . 


\subsection{Outline of the dissertation:}

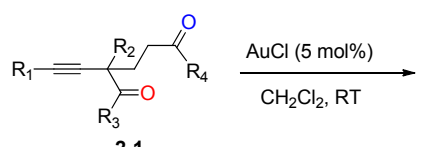

2-1

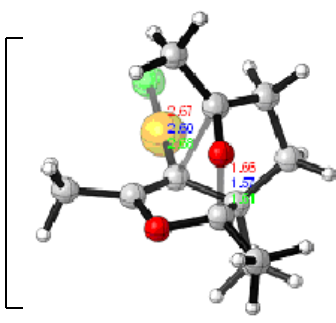

Chapter 1: Gold catalyzed intamolecular oxygen transfer reactions.

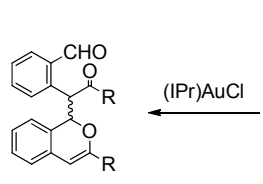

4-4

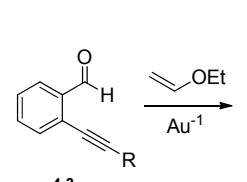

4-3

2-2
型

22 examples

up to $>99 \%$ yield
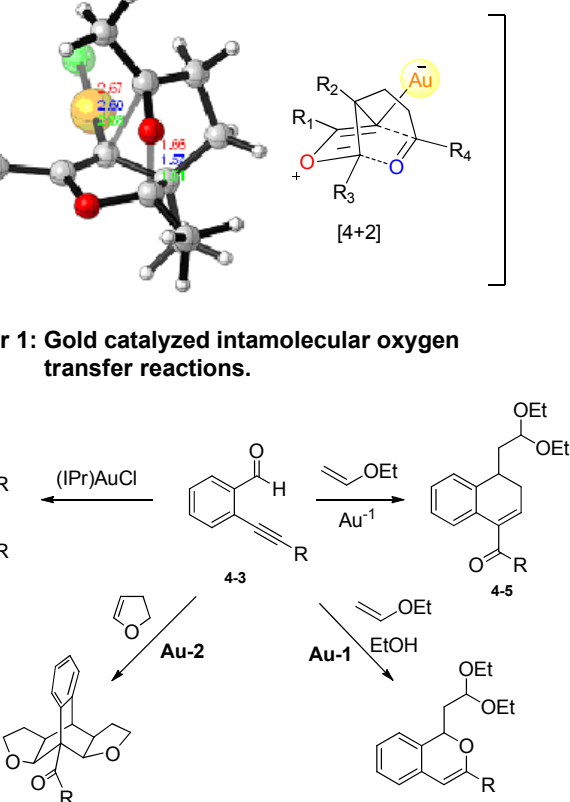

4-7

(IPr)AuCl

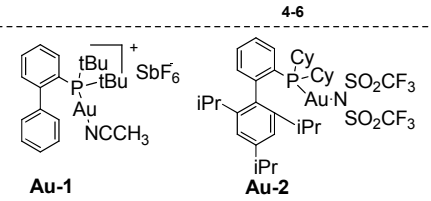

Chapter 3: Gold catalyzed cyclizations of 2-alkynyl benzaldehydes.

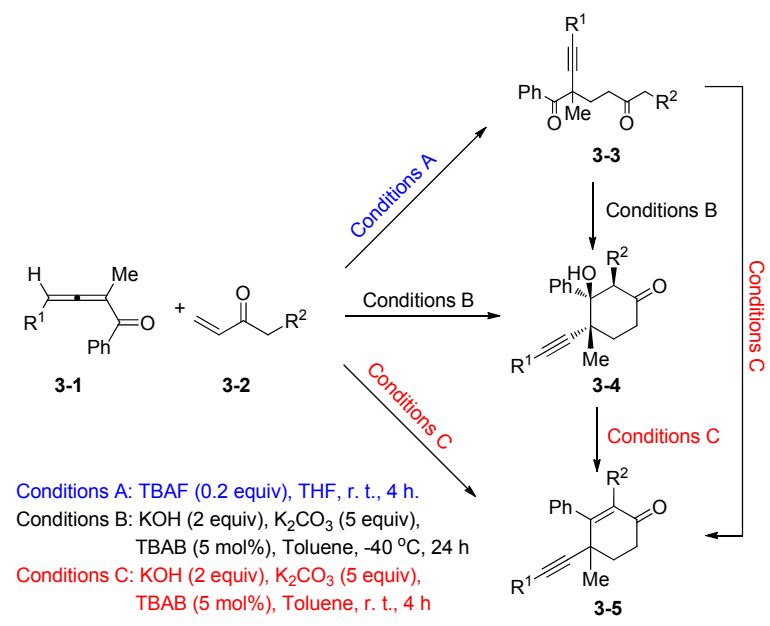

Chapter 2: Tandem Michael addition/aldol reaction of allenic ketones with vinyl ketones.

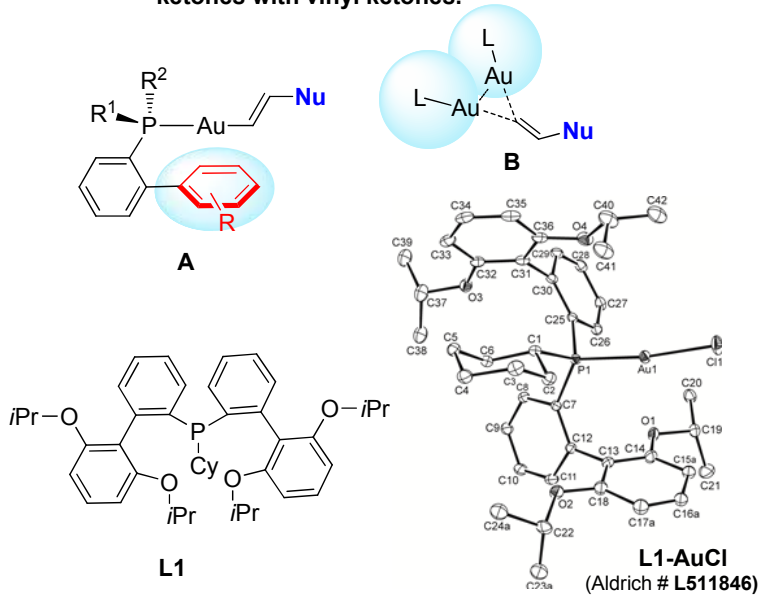

Chapter 4: Rational ligand design and broadly applicable ligand L1-AuCl. 


\section{CHAPTER 2. GOLD-CATALYZED INTRAMOLECULAR OXYGEN TRANSFER REACTIONS OF 2-ALKYNYL-1,5-DIKETONES OR 2-ALKYNYL-5-KETOESTERS}

\subsection{Background}

During the past few years our group has been working towards the development of efficient and selective gold-catalyzed transformations to discover simple approaches for the synthesis of interesting organic compounds. A major breakthrough occurred in 2008 while investigating the reactivity of allenoates to afford $\gamma$-lactones; our group discovered the formation of stable organogold(I) complexes. ${ }^{46}$ This important discovery supported the postulated mechanism of gold catalysis at the time, providing the first experimental evidence to support the mechanism of Au-catalyzed reactions of carbon-carbon multiple bonds, including alkynes/allenes/alkenes.

Continuing our efforts to explore gold-catalyzed transformations, we became interested in exploring the reactivity of 2-alkynyl-1,5-dicarbonyl for the construction of $\alpha, \beta$ unsaturated enones or similar compounds. This class of compounds has been at the forefront of synthetic organic chemistry since its inception, not only because these compounds are important building blocks in organic synthesis but also because the conjugated enone substructure in itself is a significant motif in natural products or 
biologically active compounds. ${ }^{47-53}$ Aldol condensations and Wittig-type reactions have been utilized for the construction of this moiety for decades. ${ }^{54-62}$ Recently though, an oxygen transfer from a carbonyl group to a carbon-carbon triple bond, also known as alkyne-carbonyl metathesis, has attracted the interest of synthetic chemists because this methodology could be an efficient, as well as atom-economical alternative to the Wittig reaction through the simultaneous formation of a new carbon-carbon double bond and carbonyl group. ${ }^{63-74}$ In this regard, many Lewis or Brønsted acid catalyzed intermolecular or intramolecular alkyne-carbonyl metathesis reactions have been extensively developed (Scheme 17, top). ${ }^{75-80}$ Notably, by using tethered alkynylketones as the substrates, Yamamoto and co-workers reported the gold- or TfOH-catalyzed oxygen-transfer reactions to give highly substituted cyclic enones as the products (Scheme 17, bottom). ${ }^{17,27,81}$

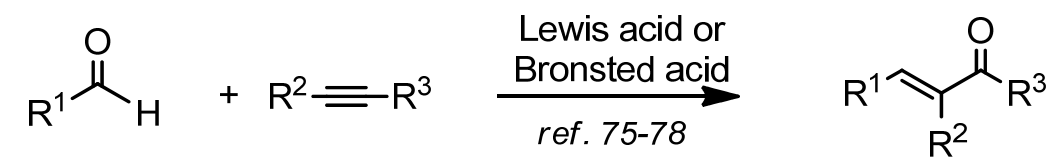

Lewis acid: $\operatorname{In}(\mathrm{OTf})_{3}, \mathrm{GaCl}_{3}, \mathrm{Yb}(\mathrm{OTf})_{3}$, $\mathrm{AgSbF}_{6}, \mathrm{BF}_{3}\left(\mathrm{OEt}_{2}\right), \mathrm{SbF}_{5}$

Bronsted acid: $\mathrm{HBF}_{4}$, TFA, TfOH
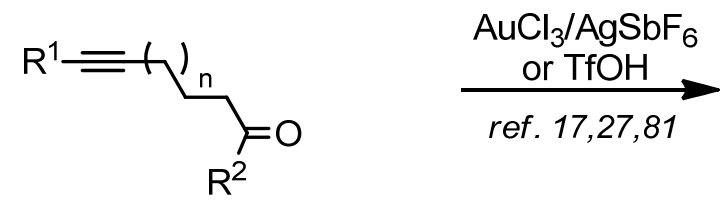<smiles>[R]C(=O)C1=C([R])CCC1</smiles>

Scheme 17. Lewis or brønsted acid- and gold-catalyzed intermolecular or intramolecular alkynecarbonyl metathesis.

A few years ago, we investigated the reactivity of alkynylenolates and other extended enolates ${ }^{82-85}$ and found that 2-alkynyl-1,5-dicarbonyl derivatives could be conveniently obtained from the tetra-n-butylammonium fluoride (TBAF) mediated Michael addition of activated allenes to electron-deficient olefins. The detailed synthesis will be discussed in 
Chapter $3 .^{35,86,87}$ Since two carbonyls and a carbon-carbon triple bond are installed in 2-1, we envisioned that an oxonium intermediate could arise from one of the carbonyls and the triple bond, and engender novel transformations. The chemistry of the oxonium ions formed from alkynylic aldehydes or ketones by mediation of transition metals, Lewis acids, Brønsted acids, or even electrophiles such as iodine, has attracted a lot of attention because these intermediates could undergo both inter- and intramolecular cycloadditions to carbon-carbon multiple bonds to give products of synthetic importance. ${ }^{22,36,81,88-123}$ Because of our continuous interest in gold catalysis, ${ }^{5,11,13,38,46,124-130}$ we subjected compound 2-1a to gold catalysis and found that the reaction, using $\mathrm{AuCl}$ as the catalyst, cleanly furnished cyclopentenyl ketone 2-2a, an intramolecular-oxygen-transferred product, in excellent yields after only $5 \mathrm{~min}$ at room temperature (Scheme 18). ${ }^{131}$

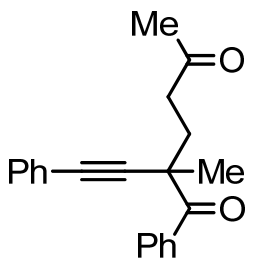

2-1a

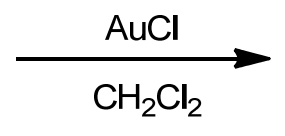

$\mathrm{CH}_{2} \mathrm{Cl}_{2}$

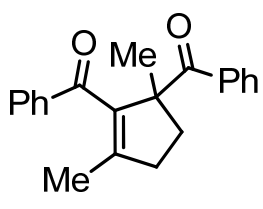

2-2a, $99 \%$

\section{Scheme 18: Gold-catalyzed oxygen transfer reaction.}

\subsection{Results and discussion}

Highly substituted five-membered rings bearing a quaternary carbon tethered to a carbonyl group are often found in natural products or biologically active compounds. Selected examples include Xestenone, ${ }^{132,133}$ Chloriolin-A, ${ }^{134}$ Spergulagenin- $\mathrm{A}^{135-138}$ and Saussureal ${ }^{139-141}$ (Scheme 19). Methods for the construction of these highly substituted carbocycles are rare in literature. Thus, we studied the scope of the gold-catalyzed intramolecular oxygen transfer reaction and developed a fast and efficient synthetic 
method to construct this important substructure. Various substrates were subjected to this reaction; the results are summarized in Table 1.

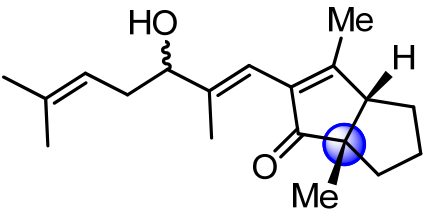

Xestenone

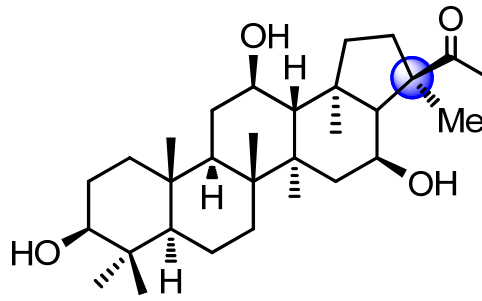

Spergulagenin-A

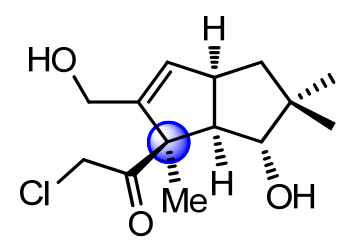

Chloriolin-A

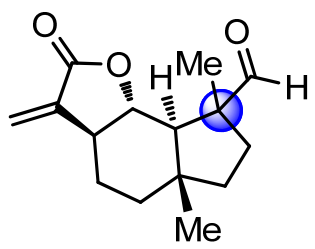

Saussureal

Scheme 19: Selected examples of natural products having a carbonyl tethered quaternary carbon on a five-membered ring.

It was found that the reactions of 2-alkynyl-1,5-diketones 2-1 with either aromatic or aliphatic groups substituted at the $\mathrm{R}_{1}$ position proceeded smoothly, and the corresponding products 2-2a-2-2i were isolated in excellent yields (Table 1, entries 1-9). The variation on $\mathrm{R}_{4}$ from methyl to ethyl and phenyl did not have a deleterious effect on the reaction, and the corresponding products $\mathbf{2 - 2} \mathbf{j}-\mathbf{2}-\mathbf{2} \mathbf{m}$ were obtained in very good yields (Table 1 , entries 10-13). Also, replacing the phenyl group at the $\mathrm{R}_{3}$ position with a methyl group did not diminish the efficiency of the reaction (Table 1, entry 14). To our surprise though, when the phenyl group at the $\mathrm{R}_{3}$ position was replaced by an ethoxy group, that is, when 2-alkynyl-5-ketoesters were used, the reaction still proceeded as smoothly and efficiently as before, although longer reaction times were required. The only exception occurred with 2-1u, from which $\mathbf{2 - 2} \mathbf{u}$ was isolated in only $50 \%$ yield, along with unidentified 
products (Table 1, entries 15-22). For the 2-alkynyl-5-ketoester substrate $\mathbf{2 - 1 w}$ bearing an aliphatic group at the $\mathrm{R}_{1}$ position, the reaction did not take place (Table 1, entry 23 ).

Table 1: Gold-catalyzed intramolecular oxygen transfer reactions of 2-alkynyl-1,5-diketones or 2alkynyl-5-ketoesters. ${ }^{[a]}$

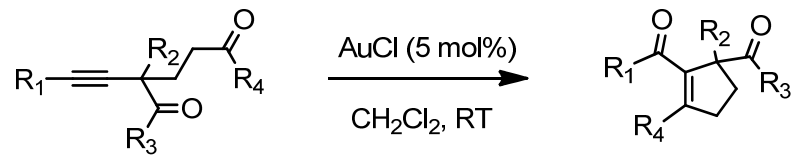

\begin{tabular}{|c|c|c|c|c|c|}
\hline entry & $\mathrm{R}_{1} / \mathrm{R}_{2} / \mathrm{R}_{3} / \mathrm{R}_{4}$ & yield/2-2 ${ }^{[b]}$ & entry & $\mathrm{R}_{1} / \mathrm{R}_{2} / \mathrm{R}_{3} / \mathrm{R}_{4}$ & yield/2-2 ${ }^{[b]}$ \\
\hline 1 & $\mathrm{Ph} / \mathrm{Me} / \mathrm{Ph} / \mathrm{Me} \mathrm{2-1a}$ & $2-2 a, 98$ & 13 & $p-\mathrm{ClC}_{6} \mathrm{H}_{4} / \mathrm{Me} / \mathrm{Ph} / \mathrm{Ph} \mathbf{2 - 1 m}$ & $2-2 m, 99$ \\
\hline 2 & $p-\mathrm{MeOC}_{6} \mathrm{H}_{4} / \mathrm{Me} / \mathrm{Ph} / \mathrm{Me}$ 2-1b & 2-2b, 99 & 14 & $\mathrm{Ph} / \mathrm{Me} / \mathrm{Me} / \mathrm{Me}$ 2-1n & 2-2n, 92 \\
\hline 3 & $p-\mathrm{ClC}_{6} \mathrm{H}_{4} / \mathrm{Me} / \mathrm{Ph} / \mathrm{Me} 2-1 \mathrm{c}$ & 2-2c, 92 & 15 & $\mathrm{Ph} / \mathrm{Me} / \mathrm{OEt} / \mathrm{Me}$ 2-10 & 2-2o, 88 \\
\hline 4 & $n-\mathrm{C}_{6} \mathrm{H}_{13} / \mathrm{Me} / \mathrm{Ph} / \mathrm{Me}$ 2-1d & 2-2d, 99 & 16 & $p-\mathrm{MeOC}_{6} \mathrm{H}_{4} / \mathrm{Me} / \mathrm{OEt} / \mathrm{Me} 2-1 \mathrm{p}$ & 2-2p, 92 \\
\hline 5 & $i-\mathrm{Pr} / \mathrm{Me} / \mathrm{Ph} / \mathrm{Me}$ 2-1e & $2-2 e, 96$ & 17 & $p-\mathrm{ClC}_{6} \mathrm{H}_{4} / \mathrm{Me} / \mathrm{OEt} / \mathrm{Me}$ 2-1q & $2-2 q, 90$ \\
\hline 6 & $t$-Bu/Me/Ph/Me 2-1f & 2-2f, 95 & 18 & $\mathrm{Ph} / \mathrm{Me} / \mathrm{OEt} / \mathrm{Ph}$ 2-1r & 2-2r, 91 \\
\hline 7 & $\mathrm{Me} / \mathrm{Me} / \mathrm{Ph} / \mathrm{Me}$ 2-1g & $\mathbf{2 - 2 g}, 91$ & 19 & $p-\mathrm{MeOC}_{6} \mathrm{H}_{4} / \mathrm{Me} / \mathrm{OEt} / \mathrm{Ph}$ 2-1s & $2-2 s, 92$ \\
\hline 8 & $\mathrm{Bn} / \mathrm{Me} / \mathrm{Ph} / \mathrm{Me}$ 2-1h & 2-2h, 94 & 20 & $p-\mathrm{ClC}_{6} \mathrm{H}_{4} / \mathrm{Me} / \mathrm{OEt} / \mathrm{Ph}$ 2-1t & $2-2 t, 92$ \\
\hline 9 & $\mathrm{CypCH}_{2} / \mathrm{Me} / \mathrm{Ph} / \mathrm{Me}$ 2-1i & 2-2i, 99 & 21 & $\mathrm{Ph} / \mathrm{Bn} / \mathrm{OEt} / \mathrm{Me} \mathbf{2 - 1 \mathrm { u }}$ & $2-2 u, 50$ \\
\hline 10 & $\mathrm{Ph} / \mathrm{Me} / \mathrm{Ph} / \mathrm{Et}$ 2-1j & 2-2j, 98 & 22 & $\mathrm{Ph} / \mathrm{Bn} / \mathrm{OEt} / \mathrm{Ph}$ 2-1v & $2-2 v, 90$ \\
\hline 11 & $\mathrm{Ph} / \mathrm{Me} / \mathrm{Ph} / \mathrm{Ph}$ 2-1k & 2-2k, 93 & 23 & $n-\mathrm{C}_{6} \mathrm{H}_{13} / \mathrm{Me} / \mathrm{OEt} / \mathrm{Me} \mathbf{2 - 1 w}$ & NR \\
\hline 12 & $p-\mathrm{MeOC}_{6} \mathrm{H}_{4} / \mathrm{Me} / \mathrm{Ph} / \mathrm{Ph}$ 2-1I & 2-2l, 91 & $24^{[\mathrm{c}]}$ & $n-\mathrm{C}_{6} \mathrm{H}_{13} / \mathrm{Me} / \mathrm{OEt} / \mathrm{Me} \mathbf{2 - 1 w}$ & Complex \\
\hline
\end{tabular}

[a] General Reaction Conditions: 2-Alkynyl-1,5-Diketone or 2-Alkynyl-5-ketoester 2-1 (0.3 mmol), $\mathrm{CH}_{2} \mathrm{Cl}_{2}(2.0 \mathrm{~mL})$. For Alkynyldiketones, Reaction Time $=5$ minutes; For Alkynylketoesters, Reaction Time = 30 minutes; [b] Isolated Yields; [c] Reaction was conducted in toluene at $80{ }^{\circ} \mathrm{C}$. Cyp = Cyclopentanyl; NR = No Reaction.

The traditional mechanism of the oxygen-transfer reaction invoked a [2+2] pathway (Scheme 20, top) for the inter-/intramolecular alkyne-carbonyl metathesis. ${ }^{17,27,75-80,142}$ However, the fact that the oxygen-transfer reaction of 2-alkynyl-1,5-diketones or 2alkynyl-5-ketoesters could be completed in minutes at room temperature, and with higher yields than previously reported oxygen transfer prompted us to propose an alternative [4+2] mechanism (Scheme 20, bottom). We envisioned that a five-membered ring furanium $\mathbf{C}^{8,20,143-152}$ would be much easier to form than the seven-membered ring oxonium $\mathbf{A}$, this action is followed by a [4+2] cycloaddition of the furanium intermediate 
to the other carbonyl group to form the intermediate $\mathbf{D}$, which finally yields the product after several electron transfer steps.

We designed an isotopic labeling experiment to elucidate if the well-accepted [2+2] mechanism, or our newly proposed [4+2] pathway was responsible for the gold-catalyzed intramolecular oxygen transfer of 2-alkynyl-1,5-diketones and 2-alkynyl-5-ketoesters (Scheme 20). We speculated that if an ${ }^{18} \mathrm{O}$ atom could be incorporated into one of the carbonyls groups in the substrates, then the ${ }^{13} \mathrm{C}$ NMR of the reaction product could be used to locate the ${ }^{18} \mathrm{O}$ atom, ${ }^{153-155}$ and provide clues as to the more favorable mechanistic pathway. Hence, using alkynyldiketone $\mathbf{2 - 1} \mathbf{1 b}-{ }^{\mathbf{1}} \mathrm{O}\left(\mathrm{R}_{1}=p\right.$-methoxyphenyl, $\mathrm{R}_{2}=$ phenyl $)$ and alkynylketoester $\mathbf{2 - 1 0}-{ }^{18} \mathrm{O}\left(\mathrm{R}_{1}=\right.$ phenyl, $\mathrm{R}_{2}=$ ethoxy $)$ as the model substrates, we surmised that if the reaction followed a [2+2] route then the ${ }^{18} \mathrm{O}$ would end up on the left carbonyl group in $\mathbf{2 - 2 - { } ^ { 1 8 }} \mathbf{O}-\mathbf{a}$ (Scheme 20 , top), whereas it would be incorporated on the benzoyl group or ester group in $\mathbf{2 - 2 - { } ^ { 1 8 }} \mathbf{O}$-b if the reaction followed a [4+2] pathway (Scheme 20, bottom).

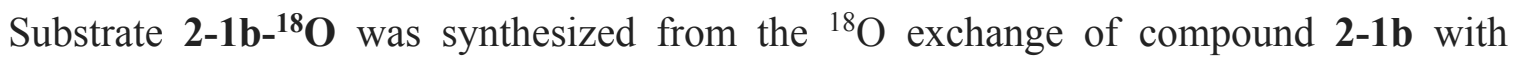
$\mathrm{H}_{2}{ }^{18} \mathrm{O}$ under acidic conditions (Scheme 21). ${ }^{156}$ The ${ }^{18} \mathrm{O}$ exchange occurred only on the methyl carbonyl group, as indicated by ${ }^{13} \mathrm{C}$ NMR spectroscopy. A $0.05 \mathrm{ppm}(5 \mathrm{~Hz})$ upfield chemical shift ${ }^{153-155}$ was found on carbon 1 in the substrate, whereas no chemical shift change occurred on carbon 2 . The $\mathbf{2 - 1} \mathbf{b}-{ }^{18} \mathbf{O}$ substrate was subjected to the goldcatalyzed oxygen transfer reaction and the product $\mathbf{2 - 2 b}-{ }^{18} \mathbf{O}$ was obtained in quantitative yield, without any ${ }^{18} \mathrm{O}$ loss, as determined by its ESI mass spectrum. It was found that only carbon 4 in the product $\mathbf{2 - 2} \mathbf{b}-{ }^{18} \mathbf{O}$ exhibited the $0.05 \mathrm{ppm}(5 \mathrm{~Hz})$ upfield chemical shift in its ${ }^{13} \mathrm{C}$ NMR spectrum (Scheme 22). 


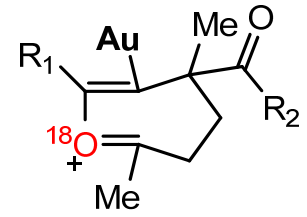<smiles>[R7]C#CC(C)(CC(C)=O)C([R2])=O</smiles>

$2-1-18 O$
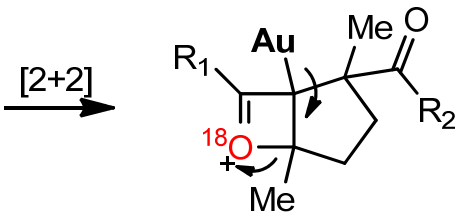

B<smiles>[B][10B]=C</smiles>

Au

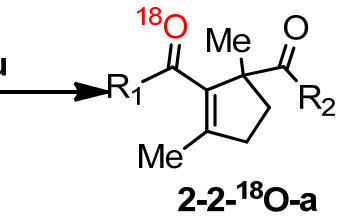

or<smiles>[R]C(=O)C1=C(C)CCC1([Y10])C([R])=O</smiles>

1b: $\mathrm{R}^{1}=p-\mathrm{MeOC}_{6} \mathrm{H}_{4}, \mathrm{R}^{2}=\mathrm{Ph}$

$\checkmark$ 10: $R^{1}=P h, R^{2}=O E t$

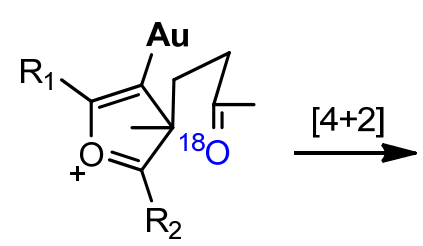

C

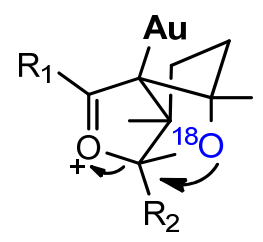

D

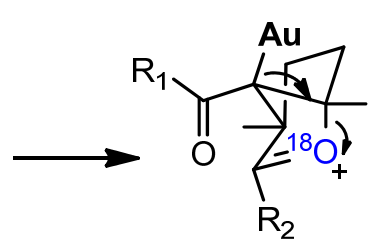

E

Scheme 20: ${ }^{18} \mathrm{O}$ isotopic labeling experiment for mechanistic studies.

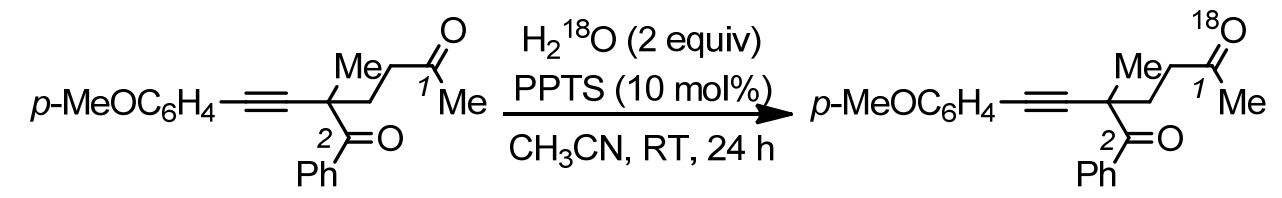

2-1b

$$
\text { 2-1b-18 } \mathrm{O}
$$

$38 \%{ }^{18} \mathrm{O}^{a}$
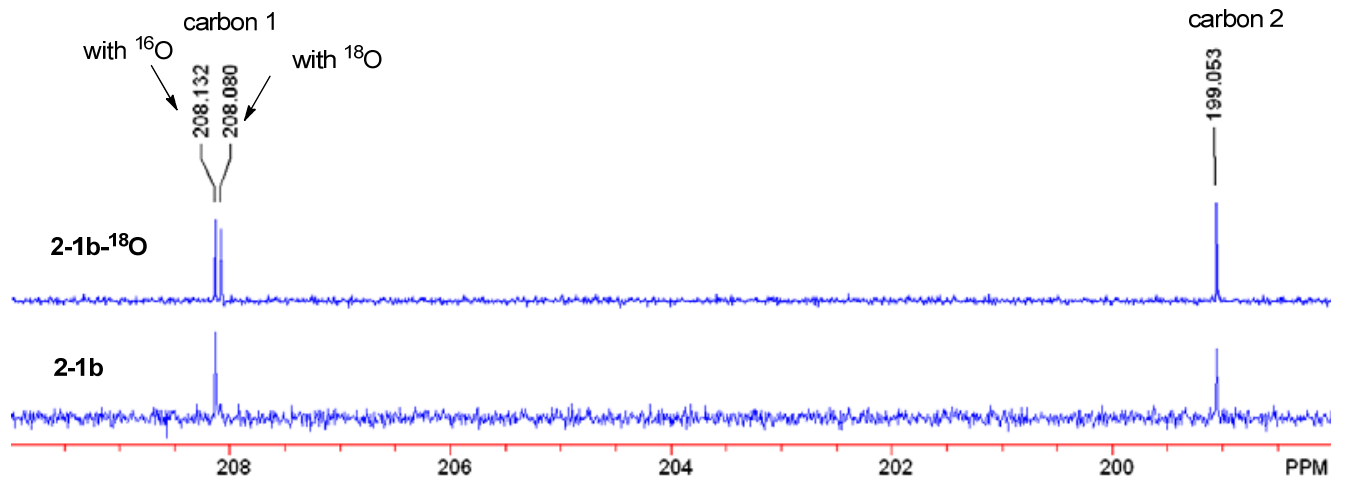

${ }^{a}$ Determined by MS (ESI).

Scheme 21: Synthesis of substrate $2-1 b-{ }^{18} \mathrm{O}$ and its ${ }^{13} \mathrm{C}$ NMR spectrum. 


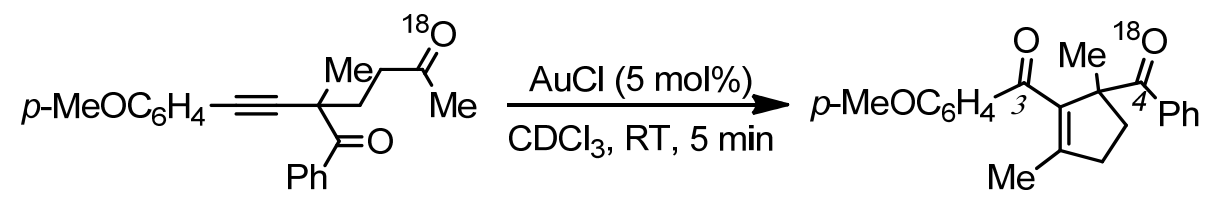

$2-1 b-{ }^{18} \mathrm{O}$

$38 \%{ }^{18} \mathrm{O}^{\mathrm{a}}$

carbon 4
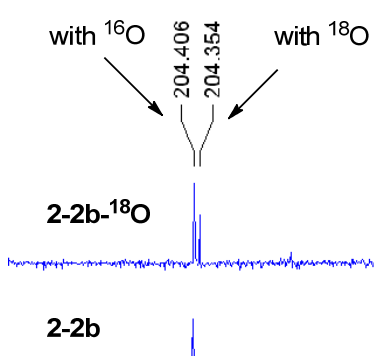

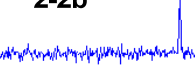

$\mathbf{2 - 2 b - 1 8} \mathbf{O},>99 \%{ }^{b}$

$38 \%{ }^{18} \mathrm{O}^{a}$

carbon 3

${ }^{a}$ Determined by MS (ESI). ${ }^{b}$ Determined by ${ }^{1} \mathrm{H}$ NMR.

Scheme 22: ${ }^{18} \mathrm{O}$ isotopic experiment of alkynyldiketone and its ${ }^{13} \mathrm{C}$ NMR spectrum.

By utilizing the same prior methodology, substrate $\mathbf{2 - 1 0}-{ }^{\mathbf{1 8}} \mathrm{O}$ was also synthesized from the ${ }^{18} \mathrm{O}$ exchange of compound 2-10 with $\mathrm{H}_{2}{ }^{18} \mathrm{O}$ under acidic conditions. Again, the ${ }^{18} \mathrm{O}$ exchange happened only at the methyl carbonyl group, as indicated by ${ }^{13} \mathrm{C} \mathrm{NMR}$ spectroscopy. A $0.05 \mathrm{ppm}(5 \mathrm{~Hz})$ upfield chemical shift was found only on carbon 1 (Scheme 23). Substrate $\mathbf{2 - 1 0 -}{ }^{\mathbf{1 8}} \mathbf{O}$ was subjected to the gold-catalyzed oxygen transfer reaction under the same conditions, and the corresponding product $\mathbf{2 - 2 0 -} \mathbf{- 1 8}^{\mathbf{O}} \mathbf{O}$ was isolated in good yield. By running its ${ }^{13} \mathrm{C}$ NMR spectrum, it was found that only carbon 4 at the ester group in the product $\mathbf{2 - 2 0 -}{ }^{\mathbf{1 8}} \mathbf{O}$ showed the $0.037 \mathrm{ppm}(3.7 \mathrm{~Hz})$ upfield chemical shift (Scheme 24). The absence of any detectable ${ }^{18} \mathrm{O}$ incorporation at carbon 3 in both products $\left(\mathbf{2 - 2 b -}{ }^{\mathbf{1 8}} \mathbf{O}\right.$ and $\left.\mathbf{2 - 2 0 -}{ }^{18} \mathbf{O}\right)$ demonstrated that the [2+2] pathway is disfavored, and instead it is the newly proposed [4+2] pathway that accounted for the mechanism of the gold-catalyzed intramolecular oxygen transfer of 2-alkynyl-1,5-diketones and 2-alkynyl5-ketoesters. 


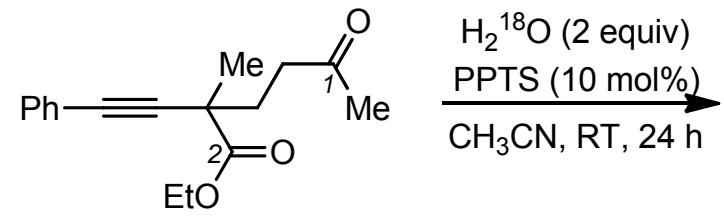

2-10

carbon 1

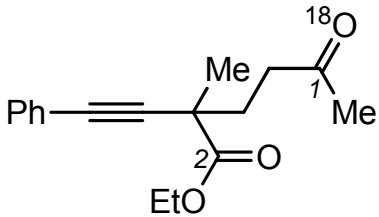

$2-10-{ }^{18} O$

carbon 2
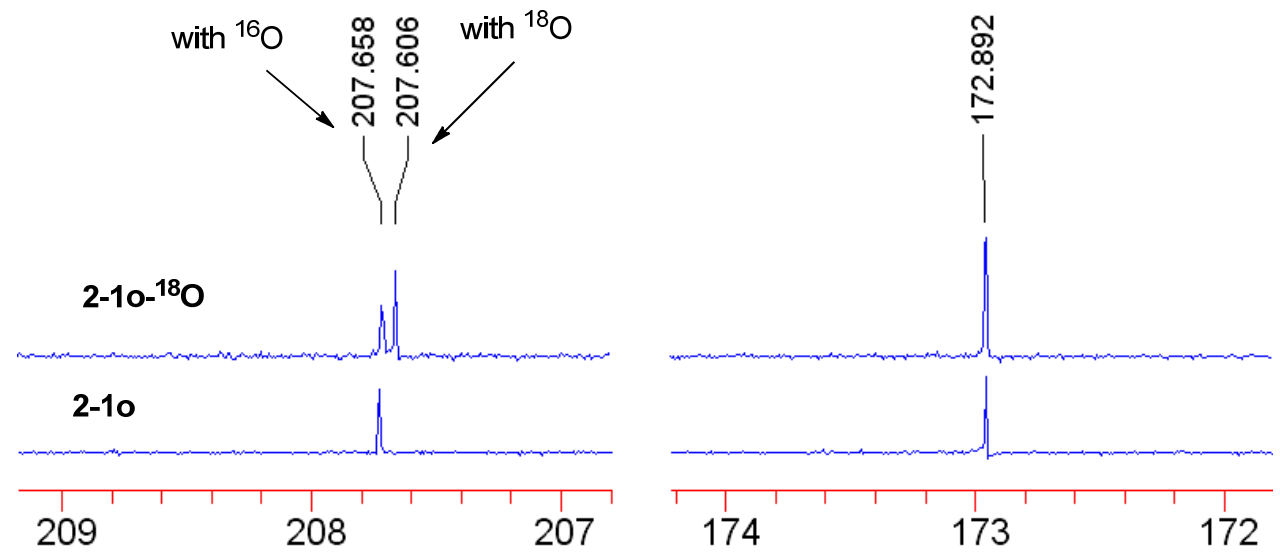

Scheme 23: Synthesis of substrate $2-10-{ }^{18} \mathrm{O}$ and its ${ }^{13} \mathrm{C}$ NMR spectrum.<smiles>CCOC(=O)C(C)(C#Cc1ccccc1)CCC(C)=O</smiles>

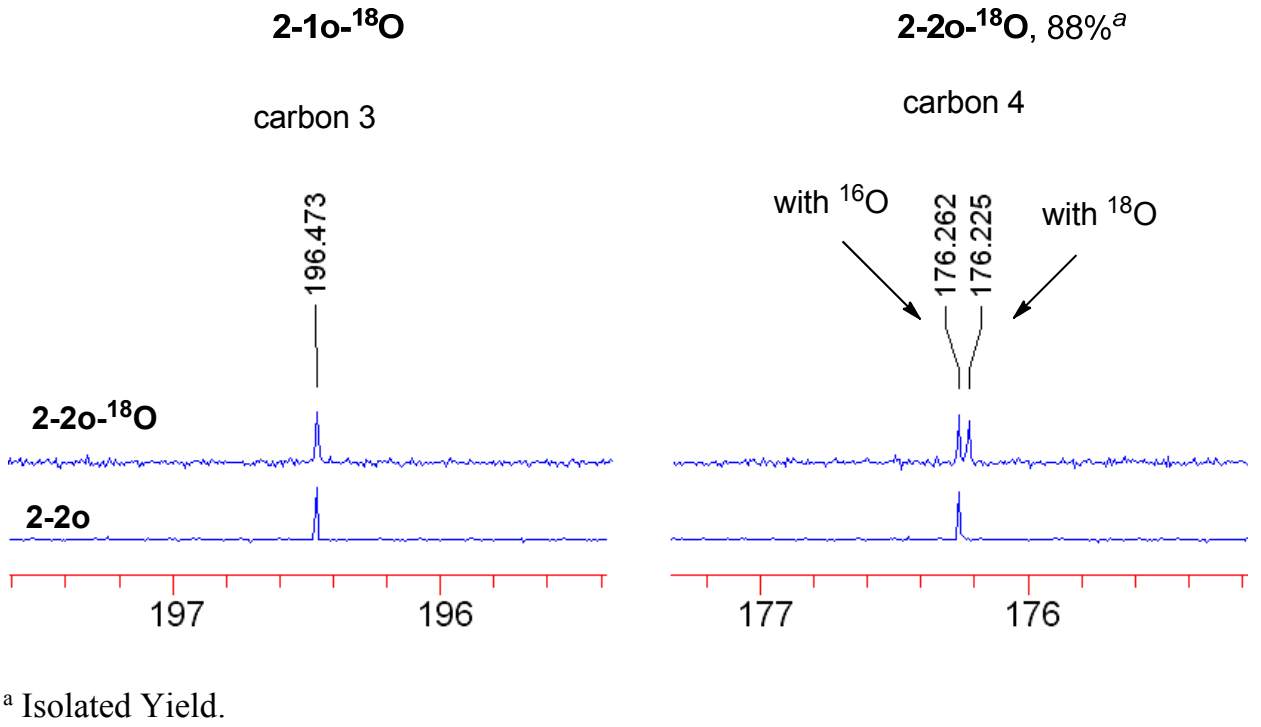

Scheme 24: ${ }^{18} \mathrm{O}$ isotopic experiment of alkynylketoester and its ${ }^{13} \mathrm{C}$ NMR spectrum. 
Alongside our experimental studies, we turned to quantum chemical calculations to seek further verification that the proposed [4+2] mechanism was the preferred pathway and to understand the origins of this selectivity. We also sought to clarify the mechanism of the competing [2+2] pathway, which had been invoked on a number of occasions to rationalize carbonyl-alkyne metathesis reactions. All calculations were performed with Gaussian 09. ${ }^{157}$ Stationary points were fully optimized with the B3LYP ${ }^{158-161}$ (hybrid GGA) and M06-2X $\mathrm{X}^{162}$ (hybrid meta-GGA) density functionals, using a fine grid for numerical integration. Both functionals have been utilized extensively in computational studies of $\mathrm{Au}(\mathrm{I})$-catalysis, ${ }^{25,163-169}$ although the M06-2X functional may be expected to describe non-bonding interactions with greater accuracy. For optimizations we used a combination of the Pople 6-31G(d) basis set for $\mathrm{C}, \mathrm{H}, \mathrm{O}$ and $\mathrm{Cl}$ and the LANL2DZ (HayWadt) basis including an effective core potential for $\mathrm{Au} .{ }^{170}$ Single point calculations were performed on these optimized structures with a larger (triple- $\zeta$ ) $6-311+\mathrm{G}(\mathrm{d}, \mathrm{p})$ basis set on $\mathrm{C}, \mathrm{H}, \mathrm{O}$ and $\mathrm{Cl}$. Where it was computationally tractable, we also performed optimizations at the "double-hybrid" density functional level of theory with the B2PLYP functional. ${ }^{171}$ This method replaces a fraction of the semi-local correlation energy by a non-local correlation energy expression that employs the Kohn-Sham orbitals in second order perturbation theory and delivers improved energetics over hybrid density functionals such as B3LYP. Geometries and energetics obtained at this level and from single point energy calculations at the SCS-MP2 level ${ }^{172}$ were also compared with the B3LYP and M06-2X results, to verify that the hybrid-GGA functionals gave geometries and energetics that agreed with these more demanding methods. Transition structures were confirmed by the presence of an imaginary harmonic vibrational frequency corresponding to a 
displacement along the proposed reaction coordinate. NBO version 3.1 was used for the calculation of Wiberg bond order (BO) from the natural bond orbitals. ${ }^{173}$

Competing $[2+2]$ and $[4+2]$ reaction coordinates were computed for a model substrate for which $R_{1}, R_{2}, R_{3}$ and $R_{4}$ are all methyl groups, allowing us to compare the performance of DFT at the hybrid-GGA level with more demanding B2PLYP and SCS-MP2 calculations. Experimentally, we demonstrated that similar substrates (Table 1, entry 7: $\mathrm{R}_{1}, \mathrm{R}_{2}, \mathrm{R}_{4}=\mathrm{Me} / \mathrm{R}_{3}=\mathrm{Ph}$ and entry $14: \mathrm{R}_{2}, \mathrm{R}_{3}, \mathrm{R}_{4}=\mathrm{Me} / \mathrm{R}_{1}=\mathrm{Ph}$ ) rearranged in $91-92 \%$ yield. We begin with a discussion of our newly proposed [4+2] pathway, for which the computed B2PLYP reaction coordinate is shown in Figure 2. The rate-limiting step in the rearrangement is the intramolecular nucleophilic addition to the Au-coordinated alkyne the activation barrier for this step is computed to be a modest $8.8 \mathrm{kcal} / \mathrm{mol}$, forming fivemembered ring oxonium intermediate $\mathbf{B}$ (via 5-endo-dig TS-1). Once this cyclization has occurred to form $\mathbf{C}$ the remaining transformations that lead to the rearranged product are all computed to be relatively facile. TS-2, formally a [4+2] cycloaddition, involving the formation of two new C-C and C-O $\sigma$-bonds, lies only $4.4 \mathrm{kcal} / \mathrm{mol}$ above the starting complex 2-1-Au. The barriers for TS-3, the opening of acetal intermediate C, and TS-4, the opening of oxonium $\mathbf{D}$, are very small indeed. At room temperature this process would be expected to occur readily, consistent with the 5 min reaction times observed experimentally.

B2PYLP optimized transition structures (TSs) along the [4+2] pathway are also shown in Figure 2. Rate-limiting TS-1 involves a 5-endo-dig cyclization, which is allowed according to Baldwin's rules. Bond formation in TS-5 can be seen to be highly asynchronous, with the forming C-O bond at $1.66 \AA$ (Wiberg BO 0.60) and the C-C bond 
at $2.67 \AA$ (Wiberg BO 0.12), however, this process is concerted as no intermediate exists between B and C. All of the computational levels examined suggest the rate-limiting step in the [4+2] pathway is carbonyl-oxygen addition to the Au-coordinated alkyne, giving energetic profiles similar to that shown in Figure 2 and optimizations with either B2PLYP, B3LYP or M06-2X density functionals result in similar geometries. The computed B3LYP energetic profile was also computed for experimental substrate 2-1a to compare with our results for the model system used. These calculations were in good accord with those shown in Figure 2, again with the 5-endo addition as rate limiting step.
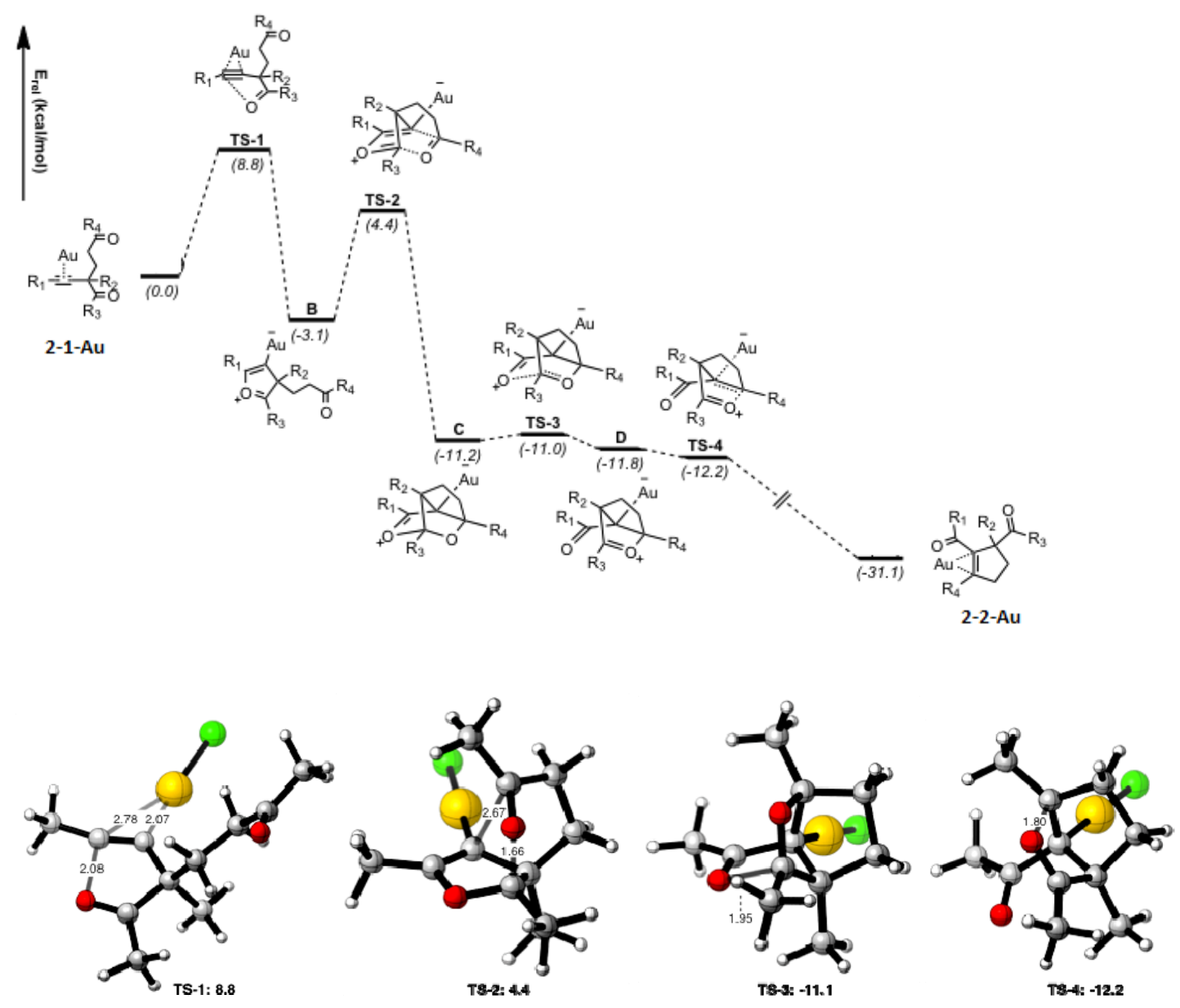

Figure 2: Above: B2PLYP/6-311+G(d,p)//B2PLYP/6-31G(d) (using LANL2DZ ECP on Au) Relative energy Profile computed for Both $[2+2]$ and $[4+2]$ pathways for a model substrate. $R_{1}, R_{2}, R_{3}, R_{4}=M e$, $\mathrm{Au}=$ AuCl. Below: B2PLYP/6-311+G(d,p)/B2PLYP/6-31G(d) computed stationary points for the model sytem. Energies relative to reactant 2-1-Au. Bond forming/breaking distances in $\AA$. 
Experimentally, we observed that alkynylketoesters underwent Au-catalyzed rearrangement more slowly than the alkynyldiketones. The computed [4+2] pathway for the rearrangement of a model alkynylketoester suggested that the overall energy change of reaction $(-31.7 \mathrm{kcal} / \mathrm{mol})$ is very close to that of the alkynyldiketone $(-31.1 \mathrm{kcal} / \mathrm{mol})$. However, the energetic barrier for 5 -endo cyclization (i.e. TS-1') is $13.4 \mathrm{kcal} / \mathrm{mol}$ (c.f. a value of $8.8 \mathrm{kcal} / \mathrm{mol}$ for the diketone) suggests the initial alkyne addition is more difficult for the ester substrate. Interestingly the relative energy of TS-2' at $19.2 \mathrm{kcal} / \mathrm{mol}$ means that the $[4+2]$ addition step is now more difficult than the cyclization step for the alkynylketoester. These values are consistent with the observations of the ester substrates reacting more slowly, although our proposed mechanism is still computed to be viable at room temperature. The transition structures for the rearrangement of the ketoester substrate are shown below in Figure 3.
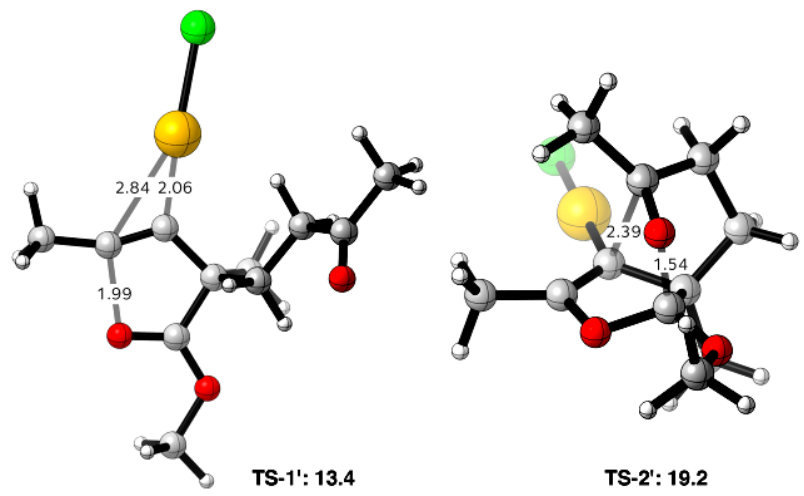

Figure 3: Proposed mechanism and B2PLYP/6-311+G(d,p)/B2PLYP/6-31G(d) computed stationary points for the oxetene pathway. Energies relative to reactant 2-1. Bond breaking distance in $\AA$.

We considered a number of alternative mechanisms that could constitute a competing $[2+2]$ pathway so that we could safely discount these possibilities. These alternatives are discussed below. We have found that all of these mechanisms are disfavored relative to the [4+2] presented above, consistent with our ${ }^{18} \mathrm{O}$-labelled experiments. These computed [2+2] pathways, however, may well be important in Au-catalyzed transformations where 
there is no possibility of a [4+2] pathway occurring. The Au- or Ag-catalyzed formal $[2+2]$ addition of a carbonyl to an alkyne has been proposed to occur in a number of ways. Intra- and intermolecular reactions of aldeyhydes with alkynes catalyzed by $\operatorname{Ag}(\mathrm{I})$ have been proposed by Krische to proceed via an oxetene (or oxete) intermediate. ${ }^{77}$ Yamamoto has instead proposed that an Au-coordinated intermediate is more likely based on the formation of a $\gamma, \delta$-enone byproduct in the carbocyclization of alkynyl ketones. ${ }^{17,27,142}$ DFT calculations suggest that intramolecular reactions of 1,n-enynes catalyzed by $\mathrm{Au}(\mathrm{I})$ proceed via the formation of a cyclopropanyl Au-carbene which then undergoes a 1,2alkyl shift, fragmentation and elimination of Au. ${ }^{174,175}$ We investigated the viability of each of these pathways computationally.

First of all we considered the viability of the [2+2] oxetene mechanism, in which a fourmembered ring is formed from addition of the carbonyl across the alkyne and is followed by an electrocyclic ring opening to give the enone product. The computed structure of the oxetene intermediate and of the ring-opening TS are shown below in Figure 4.
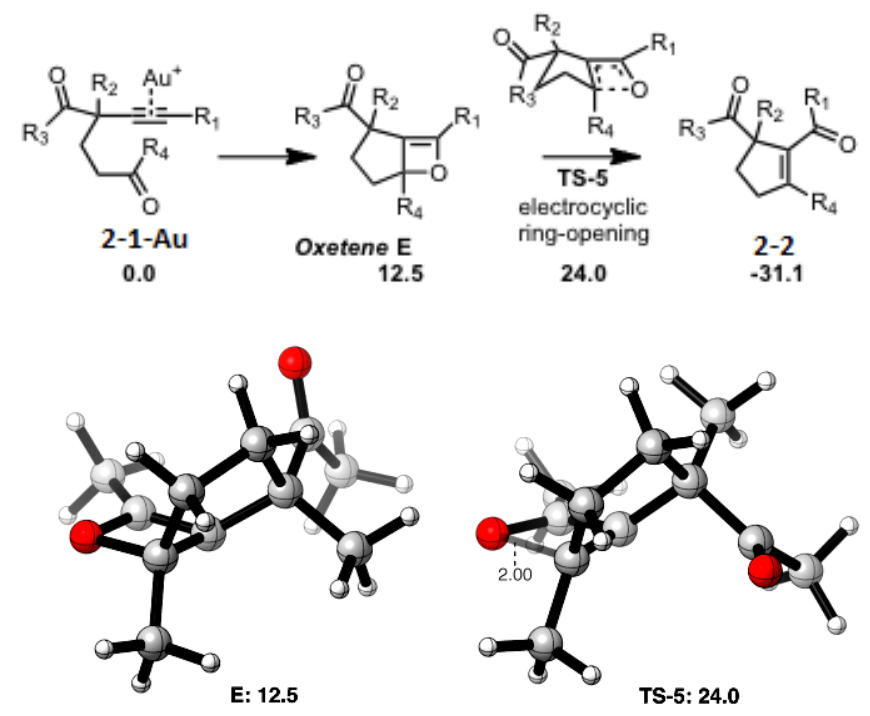

Figure 4: Proposed mechanism and B2PLYP/6-311+G(d,p)/B2PLYP/6-31G(d) computed stationary points for the oxetene pathway. Energies relative to reactant 2-1. Bond breaking distance in $\AA$. 
In accordance with Bredt's rule oxetene $\mathrm{E}$ is highly strained due to the presence of a bridgehead $\mathrm{C}=\mathrm{C}$ bond, lying $12.5 \mathrm{kcal} / \mathrm{mol}$ above the starting material. Therefore any $\mathbf{T S}$ or intermediate lying between $\mathbf{2 - 1}$ and $\mathbf{E}$ is necessarily higher in energy than TS-1 and so disfavored kinetically. Ring opening of $\mathbf{E}$ is possible in the absence of a coordinating metal (e.g. $\mathrm{Au} / \mathrm{Ag}$ ), via the $4 \pi$-electrocyclic TS-5, although this transformation would not be expected to occur readily at room temperature with a relative free energy of 14.0 $\mathrm{kcal} / \mathrm{mol}$. Therefore this pathway is disfavored relative to the [4+2] pathway.

We next considered the possibility of nucleophilic attack of the Au-coordinated alkyne from the carbonyl in a 7-endo-dig fashion, followed by a $4 \pi$ electrocyclic ring closure which leads to the formation of an Au-coordinated bicyclic $\mathbf{G}$, whose ring opening leads to the Au-coordinated enone product (Figure 5). This mechanism represents an Aucatalyzed form of the mechanism shown in Figure 4.
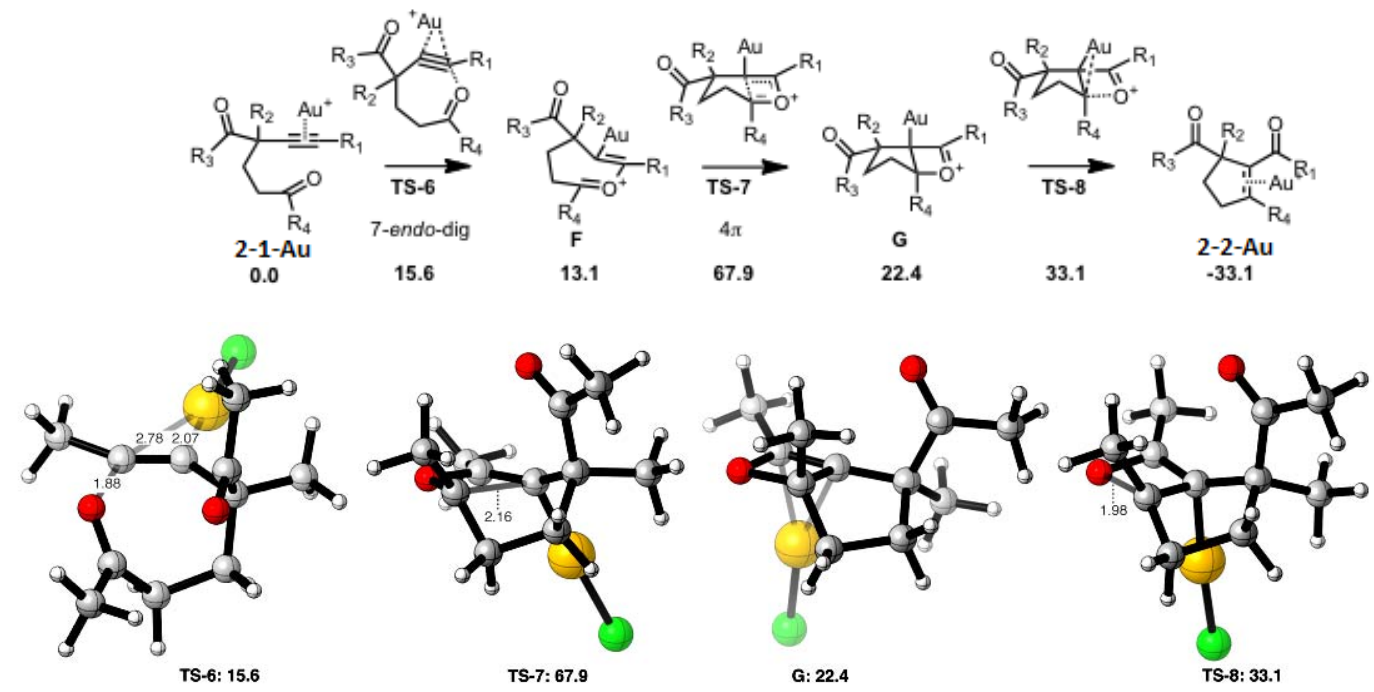

Figure 5: Proposed mechanism and B2PLYP/6-311+G(d,p)/B2PLYP/6-31G(d) computed stationary points for the 7-endo-dig pathway. Energies relative to reactant 2-1. Bond breaking distance in $\AA$.

The initial attack of the Au-coordinated alkyne is disfavored relative to the 5-endo dig attack in the preferred [4+2] mechanism. Furthermore, as a consequence of the anti- 
addition of the carbonyl and Au across the alkyne (syn-addition is impossible in this intramolecular case) and of the conrotatory fashion of the electrocyclic ring closing, the ring junction of $\mathbf{G}$ is necessarily trans-fused. This creates a significant degree of strain, such that ring-closing TS-7 lies $67.9 \mathrm{kcal} / \mathrm{mol}$ above the starting material and the relative energy of $\mathbf{G}$ is $22.4 \mathrm{kcal} / \mathrm{mol}$. The final ring opening step to form the enone product via TS-8 lies uphill at $33.1 \mathrm{kcal} / \mathrm{mol}$. Clearly this mechanism is highly unfavorable.

Based on previous experimental and computational studies of Au-catalyzed rearrangements of enynes, ${ }^{176}$ in which the formation of cyclopropyl Au-carbenes have been invoked, we also considered the possibility of the [2+2] mechanism proceeding via an epoxy Au-carbene as shown in Figure 6. In this mechanism we envisaged that an initial 6-exo cyclization via TS-9 to form $\mathbf{H}$ could then form epoxide $\mathbf{I}$. Fragmentation of this epoxide could then lead to the formal [2+2] addition bicyclic $\mathbf{J}$ that upon ringopening forms the enone product. Compared to the 5-endo cyclization TS-1 the cyclization via 6-exo TS-9 is disfavored, again highlighting the preference for the $[4+2]$ mechanism. We were unable to locate a TS corresponding to the formation of an epoxy Au-carbene at the B2PLYP level of theory; I lies uphill at $23.0 \mathrm{kcal} / \mathrm{mol}$ suggesting that this process would be difficult at room temperature. Rearrangement to $\mathbf{J}$, however, does result in the formation of a much more stable 5,4-bicyclic at $-4.0 \mathrm{kcal} / \mathrm{mol}$ - being cisfused this is much more stable than the corresponding trans-fused bicyclic $\mathbf{G}$ found in Figure 5. The final ring-opening step is relatively facile via TS-11. We have therefore considered three distinct mechanisms which could account for a [2+2] pathway. However, all three are clearly disfavored relative to the computed [4+2] mechanism, in support of our experimental observations. Of the [2+2] pathways considered, none is predicted to 
occur readily at room temperature and a $4 \pi$-electrocylization step (TS-7) can be effectively discounted due to the large energetic barrier.
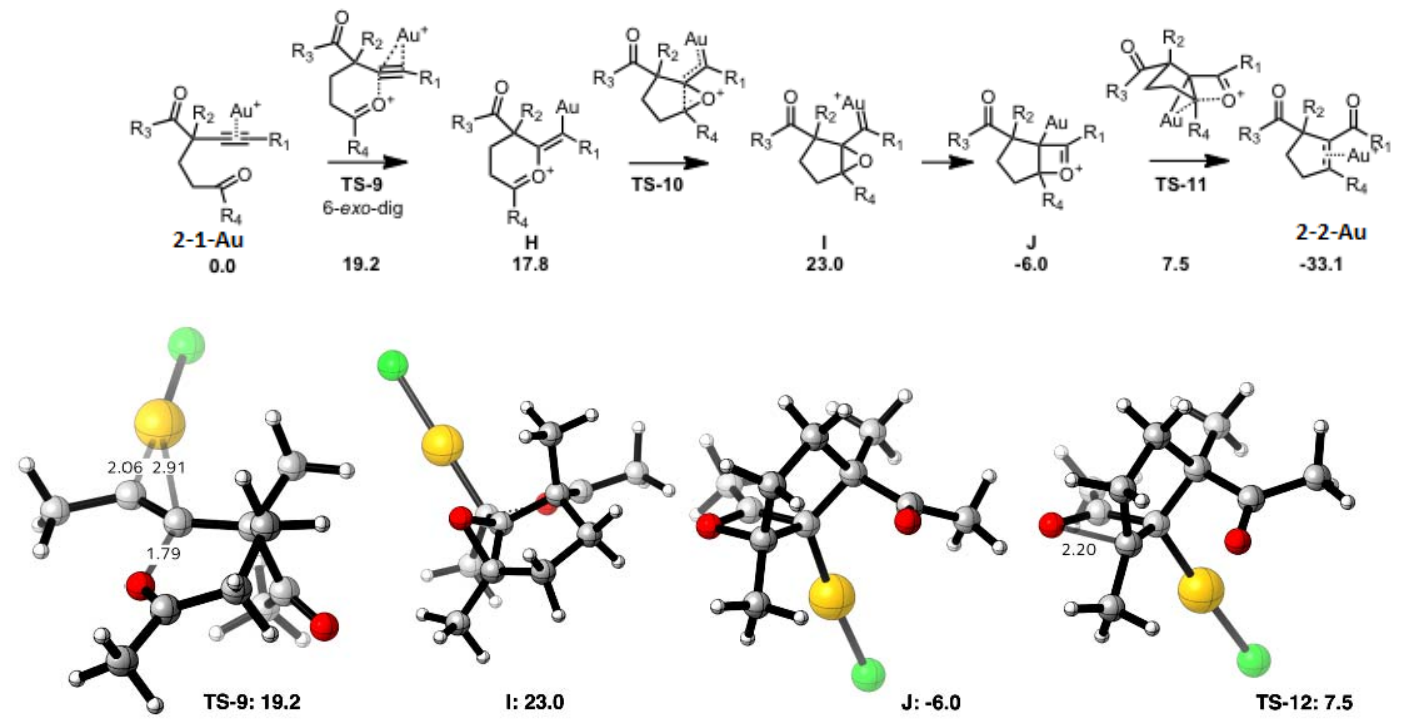

Figure 6: Proposed mechanism and B2PLYP/6-311+G(d,p)/B2PLYP/6-31G(d) computed stationary points for the 6-exo-dig pathway. Energies relative to reactant 1 . Bond breaking distance in $\AA$.

The [4+2] pathway is favored in large part due to the initial selectivity for alkyne addition via a 5-membered TS. To understand why 5-endo-dig attack is so dramatically preferred over 6-exo-dig or 7-endo-dig we computed the activation barrier for some model intraand intermolecular additions of a carbonyl to an Au-coordinated alkyne (Figure 7). Notably, the intermolecular addition of acetone to AuCl-coordinated butyne occurs with an energy barrier of $19.3 \mathrm{kcal} / \mathrm{mol}$, and the barriers of model 6-exo $(20.4 \mathrm{kcal} / \mathrm{mol})$ and 7 endo $(19.9 \mathrm{kcal} / \mathrm{mol})$ intramolecular reactions are very close to this value. In stark contrast the 5-exo mode of attack takes place with a much lower barrier of $9.5 \mathrm{kcal} / \mathrm{mol}$. From this we can conclude that the preference for 5-exo cyclization comes about as TS-1 (Eact $8.8 \mathrm{kcal} / \mathrm{mol}$ ) is remarkably stable rather than any inherent instability of competing pathways proceeding via 6-exo or 7-endo transition states. Acceleration of this ringclosing due to the presence of a quaternary center (Thorpe-Ingold effect) in the five- 
membered ring is only marginal, since replacing this carbon with methylene raises the barrier by just $0.7 \mathrm{kcal} / \mathrm{mol}$. It seems that 5 -exo attack benefits from an almost planar dihedral angle $\left(0.8^{\circ}\right)$ between the alkyne and attacking carbonyl not present in other modes of attack due to the constraints of the 6-membered $\left(18^{\circ}\right)$ or 7 -membered ring $\left(52^{\circ}\right)$, and also in the intermolecular reaction $\left(76^{\circ}\right)$ due to steric demands absent in TS-1. Bond distances and Wiberg bond indices show the 5-exo TS to be earlier, which is a consequence of the increased stability of the cyclized 5-exo product.

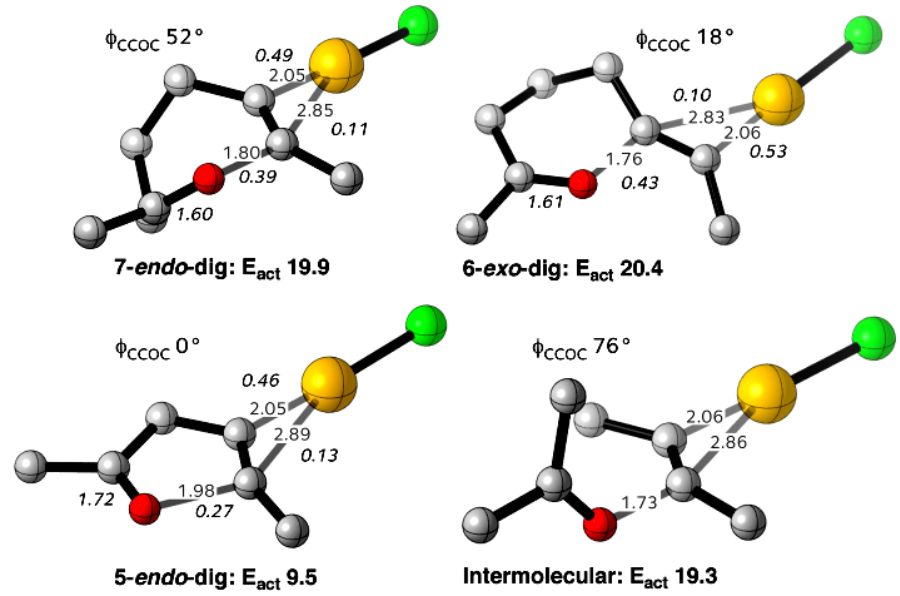

Figure 7: B2PLYP/6-311+G(d,p)/B2PLYP/6-31G(d) (hydrogen-deleted) transition structures for intramolecular and intermolecular cyclization of a ketone onto a Au-coordinated alkyne. Bond distances in $\AA$ with associated Wiberg bond indices italicized. Activation energies relative to Aucoordinated alkyne in $\mathrm{kcal} / \mathrm{mol}$.

\subsection{Summary}

In conclusion, we have developed a synthetic methodology to construct highly substituted cyclopentenyl ketones in very good yield by gold-catalyzed intramolecular alkyne-ketone metathesis of 2-alkynyl-1,5-diketones or 2-alkynyl-5-ketoesters under very mild conditions. The mechanistic investigations of this cycloisomerization using ${ }^{18} \mathrm{O}$ isotopic labeling experiments and quantum chemical calculations revealed that the transformation appears to follow the intramolecular [4+2] cycloaddition of a gold-containing furanium 
intermediate to a carbonyl group. To the best of our knowledge, this is the first example of [4+2] cycloaddition of an oxonium intermediate to a carbonyl group. The work described in this chapter was published in Angew. Chem. Int. Ed. 2010, 49, 9132-9135 and Chem. Eur. J. 2011, 17, 10690-10699.

\subsection{Experimental}

\section{General}

${ }^{1} \mathrm{H}$ and ${ }^{13} \mathrm{C}$ NMR spectra were recorded at 500, 126 (or 400 and 101) $\mathrm{MHz}$ respectively, using $\mathrm{CDCl}_{3}$ as a solvent. The chemical shifts are reported in $\delta(\mathrm{ppm})$ values relative to $\mathrm{CHCl}_{3}\left(\delta 7.26 \mathrm{ppm}\right.$ for ${ }^{1} \mathrm{H}$ NMR and $\delta 77.0 \mathrm{ppm}$ for ${ }^{13} \mathrm{C}$ NMR), multiplicities are indicated by $\mathrm{s}$ (singlet), $\mathrm{d}$ (doublet), $\mathrm{t}$ (triplet), $\mathrm{q}$ (quartet), $\mathrm{p}$ (pentet), h (hextet), $\mathrm{m}$ (multiplet) and br (broad). Coupling constants, $J$, are reported in Hertz. Coupling constants are reported in hertz $(\mathrm{Hz})$. All air and/or moisture sensitive reactions were carried out under argon atmosphere. Solvents (tetrahydrofuran, ether, dichloromethane and DMF) were chemically dried using a commercial solvent purification system. All other reagents and solvents were employed without further purification. The products were purified using a Biotage flash ${ }^{+}$system or Chromatotron apparatus or a regular glass column. TLC was developed on Merck silica gel 60 F254 aluminum sheets. Elemental analysis was performed at Atlantic Microlabs Inc., Norcross, Georgia 30091. High resolution ESI-MS were obtained using a MS-FTICR-MS ${ }^{\mathrm{n}}$ system (LTQ FT, Thermo Electron Corp.) at the CREAM Mass Spectrometry Facility, University of Louisville, Kentucky. 
General procedure for gold-catalyzed oxygen transfer of 2-alkynyl-1,5diketones 2-1 to the corresponding cyclopentenylketones 2-2.

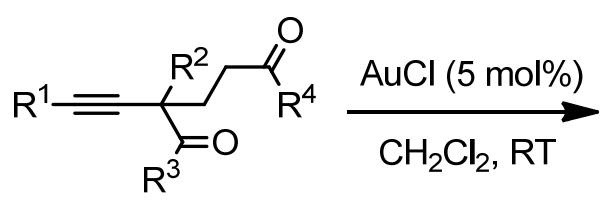

2-1<smiles>[R]C(=O)C1=C([R])CCC1([R])C([R])=O</smiles>

2-2

To a solution of 2-methyl-1-phenyl-2-(phenylethynyl)hexane-1,5-dione 2-1 (0.20 mmol) in dichloromethane $(1.0 \mathrm{~mL})$ was added $\mathrm{AuCl}(0.010 \mathrm{mmol})$. The mixture was stirred for 5 minutes at room temperature. The solvent was removed under reduced pressure and the residue was subjected to a flash column chromatography (eluent: ethyl acetate/n-hexane $=1 / 10$ ) to give product $\mathbf{2 - 2}$ as a colorless oil.

\section{Spectroscopic data of compounds (2-2a to 2-2j)}
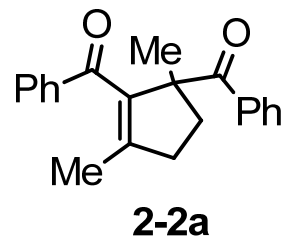

Compound 2-2a: a colorless oil; IR (neat) $\bar{v}$ 2968, 2930, 1673, 1645, 1596, 1446, 1283, $973 \mathrm{~cm}^{-1} ;{ }^{1} \mathrm{H}$ NMR $\left(\mathrm{CDCl}_{3}, 500 \mathrm{MHz}\right) \delta 1.58(3 \mathrm{H}, \mathrm{s}), 1.70(3 \mathrm{H}, \mathrm{s}), 2.14-2.18(1 \mathrm{H}, \mathrm{m})$, 2.48-2.53 (1H, m), 2.56-2.61 (1H, m), 2.76-2.78 (1H, m), 7.39-7.55 (6H, m), 7.48-7.84 $(4 \mathrm{H}, \mathrm{t}, J=7.0 \mathrm{~Hz}) ;{ }^{13} \mathrm{C} \mathrm{NMR}\left(\mathrm{CDCl}_{3}, 126 \mathrm{MHz}\right) \delta 17.2,24.5,36.0,38.5,65.5,128.1$, 128.4, 128.5, 129.3, 131.4, 132.4, 137.3, 139.4, 140.8, 148.7, 196.5, 204.5. Anal. Calcd. For $\mathrm{C}_{21} \mathrm{H}_{20} \mathrm{O}_{2}: \mathrm{C}, 82.86 ; \mathrm{H}, 6.62$. Found: $\mathrm{C}, 82.68 ; \mathrm{H}, 6.76$. 


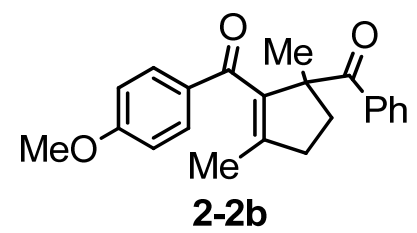

Compound 2-2b: a colorless oil; IR (neat) $\bar{v}$ 2965, 2931, 1722, 1673, 1638, 1598, 1258, $1027 \mathrm{~cm}^{-1} ;{ }^{1} \mathrm{H}$ NMR $\left(\mathrm{CDCl}_{3}, 500 \mathrm{MHz}\right) \delta 1.62(3 \mathrm{H}, \mathrm{s}), 1.66(3 \mathrm{H}, \mathrm{s}), 2.09-2.14(1 \mathrm{H}, \mathrm{m})$, 2.45-2.55 (2H, m), 2.69-2.74 (1H, m), 3.88(3H, s), 6.93-6.95 (2H, d, $J=9.0 \mathrm{~Hz}), 7.36-$ $7.39(2 \mathrm{H}, \mathrm{t}, J=7.5 \mathrm{~Hz}), 7.43-7.46(1 \mathrm{H}, \mathrm{t}, J=7.4 \mathrm{~Hz}), 7.79-7.81(2 \mathrm{H}, \mathrm{d}, J=9.0 \mathrm{~Hz}), 7.84-$ $7.86(2 \mathrm{H}, \mathrm{d}, J=9.0 \mathrm{~Hz}) ;{ }^{13} \mathrm{C} \mathrm{NMR}\left(\mathrm{CDCl}_{3}, 126 \mathrm{MHz}\right) \delta$ 17.1, 22.5, 36.1, 38.2, 55.4, 65.4, 113.6, 128.0, 128.4, 131.2, 131.6, 132.1, 137.6, 140.7, 146.5, 163.2, 195.1, 204.4.

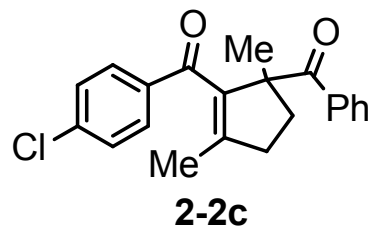

Compound 2-2c: a colorless oil; IR (neat) $\bar{v}$ 2969, 2931, 1671, 1645, 1586, 1446, 1342, 1089, $974 \mathrm{~cm}^{-1} ;{ }^{1} \mathrm{H} \mathrm{NMR}\left(\mathrm{CDCl}_{3}, 500 \mathrm{MHz}\right) \delta 1.58(3 \mathrm{H}, \mathrm{s}), 1.72(3 \mathrm{H}, \mathrm{s}), 2.19-2.22(1 \mathrm{H}$, m), 2.47-2.61 (2H, m), 2.74-2.79 (1H, m), 7.38-7.49 (6H, m), 7.79-7.82 $(4 \mathrm{H}, \mathrm{m}) ;{ }^{13} \mathrm{C}$ NMR $\left(\mathrm{CDCl}_{3}, 126 \mathrm{MHz}\right) \delta 17.1,24.6,36.2,38.3,66.0,128.1,128.5,128.6,130.7,131.7$, 136.8, 137.7, 138.7, 140.3, 148.0, 195.4, 204.3. HRMS (ESI) Calcd. for $\mathrm{C}_{21} \mathrm{H}_{20} \mathrm{ClO}_{2}$ $\left(\mathrm{M}+\mathrm{H}^{+}\right)$requires 339.1146, Found: 339.1152.

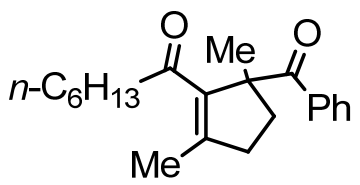

2-2d

Compound 2-2d: a colorless oil; IR (neat) $\bar{v}$ 2955, 2870, 1730, 1677, 1447, 1261, 1176 $973 \mathrm{~cm}^{-1} ;{ }^{1} \mathrm{H} \mathrm{NMR}\left(\mathrm{CDCl}_{3}, 500 \mathrm{MHz}\right) \delta$ 0.82-0.89 (3H, m), 1.09-1.28 (9H, m), 1.41-1.47 
$(2 \mathrm{H}, \mathrm{m}), 1.49(3 \mathrm{H}, \mathrm{s}), 1.85-1.89(1 \mathrm{H}, \mathrm{m}), 2.37-2.50(3 \mathrm{H}, \mathrm{m}), 2.64-2.71(1 \mathrm{H}, \mathrm{m}), 2.81-$ $2.86(1 \mathrm{H}, \mathrm{m}), 7.32-7.35(2 \mathrm{H}, \mathrm{t}, J=7.5 \mathrm{~Hz}), 7.41-7.44(1 \mathrm{H}, \mathrm{t}, J=7.5 \mathrm{~Hz}), 7.78-7.79(2 \mathrm{H}$, d, $J=9.0 \mathrm{~Hz}) ;{ }^{13} \mathrm{C} \mathrm{NMR}\left(\mathrm{CDCl}_{3}, 126 \mathrm{MHz}\right) \delta 13.9,17.4,22.4,23.7,23.8,28.7,31.6$, $35.4,39.5,42.5,62.7,128.3,131.3,132.4,137.4,144.4,150.8,199.7,203.6$.

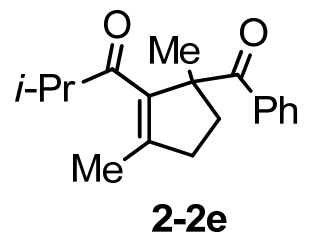

Compound 2-2e: a colorless oil; IR (neat) $\bar{v} 2969,2923,1723,1436,1100,746,693 \mathrm{~cm}^{-}$ ${ }^{1} ;{ }^{1} \mathrm{H}$ NMR $\left(\mathrm{CDCl}_{3}, 500 \mathrm{MHz}\right) \delta$ 0.89-0.91 (3H, d, $\left.J=6.5 \mathrm{~Hz}\right), 0.95-0.97(3 \mathrm{H}, \mathrm{d}, J=6.5$ $\mathrm{Hz}), 1.46(3 \mathrm{H}, \mathrm{s}), 1.82-1.87(1 \mathrm{H}, \mathrm{m}), 2.15(3 \mathrm{H}, \mathrm{s}), 2.36-2.42(1 \mathrm{H}, \mathrm{m}), 2.64-2.69(1 \mathrm{H}, \mathrm{m})$, 2.80-2.86 (1H, m), 2.91-2.96 (1H, m), 7.31-7.34 (2H, t, $J=7.5 \mathrm{~Hz}), 7.39-7.42(1 \mathrm{H}, \mathrm{m})$, 7.76-7.77 (2H, d, $J=7.5 \mathrm{~Hz}) ;{ }^{13} \mathrm{C} \mathrm{NMR}\left(\mathrm{CDCl}_{3}, 126 \mathrm{MHz}\right) \delta 17.2,18.3,18.6,23.8,35.2$, $38.2,39.7,62.8,127.9,128.3,131.1,137.5,143.5,150.6,203.5,203.7$. HRMS (ESI) Calcd. for $\mathrm{C}_{18} \mathrm{H}_{23} \mathrm{O}_{2}\left(\mathrm{M}+\mathrm{H}^{+}\right)$requires 271.1693, Found: 271.1696.

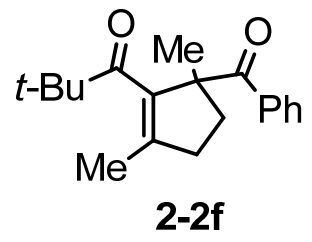

Compound 2-2f: a colorless oil; IR (neat) $\bar{v}$ 2971, 2933, 1716, 1673, 1447, 1263, 975, $712 \mathrm{~cm}^{-1} ;{ }^{1} \mathrm{H} \mathrm{NMR}\left(\mathrm{CDCl}_{3}, 500 \mathrm{MHz}\right) \delta 1.26(9 \mathrm{H}, \mathrm{s}), 1.51(3 \mathrm{H}, \mathrm{s}), 1.84(3 \mathrm{H}, \mathrm{s}), 1.96-$ $2.01(1 \mathrm{H}, \mathrm{m}), 2.28-2.34(2 \mathrm{H}, \mathrm{m}), 2.52-2.56(1 \mathrm{H}, \mathrm{m}), 7.34-7.37(2 \mathrm{H}, \mathrm{t}, J=7.5 \mathrm{~Hz}), 7.42-$ $7.45(1 \mathrm{H}, \mathrm{t}, J=7.5 \mathrm{~Hz}), 7.71-7.73(2 \mathrm{H}, \mathrm{d}, J=7.5 \mathrm{~Hz}) ;{ }^{13} \mathrm{C} \mathrm{NMR}\left(\mathrm{CDCl}_{3}, 126 \mathrm{MHz}\right) \delta$ 17.4, 24.6, 27.6, 36.1, 37.7, 43.9, 66.7, 127.9, 128.3, 131.1, 137.9, 139.5, 141.4, 204.7, 215.2. HRMS (ESI) Calcd. for $\mathrm{C}_{19} \mathrm{H}_{25} \mathrm{O}_{2}\left(\mathrm{M}+\mathrm{H}^{+}\right)$requires 285.1849, Found: 285.1850 . 


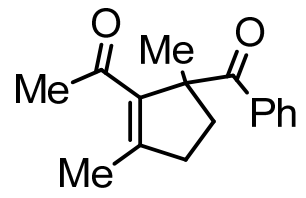

\section{$2-2 g$}

Compound 2-2g: a white solid; IR (neat) $\bar{v}$ 2970, 2931, 1674, 1652, 1430, 1262, 972, $710 \mathrm{~cm}^{-1} ;{ }^{1} \mathrm{H} \mathrm{NMR}\left(\mathrm{CDCl}_{3}, 400 \mathrm{MHz}\right) \delta 1.46(3 \mathrm{H}, \mathrm{s}), 1.82-1.88(1 \mathrm{H}, \mathrm{m}), 2.14(3 \mathrm{H}, \mathrm{s})$, $2.19(3 \mathrm{H}, \mathrm{s}), 2.33-2.41(1 \mathrm{H}, \mathrm{m}), 2.62-2.69(1 \mathrm{H}, \mathrm{m}), 2.78-2.87(1 \mathrm{H}, \mathrm{m}), 7.29-7.33(2 \mathrm{H}, \mathrm{t}, J$ $=7.5 \mathrm{~Hz}), 7.38-7.42(1 \mathrm{H}, \mathrm{t}, J=7.2 \mathrm{~Hz}), 7.75-7.77(2 \mathrm{H}, \mathrm{d}, J=7.6 \mathrm{~Hz}) ;{ }^{13} \mathrm{C} \mathrm{NMR}\left(\mathrm{CDCl}_{3}\right.$, $101 \mathrm{MHz}) \delta 17.4,23.7,30.7,35.4,39.5,62.6,128.0,128.3,131.4,137.3,144.5,152.4$, 196.8, 203.6. Anal. Calcd. For $\mathrm{C}_{21} \mathrm{H}_{20} \mathrm{O}_{2}$ : C, 79.31; H, 7.49. Found: C, 79.43; H, 7.64.

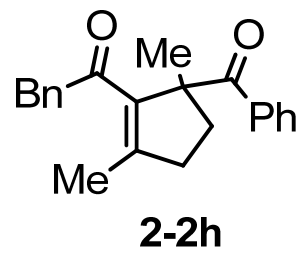

Compound 2-2h: a colorless oil; IR (neat) $\bar{v}$ 2952, 2868, 1676, 1652, 1446, 1263, 973, $707 \mathrm{~cm}^{-1} ;{ }^{1} \mathrm{H}$ NMR $\left(\mathrm{CDCl}_{3}, 500 \mathrm{MHz}\right) \delta 1.53(3 \mathrm{H}, \mathrm{s}), 1.91-1.95(1 \mathrm{H}, \mathrm{m}), 2.19(3 \mathrm{H}, \mathrm{s})$, 2.44-2.48 (1H, m), 2.67-2.73 (1H, m), 2.82-2.90 (1H, m), $3.86(2 \mathrm{H}, \mathrm{s}), 6.95-6.96(2 \mathrm{H}, \mathrm{d}$, $J=7.0 \mathrm{~Hz}), 7.10-7.27(3 \mathrm{H}, \mathrm{m}), 7.35-7.38(2 \mathrm{H}, \mathrm{t}, J=7.5 \mathrm{~Hz}), 7.45-7.48(1 \mathrm{H}, \mathrm{t}, J=7.5$ $\mathrm{Hz}), 7.79-7.81(2 \mathrm{H}, \mathrm{d}, J=8.0 \mathrm{~Hz}) ;{ }^{13} \mathrm{C} \mathrm{NMR}\left(\mathrm{CDCl}_{3}, 126 \mathrm{MHz}\right) \delta 15.5,21.9,33.6,37.5$, $46.7,61.1,124.6,126.0,126.3,126.4,127.4,129.3,132.2,135.4,142.0,149.6,195.0$, 201.6. HRMS (ESI) Calcd. for $\mathrm{C}_{22} \mathrm{H}_{23} \mathrm{O}_{2}\left(\mathrm{M}+\mathrm{H}^{+}\right)$requires 319.1693, Found: 319.1693 .

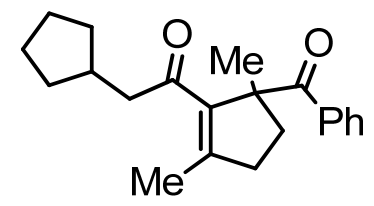

2-2i 
Compound 2-2i: a colorless oil; IR (neat) $\bar{v}$ 2951, 2868, 1676, 1652, 1446, 1263, 973, $707 \mathrm{~cm}^{-1} ;{ }^{1} \mathrm{H}$ NMR $\left(\mathrm{CDCl}_{3}, 500 \mathrm{MHz}\right) \delta$ 0.81-0.94 (2H, m), 1.42-1.51 (7H, m), 1.58-1.65 $(2 \mathrm{H}, \mathrm{m}), 1.81-1.86(1 \mathrm{H}, \mathrm{m}), 2.09-2.15(4 \mathrm{H}, \mathrm{m}), 2.36-2.42(1 \mathrm{H}, \mathrm{m}), 2.51-2.52(2 \mathrm{H}, \mathrm{d}, J=$ $7.0 \mathrm{~Hz}), 2.64-2.70(1 \mathrm{H}, \mathrm{m}), 2.80-2.86(1 \mathrm{H}, \mathrm{m}), 7.31-7.33(2 \mathrm{H}, \mathrm{dt}, J=7.0,1.0 \mathrm{~Hz}), 7.38-$ $7.42(1 \mathrm{H}, \mathrm{dd}, J=7.0,1.0 \mathrm{~Hz}), 7.77-7.78(2 \mathrm{H}, \mathrm{d}, J=8.5 \mathrm{~Hz}),{ }^{13} \mathrm{C} \mathrm{NMR}\left(\mathrm{CDCl}_{3}, 126 \mathrm{MHz}\right)$ $\delta 13.5,19.7,20.8,28.2,28.4,31.3,35.6,44.7,58.6,123.9,124.3,127.2,133.4,140.6$, 146.8, 195.1, 199.4. HRMS (ESI) Calcd. for $\mathrm{C}_{21} \mathrm{H}_{27} \mathrm{O}_{2}\left(\mathrm{M}+\mathrm{H}^{+}\right)$requires 311.2006, Found: 311.2008 .

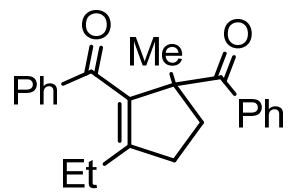

2-2j

Compound 2-2j: a colorless oil; IR (neat) $\bar{v}$ 2970, 2935, 1673, 1644, 1596, 1447, 1283, 1176, 976, $695 \mathrm{~cm}^{-1} ;{ }^{1} \mathrm{H} \mathrm{NMR}\left(\mathrm{CDCl}_{3}, 400 \mathrm{MHz}\right) \delta 0.87-0.91(3 \mathrm{H}, \mathrm{t}, J=7.6 \mathrm{~Hz}), 1.65$ $(3 \mathrm{H}, \mathrm{s}), 1.91-1.97(2 \mathrm{H}, \mathrm{m}), 2.09-2.16(1 \mathrm{H}, \mathrm{m}), 2.41-2.48(1 \mathrm{H}, \mathrm{m}), 2.53-2.61(1 \mathrm{H}, \mathrm{m})$, 2.68-2.75( 1H, m), 7.34-7.44 (5H, m), 7.50-7.54 (1H, m), 7.78-7.84 (4H, m); ${ }^{13} \mathrm{C}$ NMR $\left(\mathrm{CDCl}_{3}, 101 \mathrm{MHz}\right) \delta 12.0,23.9,24.4,34.8,35.8,65.5,128.0,128.3,128.4,129.1,131.5$, 132.4, 137.5, 139.5, 140.0, 152.9, 196.7, 204.4. HRMS (ESI) Calcd. for $\mathrm{C}_{22} \mathrm{H}_{22} \mathrm{O}_{2} \mathrm{Na}$ $\left(\mathrm{M}+\mathrm{Na}^{+}\right)$requires 341.1512, Found: 341.1512.

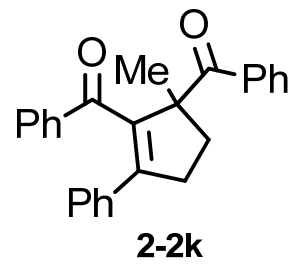

Compound 2-2k: ${ }^{1} \mathrm{H}$ NMR $\left(\mathrm{CDCl}_{3}, 400 \mathrm{MHz}\right) \delta 7.90(\mathrm{~d}, J=7.2 \mathrm{~Hz}, 2 \mathrm{H}), 7.65(\mathrm{~d}, J=7.6$ Hz, 2H), 7.44 (t, $J=7.2 \mathrm{~Hz}, 1 \mathrm{H}), 7.37$ (t, $J=7.6 \mathrm{~Hz}, 2 \mathrm{H}), 7.24$ (t, $J=7.6 \mathrm{~Hz}, 1 \mathrm{H}), 7.05-$ 
$7.13(\mathrm{~m}, 7 \mathrm{H}), 3.22-3.29(\mathrm{~m}, 1 \mathrm{H}), 3.00-3.09(\mathrm{~m}, 1 \mathrm{H}), 2.64-2.72(\mathrm{~m}, 1 \mathrm{H}), 2.22-2.28(\mathrm{~m}$, 1H), $1.73(\mathrm{~s}, 3 \mathrm{H}) .{ }^{13} \mathrm{C} \mathrm{NMR}\left(\mathrm{CDCl}_{3}, 100 \mathrm{MHz}\right) \delta 203.3,196.5,148.6,141.2,137.4$, $137.3,135.6,132.1,131.5,129.5,128.6,128.4,128.3,128.1,127.9,127.7,66.2,36.4$, 35.8, 24.3. HRMS m/z (ESI) calcd for $\mathrm{C}_{26} \mathrm{H}_{23} \mathrm{O}_{2}\left(\mathrm{M}+\mathrm{H}^{+}\right)$367.1693, found 367.1687; IR (neat) $\bar{v} 1674,1637,1447,1277,1251,968,725,693 \mathrm{~cm}^{-1}$.

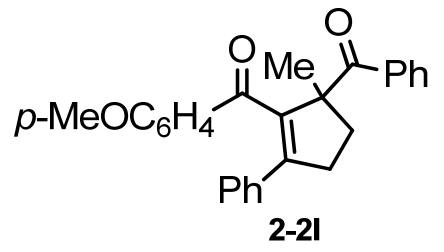

Compound 2-2I: ${ }^{1} \mathrm{H}$ NMR $\left(\mathrm{CDCl}_{3}, 400 \mathrm{MHz}\right) \delta 7.90(\mathrm{~d}, J=8.0 \mathrm{~Hz}, 2 \mathrm{H}), 7.67(\mathrm{~d}, J=8.0$ Hz, 2H), 7.42 (t, $J=6.8 \mathrm{~Hz}, 1 \mathrm{H}), 7.35$ (t, $J=6.8 \mathrm{~Hz}, 2 \mathrm{H}), 7.15$ (brs, 2H), 7.09 (brs, 3H), $6.62(\mathrm{~d}, J=8.4 \mathrm{~Hz}, 2 \mathrm{H}), 3.70(\mathrm{~s}, 3 \mathrm{H}), 3.02-3.29(\mathrm{~m}, 1 \mathrm{H}), 2.95-3.01(\mathrm{~m}, 1 \mathrm{H}), 2.61-2.69$ (m, 1H), 2.17-2.23 (m, 1H), $1.66(\mathrm{~s}, 3 \mathrm{H}) .{ }^{13} \mathrm{C} \mathrm{NMR}\left(\mathrm{CDCl}_{3}, 100 \mathrm{MHz}\right) \delta$ 203.4, 195.1, $162.9,146.7,141.1,137.7,135.7,131.8,131.3,130.1,128.6,128.3,128.2,128.1,113.1$, 65.9, 55.2, 36.1, 35.7, 24.2. HRMS m/z (ESI) calcd for $\mathrm{C}_{27} \mathrm{H}_{25} \mathrm{O}_{3}\left(\mathrm{M}+\mathrm{H}^{+}\right)$397.1798, found 397.1790, IR (neat) $\bar{v}$ 1675, 1637, 1596, 1508, 1334, 1255, 1162, 763, $698 \mathrm{~cm}^{-1}$.

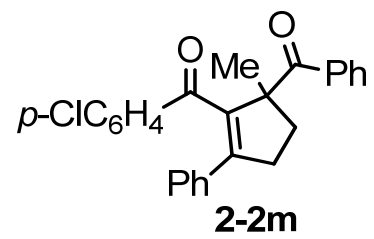

Compound 2-2m: ${ }^{1} \mathrm{H}$ NMR $\left(\mathrm{CDCl}_{3}, 400 \mathrm{MHz}\right) \delta 7.88(\mathrm{~d}, J=8.0 \mathrm{~Hz}, 2 \mathrm{H}), 7.60(\mathrm{~d}, J=8.0$ $\mathrm{Hz}, 2 \mathrm{H}), 7.46(\mathrm{t}, J=7.2 \mathrm{~Hz}, 1 \mathrm{H}), 7.38(\mathrm{t}, J=7.6 \mathrm{~Hz}, 2 \mathrm{H}), 7.10$ (brs, $7 \mathrm{H}), 3.17-3.25(\mathrm{~m}$, 1H), 3.04-3.12 (m, 1H), 2.64-2.72 (m, 1H), 2.26-2.72 (m, 1H), 1.77 (s, 3H). ${ }^{13} \mathrm{C}$ NMR $\left(\mathrm{CDCl}_{3}, 100 \mathrm{MHz}\right) \delta 203.4,195.4,148.5,140.8,138.3,136.8,135.8,135.4,131.7,130.8$ 128.7, 128.6, 128.3, 128.2, 128.1, 128.0, 66.8, 36.3, 36.0, 24.6. HRMS m/z (ESI) calcd 
for $\mathrm{C}_{26} \mathrm{H}_{22} \mathrm{O}_{2} \mathrm{Cl}\left(\mathrm{M}+\mathrm{H}^{+}\right)$401.1303, found 401.1297. IR (neat) $\bar{v} 1674,1637,1576,1275$, $1251,1089,968,747,697 \mathrm{~cm}^{-1}$.

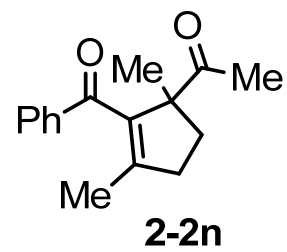

Compound 2-2n: ${ }^{1} \mathrm{H}$ NMR (CDCl $\left.3,400 \mathrm{MHz}\right) \delta 7.75(\mathrm{~d}, J=7.6 \mathrm{~Hz}, 2 \mathrm{H}), 7.52(\mathrm{t}, J=7.2$ Hz, 1H), 7.42 (t, $J=7.6 \mathrm{~Hz}, 2 \mathrm{H}), 2.61-2.66(\mathrm{~m}, 2 \mathrm{H}), 2.09-2.17(\mathrm{~m}, 4 \mathrm{H}), 1.78-1.83$ (m, 1H), $1.56(\mathrm{~s}, 3 \mathrm{H}), 1.38(\mathrm{~s}, 3 \mathrm{H}) .{ }^{13} \mathrm{C} \mathrm{NMR}\left(\mathrm{CDCl}_{3}, 100 \mathrm{MHz}\right) \delta 210.7,196.1,150.1,140.4$, 139.2, 132.6, 129.0, 128.5, 64.8, 38.7, 34.9, 25.7, 22.0, 17.3. HRMS m/z (ESI) calcd for $\mathrm{C}_{16} \mathrm{H}_{19} \mathrm{O}_{2}\left(\mathrm{M}+\mathrm{H}^{+}\right)$243.1380, found 243.1381. IR (neat) $\bar{v}$ 1706, 1640, 1447, 1346, 1281, $739,696 \mathrm{~cm}^{-1}$.

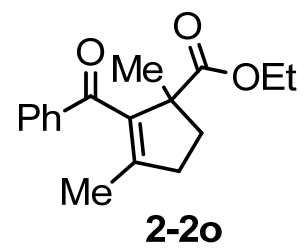

Compound 2-2o: A colorless oil; ${ }^{1} \mathrm{H}$ NMR $\left(\mathrm{CDCl}_{3}, 400 \mathrm{MHz}\right) \delta 1.02(3 \mathrm{H}, \mathrm{t}, J=7.2 \mathrm{~Hz})$, $1.46(6 \mathrm{H}, \mathrm{s}), 1.81(1 \mathrm{H}, \mathrm{m}), 2.27(1 \mathrm{H}, \mathrm{m}), 2.51(2 \mathrm{H}, \mathrm{t}, J=7.2 \mathrm{~Hz}), 3.98(2 \mathrm{H}, \mathrm{q}, J=7.2$ $\mathrm{Hz}), 7.36(2 \mathrm{H}, \mathrm{t}, J=7.6 \mathrm{~Hz}), 7.46(1 \mathrm{H}, \mathrm{t}, J=6.8 \mathrm{~Hz}), 7.71(2 \mathrm{H}, \mathrm{d}, J=7.6 \mathrm{~Hz}) ;{ }^{13} \mathrm{C}$ NMR $\left(\mathrm{CDCl}_{3}, 126 \mathrm{MHz}\right)$ 13.9, 17.1, 22.7, 36.5, 37.8, 58.7, 60.6, 128.3, 129.1, 132.4, 139.2, 139.4, 149.1, 176.2, 196.5. HRMS (ESI) Calcd. for $\mathrm{C}_{17} \mathrm{H}_{20} \mathrm{NaO}_{3}\left(\mathrm{M}+\mathrm{H}^{+}\right)$requires 295.1305, Found: 295.6667.

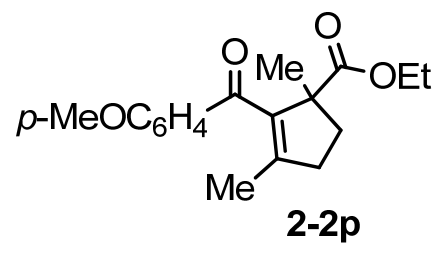


Compound 2-2p: A colorless oil; ${ }^{1} \mathrm{H}$ NMR $\left(\mathrm{CDCl}_{3}, 400 \mathrm{MHz}\right) \delta 1.00(3 \mathrm{H}, \mathrm{t}, J=7.0 \mathrm{~Hz})$, $1.44(3 \mathrm{H}, \mathrm{s}), 1.50(3 \mathrm{H}, \mathrm{s}), 1.79(1 \mathrm{H}, \mathrm{m}), 2.27(1 \mathrm{H}, \mathrm{m}), 2.48(2 \mathrm{H}, \mathrm{m}), 3.79(3 \mathrm{H}, \mathrm{s}), 3.96$ $(2 \mathrm{H}, \mathrm{q}, J=7.2 \mathrm{~Hz}), 6.84(2 \mathrm{H}, \mathrm{d}, J=8.8 \mathrm{~Hz}), 7.73(2 \mathrm{H}, \mathrm{d}, J=8.8 \mathrm{~Hz}) ;{ }^{13} \mathrm{C} \mathrm{NMR}\left(\mathrm{CDCl}_{3}\right.$, $100 \mathrm{MHz}) 13.9,16.9,22.9,36.5,37.6,55.4,58.9,60.6,113.6,131.6,132.0,139.0,147.2$, 163.2, 176.3, 195.3. HRMS (ESI) Calcd. for $\mathrm{C}_{18} \mathrm{H}_{22} \mathrm{NaO}_{4}\left(\mathrm{M}+\mathrm{H}^{+}\right)$requires 325.1410, Found: 325.5833 .

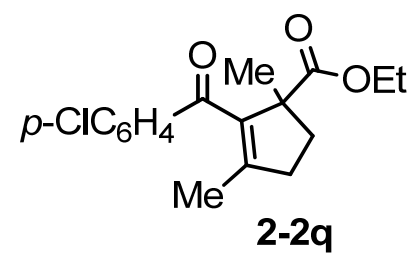

Compound 2-2q: A colorless oil; ${ }^{1} \mathrm{H}$ NMR $\left(\mathrm{CDCl}_{3}, 400 \mathrm{MHz}\right) \delta 1.02(3 \mathrm{H}, \mathrm{m}), 1.46(6 \mathrm{H}$, m), $1.81(1 \mathrm{H}, \mathrm{m}), 2.24(1 \mathrm{H}, \mathrm{m}), 2.49(2 \mathrm{H}, \mathrm{t}, J=6.8 \mathrm{~Hz}), 3.97(2 \mathrm{H}, \mathrm{m}), 7.33(2 \mathrm{H}, \mathrm{m}), 7.68$ $(2 \mathrm{H}, \mathrm{m}) ;{ }^{13} \mathrm{C} \mathrm{NMR}\left(\mathrm{CDCl}_{3}, 100 \mathrm{MHz}\right)$ 14.0, 17.1, 22.7, 36.5, 37.8, 58.9, 60.7, 128.7, 130.5, 137.7, 138.8, 138.9, 149.3, 176.1, 195.2. HRMS (ESI) Calcd. for $\mathrm{C}_{17} \mathrm{H}_{19} \mathrm{ClO}_{3}$ $\left(\mathrm{M}+\mathrm{H}^{+}\right)$requires 329.0915, Found: 329.7500.

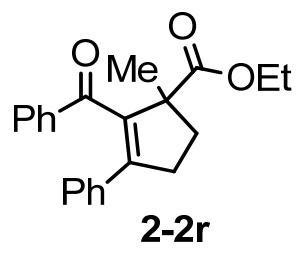

Compound 2-2r: ${ }^{1} \mathrm{H}$ NMR $\left(\mathrm{CDCl}_{3}, 400 \mathrm{MHz}\right) \delta 7.65(\mathrm{~d}, J=8.0 \mathrm{~Hz}, 2 \mathrm{H}), 7.26(\mathrm{t}, J=7.6$ $\mathrm{Hz}, 1 \mathrm{H}), 7.09-7.13(\mathrm{~m}, 4 \mathrm{H}), 7.03-7.06(\mathrm{~m}, 3 \mathrm{H}), 3.93-4.05(\mathrm{~m}, 2 \mathrm{H}), 3.10-3.18(\mathrm{~m}, 1 \mathrm{H})$, 2.92-2.98 (m, 1H), 2.54-2.59 (m, 1H), 2.00-2.06 (m, 1H), 1.62 (s, 3H), 1.00 (t, $J=7.2 \mathrm{~Hz}$, 3H). ${ }^{13} \mathrm{C} \mathrm{NMR}\left(\mathrm{CDCl}_{3}, 100 \mathrm{MHz}\right) \delta 197.0,175.7,148.7,139.4,137.3,135.6,132.3$, $129.4,128.2,128.2,127.9,127.8,60.8,59.9,36.4,35.7,23.1,13.8$. HRMS $m / z$ (ESI) 
calcd for $\mathrm{C}_{22} \mathrm{H}_{23} \mathrm{O}_{3}\left(\mathrm{M}+\mathrm{H}^{+}\right)$335.1642, found 335.1637. IR (neat) $\bar{v}$ 1731, 1646, 1447 , $1279,1254,1173,1093,696 \mathrm{~cm}^{-1}$.

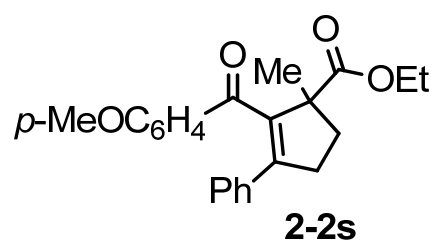

Compound 2-2s: ${ }^{1} \mathrm{H}$ NMR $\left(\mathrm{CDCl}_{3}, 400 \mathrm{MHz}\right) \delta 7.67(\mathrm{~d}, J=8.8 \mathrm{~Hz}, 2 \mathrm{H}), 7.14$ (brs, $\left.2 \mathrm{H}\right)$, 7.07 (brs, 3H), 6.64 (d, J = 8.4 Hz, 2H), 3.91-4.05 (m, 2H), 3.72 (s, 3H), 3.10-3.18 (m, $1 \mathrm{H}), 2.87-2.94(\mathrm{~m}, 1 \mathrm{H}), 2.54-2.61(\mathrm{~m}, 1 \mathrm{H}), 1.97-2.04(\mathrm{~m}, 1 \mathrm{H}), 1.58(\mathrm{~s}, 3 \mathrm{H}), 1.00(\mathrm{t}, J=$ $7.2 \mathrm{~Hz}, 3 \mathrm{H}) .{ }^{13} \mathrm{C} \mathrm{NMR}\left(\mathrm{CDCl}_{3}, 100 \mathrm{MHz}\right) \delta 195.7,175.7,163.0,146.8,139.2,135.7$, $131.8,130.3,128.2,128.1,127.9,113.1,60.8,60.0,55.3,36.3,35.4,23.2,13.8$. HRMS $m / z$ (ESI) calcd for $\mathrm{C}_{23} \mathrm{H}_{25} \mathrm{O}_{4}\left(\mathrm{M}+\mathrm{H}^{+}\right) 365.1747$, found 365.1741. IR (neat) $\bar{v} 1729,1637$, $1597,1507,1256,1162,1093,1027,760,697 \mathrm{~cm}^{-1}$.

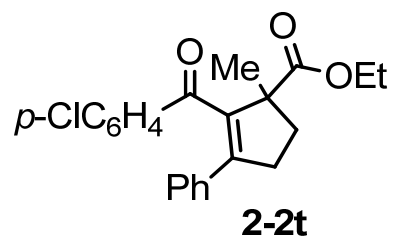

Compound 2-2t: ${ }^{1} \mathrm{H}$ NMR $\left(\mathrm{CDCl}_{3}, 400 \mathrm{MHz}\right) \delta 7.59$ (d, J = 7.6 Hz, 2H), 7.08 (brs, 7H), 3.95-4.07 (m, 2H), 3.10-3.17 (m, 1H), 2.88-3.10 (m, 1H), 2.51-2.58 (m, 1H), 2.00-2.06 (m, 1H), $1.62(\mathrm{~s}, 3 \mathrm{H}), 1.02(\mathrm{t}, J=6.8 \mathrm{~Hz}, 3 \mathrm{H}) .{ }^{13} \mathrm{C} \mathrm{NMR}\left(\mathrm{CDCl}_{3}, 125 \mathrm{MHz}\right) \delta$ 195.7, $175.6,149.2,139.1,138.5,135.8,135.4,130.8,128.6,128.2,128.1,128.1,60.8,60.1$, 36.5, 35.7, 23.2, 13.9. HRMS m/z (ESI) calcd for $\mathrm{C}_{22} \mathrm{H}_{22} \mathrm{O}_{3} \mathrm{Cl}\left(\mathrm{M}+\mathrm{H}^{+}\right) 369.1252$, found 369.1248. IR (neat) v 1732, 1646, 1278, 1253, 1166, 1091, 860, 755, $697 \mathrm{~cm}^{-1}$. 


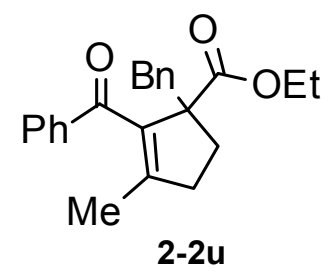

Compound 2-2u: ${ }^{1} \mathrm{H}$ NMR $\left(\mathrm{CDCl}_{3}, 500 \mathrm{MHz}\right) \delta 7.85(\mathrm{~d}, J=7.5 \mathrm{~Hz}, 2 \mathrm{H}), 7.55(\mathrm{t}, J=8.0$ Hz, 1H), 7.45 (t, $J=8.0 \mathrm{~Hz}, 2 \mathrm{H}), 7.25$ (brs, 2H), 7.20 (brs, 3H), 4.00-4.08 (m, 2H), 3.64 (d, $J=13.0 \mathrm{~Hz}, 1 \mathrm{H}), 3.24(\mathrm{~d}, J=13.0 \mathrm{~Hz}, 1 \mathrm{H}), 2.18-2.27(\mathrm{~m}, 1 \mathrm{H}), 2.11-2.16(\mathrm{~m}, 2 \mathrm{H})$, 1.36-1.40 (m, 4H), $1.04(\mathrm{t}, J=7.0 \mathrm{~Hz}, 3 \mathrm{H}) .{ }^{13} \mathrm{C} \mathrm{NMR}\left(\mathrm{CDCl}_{3}, 125 \mathrm{MHz}\right) \delta$ 197.3, 175.7, $151.5,139.3,137.8,135.7,132.5,130.6,129.3,128.3,127.6,126.2,64.2,60.8,41.5,37.7$, 32.6, 16.8, 13.8. HRMS m/z (ESI) calcd for $\mathrm{C}_{23} \mathrm{H}_{25} \mathrm{O}_{3}\left(\mathrm{M}+\mathrm{H}^{+}\right) 349.1798$, found 349.1799. IR (neat) $\bar{v} 1728,1643,1447,1280,1238,1172,1074,703 \mathrm{~cm}^{-1}$.

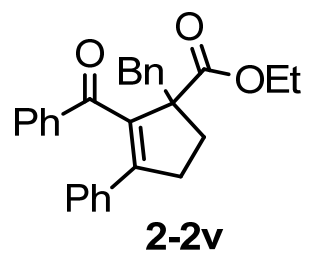

Compound 2-2v: ${ }^{1} \mathrm{H}$ NMR $\left(\mathrm{CDCl}_{3}, 400 \mathrm{MHz}\right) \delta 7.68(\mathrm{~d}, J=7.2 \mathrm{~Hz}, 2 \mathrm{H}), 7.38(\mathrm{~d}, J=6.8$ Hz, 2H), 7.28 (t, $J=7.6 \mathrm{~Hz}, 1 \mathrm{H}), 7.13-7.25$ (m, 6H), 7.01 (brs, 4H), 3.88-4.03 (m, 2H), $3.70(\mathrm{~d}, J=13.6 \mathrm{~Hz}, 1 \mathrm{H}), 3.33(\mathrm{~d}, J=13.6 \mathrm{~Hz}, 1 \mathrm{H}), 2.75-2.83(\mathrm{~m}, 1 \mathrm{H}), 2.46-2.54(\mathrm{~m}$, 1H), 2.27-2.32 (m, $1 \mathrm{H}), 1.77-1.86(\mathrm{~m}, 1 \mathrm{H}), 0.94(\mathrm{t}, J=6.8 \mathrm{~Hz}, 3 \mathrm{H}) .{ }^{13} \mathrm{C} \mathrm{NMR}\left(\mathrm{CDCl}_{3}\right.$, $100 \mathrm{MHz}) \delta$ 197.8, 175.1, 151.1, 137.5, 136.2, 135.6, 132.2, 130.6, 129.5, 128.2, 128.0, $127.8,127.8,127.7,126.5,65.3,61.0,42.3,35.7,32.7,13.7$. HRMS m/z (ESI) calcd for $\mathrm{C}_{28} \mathrm{H}_{27} \mathrm{O} 3\left(\mathrm{M}+\mathrm{H}^{+}\right) 411.1955$, found 411.1949. IR (neat) $\bar{v} 1727,1642,1447,1278,1247$, $1175,697 \mathrm{~cm}^{-1}$. 
${ }^{1} \mathrm{H},{ }^{13} \mathrm{C},{ }^{19} \mathrm{~F}$ Spectra of compound 2-2a and 2-20.

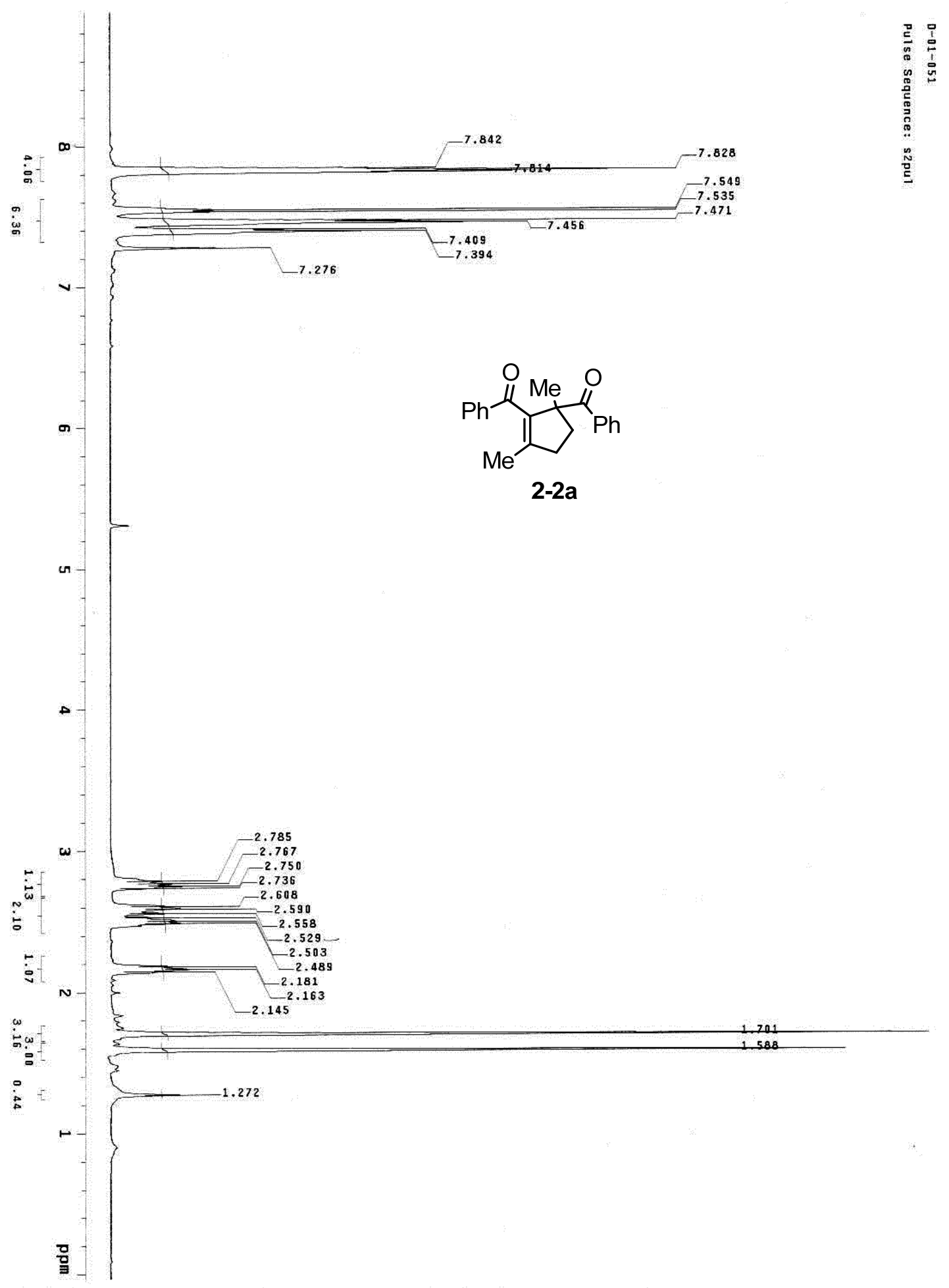




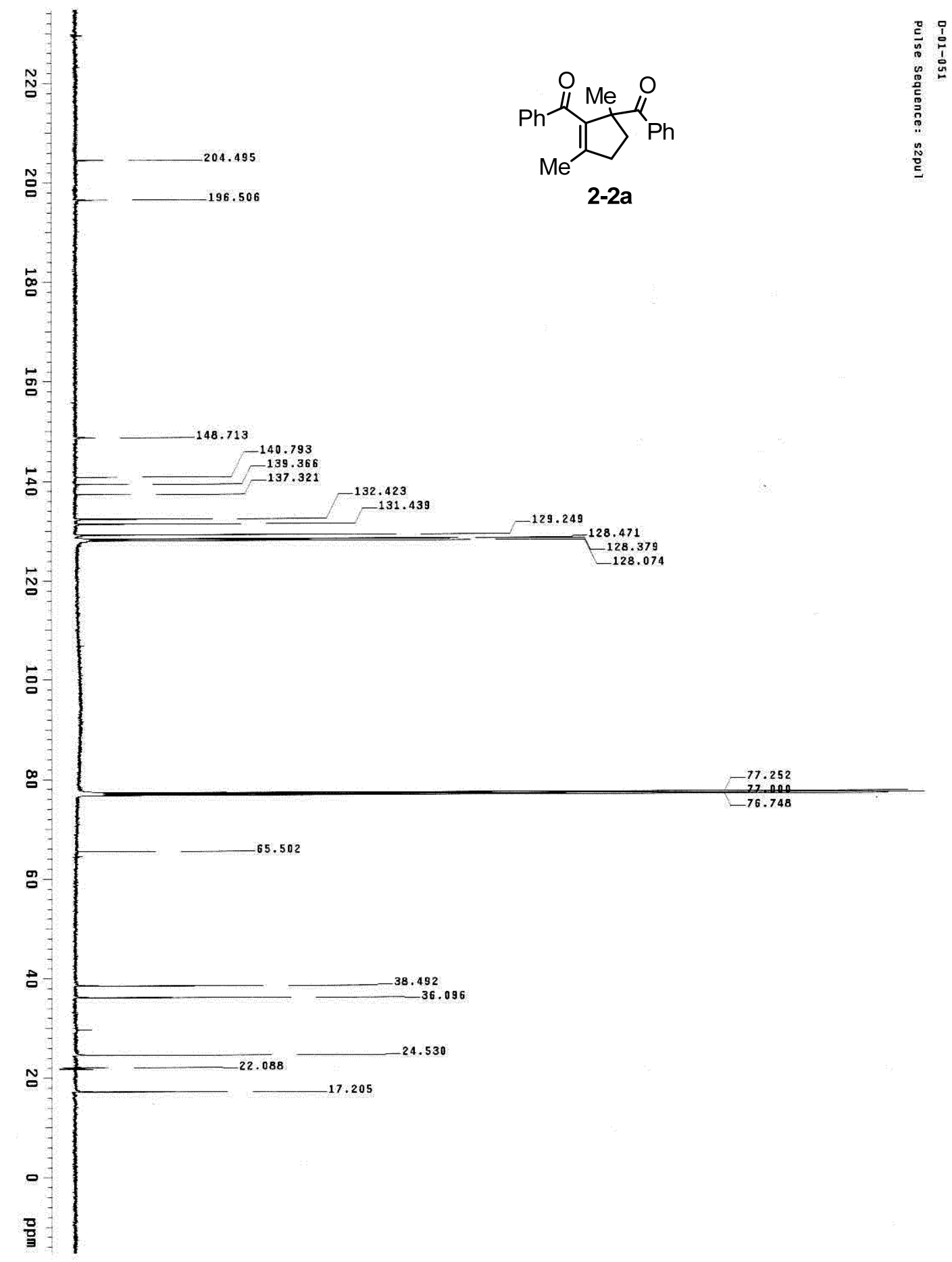




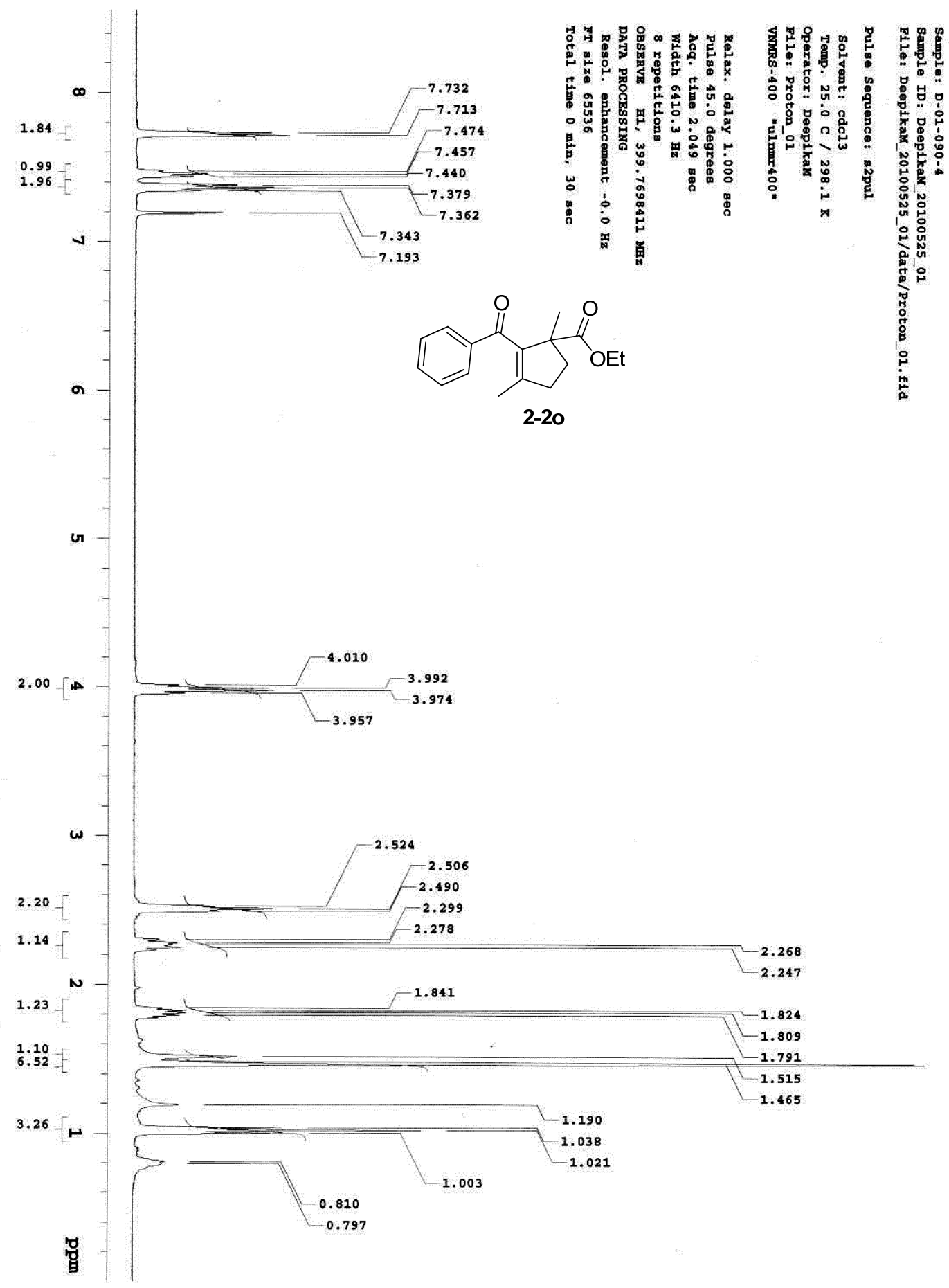



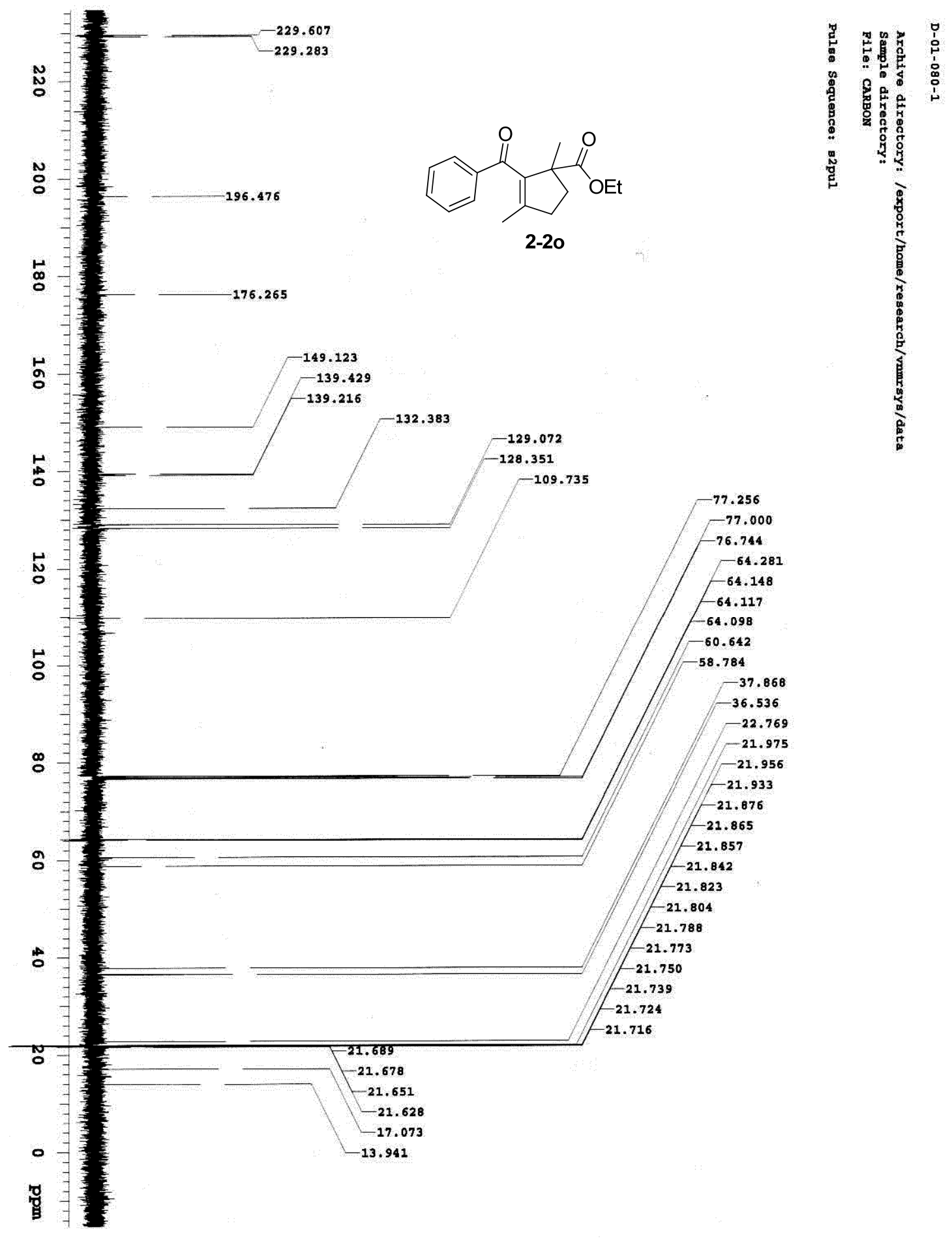


\section{CHAPTER 3. TANDEM MICHAEL ADDITION/ALDOL REACTIONS OF ALLENIC KETONES}

\subsection{Background}

In Chapter 2 the reactivity of 2-alkynyl-1,5-dicarbonyl compounds was explored. This work led to the construction of highly substituted five-membered rings bearing a quaternary carbon tethered to a carbonyl group. However, the synthesis of these versatile 2- alkynyl-1,5-dicarbonyl substrates is not trivial. A very practical method to synthesize these substrates was discoved while investigating the reactivity of alkynylenolates and other extended enolates. Because of the importance of 2-alkynyl-1,5-dicarbonyl as substrates, it was decided to describe the extensive details of their reaction scope and our new synthesis of these molecules in this chapter. Since Michael additions and aldol reactions are common synthetic tools for the construction of complex organic molecules, their enolate chemistry continues to enrich the arsenal of organic synthetic chemistry. ${ }^{47,50-}$ 52,54,56,177-182 The reactivity of alkynylenolates-an alkynyl-substituted enolate generated from allenoates or propargylic esters under basic conditions-makes them ideal nucleophiles in reactions such as aldol condensation, Michael addition, alkylation and halogenation (Scheme 25). ${ }^{82-87}$

To probe the reactivity of alkynylenolates, we speculated that the Michael addition of allenic ketones to vinyl ketones could yield 2-alkynyl 1,5-diketones, triggering an 
intramolecular aldol reaction leading to the formation of hydroxycyclohexanones or cyclohexenones in tandem fashion. ${ }^{183-189}$ Gratifyingly, we observed the allenic ketones underwent tandem Michael addition/aldol reaction with alkyl vinyl ketones to form 2-alkynyl 1,5-diketones, 4-alkynyl-3-hydroxycyclohexanones and 4-alkynyl cyclohexenones. Although 1,5-diketones, hydroxycyclohexanones and cyclohexenones have often been employed in organic synthesis and found as substructures in bioactive molecules or natural products, ${ }^{190-196}$ the synthetically more interesting 2-alkynylsubstituted analogs have been rarely reported in the literature. ${ }^{197-199}$

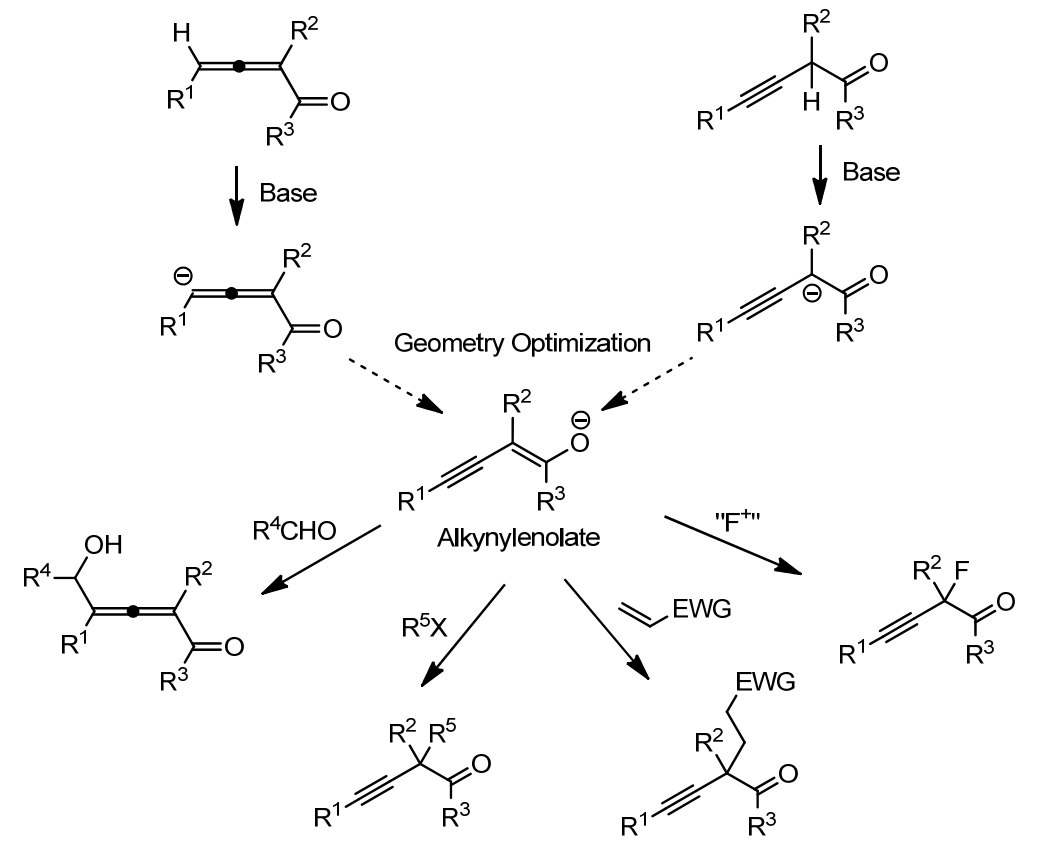

Scheme 25: Reactivity of alkynylenolates generated from allenoates or propargylic esters.

\subsection{Results and discussion}

To test this hypothesis, we first examined the reactivity of 2-methyl-1,4-diphenylbuta2,3-dien-1-one 3-1a and methyl vinyl ketone 3-2a (MVK) as model substrates. We investigated the formation of the products $\mathbf{3 - 3} \mathbf{- 3} \mathbf{- 3 - 5 a}$ as outlined in Table 2. As expected, 2-alkynyl 1,5-diketone 3-3a was obtained in good yield (73 \%) when tetrabutyl 
ammonium fluoride (TBAF) was used as the base (Table 2, entry 1) ${ }^{200-208}$ When potassium carbonate was employed as the base, only trace amounts of 3-3a was found and most of the starting material remained unreacted (Table 2, entry 2). Phase-transfer catalysis helped the reaction, as shown by the fact that 3-3a was obtained in moderate yield when tetrabutylammonium bromide (TBAB) was employed in the reaction (Table 2, entry 3). ${ }^{209-211}$ Also, under these weakly basic conditions, the tandem Michael addition/aldol condensation product 3-5a was isolated at higher temperature (Table 2, entry 4). When a strong base such as potassium hydroxide, lithium hydroxide, sodium hydroxide or cesium hydroxide was used in the reaction, compound 3-5a was obtained as the only product in most cases (Table 2, entries 5-9). Calcium hydroxide could not promote the reaction, possibly because of its poor solubility (Table 2, entry 10). Although potassium hydroxide could promote the reaction; due to its high hydrophilicity and lack of dispersion, it caused the reaction mixture to become dark quickly. We observed that when potassium carbonate was used as a co-promoter, the reaction became gentler and higher yield of product 3-5a was obtained (Table 2, entry 12). Product 3-5a was thought to arise from the dehydration of $\mathbf{3 - 4 a}$, prompting us to postulate that the dehydration would not take place at low temperature. Indeed, 3-4a was isolated as the major product in good yield when the reaction was conducted at $-40{ }^{\circ} \mathrm{C}$ (Table 2, entry 13). Other phase-transfer catalysts such as benzyltriethylammonium chloride $\left(\mathrm{BnNEt}_{3} \mathrm{Cl}\right)$ and tetrabutylammonium hydrogen sulfate $\left(\mathrm{Bu}_{4} \mathrm{NHSO}_{4}\right)$ were also investigated in the reaction, and similar results were obtained (Table 2, entries 14-15). The solvent effect was also studied and toluene was found to be the best solvent for the generation of product 35a (Table 2, entries 12 and 16-21). 

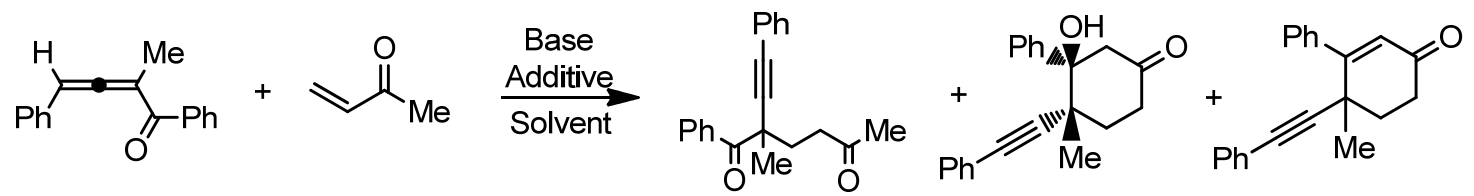

\begin{tabular}{|c|c|c|c|c|c|c|c|}
\hline \multirow{2}{*}{ Entry } & \multirow{2}{*}{ Base } & \multirow{2}{*}{ Additive } & \multirow{2}{*}{ Solvent } & \multirow{2}{*}{ Temperature } & \multicolumn{3}{|c|}{ yield(\% $)^{[\mathrm{b}]}$} \\
\hline & & & & & $3-3 a$ & $3-4 a$ & $3-5 a$ \\
\hline 1 & TBAF & none & THF & RT & 73 & 0 & 0 \\
\hline 2 & $\mathrm{~K}_{2} \mathrm{CO}_{3}$ & none & Toluene & RT & trace & 0 & 0 \\
\hline 3 & $\mathrm{~K}_{2} \mathrm{CO}_{3}$ & TBAB & Toluene & RT & 48 & 0 & 0 \\
\hline 4 & $\mathrm{~K}_{2} \mathrm{CO}_{3}$ & TBAB & Toluene & $80^{\circ} \mathrm{C}$ & 22 & 0 & 16 \\
\hline 5 & $\mathrm{KOH}$ & none & Toluene & RT & 0 & 0 & 36 \\
\hline 6 & $\mathrm{KOH}$ & TBAB & Toluene & RT & 0 & 0 & 54 \\
\hline 7 & $\mathrm{LiOH}$ & TBAB & Toluene & RT & 0 & 16 & 32 \\
\hline 8 & $\mathrm{NaOH}$ & TBAB & Toluene & RT & 0 & 0 & 42 \\
\hline 9 & $\mathrm{CsOH} \cdot \mathrm{H}_{2} \mathrm{O}$ & TBAB & Toluene & RT & 0 & 0 & 54 \\
\hline 10 & $\mathrm{Ca}(\mathrm{OH})_{2}$ & TBAB & Toluene & RT & 0 & 0 & 0 \\
\hline 11 & $\mathrm{Ca}(\mathrm{OH})_{2} / \mathrm{K}_{2} \mathrm{CO}_{3}$ & TBAB & Toluene & RT & 0 & 24 & 26 \\
\hline 12 & $\mathrm{KOH} / \mathrm{K}_{2} \mathrm{CO}_{3}$ & TBAB & Toluene & RT & 0 & 0 & 71 \\
\hline $13^{c}$ & $\mathrm{KOH} / \mathrm{K}_{2} \mathrm{CO}_{3}$ & TBAB & Toluene & $-40^{\circ} \mathrm{C}$ & trace & 83 & 0 \\
\hline 14 & $\mathrm{KOH} / \mathrm{K}_{2} \mathrm{CO}_{3}$ & $\mathrm{BnNEt}_{3} \mathrm{Cl}$ & Toluene & RT & 0 & 0 & 55 \\
\hline 15 & $\mathrm{KOH} / \mathrm{K}_{2} \mathrm{CO}_{3}$ & $\mathrm{Bu}_{4} \mathrm{NHSO}_{4}$ & Toluene & RT & 0 & 0 & 58 \\
\hline 16 & $\mathrm{KOH} / \mathrm{K}_{2} \mathrm{CO}_{3}$ & TBAB & $\mathrm{CH}_{2} \mathrm{Cl}_{2}$ & RT & 0 & 16 & 35 \\
\hline 17 & $\mathrm{KOH} / \mathrm{K}_{2} \mathrm{CO}_{3}$ & TBAB & DMF & RT & 0 & 0 & 26 \\
\hline 18 & $\mathrm{KOH} / \mathrm{K}_{2} \mathrm{CO}_{3}$ & TBAB & THF & RT & 0 & 0 & 49 \\
\hline 19 & $\mathrm{KOH} / \mathrm{K}_{2} \mathrm{CO}_{3}$ & TBAB & Dioxane & RT & 0 & 14 & 44 \\
\hline 20 & $\mathrm{KOH} / \mathrm{K}_{2} \mathrm{CO}_{3}$ & TBAB & $\mathrm{Et}_{2} \mathrm{O}$ & RT & 0 & 20 & 32 \\
\hline 21 & $\mathrm{KOH} / \mathrm{K}_{2} \mathrm{CO}_{3}$ & TBAB & $\mathrm{CH}_{3} \mathrm{CN}$ & RT & 0 & 17 & 25 \\
\hline
\end{tabular}

Table 2. Base-mediated reactions of allenic ketone 3-1a with methyl vinyl ketone. ${ }^{\text {a }}$ 


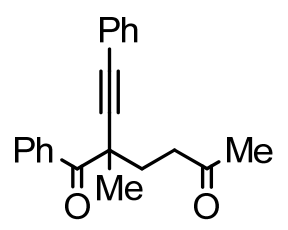

3-3a

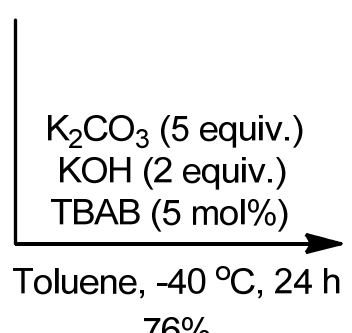

$76 \%$

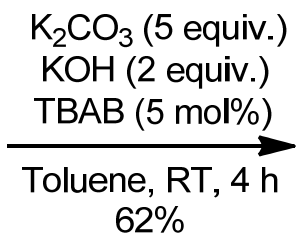

$62 \%$

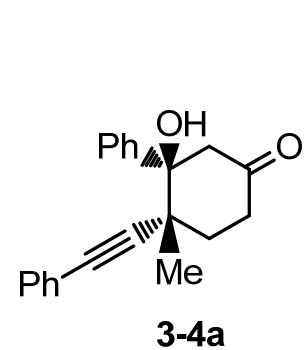

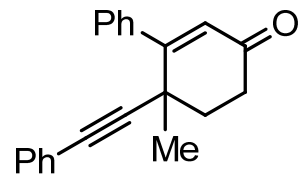

3-5a

Scheme 26: Control transformations between compounds 3-3a, 3-4a and 3-5a.

To demonstrate the transformations between 3-3a, 3-4a and 3-5a, we performed control reactions under the optimized conditions and the results are summarized in Scheme 26. It was found that 3-3a could transform into 3-4a under basic conditions at low temperature. Both compounds 3-3a and 3-4a could transform into 3-5a under the same conditions at room temperature. With the optimum conditions in hand, we proceeded to study the scope and limitations of these reactions. First, we investigated the TBAF-mediated Michael addition of allenic ketones 3-1 with vinyl ketones 3-2 to obtain 2-alkynyl 1,5diketones 3-3 (Table 3). These reactions proceeded smoothly for both aromatic and aliphatic substrates, furnishing 3-3 in moderate to good yields. In next step, we carried out the tandem Michael addition/aldol reaction of allenic ketones 3-1 with vinyl ketones 3-2 at low temperature $\left(-40^{\circ} \mathrm{C}\right)$, with the corresponding products $\mathbf{3 - 4}$ obtained in moderate to good yields (Table 4). This reaction was slower under the utilized conditions, and prolonged reaction times were needed. Higher yields were obtained for aromatic substrates, which may be due to the higher stability of the aromatic system. Only one diastereoisomer of the product was obtained, even when ethyl vinyl ketone $\mathbf{3 - 2 b}$ was used as the Michael acceptor, as determined by the ${ }^{1} \mathrm{H}$ and ${ }^{13} \mathrm{C}$ NMR spectroscopy. 
Table 3: TBAF-mediated Michael addition of allenic ketones 3-1 to vinyl ketones 3-2. ${ }^{\text {[a] }}$

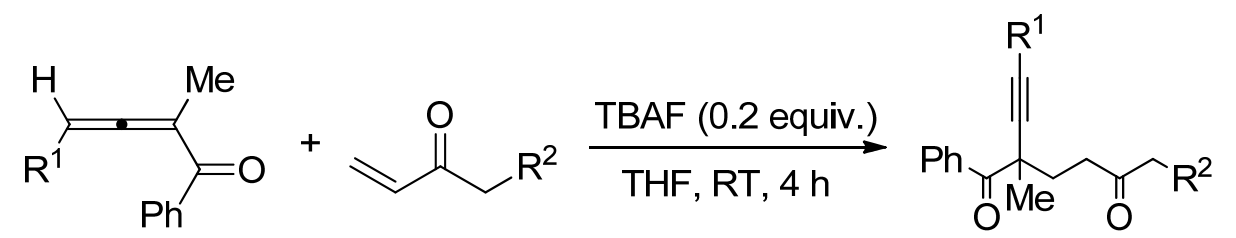

\begin{tabular}{|c|c|c|c|}
\hline Entry & $\mathrm{R}^{1}$ & $\mathrm{R}^{2}$ & Yield $(\%)^{[\mathrm{b}]}$ \\
\hline 1 & $\mathrm{C}_{6} \mathrm{H}_{5}(3-1 \mathrm{a})$ & $H(3-2 a)$ & $3-3 a, 73$ \\
\hline 2 & $p-\mathrm{CH}_{3} \mathrm{OC}_{6} \mathrm{H}_{4}(3-1 b)$ & $H(3-2 a)$ & 3-3b, 76 \\
\hline 3 & $p-\mathrm{ClC}_{6} \mathrm{H}_{4}(3-1 \mathrm{c})$ & $H(3-2 a)$ & $3-3 c, 71$ \\
\hline 4 & $n-\mathrm{C}_{6} \mathrm{H}_{13}(3-1 d)$ & $H(3-2 a)$ & 3-3d, 37 \\
\hline 5 & $i-\operatorname{Pr}(3-1 e)$ & $H(3-2 a)$ & $3-3 e, 63$ \\
\hline 6 & $t$-Bu (3-1f) & $H(3-2 a)$ & 3-3f, 65 \\
\hline 7 & $\mathrm{Bn}(3-1 \mathrm{~g})$ & $H(3-2 a)$ & $3-3 g, 72$ \\
\hline 8 & $\mathrm{CypCH}_{2}(3-1 h)$ & $H(3-2 a)$ & $3-3 h, 41$ \\
\hline 9 & $\mathrm{CH}_{3}(3-1 \mathrm{i})$ & $H(3-2 a)$ & 3-3i, 69 \\
\hline 10 & $\mathrm{C}_{6} \mathrm{H}_{5}(3-1 \mathrm{a})$ & $\mathrm{CH}_{3}(3-2 b)$ & 3-3j, 73 \\
\hline 11 & $p-\mathrm{CH}_{3} \mathrm{OC}_{6} \mathrm{H}_{4}(3-1 b)$ & $\mathrm{CH}_{3}(3-2 b)$ & 3-3k, 57 \\
\hline 12 & $p-\mathrm{ClC}_{6} \mathrm{H}_{4}(3-1 \mathrm{c})$ & $\mathrm{CH}_{3}(3-2 b)$ & 3-3I, 54 \\
\hline
\end{tabular}

[a] General reaction conditions: allenic ketones 3-1 $(0.4 \mathrm{mmol})$, vinyl ketones 3-2 $(0.6 \mathrm{mmol})$, TBAF $(0.2$ equiv.), THF $(2.0 \mathrm{~mL})$. [b] Isolated yields. 
Table 4. Tandem Michael addition/aldol reaction of allenic ketones 3-1 with vinyl ketones 3-2 at low temperature. ${ }^{[a]}$

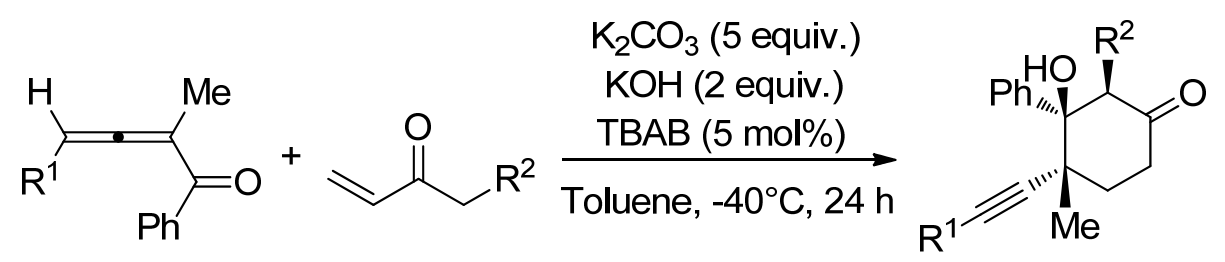

\begin{tabular}{|c|c|c|c|}
\hline $3-1$ & $3-2$ & & $3-4$ \\
\hline Entry & $\mathrm{R}^{1}$ & $\mathrm{R}^{2}$ & Yield(\%) $)^{[\mathrm{b}]}$ \\
\hline 1 & $\mathrm{C}_{6} \mathrm{H}_{5}(3-1 \mathrm{a})$ & $H(3-2 a)$ & $3-4 a, 83$ \\
\hline 2 & $p-\mathrm{CH}_{3} \mathrm{OC}_{6} \mathrm{H}_{4}(\mathbf{3 - 1 b})$ & $H(3-2 a)$ & $3-4 b, 52$ \\
\hline 3 & $p-\mathrm{ClC}_{6} \mathrm{H}_{4}(3-1 \mathrm{c})$ & $H(3-2 a)$ & $3-4 c, 49$ \\
\hline 4 & $n-\mathrm{C}_{6} \mathrm{H}_{13}(\mathbf{3 - 1 d})$ & $H(3-2 a)$ & $3-4 d, 32$ \\
\hline 5 & $i-\operatorname{Pr}(3-1 e)$ & $H(3-2 a)$ & $3-4 e, 32$ \\
\hline 6 & $t$-Bu (3-1f) & $H(3-2 a)$ & $3-4 f, 39$ \\
\hline 7 & $\mathrm{CypCH}_{2}(3-1 h)$ & $H(3-2 a)$ & $3-4 g, 28$ \\
\hline 8 & $\mathrm{CH}_{3}(3-1 \mathrm{i})$ & $H(3-2 a)$ & $3-4 h, 53$ \\
\hline 9 & $\mathrm{C}_{6} \mathrm{H}_{5}(3-1 \mathrm{a})$ & $\mathrm{CH}_{3}(\mathbf{3}-\mathbf{2 b})$ & $3-4 i, 59$ \\
\hline 10 & $p-\mathrm{CH}_{3} \mathrm{OC}_{6} \mathrm{H}_{4}(\mathbf{3}-1 \mathbf{b})$ & $\mathrm{CH}_{3}(\mathbf{3 - 2 b})$ & $3-4 \mathbf{j}, 41$ \\
\hline 11 & $n-\mathrm{C}_{6} \mathrm{H}_{13}(3-1 \mathrm{~d})$ & $\mathrm{CH}_{3}(3-2 b)$ & $3-4 k, 35$ \\
\hline
\end{tabular}

[a] General reaction conditions: allenic ketones 3-1 (0.4 mmol), vinyl ketones 3-2 (0.6 mmol), $\mathrm{KOH}$ (2 equiv.), $\mathrm{K}_{2} \mathrm{CO}_{3}$ (5 equiv.), TBAB (5 mol\%), Toluene $(2.0 \mathrm{~mL})$. [b] Isolated yields. 


\subsubsection{Conformation analysis of transition states and NOESY effect of compound 3-4h}

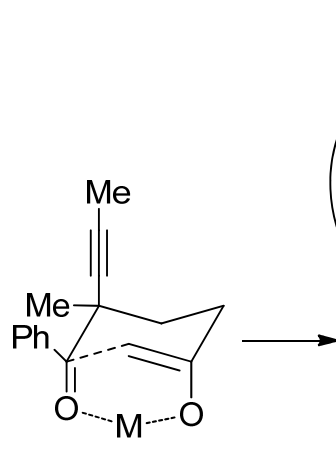

TS-1

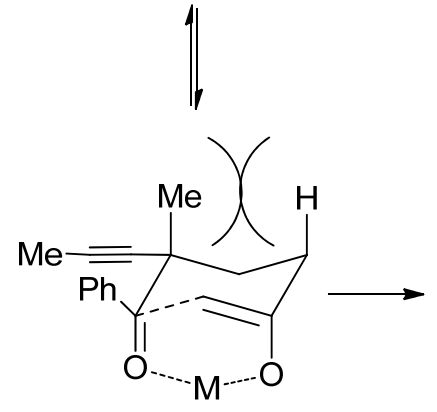

TS-2

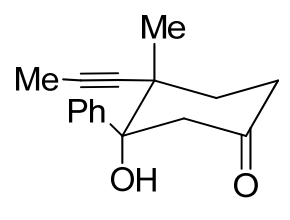

cis-3-4h

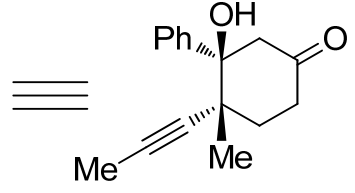

trans-3-4h

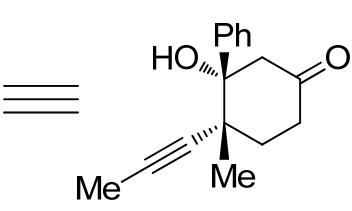

not found

Scheme 27. Conformation analysis of transition states and NOESY effect of compound 3-4h.

The configuration of substrate 3-4 was established by the conformational analysis of the transition states and was confirmed by NOESY studies of compounds 3-4h and 34i (Scheme 27 and Scheme 28). When methyl vinyl ketone (MVK) was used in the reaction, transition state TS-1 was kinetically more favorable than TS-2 because there is a 1,3-diaxial interaction between the methyl group and the axial proton in TS-2, whereas the interaction is diminished in TS-1, where the alkynyl moiety is axial. Thus, trans-34h was obtained as product through TS-1 instead of cis-3-4h through TS-2 and this configuration was also supported by NOESY experiment on the product. Moreover, when ethyl vinyl ketone (EVK) was used as the Michael acceptor in the reaction, the more favorable transition state was also the one in which the alkynyl group is axial and the 
methyl $^{(\mathrm{b})}$ group is equatorial. On the other hand, the Z-enolate in TS-3 is also less sterically hindered than the E-enolate in TS-4. Therefore, TS-3 is more favorable than TS-4, and cis,trans-3-4i was obtained from the reaction instead of trans,trans-3-4i. The configuration of 3-4i was also confirmed by its NOESY effect, where spatial proximity exists between the two axial protons, between the methyl ${ }^{(a)}$ group and the hydroxy group, and between the proton $^{(a)}$ and the phenyl group.

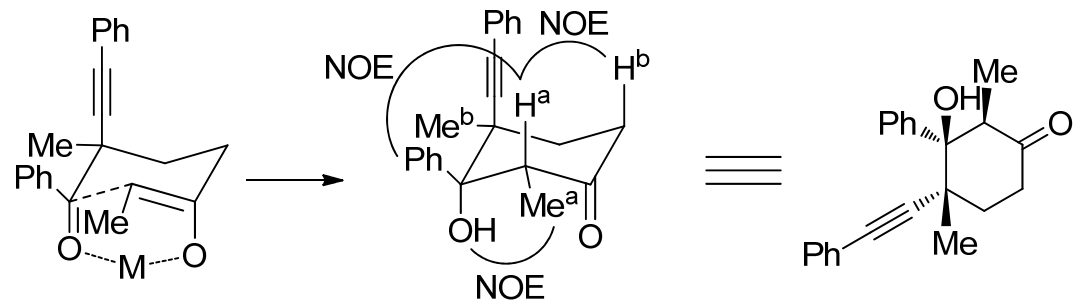

TS-3

cis, trans-3-4i

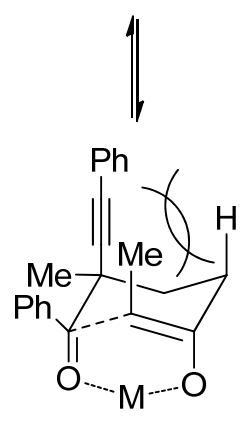

TS-4

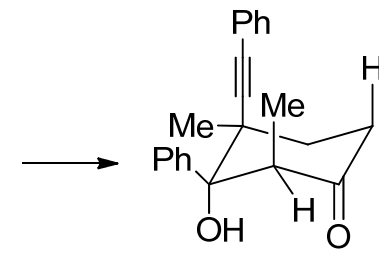

trans, trans-3-4i

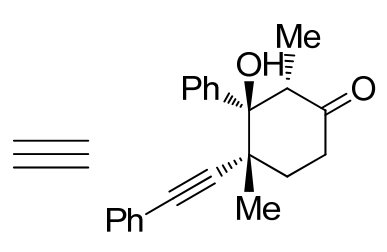

not found

Scheme 28: Conformation analysis of transition states and NOESY effect of compound 3-4i.

The tandem Michael addition/aldol condensation of allenic ketones 3-1 with vinyl ketones 3-2 at room temperature was also explored and the corresponding 4alkynylcyclohexenones 3-5 were obtained as the products in moderate to good yields (Table 5). These reactions also proceeded smoothly and better results were obtained with aromatic substrates. 
Table 5: Tandem Michael addition/aldol condensation of allenic ketones 3-1 with vinyl ketones 3-2 at room temperature. ${ }^{[a]}$

\begin{tabular}{|c|c|c|c|}
\hline$\overbrace{\mathrm{Ph}}^{\mathrm{H}}=\mathrm{Me}$ & $\stackrel{\mathrm{O}}{\mathrm{R}_{2}}$ & $\begin{array}{c}\mathrm{K}_{2} \mathrm{CO}_{3} \text { (5 equiv.) } \\
\mathrm{KOH}(2 \text { equiv. }) \\
\text { TBAB (5 mol\% }) \\
\text { Toluene, RT, } 4 \mathrm{~h}\end{array}$ & (N) \\
\hline Entry & $\mathrm{R}_{1}$ & $\mathrm{R}_{2}$ & Yield(\%) $)^{[b]}$ \\
\hline 1 & $\mathrm{C}_{6} \mathrm{H}_{5}(3-1 \mathrm{a})$ & $H(3-2 a)$ & $3-5 a, 71$ \\
\hline 2 & $\mathrm{p}-\mathrm{CH}_{3} \mathrm{OC}_{6} \mathrm{H}_{4}(3-1 b)$ & $H(3-2 a)$ & $3-5 b, 53$ \\
\hline 3 & $\mathrm{p}-\mathrm{ClC}_{6} \mathrm{H}_{4}(3-1 \mathrm{c})$ & $H(3-2 a)$ & $3-5 c, 51$ \\
\hline 4 & $n-\mathrm{C}_{6} \mathrm{H}_{13}(3-1 d)$ & $H(3-2 a)$ & $3-5 d, 47$ \\
\hline 5 & $i-\operatorname{Pr}(3-1 e)$ & $H(3-2 a)$ & $3-5 e, 33$ \\
\hline 6 & $t$-Bu (3-1f) & $H(3-2 a)$ & $3-5 f, 45$ \\
\hline 7 & $\mathrm{CypCH}_{2}(\mathbf{3 - 1 h})$ & $H(3-2 a)$ & $3-5 g, 31$ \\
\hline 8 & $\mathrm{CH}_{3}(3-1 \mathrm{i})$ & $H(3-2 a)$ & 3-5h, 49 \\
\hline 9 & $\mathrm{C}_{6} \mathrm{H}_{5}(3-1 \mathrm{a})$ & $\mathrm{CH}_{3}(3-2 b)$ & $3-5 i, 59$ \\
\hline 10 & $p-\mathrm{CH}_{3} \mathrm{OC}_{6} \mathrm{H}_{4}(3-1 \mathbf{b})$ & $\mathrm{CH}_{3}(3-2 b)$ & 3-5j, 51 \\
\hline 11 & $p-\mathrm{ClC}_{6} \mathrm{H}_{4}(3-1 \mathrm{c})$ & $\mathrm{CH}_{3}(3-2 b)$ & $3-5 k, 42$ \\
\hline 12 & $n-\mathrm{C}_{6} \mathrm{H}_{13}(3-1 d)$ & $\mathrm{CH}_{3}(3-2 b)$ & $3-5 I, 39$ \\
\hline
\end{tabular}

[a] General reaction conditions: allenic ketones 3-1 (0.4 mmol), vinyl ketones 3-2 (0.6 mmol), $\mathrm{KOH}(2$ equiv.), $\mathrm{K}_{2} \mathrm{CO}_{3}$ (5 equiv.), TBAB (5 mol\%), Toluene (2.0 mL). [b] Isolated yields.

\subsection{Summary}

In this chapter the reactivity and scope of alkynylenolate was investigated using allenic ketones and vinyl ketones as substrates and various alkynyl-substituted molecules were obtained. We developed a TBAF-mediated Michael addition reaction furnishing 2alkynyl 1,5-diketones and these substrates were readily employed as starting material for the construction of $\alpha, \beta$-unsaturated enones as described in Chapter 2. Moreover, under 
stronger basic conditions, a tandem Michael addition/aldol reaction of the two starting materials was reported and 4-alkynyl-3-hydroxycyclohexanones were isolated at low temperature, whereas 4-alkynylcyclohexenones were obtained at room temperature. The conformational analysis of the reaction transition states provided a reasonable explanation for the formation of a single diastereoisomer of 3-4. The work described in this chapter was published in Eur. J. Org. Chem. 2010, 35, 6855-6862.

\subsection{Experimental}

\section{General}

${ }^{1} \mathrm{H}$ and ${ }^{13} \mathrm{C}$ NMR spectra were recorded at 500, 126 (or 400 and 101) $\mathrm{MHz}$ respectively, using $\mathrm{CDCl}_{3}$ as a solvent. The chemical shifts are reported in $\delta(\mathrm{ppm})$ values relative to $\mathrm{CHCl}_{3}\left(\delta 7.26 \mathrm{ppm}\right.$ for ${ }^{1} \mathrm{H}$ NMR and $\delta 77.0 \mathrm{ppm}$ for ${ }^{13} \mathrm{C}$ NMR), multiplicities are indicated by s (singlet), d (doublet), t (triplet), q (quartet), $\mathrm{p}$ (pentet), h (hextet), $\mathrm{m}$ (multiplet) and br (broad). Coupling constants, $J$, are reported in Hertz. Coupling constants are reported in hertz $(\mathrm{Hz})$. All air and/or moisture sensitive reactions were carried out under argon atmosphere. Solvents (tetrahydrofuran, ether, dichloromethane and toluene) were chemically dried using a commercial solvent purification system. All other reagents and solvents were employed without further purification. The products were purified using a Biotage flash ${ }^{+}$system or Chromatotron apparatus or a regular glass column. TLC was developed on Merck silica gel 60 F254 aluminum sheets. Elemental analysis was performed at Atlantic Microlabs Inc., Norcross, Georgia 30091. High resolution ESI-MS were obtained using a MS-FTICR-MS ${ }^{\mathrm{n}}$ system (LTQ FT, Thermo 
Electron Corp.) at the CREAM Mass Spectrometry Facility, University of Louisville, Kentucky.

General procedure for the synthesis of 2-alkynyl 1,5-diketones 3-3:

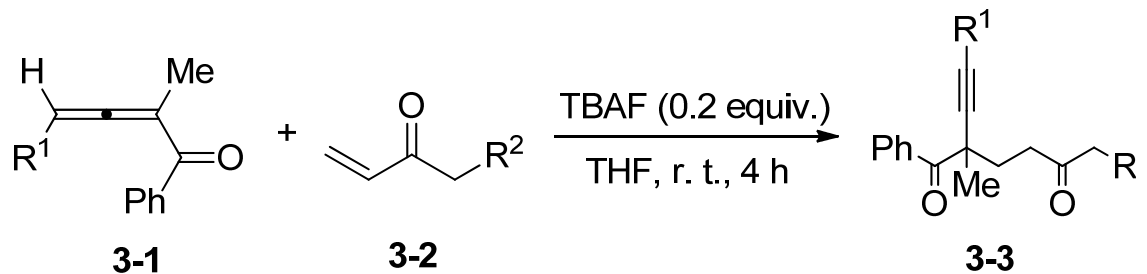

To a solution of allenic ketone 3-1 $(0.50 \mathrm{mmol})$ in THF $(2.0 \mathrm{~mL})$ was added methyl vinyl ketone (MVK) 3-2 $(0.60 \mathrm{mmol})$ and TBAF $(0.1 \mathrm{~mL}, 1 \mathrm{~m}$ in THF, $0.10 \mathrm{mmol})$. The mixture was stirred for $4 \mathrm{~h}$ at room temperature. The reaction mixture was quenched with water and extracted with diethyl ether. The solvent of the organic layer was removed under reduced pressure and the residue was subjected to a flash column chromatography (eluent: ethyl acetate $/ n$-hexane $=1: 10$ ) to give product 3-3 as a colorless oil.

\section{General procedure for the synthesis of 4-alkynyl- 3hydroxycyclohexanones 3-4:}

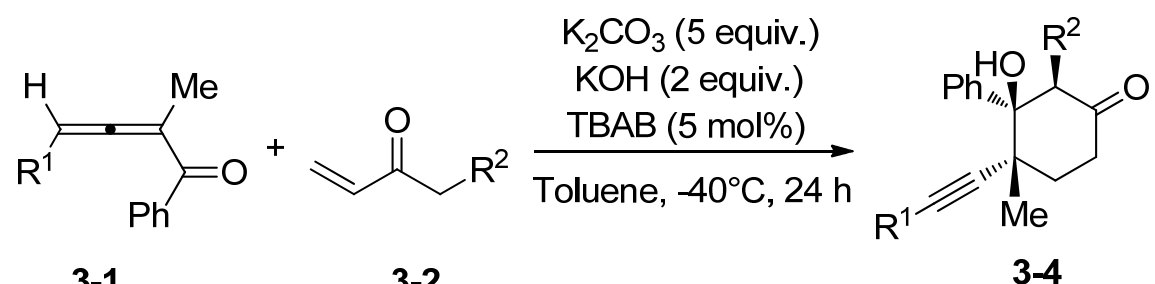

3-1

3-2

To a solution of allenic ketone 3-1 $(0.50 \mathrm{mmol})$ in toluene $(2.0 \mathrm{~mL})$ was added methyl vinyl ketone (MVK) 3-2 $(0.60 \mathrm{mmol})$ and TBAB $(5 \mathrm{~mol} \%)$. The mixture was cooled to $40{ }^{\circ} \mathrm{C}$ followed by the addition of potassium carbonate $(2.5 \mathrm{mmol})$ and potassium hydroxide $(1 \mathrm{mmol})$. The mixture was stirred at $-40{ }^{\circ} \mathrm{C}$ for $24 \mathrm{~h}$. The reaction mixture 
was quenched with water and extracted with diethyl ether. The solvent of the organic layer was removed under reduced pressure and the residue was subjected to a flash column chromatography (eluent: ethyl acetate $/ n$-hexane $=1: 10$ ) to give product 3-4 as a colorless oil.

\section{General procedure for the synthesis of 4-alkynylcyclohexenones 3-5:}

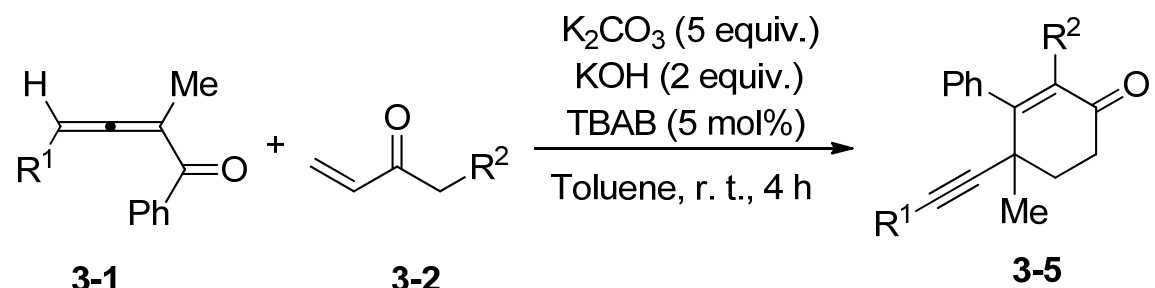

To a solution of allenic ketone 3-1 $(0.50 \mathrm{mmol})$ in toluene $(2.0 \mathrm{~mL})$ was added methyl vinyl ketone (MVK) 3-2 (0.60 mmol), TBAB (5 mol-\%), potassium carbonate $(2.5 \mathrm{mmol})$ and potassium hydroxide $(1 \mathrm{mmol})$. The mixture was stirred at room temperature for $4 \mathrm{~h}$. The reaction mixture was quenched with water and extracted with diethyl ether. The solvent of the organic layer was removed under reduced pressure and the residue was subjected to a flash column chromatography (eluent: ethyl acetate $/ n$-hexane $=1: 10$ ) to give product 3-5 as a colorless oil.

\section{Spectroscopic data of compounds 3-3.}

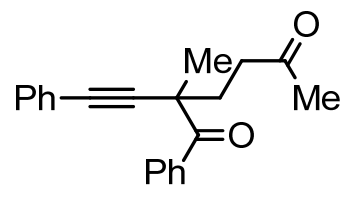

3-3a

Data for 3-3a: A colorless oil. IR (neat): $\bar{v}=2982,1716,1682,1596,1490,1363,1168$, $965 \mathrm{~cm}^{-1} .{ }^{1} \mathrm{H}$ NMR $\left(\mathrm{CDCl}_{3}, 500 \mathrm{MHz}\right): \delta=1.58(\mathrm{~s}, 3 \mathrm{H}), 1.95-2.01(\mathrm{~m}, 1 \mathrm{H}), 2.08(\mathrm{~s}, 3$ 
H), 2.34-2.43 (m, $1 \mathrm{H}), 2.52-2.58(\mathrm{~m}, 1 \mathrm{H}), 2.63-2.69(\mathrm{~m}, 1 \mathrm{H}), 7.18-7.27(\mathrm{~m}, 5 \mathrm{H})$, 7.35-7.38 (t, $J=7.5 \mathrm{~Hz}, 2 \mathrm{H}), 7.45-7.48(\mathrm{t}, J=7.5 \mathrm{~Hz}, 1 \mathrm{H}), 8.23-8.25(\mathrm{~d}, J=8.0 \mathrm{~Hz}, 2$ H) ppm. ${ }^{13} \mathrm{C}$ NMR ( $\left.\mathrm{CDCl}_{3}, 126 \mathrm{MHz}\right): \delta=26.6,30.0,33.6,39.7,45.9,86.7,91.5,122.8$, 128.0, 128.3, 129.7, 131.3, 132.7, 135.3, 198.8, 208.0 ppm. $\mathrm{C}_{21} \mathrm{H}_{20} \mathrm{O}_{2}$ (304.14): calcd. C 82.86, H 6.62, found C 82.75, H 6.62 .

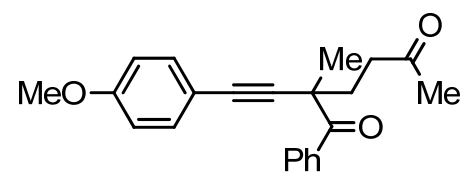

3-3b

Data for 3-3b: Colorless oil. IR (neat): $\bar{v}=2959,2933,1716,1681,1606,1509,1446$, 1289, 1172, 1031, 966, $690 \mathrm{~cm}^{-1} .{ }^{1} \mathrm{H}$ NMR $\left(\mathrm{CDCl}_{3}, 500 \mathrm{MHz}\right): \delta=1.65(\mathrm{~s}, 3 \mathrm{H}), 2.02-$ $2.08(\mathrm{~m}, 1 \mathrm{H}), 2.17$ (s, $3 \mathrm{H}), 2.44-2.49(\mathrm{~m}, 1 \mathrm{H}), 2.61-2.66(\mathrm{~m}, 1 \mathrm{H}), 2.66-2.78(\mathrm{~m}, 1 \mathrm{H})$, 3.79 (s, 3 H), 6.81-6.84 (m, 2 H), 7.27-7.30 (m, 2 H), 7.43-7.46 (m, 2 H), 7.52-7.55 (m, $1 \mathrm{H}), 8.32-8.33(\mathrm{~m}, 2 \mathrm{H}) \mathrm{ppm} .{ }^{13} \mathrm{C} \mathrm{NMR}\left(\mathrm{CDCl}_{3}, 126 \mathrm{MHz}\right): \delta=26.9,30.3,33.9,40.0$, $46.1,55.5,86.8,90.2,114.2,115.2,128.3,130.0,132.9,133.0,135.6,159.8,199.3,208.3$ ppm.

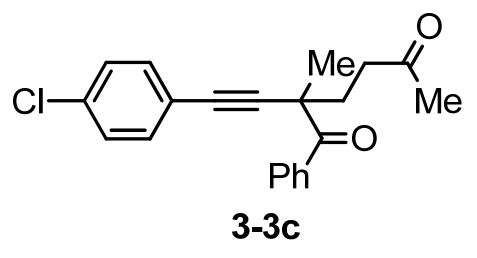

Data for 3-3c: A colorless oil. IR (neat): $\bar{v}=2932,2870,1717,1683,1489,1239,1090$, 966, $715 \mathrm{~cm}^{-1} .{ }^{1} \mathrm{H} \mathrm{NMR}\left(\mathrm{CDCl}_{3}, 500 \mathrm{MHz}\right): \delta=1.64(\mathrm{~s}, 3 \mathrm{H}), 2.01-2.09(\mathrm{~m}, 1 \mathrm{H}), 2.08$ (s, $3 \mathrm{H}), 2.42-2.49(\mathrm{~m}, 1 \mathrm{H}), 2.54-2.61(\mathrm{~m}, 1 \mathrm{H}), 2.67-2.71(\mathrm{~m}, 1 \mathrm{H}), 7.24-7.25(\mathrm{~d}, J=$ $2.8 \mathrm{~Hz}, 4 \mathrm{H}), 7.43-7.45$ (t, $J=6.8 \mathrm{~Hz}, 2 \mathrm{H}), 7.51-7.55(\mathrm{t}, J=6.5 \mathrm{~Hz}, 1 \mathrm{H}), 8.25-8.28$ (d, $J=8.0 \mathrm{~Hz}, 2 \mathrm{H}) \mathrm{ppm} .{ }^{13} \mathrm{C} \mathrm{NMR}\left(\mathrm{CDCl}_{3}, 126 \mathrm{MHz}\right): \delta=26.5,30.0,33.6,39.7,45.9$, 
85.6, 92.5, 121.3, 128.1, 128.7, 129.6, 132.5, 132.8, 134.4, 135.2, 198.6, 207.9 ppm. $\mathrm{C}_{21} \mathrm{H}_{19} \mathrm{ClO}_{2}$ (338.10): calcd. C 74.44, H 5.65, found C 74.36, H 5.68.

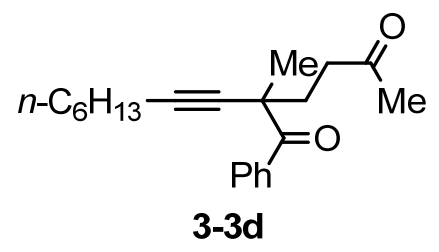

Data for 3-3d: Colorless oil. IR (neat): $\bar{v}=2931,2858,1717,1681,1441,1240,1176$, 967, $714 \mathrm{~cm}^{-1} .{ }^{1} \mathrm{H} \mathrm{NMR}\left(\mathrm{CDCl}_{3}, 500 \mathrm{MHz}\right): \delta=0.86-0.89(\mathrm{t}, J=7.0 \mathrm{~Hz}, 3 \mathrm{H}), 1.26-$ 1.47 (m, 9 H), 1.54 (s, 3 H), 1.91-1.95 (m, 1 H), 2.16-2.20 (m, 4 H), 2.31-2.37 (m, 1 H), 2.51-2.58 (m, $1 \mathrm{H}), 2.65-2.72(\mathrm{~m}, 1 \mathrm{H}), 7.41-7.44(\mathrm{t}, J=7.5 \mathrm{~Hz}, 2 \mathrm{H}), 7.52-7.54(\mathrm{t}, J=$ $7.0 \mathrm{~Hz}, 1 \mathrm{H}), 8.27-8.28(\mathrm{~d}, J=7.5 \mathrm{~Hz}, 2 \mathrm{H}) \mathrm{ppm} .{ }^{13} \mathrm{C} \mathrm{NMR}\left(\mathrm{CDCl}_{3}, 126 \mathrm{MHz}\right): \delta=14.2$, $19.1,22.8,27.1,28.7,30.2,31.5,34.0,40.1,45.6,65.2,82.4,87.4,128.1,130.1,132.7$, 135.6, 200.1, $208.5 \mathrm{ppm}$.

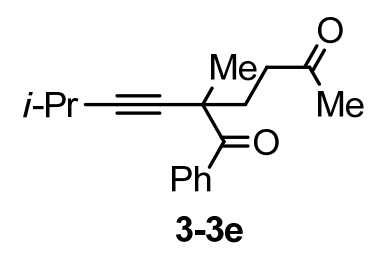

Data for 3-3e: Colorless oil. IR (neat): $\bar{v}=2929,2871,1715,1651,1555,1279,1178$, 965, 763, $694 \mathrm{~cm}^{-1} .{ }^{1} \mathrm{H} \mathrm{NMR}\left(\mathrm{CDCl}_{3}, 500 \mathrm{MHz}\right): \delta=1.11-1.12(\mathrm{~d}, J=7.0 \mathrm{~Hz}, 6 \mathrm{H}), 1.52$ (s, 3 H), 1.69-1.93 (m, 1 H), 2.16 (s, 3 H), 2.20-2.35 (m, 1 H), 2.51-2.56 (m, 2 H), 2.622.69 (m, $1 \mathrm{H}), 7.34-7.43$ (t, $J=8.0 \mathrm{~Hz}, 2 \mathrm{H}), 7.50-7.51(\mathrm{~m}, 1 \mathrm{H}), 8.26-8.28$ (dd, J = 7.5, $1.5 \mathrm{~Hz}, 2 \mathrm{H}) \mathrm{ppm} .{ }^{13} \mathrm{C} \mathrm{NMR}\left(\mathrm{CDCl}_{3}, 126 \mathrm{MHz}\right): \delta=20.6,22.7,26.7,29.9,33.6,39.7$, 45.2, 81.5, 92.4, 127.7, 129.8, 132.4, 135.4, 199.6, 208.2 ppm. $\mathrm{C}_{18} \mathrm{H}_{22} \mathrm{O}_{2}$ (270.16): calcd. C 79.96, H 8.20, found C 79.69, H 8.20. 


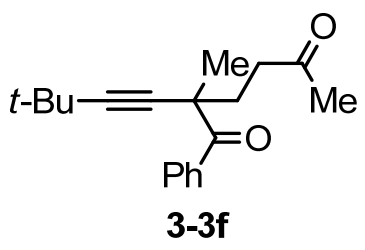

Data for 3-3f: Colorless oil. IR (neat): $\bar{v}=2967,2931,1717,1683,1596,1456,1172$, 966, $715 \mathrm{~cm}^{-1} .{ }^{1} \mathrm{H}$ NMR $\left(\mathrm{CDCl}_{3}, 500 \mathrm{MHz}\right): \delta=1.16(\mathrm{~s}, 9 \mathrm{H}), 1.51(\mathrm{~s}, 3 \mathrm{H}), 1.86-1.92$ (m, $1 \mathrm{H}), 2.15(\mathrm{~s}, 3 \mathrm{H}), 2.29-2.35(\mathrm{~m}, 1 \mathrm{H}), 2.47-2.55(\mathrm{~m}, 1 \mathrm{H}), 2.62-2.68(\mathrm{~m}, 1 \mathrm{H})$, 7.39-7.42 (t, $J=7.5 \mathrm{~Hz}, 2 \mathrm{H}), 7.49-7.52(\mathrm{t}, J=7.5 \mathrm{~Hz}, 1 \mathrm{H}), 8.26-8.27$ (d, $J=7.5 \mathrm{~Hz}, 2$ H) ppm. ${ }^{13} \mathrm{C} \mathrm{NMR}\left(\mathrm{CDCl}_{3}, 126 \mathrm{MHz}\right): \delta=26.8,27.5,29.9,30.7,33.6,39.7,45.1,80.8$, 95.2, 127.7, 129.8, 132.4, 135.4, 199.7, 208.2 ppm. $\mathrm{C}_{19} \mathrm{H}_{24} \mathrm{O}_{2}$ (284.17): calcd. C 80.24, H 8.51, found C 79.95, H 8.41.

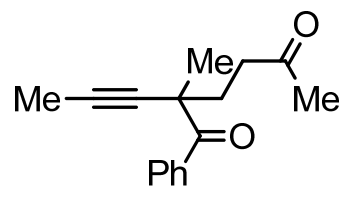

3-3g

Data for 3-3g: A colorless oil. IR (neat): $\bar{v}=2979,2936,1716,1682,1596,1446,1178$, 966, $716 \mathrm{~cm}^{-1} .{ }^{1} \mathrm{H} \mathrm{NMR}\left(\mathrm{CDCl}_{3}, 500 \mathrm{MHz}\right): \delta=1.52-1.54(\mathrm{~d}, J=9.0 \mathrm{~Hz}, 3 \mathrm{H}), 1.82-$ $1.83(\mathrm{~d}, J=9.0 \mathrm{~Hz}, 3 \mathrm{H}), 1.88-2.15(\mathrm{~m}, 1 \mathrm{H}), 2.15-2.17(\mathrm{~d}, J=8.5 \mathrm{~Hz}, 3 \mathrm{H}), 2.30-2.38$ (m, $1 \mathrm{H}), 2.51-2.59$ (m, $1 \mathrm{H}), 2.63-2.2 .71(\mathrm{~m}, 1 \mathrm{H}), 7.40-7.43$ (q, $J=8.0 \mathrm{~Hz}, 2 \mathrm{H}), 7.50-$ $7.53(\mathrm{t}, J=8.0 \mathrm{~Hz}, 1 \mathrm{H}), 8.24-8.27(\mathrm{t}, J=8.0 \mathrm{~Hz}, 2 \mathrm{H}) \mathrm{ppm} .{ }^{13} \mathrm{C} \mathrm{NMR}\left(\mathrm{CDCl}_{3}, 126\right.$ MHz): $\delta=3.7,26.7,29.9,33.7,39.8,45.3,81.2,82.5,127.9,129.7,132.5,135.4,199.5$, 208.2 ppm. $\mathrm{C}_{16} \mathrm{H}_{18} \mathrm{O}_{2}$ (242.13): calcd. C 79.31, H 7.49, found C 78.85, H 7.59.

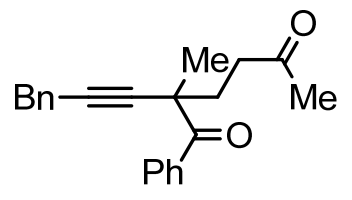

3-3h 
Data for 3-3h: A colorless oil. IR (neat): $\bar{v}=2982,2938,1714,1682,1597,1449,1243$, $1175,967,702 \mathrm{~cm}^{-1} .{ }^{1} \mathrm{H} \mathrm{NMR}\left(\mathrm{CDCl}_{3}, 500 \mathrm{MHz}\right): \delta=1.60(\mathrm{~s}, 3 \mathrm{H}), 1.96-2.02(\mathrm{~m}, 1 \mathrm{H})$, 2.14 (s, $3 \mathrm{H}), 2.37-2.43$ (m, $1 \mathrm{H}), 2.54-2.61$ (m, $1 \mathrm{H}), 2.67-2.74$ (m, $1 \mathrm{H}), 3.63$ (s, $2 \mathrm{H})$, 7.23-7.26 (m, 5 H), 7.37-7.40 (m, 2 H), 7.51-7.54 (m, $1 \mathrm{H}), 8.24-8.26(\mathrm{~m}, 2 \mathrm{H})$ ppm. ${ }^{13} \mathrm{C}$ NMR $\left(\mathrm{CDCl}_{3}, 126 \mathrm{MHz}\right): \delta=25.2,26.7,29.9,33.7,39.7,45.4,84.3,84.5$, 126.5, 127.8, 127.9, 128.4, 129.7, 132.5, 135.3, 136.4, 199.3, 208.1 ppm. HRMS (ESI): Calcd. for $\mathrm{C}_{22} \mathrm{H}_{23} \mathrm{O}_{2}\left[\mathrm{M}+\mathrm{H}^{+}\right]$calcd. 319.1693, found 319.1694.

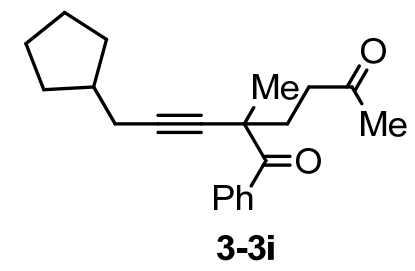

Data for 3-3i: A colorless oil. IR (neat): $\bar{v}=2951,2867,1717,1682,1447,1241,1176$, $965,715 \mathrm{~cm}^{-1} .{ }^{1} \mathrm{H}$ NMR ( $\left.\mathrm{CDCl}_{3}, 500 \mathrm{MHz}\right): \delta=1.16-1.22(\mathrm{~m}, 2 \mathrm{H}), 1.47-1.58(\mathrm{~m}, 7 \mathrm{H})$, 1.67-1.72 (m, 2 H), 1.74-2.01 (m, 2 H), 2.14 (s, 3 H), 2.17-2.19 (d, J=6.5 Hz, 2 H), 2.31-2.36 (m, $1 \mathrm{H}), 2.52-2.58(\mathrm{~m}, 1 \mathrm{H}), 2.64-2.71(\mathrm{~m}, 1 \mathrm{H}), 7.39-7.42(\mathrm{t}, J=7.5 \mathrm{~Hz}, 2$ H), 7.49-7.52 (t, $J=7.5 \mathrm{~Hz}, 1 \mathrm{H}), 8.25-8.27$ (d, $J=7.5 \mathrm{~Hz}, 2 \mathrm{H}) \mathrm{ppm} .{ }^{13} \mathrm{C} \mathrm{NMR}\left(\mathrm{CDCl}_{3}\right.$, $126 \mathrm{MHz}): \delta=24.6,25.2,26.8,29.9,31.9,33.7,38.9,39.7,45.3,82.1,86.6,127.8,129.7$, 132.4, 135.3, 199.5, $208.1 \mathrm{ppm}$.

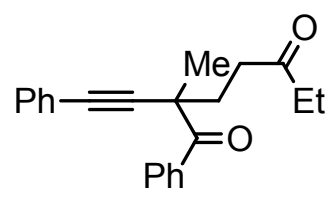

3-3j

Data for 3-3j: ${ }^{212}$ A colorless oil. IR (neat): $\bar{v}=2976,2937,1714,1682,1596,1445$, 1237, 1112, 972, 757, $691 \mathrm{~cm}^{-1} .{ }^{1} \mathrm{H}$ NMR $\left(\mathrm{CDCl}_{3}, 400 \mathrm{MHz}\right): \delta=1.01-1.04(\mathrm{t}, J=7.6$ Hz, $3 \mathrm{H}), 1.64$ (s, $3 \mathrm{H}), 2.02-2.09$ (m, $1 \mathrm{H}), 2.40-2.51$ (m, $3 \mathrm{H}), 2.54-2.62(\mathrm{~m}, 1 \mathrm{H})$, 
2.66-2.73 (m, $1 \mathrm{H}), 7.24-7.33$ (m, 5 H), 7.40-7.44 (t, $J=7.6 \mathrm{~Hz}, 2 \mathrm{H}), 7.50-7.54$ (t, $J=$ $7.2 \mathrm{~Hz}, 1 \mathrm{H}), 8.29-8.31$ (d, $J=7.2 \mathrm{~Hz}, 2 \mathrm{H}) \mathrm{ppm} .{ }^{13} \mathrm{C} \mathrm{NMR}\left(\mathrm{CDCl}_{3}, 101 \mathrm{MHz}\right): \delta=7.8$, 26.6, 33.7, 36.0, 38.3, 45.9, 86.7, 91.5, 122.8, 128.1, 128.3, 129.7, 131.3, 132.7, 135.3, 198.9, 210.6 ppm. HRMS (ESI): Calcd. for $\mathrm{C}_{22} \mathrm{H}_{22} \mathrm{O}_{2} \mathrm{Na}\left[\mathrm{M}+\mathrm{Na}^{+}\right]$calcd. 341.1512, found 341.1513.

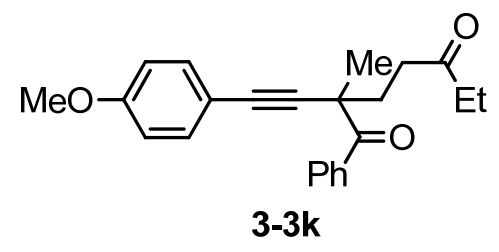

Data for 3-3k: ${ }^{212}$ A colorless oil. IR (neat): $\bar{v}=2975,2936,1714,1683,1606,1510$, 1457, 1249, 1109, 973, $716 \mathrm{~cm}^{-1} .{ }^{1} \mathrm{H}$ NMR $\left(\mathrm{CDCl}_{3}, 400 \mathrm{MHz}\right): \delta=0.93-0.97(\mathrm{t}, J=7.2$ Hz, $3 \mathrm{H}), 1.55$ (s, $3 \mathrm{H}), 1.92-1.99$ (m, $1 \mathrm{H}), 2.34-2.46$ (m, $3 \mathrm{H}), 2.46-2.54(\mathrm{~m}, 1 \mathrm{H})$, 2.58-2.67 (m, $1 \mathrm{H}), 3.694$ (s, $3 \mathrm{H}), 6.71-6.73(\mathrm{~d}, J=8.8 \mathrm{~Hz}, 2 \mathrm{H}), 7.17-7.20$ (d, $J=8.8$ Hz, 2 H), 7.33-7.37 (t, $J=7.2 \mathrm{~Hz}, 2 \mathrm{H}), 7.42-7.46$ (t, $J=7.2 \mathrm{~Hz}, 1 \mathrm{H}), 8.22-8.24$ (d, $J=$ $7.2 \mathrm{~Hz}, 2 \mathrm{H}) \mathrm{ppm} .{ }^{13} \mathrm{C} \mathrm{NMR}\left(\mathrm{CDCl}_{3}, 101 \mathrm{MHz}\right): \delta=7.8,26.6,33.7,36.0,38.4,45.9$, $55.2,86.5,90.0,113.9,114.9,128.0,129.7,132.6,132.7,135.4,159.6,199.1,210.7 \mathrm{ppm}$. HRMS (ESI): Calcd. for $\mathrm{C}_{23} \mathrm{H}_{24} \mathrm{O}_{3} \mathrm{Na}\left[\mathrm{M}+\mathrm{Na}^{+}\right]$371.1618, found 371.1624 .

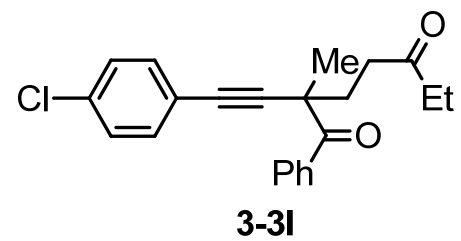

Data for 3-31: ${ }^{212}$ A colorless oil. IR (neat): $\bar{v}=2976,2836,1715,1684,1490,1237$, 1091, 972, $715 \mathrm{~cm}^{-1} .{ }^{1} \mathrm{H}$ NMR $\left(\mathrm{CDCl}_{3}, 500 \mathrm{MHz}\right): \delta=0.98-1.04(\mathrm{t}, J=7.6 \mathrm{~Hz}, 3 \mathrm{H})$, 1.62 (s, $3 \mathrm{H}), 2.00-2.07$ (m, $1 \mathrm{H}), 2.38-2.50(\mathrm{~m}, 3 \mathrm{H}), 2.53-2.57$ (m, $1 \mathrm{H}), 2.62-2.67$ (m, $1 \mathrm{H}), 7.22(\mathrm{~s}, 4 \mathrm{H}), 7.39-7.43(\mathrm{t}, J=7.6 \mathrm{~Hz}, 2 \mathrm{H}), 7.49-7.53(\mathrm{t}, J=7.2 \mathrm{~Hz}, 1 \mathrm{H}), 8.24-$ 
$8.25(\mathrm{~d}, J=7.6 \mathrm{~Hz}, 2 \mathrm{H}) \mathrm{ppm} .{ }^{13} \mathrm{C} \mathrm{NMR}\left(\mathrm{CDCl}_{3}, 126 \mathrm{MHz}\right): \delta=7.8,26.4,33.6,36.0$, $38.3,45.9,85.5,92.6,121.3,128.1,128.6,129.6,132.5,132.8,134.3,135.3,198.6,210.5$ ppm. HRMS (ESI): Calcd. for $\mathrm{C}_{22} \mathrm{H}_{21} \mathrm{ClO}_{2} \mathrm{Na}\left[\mathrm{M}+\mathrm{Na}^{+}\right]$375.1122, found 375.1126.

\section{Spectroscopic data of compounds 3-4}

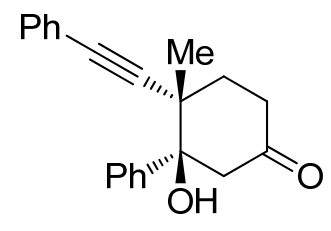

3-4a

Data for 3-4a: A colorless oil. IR (neat): $\bar{v}=3423,2960,2934,1716,1685,1491,1217$, 1060, 758, $715 \mathrm{~cm}^{-1} .{ }^{1} \mathrm{H}$ NMR (CDCl $\left.3,500 \mathrm{MHz}\right): \delta=1.19(\mathrm{~s}, 3 \mathrm{H}), 2.04-2.10(\mathrm{~m}, 1 \mathrm{H})$, 2.38-2.51 (m, $3 \mathrm{H}), 2.55-2.56(\mathrm{~d}, J=1.6 \mathrm{~Hz}, 1 \mathrm{H}), 2.94-3.02(\mathrm{~m}, 1 \mathrm{H}), 3.80-3.83$ (d, $J=$ 14.0 Hz, $1 \mathrm{H}), 7.31-7.34(\mathrm{~m}, 4 \mathrm{H}), 7.36-7.41(\mathrm{~m}, 4 \mathrm{H}), 7.63-7.64$ (d, J= 7.5 Hz, $2 \mathrm{H})$ ppm. ${ }^{13} \mathrm{C}$ NMR $\left(\mathrm{CDCl}_{3}, 126 \mathrm{MHz}\right): \delta=22.3,35.9,39.0,41.3,52.2,80.3,84.4,93.2$, 123.1, 126.5, 127.6, 128.2, 128.4, 131.4, 143.2, 210.6 ppm. HRMS (ESI): Calcd. for $\mathrm{C}_{21} \mathrm{H}_{20} \mathrm{O}_{2} \mathrm{Na}\left[\mathrm{M}+\mathrm{Na}^{+}\right]$327.1356, found 327.1360.

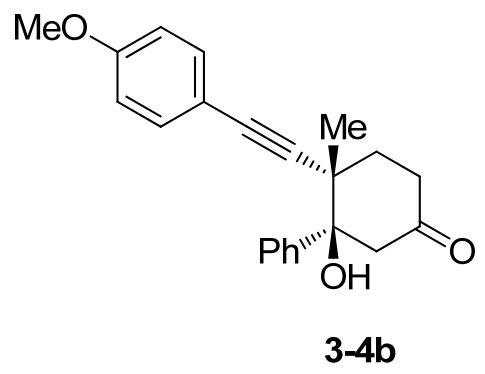

Data for 3-4b: A colorless oil. IR (neat): $\bar{v}=3460,2960,2936,1708,1606,1509,1248$, $1165,1032,732,701 \mathrm{~cm}^{-1} .{ }^{1} \mathrm{H}$ NMR $\left(\mathrm{CDCl}_{3}, 500 \mathrm{MHz}\right): \delta=1.18(\mathrm{~s}, 3 \mathrm{H}), 1.65-1.66(\mathrm{~m}$, $1 \mathrm{H}), 2.07-2.09$ (m, $1 \mathrm{H}), 2.39-2.50$ (m, $3 \mathrm{H}), 2.97-3.03$ (m, $1 \mathrm{H}), 3.83$ (s, $3 \mathrm{H}), 3.83-$ $3.85(\mathrm{~d}, J=11.0 \mathrm{~Hz}, 1 \mathrm{H}), 6.84-6.85(\mathrm{~d}, J=9.0 \mathrm{~Hz}, 2 \mathrm{H}), 7.31-7.39$ (m, $5 \mathrm{H}), 7.62-7.63$ 
(m, $2 \mathrm{H})$ ppm. ${ }^{13} \mathrm{C} \mathrm{NMR}\left(\mathrm{CDCl}_{3}, 126 \mathrm{MHz}\right): \delta=22.4,36.0,39.1,41.2,52.3,55.3,80.4$, 84.3, 91.6, 114.0, 115.2, 126.5, 127.6, 132.8, 143.2, 159.5, 210.4 ppm. HRMS (ESI): Calcd. for $\mathrm{C}_{22} \mathrm{H}_{23} \mathrm{O}_{3}\left[\mathrm{M}+\mathrm{H}^{+}\right]$335.1642, found 335.1646.

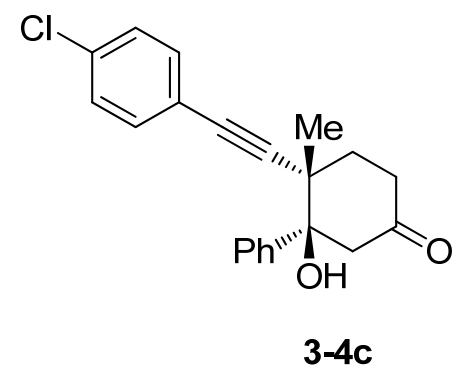

Data for 3-4c: A colorless oil. IR (neat): $\bar{v}=3428,2958,2934,1707,1489,1091,909$, 828, $701 \mathrm{~cm}^{-1} .{ }^{1} \mathrm{H}$ NMR $\left(\mathrm{CDCl}_{3}, 500 \mathrm{MHz}\right): \delta=1.20$ (s, $\left.3 \mathrm{H}\right), 2.06-2.09$ (m, $\left.1 \mathrm{H}\right), 2.41-$ $2.51(\mathrm{~m}, 3 \mathrm{H}), 2.60-2.66(\mathrm{~m}, 1 \mathrm{H}), 2.92-2.98(\mathrm{~m}, 1 \mathrm{H}), 3.77-3.81(\mathrm{~d}, J=14.5 \mathrm{~Hz}, 1 \mathrm{H})$, 7.28-7.41 (m, $7 \mathrm{H}), 7.61-7.63(\mathrm{~d}, J=8.0 \mathrm{~Hz}, 2 \mathrm{H}) \mathrm{ppm} .{ }^{13} \mathrm{C} \mathrm{NMR}\left(\mathrm{CDCl}_{3}, 126\right.$ MHz): $\delta=22.6,36.2,39.2,41.6,52.4,80.4,83.7,94.5,121.8,126.7,127.8,127.9,128.9$, 132.9, 134.5, 143.3, 210.7 ppm. HRMS (ESI): Calcd. for $\mathrm{C}_{21} \mathrm{H}_{19} \mathrm{ClO}_{2} \mathrm{Na}\left[\mathrm{M}+\mathrm{Na}^{+}\right]$ 361.0966, found 361.0968.

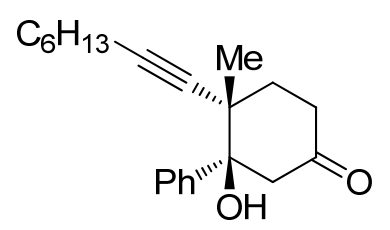

$3-4 d$

Data for 3-4d: A colorless oil. IR (neat): $\bar{v}=3433,2955,2929,2858,1715,1447,701$ $\mathrm{cm}^{-1} .{ }^{1} \mathrm{H}$ NMR $\left(\mathrm{CDCl}_{3}, 500 \mathrm{MHz}\right): \delta=0.89-0.90(\mathrm{~m}, 3 \mathrm{H}), 1.07(\mathrm{~s}, 3 \mathrm{H}), 1.28-1.39(\mathrm{~m}, 7$ H), 1.50-1.54 (m, 2 H), 1.90-1.96 (m, 1 H), 2.20-2.27 (t, J = 6.5 Hz, 2 H), 2.33-2.40 (m, $3 \mathrm{H}), 2.89-2.93(\mathrm{~m}, 1 \mathrm{H}), 3.74-3.76(\mathrm{~d}, J=14.0 \mathrm{~Hz}, 1 \mathrm{H}), 7.27-7.35$ (m, $3 \mathrm{H}), 7.57-7.58$ (d, $J=7.0 \mathrm{~Hz}, 2 \mathrm{H}) \mathrm{ppm} .{ }^{13} \mathrm{C} \mathrm{NMR}\left(\mathrm{CDCl}_{3}, 126 \mathrm{MHz}\right): \delta=14.3,18.9,22.8,22.9,28.9$, 
$29.1,31.5,36.5,39.2,40.9,52.5,80.5,83.8,84.8,126.8,127.6,127.7,143.6,211.2 \mathrm{ppm}$. HRMS (ESI): Calcd. for $\mathrm{C}_{21} \mathrm{H}_{28} \mathrm{O}_{2} \mathrm{Na}\left[\mathrm{M}+\mathrm{Na}^{+}\right]$335.1982, found 335.1985 .

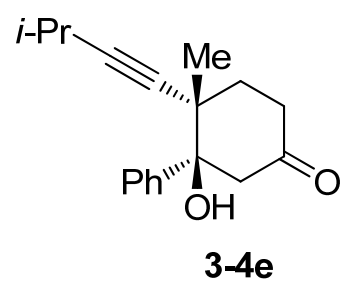

Data for 3-4e: A colorless oil. IR (neat): $\bar{v}=3419,2967,2933,1714,1656,1321,967$, $701 \mathrm{~cm}^{-1} .{ }^{1} \mathrm{H} \mathrm{NMR}\left(\mathrm{CDCl}_{3}, 400 \mathrm{MHz}\right): \delta=1.03(\mathrm{~s}, 3 \mathrm{H}), 1.15-1.17(\mathrm{t}, J=6.4 \mathrm{~Hz}, 6 \mathrm{H})$, 1.87-1.93 (m, 1 H), 2.07-2.11 (m, $1 \mathrm{H}), 2.29-2.39$ (m, 3 H), 2.51-2.59 (m, $1 \mathrm{H}), 2.51-$ 2.85-2.93 (m, $1 \mathrm{H}), 3.72-3.75(\mathrm{~d}, J=14.0 \mathrm{~Hz}, 1 \mathrm{H}), 7.24-7.34(\mathrm{~m}, 3 \mathrm{H}), 7.54-7.56$ (d, $J=7.2 \mathrm{~Hz}, 2 \mathrm{H}) \mathrm{ppm} .{ }^{13} \mathrm{C} \mathrm{NMR}\left(\mathrm{CDCl}_{3}, 101 \mathrm{MHz}\right): \delta=20.5,22.5,23.1,23.2,36.1$, 38.9, 40.4, 52.2, 80.3, 82.9, 89.9, 126.5, 127.3, 127.4, 143.3, 210.8 ppm. HRMS (ESI): Calcd. for $\mathrm{C}_{18} \mathrm{H}_{22} \mathrm{O}_{2} \mathrm{Na}\left[\mathrm{M}+\mathrm{Na}^{+}\right]$293.1512, found 293.1516.

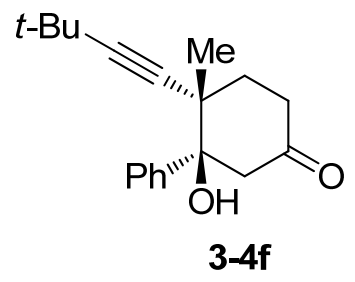

Data for 3-4f: A colorless oil. IR (neat): $\bar{v}=3386,2963,2929,2866,1703,1636,1360$, 915, $701 \mathrm{~cm}^{-1} .{ }^{1} \mathrm{H}$ NMR $\left(\mathrm{CDCl}_{3}, 500 \mathrm{MHz}\right): \delta=1.04$ (s, $\left.3 \mathrm{H}\right), 1.24(\mathrm{~s}, 9 \mathrm{H}), 1.88-1.92$ (m, $1 \mathrm{H}), 2.32-2.39$ (m, $4 \mathrm{H}), 2.86-2.92(\mathrm{~m}, 1 \mathrm{H}), 3.73-3.76$ (d, J = $15.0 \mathrm{~Hz}, 1 \mathrm{H}), 7.29-$ $7.36(\mathrm{~m}, 3 \mathrm{H}), 7.58-7.60(\mathrm{~d}, J=8.0 \mathrm{~Hz}, 2 \mathrm{H}) \mathrm{ppm} .{ }^{13} \mathrm{C} \mathrm{NMR}\left(\mathrm{CDCl}_{3}, 101 \mathrm{MHz}\right): \delta=$ $22.5,27.4,30.4,36.2,38.9,40.3,52.2,80.3,82.2,92.7,126.6,126.9,127.3,127.4,143.3$, 210.9 ppm. HRMS (ESI): Calcd. for $\mathrm{C}_{19} \mathrm{H}_{24} \mathrm{O}_{2} \mathrm{Na}\left[\mathrm{M}+\mathrm{Na}^{+}\right]$307.1669, found 307.1671. 


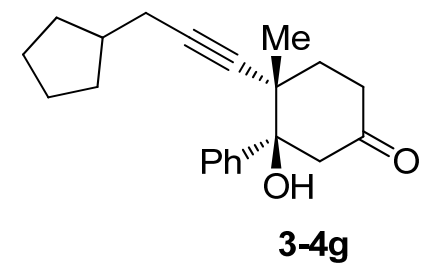

Data for 3-4g: A colorless oil. IR (neat): $\bar{v}=3446,2951,2867,1707,1447,1373,1053$, 970, $701 \mathrm{~cm}^{-1} .{ }^{1} \mathrm{H}$ NMR $\left(\mathrm{CDCl}_{3}, 400 \mathrm{MHz}\right): \delta=1.06(\mathrm{~s}, 3 \mathrm{H}), 1.54-1.62(\mathrm{~m}, 5 \mathrm{H}), 1.76-$ $1.79(\mathrm{~m}, 2 \mathrm{H}), 1.90-1.96(\mathrm{~m}, 1 \mathrm{H}), 2.01-2.08(\mathrm{~m}, 1 \mathrm{H}), 2.14(\mathrm{~s}, 1 \mathrm{H}), 2.20-2.22(\mathrm{~d}, J=$ $6.0 \mathrm{~Hz}, 2 \mathrm{H}), 2.32-2.42$ (m, $3 \mathrm{H}), 2.87-2.96(\mathrm{~m}, 1 \mathrm{H}), 3.73-3.77$ (d, J=14.4 Hz, $1 \mathrm{H})$, 7.28-7.37 (m, $3 \mathrm{H}), 7.56-7.58(\mathrm{~d}, J=8.0 \mathrm{~Hz}, 2 \mathrm{H}) \mathrm{ppm} .{ }^{13} \mathrm{C} \mathrm{NMR}\left(\mathrm{CDCl}_{3}, 101\right.$ MHz): $\delta=22.6,24.7,31.5,32.1,36.3,39.0,39.2,40.6,47.6,52.4,80.3,83.6,84.0,126.4$, 127.0, 127.4, 127.5, 143.3, 210.7 ppm. HRMS (ESI): Calcd. for $\mathrm{C}_{21} \mathrm{H}_{26} \mathrm{O}_{2} \mathrm{Na}\left[\mathrm{M}+\mathrm{Na}^{+}\right]$ 333.1825, found 333.1827 .

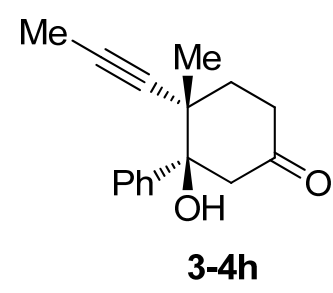

Data for 3-4h: A colorless oil. IR (neat): $\bar{v}=3372,2974,2936,1715,1558,1338,1069$, 970, 766, $703 \mathrm{~cm}^{-1} .{ }^{1} \mathrm{H} \mathrm{NMR}\left(\mathrm{CDCl}_{3}, 400 \mathrm{MHz}\right): \delta=0.97$ (s, $\left.3 \mathrm{H}\right), 1.76(\mathrm{~s}, 3 \mathrm{H}), 1.81-$ $1.85(\mathrm{~m}, 1 \mathrm{H}), 2.23-2.31(\mathrm{~m}, 3 \mathrm{H}), 2.41(\mathrm{~s}, 1 \mathrm{H}), 2.46-2.85(\mathrm{~m}, 1 \mathrm{H}), 3.63-3.66(\mathrm{~d}, J=$ 14.4 Hz, $1 \mathrm{H}), 7.18-7.29(\mathrm{~m}, 3 \mathrm{H}), 7.46-7.49(\mathrm{~d}, J=8.0 \mathrm{~Hz}, 2 \mathrm{H}) \mathrm{ppm} .{ }^{13} \mathrm{C} \mathrm{NMR}\left(\mathrm{CDCl}_{3}\right.$, $101 \mathrm{MHz}): \delta=3.5,22.5,36.1,39.0,40.6,52.2,79.7,80.2,82.7,126.5,127.4,127.5$, 143.4, 211.1 ppm. HRMS (ESI): Calcd. for $\mathrm{C}_{16} \mathrm{H}_{18} \mathrm{O}_{2} \mathrm{Na}\left[\mathrm{M}+\mathrm{Na}^{+}\right]$265.1199, found 265.1202. 


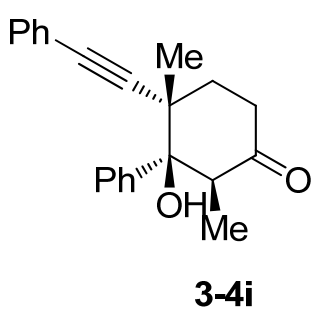

Data for 3-4i: A colorless oil. IR (neat): $\bar{v}=3501,2976,2937,1711,1489,1262,1099$, 994, 757, $692 \mathrm{~cm}^{-1} .{ }^{1} \mathrm{H}$ NMR ( $\left.\mathrm{CDCl}_{3}, 400 \mathrm{MHz}\right): \delta=0.73-0.75(\mathrm{~d}, J=6.8 \mathrm{~Hz}, 3 \mathrm{H}), 1.05$ (s, 3 H), 1.97-2.03 (m, 2 H), 2.33-2.46 (m, 2 H), 2.95-3.03 (td, J=14, $6.0 \mathrm{~Hz}, 1 \mathrm{H})$, 3.67-3.73 (q, $J=6.8 \mathrm{~Hz}, 1 \mathrm{H}), 7.17-7.35(\mathrm{~m}, 7 \mathrm{H}), 7.45-7.55(\mathrm{~m}, 2 \mathrm{H}) \mathrm{ppm} .{ }^{13} \mathrm{C}$ NMR $\left(\mathrm{CDCl}_{3}, 101 \mathrm{MHz}\right): \delta=8.3,23.0,36.1,39.1,42.3,50.2,83.6,84.7,93.6,123.2,127.3$, 128.1, 128.3, 131.4, 141.6, 211.2 ppm. HRMS (ESI): Calcd. for $\mathrm{C}_{22} \mathrm{H}_{23} \mathrm{O}_{2}\left[\mathrm{M}+\mathrm{H}^{+}\right]$ 319.1693, found 319.1695.

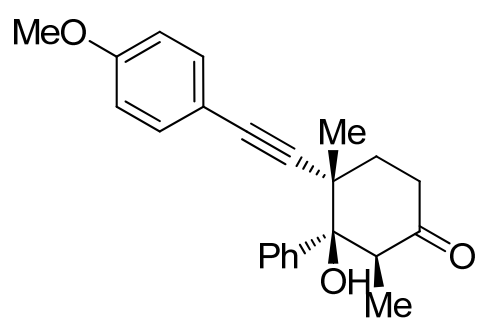

3-4j

Data for 3-4j: A colorless oil. IR (neat): $\bar{v}=3502,2972,2936,1715,1653,1606,1457$, 1247, 1031, 832, 737, $702 \mathrm{~cm}^{-1} .{ }^{1} \mathrm{H}$ NMR ( $\left.\mathrm{CDCl}_{3}, 400 \mathrm{MHz}\right): \delta=0.72-0.74(\mathrm{~d}, J=6.8$ Hz, 3 H), 1.03 (s, 3 H), 1.96-2.00 (m, 1 H), 2.05 (s, 1 H), 2.31-2.46 (m, 2 H), 2.95-3.03 (td, $J=13.6,6.4 \mathrm{~Hz}, 1 \mathrm{H}), 3.67-3.71(\mathrm{q}, J=6.8 \mathrm{~Hz}, 1 \mathrm{H}), 3.74(\mathrm{~s}, 3 \mathrm{H}), 6.77-6.79(\mathrm{~d}, J=$ $8.4 \mathrm{~Hz}, 2 \mathrm{H}), 7.18-7.30(\mathrm{~m}, 5 \mathrm{H}), 7.49-7.55(\mathrm{~m}, 2 \mathrm{H}) \mathrm{ppm} .{ }^{13} \mathrm{C} \mathrm{NMR}\left(\mathrm{CDCl}_{3}, 101\right.$ $\mathrm{Hz}): \delta=8.3,23.1,36.2,39.2,42.3,50.1,55.3,83.7,84.5,92.0,114.0,115.3,127.2$, 132.8, 141.7, 159.5, 211.3 ppm. HRMS (ESI): Calcd. for $\mathrm{C}_{23} \mathrm{H}_{25} \mathrm{O}_{3}\left[\mathrm{M}+\mathrm{H}^{+}\right]$349.1798, found 349.1801 . 


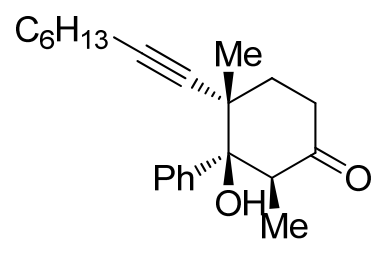

3-4k

Data for 3-4k: A colorless oil. IR (neat): $\bar{v}=3488,2932,2858,1711,1652,1456,1340$, 1159, 936, $701 \mathrm{~cm}^{-1} .{ }^{1} \mathrm{H} \mathrm{NMR}\left(\mathrm{CDCl}_{3}, 400 \mathrm{MHz}\right): \delta=0.70-0.72(\mathrm{~d}, J=7.2 \mathrm{~Hz}, 3 \mathrm{H})$, 0.80-0.83 (t, J=7.2 Hz, $3 \mathrm{H}), 0.92(\mathrm{~s}, 3 \mathrm{H}), 1.15-1.35(\mathrm{~m}, 7 \mathrm{H}), 1.43-1.50(\mathrm{~m}, 2 \mathrm{H})$, $1.82-1.87$ (m, $2 \mathrm{H}), 2.14-2.17$ (t, $J=7.2 \mathrm{~Hz}, 2 \mathrm{H}), 2.23-2.31$ (td, $J=13.2,4.0 \mathrm{~Hz}, 1 \mathrm{H})$, 2.35-2.40 (m, $1 \mathrm{H}), 2.87-2.96(\mathrm{td}, J=13.6,6.4 \mathrm{~Hz}, 1 \mathrm{H}), 3.61-3.66(\mathrm{q}, J=6.8 \mathrm{~Hz}, 1 \mathrm{H})$, 7.19-7.28 (m, $3 \mathrm{H}), 7.46$ (br. s, $2 \mathrm{H}) \mathrm{ppm} .{ }^{13} \mathrm{C} \mathrm{NMR}\left(\mathrm{CDCl}_{3}, 101 \mathrm{MHz}\right): \delta=8.3,14.0$, $18.8,22.5,23.3,28.6,28.8,31.3,36.4,39.1,41.7,50.0,83.6,83.9,84.8,125.9,127.1$, 127.9, 141.7, 211.6 ppm. HRMS (ESI): Calcd. for $\mathrm{C}_{22} \mathrm{H}_{31} \mathrm{O}_{2}\left[\mathrm{M}+\mathrm{H}^{+}\right]$327.2319, found 327.2323 .

\section{Spectroscopic data of compounds 3-5.}

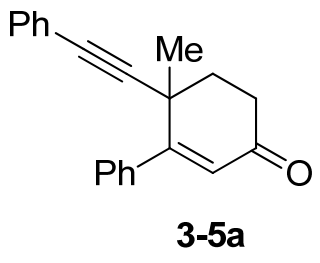

Data for 3-5a: A colorless oil. IR (neat): $\bar{v}=3058,2931,1714,1681,1490,1330,1265$, 1056, $756 \mathrm{~cm}^{-1} .{ }^{1} \mathrm{H}$ NMR $\left(\mathrm{CDCl}_{3}, 500 \mathrm{MHz}\right): \delta=1.44(\mathrm{~s}, 3 \mathrm{H}), 2.19-2.25(\mathrm{~m}, 1 \mathrm{H})$, 2.38-2.42 (m, $1 \mathrm{H}), 2.56-2.61$ (m, $1 \mathrm{H}), 2.99-3.06$ (m, $1 \mathrm{H}), 6.06$ (s, $1 \mathrm{H}), 7.32-7.33$ (m, $3 \mathrm{H}), 7.38-7.39$ (m, $3 \mathrm{H}), 7.43-7.45$ (m, $2 \mathrm{H}), 7.62-7.63$ (m, $2 \mathrm{H}) \mathrm{ppm} .{ }^{13} \mathrm{C} \mathrm{NMR}\left(\mathrm{CDCl}_{3}\right.$, $126 \mathrm{MHz}): \delta=28.1,35.2,35.3,38.9,83.1,91.6,122.9,127.4,127.6,128.2,128.3,128.8$, 
131.6, 138.7, 163.6, 198.8 ppm. HRMS (ESI): Calcd. for $\mathrm{C}_{21} \mathrm{H}_{19} \mathrm{O}\left[\mathrm{M}+\mathrm{H}^{+}\right]$287.1430, found 287.1434 .

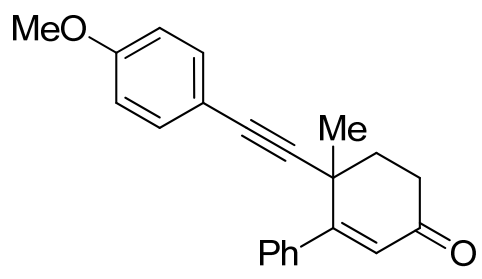

$3-5 b$

Data for 3-5b: A colorless oil. IR (neat): $\bar{v}=2930,2837,1673,1606,1456,1248,1172$, 1032, $731 \mathrm{~cm}^{-1} .{ }^{1} \mathrm{H}$ NMR $\left(\mathrm{CDCl}_{3}, 500 \mathrm{MHz}\right): \delta=1.43(\mathrm{~s}, 3 \mathrm{H}), 2.19-2.26(\mathrm{~m}, 1 \mathrm{H})$, 2.38-2.42 (m, $1 \mathrm{H}), 2.56-2.60(\mathrm{~m}, 1 \mathrm{H}), 3.01-3.09(\mathrm{~m}, 1 \mathrm{H}), 3.82(\mathrm{~s}, 3 \mathrm{H}), 6.06(\mathrm{~s}, 1 \mathrm{H})$, 6.86-6.87 (d, J=9.0 Hz, $2 \mathrm{H}), 7.38-7.40(\mathrm{~m}, 5 \mathrm{H}), 7.63-7.64(\mathrm{~d}, J=3.5 \mathrm{~Hz}, 2 \mathrm{H})$ ppm. 13C NMR $\left(\mathrm{CDCl}_{3}, 126 \mathrm{MHz}\right): \delta=28.4,35.5,35.6,39.3,55.6,83.2,90.3,114.2$, $115.3,127.7,127.8,128.5,129.1,133.3,139.1,159.9,164.4,199.2$ ppm. HRMS (ESI): Calcd. for $\mathrm{C}_{22} \mathrm{H}_{21} \mathrm{O}_{2}\left[\mathrm{M}+\mathrm{H}^{+}\right]$317.1536, found 317.1538.

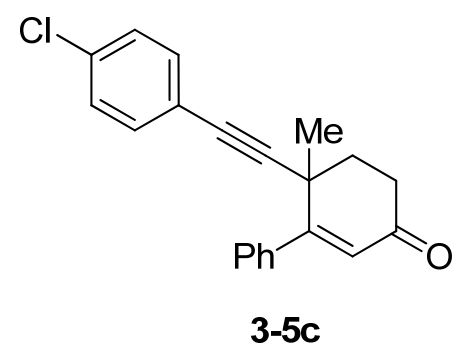

Data for 3-5c: A colorless oil. IR (neat): $\bar{v}=2955,2928,1676,1597,1489,1264,1091$, 828, 766, $701 \mathrm{~cm}^{-1} .{ }^{1} \mathrm{H}$ NMR $\left(\mathrm{CDCl}_{3}, 500 \mathrm{MHz}\right): \delta=1.45(\mathrm{~s}, 3 \mathrm{H}), 2.21-2.27(\mathrm{~m}, 1 \mathrm{H})$, 2.40-2.43 (m, $1 \mathrm{H}), 2.58-2.61(\mathrm{~m}, 1 \mathrm{H}), 2.97-3.04(\mathrm{~m}, 1 \mathrm{H}), 6.07$ (s, $1 \mathrm{H})$, 7.30-7.41 (m, $7 \mathrm{H}), 7.60-7.61(\mathrm{~d}, J=3.5 \mathrm{~Hz}, 2 \mathrm{H}) \mathrm{ppm} .{ }^{13} \mathrm{C} \mathrm{NMR}\left(\mathrm{CDCl}_{3}, 126 \mathrm{MHz}\right): \delta=28.3,35.4$, $35.6,39.2,82.3,92.9,121.6,127.6,128.0,128.5,128.9,129.2,133.1,134.6,138.9,163.6$, 198.9 ppm. HRMS (ESI): Calcd. for $\mathrm{C}_{21} \mathrm{H}_{18} \mathrm{ClO}\left[\mathrm{M}+\mathrm{H}^{+}\right]$321.1041, found 321.1045. 


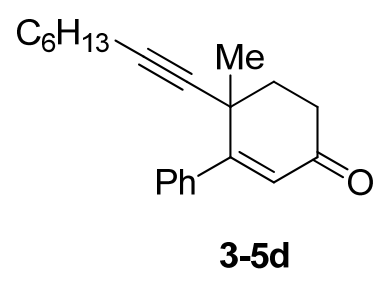

Data for 3-5d: A colorless oil. IR (neat): $\bar{v}=2930,2858,1683,1456,1264,701 \mathrm{~cm}^{-}$ 1. ${ }^{1} \mathrm{H}$ NMR $\left(\mathrm{CDCl}_{3}, 500 \mathrm{MHz}\right): \delta=0.87-0.89(\mathrm{t}, J=7.0 \mathrm{~Hz}, 3 \mathrm{H}), 1.25-1.32(\mathrm{~m}, 7 \mathrm{H})$, 1.35-1.42 (m, 2 H), 1.49-1.55 (m, 2 H), 2.06-2.13 (m, 1 H), 2.21-2.24 (m, 3 H), 2.47$2.52(\mathrm{~m}, 1 \mathrm{H}), 2.91-2.98(\mathrm{~m}, 1 \mathrm{H}), 5.97$ (s, $1 \mathrm{H}), 7.35-7.37$ (m, $3 \mathrm{H}), 7.56-7.58(\mathrm{~m}, 2 \mathrm{H})$ ppm. $\left.{ }^{13} \mathrm{C} \mathrm{NMR} \mathrm{(} \mathrm{CDCl}_{3}, 126 \mathrm{MHz}\right): \delta=14.0,18.7,22.5,28.4,28.5,28.7,31.2,34.7,35.2$, 39.3, 82.3, 83.4, 127.2, 127.4, 128.1, 128.7, 138.9, 164.4, 199.2 ppm. HRMS (ESI): Calcd. for $\mathrm{C}_{21} \mathrm{H}_{27} \mathrm{O}\left[\mathrm{M}+\mathrm{H}^{+}\right]$295.2056, found 295.2059.

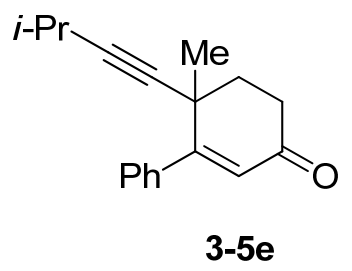

Data for 3-5e: A colorless oil. IR (neat): $\bar{v}=2968,2930,2870,1675,1653,1558,1320$, 1150, $701 \mathrm{~cm}^{-1} .{ }^{1} \mathrm{H}$ NMR $\left(\mathrm{CDCl}_{3}, 400 \mathrm{MHz}\right): \delta=1.10(\mathrm{~s}, 6 \mathrm{H}), 1.22(\mathrm{~s}, 3 \mathrm{H}), 1.98-2.05$ (m, $1 \mathrm{H}), 2.14-2.16(\mathrm{~m}, 1 \mathrm{H}), 2.40-2.45$ (m, $1 \mathrm{H}), 2.51-2.54(\mathrm{~m}, 1 \mathrm{H}), 2.83-2.90$ (m, 1 H), 5.89 (s, $1 \mathrm{H}), 7.29$ (s, $3 \mathrm{H}), 7.50$ (s, $2 \mathrm{H}) \mathrm{ppm} .{ }^{13} \mathrm{C} \mathrm{NMR}\left(\mathrm{CDCl}_{3}, 101 \mathrm{MHz}\right): \delta=20.6$, $23.0,28.3,34.6,35.2,39.2,81.5,88.8,127.2,127.4,128.1,128.7,138.9,164.6,199.3$ ppm. HRMS (ESI): Calcd. for $\mathrm{C}_{18} \mathrm{H}_{21} \mathrm{O}\left[\mathrm{M}+\mathrm{H}^{+}\right]$253.1587, found 253.1589 .

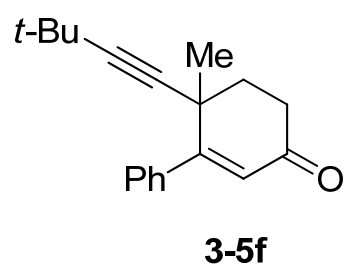


Data for 3-5f: A colorless oil. IR (neat): $\bar{v}=2967,2929,2867,1677,1599,1270,1150$, $701 \mathrm{~cm}^{-1} .{ }^{1} \mathrm{H} \mathrm{NMR}\left(\mathrm{CDCl}_{3}, 500 \mathrm{MHz}\right): \delta=1.24(\mathrm{~s}, 9 \mathrm{H}), 1.29(\mathrm{~s}, 3 \mathrm{H}), 2.06-2.13(\mathrm{td}, J=$ 13.0, 4.0 Hz, $1 \mathrm{H}), 2.20-2.24(\mathrm{dt}, J=13.0,4.5 \mathrm{~Hz}, 1 \mathrm{H}), 2.48-2.53(\mathrm{dt}, J=16.5,4.0 \mathrm{~Hz}$, $1 \mathrm{H}), 2.89-2.96(\mathrm{~m}, 1 \mathrm{H}), 5.97(\mathrm{~s}, 1 \mathrm{H}), 7.36-7.38(\mathrm{t}, J=3.5 \mathrm{~Hz}, 3 \mathrm{H}), 7.57-7.59(\mathrm{~m}, 2 \mathrm{H})$ ppm. ${ }^{13} \mathrm{C}$ NMR $\left(\mathrm{CDCl}_{3}, 126 \mathrm{MHz}\right): \delta=27.2,28.1,30.7,34.3,34.9,38.9,80.5,91.4$, 126.8, 127.2, 127.7, 128.4, 138.7, 164.4, 198.9 ppm. HRMS (ESI): Calcd. for $\mathrm{C}_{19} \mathrm{H}_{23} \mathrm{O}$ $\left[\mathrm{M}+\mathrm{H}^{+}\right]$289.1563, found 289.1566.

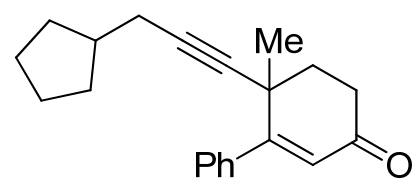

3-5g

Data for 3-5g: A colorless oil. IR (neat): $\bar{v}=2949,2865,1677,1558,1264,1151,766$, $701 \mathrm{~cm}^{-1} .{ }^{1} \mathrm{H}$ NMR $\left(\mathrm{CDCl}_{3}, 500 \mathrm{MHz}\right): \delta=1.25-1.29$ (m, $\left.2 \mathrm{H}\right), 1.31$ (s, $\left.3 \mathrm{H}\right), 1.54-1.66$ (m, 4 H), 1.76-1.82 (m, 2 H), 2.04-2.15 (m, 2 H), 2.23-2.27 (m, 3 H), 2.49-2.54 (m, 1 H), 2.94-3.05 (m, $1 \mathrm{H}), 5.99$ (s, $1 \mathrm{H}), 7.37-7.38$ (t, J = 3.5 Hz, $3 \mathrm{H}), 7.59-7.61$ (m, $2 \mathrm{H})$ ppm. $\left.{ }^{13} \mathrm{C} \mathrm{NMR} \mathrm{(} \mathrm{CDCl}_{3}, 126 \mathrm{MHz}\right): \delta=24.6,25.3,28.4,32.0,34.7,35.2,39.1,39.4,82.3$, 82.8, 127.2, 127.4, 128.1, 128.7, 138.9, 164.4, 199.2 ppm. HRMS (ESI): Calcd. for $\mathrm{C}_{21} \mathrm{H}_{25} \mathrm{O}\left[\mathrm{M}+\mathrm{H}^{+}\right]$293.1900, found 293.1906.

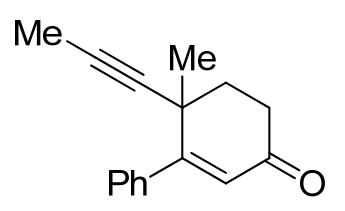

3-5h

Data for 3-5h: A colorless oil. IR (neat): $\bar{v}=2951,2922,1674,1616,1558,1456,1264$, 1151, 766, $701 \mathrm{~cm}^{-1} .{ }^{1} \mathrm{H}$ NMR $\left(\mathrm{CDCl}_{3}, 500 \mathrm{MHz}\right): \delta=1.29$ (s, $\left.3 \mathrm{H}\right), 1.89$ (s, $\left.3 \mathrm{H}\right), 2.07-$ $2.13(\mathrm{~m}, 1 \mathrm{H}), 2.22-2.26(\mathrm{~m}, 1 \mathrm{H}), 2.48-2.53(\mathrm{~m}, 1 \mathrm{H}), 2.93-2.99(\mathrm{~m}, 1 \mathrm{H}), 5.98(\mathrm{~s}, 1 \mathrm{H})$, 
7.37-7.39 (m, $3 \mathrm{H}), 7.57-7.58$ (m, $2 \mathrm{H}) \mathrm{ppm} .{ }^{13} \mathrm{C} \mathrm{NMR}\left(\mathrm{CDCl}_{3}, 126 \mathrm{MHz}\right): \delta=3.6,28.3$, $34.6,35.2,39.2,78.6,81.3,127.2,127.4,128.1,128.7,138.9,164.2,199.1$ ppm. HRMS (ESI): Calcd. for $\mathrm{C}_{16} \mathrm{H}_{17} \mathrm{O}\left[\mathrm{M}+\mathrm{H}^{+}\right]$225.1274, found 225.1278.

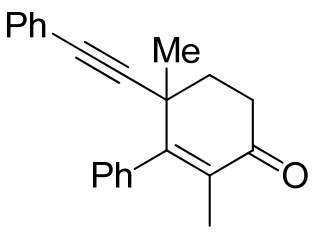

$3-5 i$

Data for 3-5i: A colorless oil. IR (neat): $\bar{v}=2928,2864,1675,1597,1443,1306,1013$, 892, 756, $704 \mathrm{~cm}^{-1} .{ }^{1} \mathrm{H} \mathrm{NMR}\left(\mathrm{CDCl}_{3}, 400 \mathrm{MHz}\right): \delta=1.18(\mathrm{~s}, 3 \mathrm{H}), 1.48(\mathrm{~s}, 3 \mathrm{H}), 2.07-$ $2.14(\mathrm{td}, J=13.2,4.0 \mathrm{~Hz}, 1 \mathrm{H}), 2.26-2.31(\mathrm{dt}, J=12.8,4.8 \mathrm{~Hz}, 1 \mathrm{H}), 2.52-2.58(\mathrm{dt}, J=$ 13.2, 4.4 Hz, $1 \mathrm{H}), 2.89-2.98(\mathrm{~m}, 1 \mathrm{H}), 7.16-7.32(\mathrm{~m}, 10 \mathrm{H}) \mathrm{ppm} .{ }^{13} \mathrm{C} \mathrm{NMR}\left(\mathrm{CDCl}_{3}, 101\right.$ MHz): $\delta=13.4,28.3,35.3,36.8,37.8,82.7,92.0,123.2,127.5,127.7,128.0,128.3$, 131.6, 132.1, 138.5, 158.2, 198.9 ppm. HRMS (ESI): Calcd. for $\mathrm{C}_{22} \mathrm{H}_{21} \mathrm{O}\left[\mathrm{M}+\mathrm{H}^{+}\right]$ 301.1587, found 301.1590.

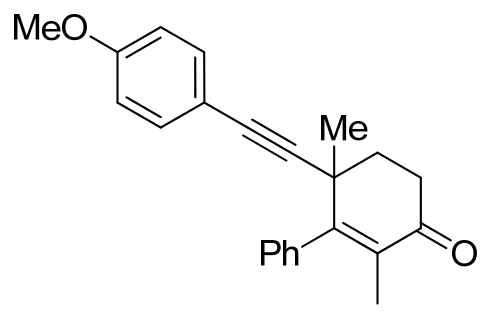

3-5j

Data for 3-5j: A colorless oil. IR (neat): $\bar{v}=2961,2929,1674,1606,1456,1291,1172$, 1032, 892, $731 \mathrm{~cm}^{-1}$. 1H NMR ( $\left.\mathrm{CDCl}_{3}, 400 \mathrm{MHz}\right): \delta=1.17$ (s, $\left.3 \mathrm{H}\right), 1.48(\mathrm{~s}, 3 \mathrm{H}), 2.06-$ $2.14(\mathrm{td}, J=13.2,4.0 \mathrm{~Hz}, 1 \mathrm{H}), 2.25-2.31(\mathrm{dt}, J=13.2,4.8 \mathrm{~Hz}, 1 \mathrm{H}), 2.51-2.57(\mathrm{dt}, J=$ 16.8, 4.0 Hz, $1 \mathrm{H}), 2.90-2.99$ (m, $1 \mathrm{H}), 3.72(\mathrm{~s}, 3 \mathrm{H}), 6.76-6.74(\mathrm{~d}, J=8.4 \mathrm{~Hz}, 2 \mathrm{H})$, 7.14-7.17 (m, 2 H), 7.24-7.27 (m, 3 H), 7.29-7.33 (t, $J=7.6 \mathrm{~Hz}, 2 \mathrm{H})$ ppm. ${ }^{13} \mathrm{C}$ NMR 
$\left(\mathrm{CDCl}_{3}, 101 \mathrm{MHz}\right): \delta=13.4,28.4,35.4,36.8,37.8,55.3,82.5,90.4,113.9,115.3,127.5$, 127.7, 128.0, 131.9, 132.9, 138.6, 158.4, 159.4, 199.1 ppm. HRMS (ESI): Calcd. for $\mathrm{C}_{23} \mathrm{H}_{22} \mathrm{O} 2 \mathrm{Na}\left[\mathrm{M}+\mathrm{Na}^{+}\right]$353.1512, found 353.1515.

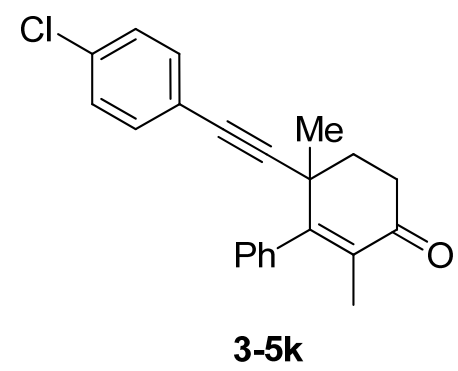

Data for 3-5k: A colorless oil. IR (neat): $\bar{v}=2966,2928,1674,1606,1456,1306,1089$, 1013, 828, $762 \mathrm{~cm}^{-1} .{ }^{1} \mathrm{H}$ NMR $\left(\mathrm{CDCl}_{3}, 400 \mathrm{MHz}\right): \delta=1.20$ (s, $\left.3 \mathrm{H}\right), 1.48(\mathrm{~s}, 3 \mathrm{H}), 2.08-$ $2.16(\mathrm{td}, J=12.8,4.0 \mathrm{~Hz}, 1 \mathrm{H}), 2.27-2.33(\mathrm{dt}, J=13.2,4.8 \mathrm{~Hz}, 1 \mathrm{H}), 2.53-2.59(\mathrm{td}, J=$ 17.2, 4.4 Hz, $1 \mathrm{H}), 2.87-2.96$ (m, $1 \mathrm{H}), 7.12-7.17$ (m, $2 \mathrm{H}), 7.18-7.23$ (m, $4 \mathrm{H}), 7.24-$ 7.27 (m, $1 \mathrm{H}), 7.30-7.34$ (m, $2 \mathrm{H}) \mathrm{ppm} .{ }^{13} \mathrm{C} \mathrm{NMR}\left(\mathrm{CDCl}_{3}, 101 \mathrm{MHz}\right): \delta=13.4,28.2$, $35.2,36.9,37.7,81.7,93.1,121.6,127.6,128.1,128.6,132.2,132.8,134.1,138.4,157.9$, 198.8 ppm. HRMS (ESI): Calcd. for $\mathrm{C}_{22} \mathrm{H}_{20} \mathrm{ClO}\left[\mathrm{M}+\mathrm{H}^{+}\right]$335.1197, found 335.1200.

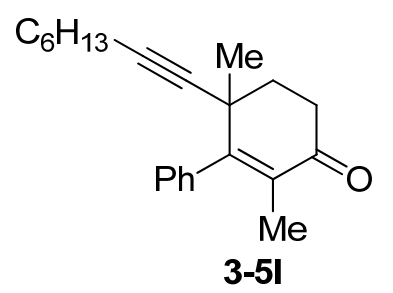

Data for 3-51: A colorless oil. IR (neat): $\bar{v}=2929,2858,1676,1617,1456,1306,1098$, 896, $766 \mathrm{~cm}^{-1}$. 1H NMR ( $\left.\mathrm{CDCl}_{3}, 400 \mathrm{MHz}\right): \delta=0.79-0.83(\mathrm{t}, J=6.8 \mathrm{~Hz}, 3 \mathrm{H}), 1.06$ (s, 3 H), 1.20-1.32 (m, 6 H), 1.38-1.45 (m, 5 H), 1.97-2.05 (td, $J=12.8,4.0 \mathrm{~Hz}, 1 \mathrm{H}), 2.10-$ $2.16(\mathrm{~m}, 3 \mathrm{H}), 2.46-2.52$ (dt, $J=16.8,4.0 \mathrm{~Hz}, 1 \mathrm{H}), 2.84-2.93$ (m, $1 \mathrm{H}), 7.13$ (br. s, $2 \mathrm{H}$ ), 7.22-7.32 (m, $3 \mathrm{H})$ ppm. ${ }^{13} \mathrm{C}$ NMR $\left(\mathrm{CDCl}_{3}, 101 \mathrm{MHz}\right): \delta=13.3,14.0,18.7,22.5,28.5$, 
28.6, 28.8, 31.3, 35.4, 36.2, 38.2, 82.4, 82.8, 127.3, 127.6, 127.8, 131.4, 138.7, 159.1, 199.4 ppm. HRMS (ESI): Calcd. for $\mathrm{C}_{22} \mathrm{H}_{29} \mathrm{O}\left[\mathrm{M}+\mathrm{H}^{+}\right]$309.2213, found 309.2216.

\section{${ }^{1} \mathrm{H},{ }^{13} \mathrm{C}$ spectra of compounds $3-3 \mathrm{a}, 3-4 \mathrm{~h}$ and $3-5 \mathrm{~h}$.}

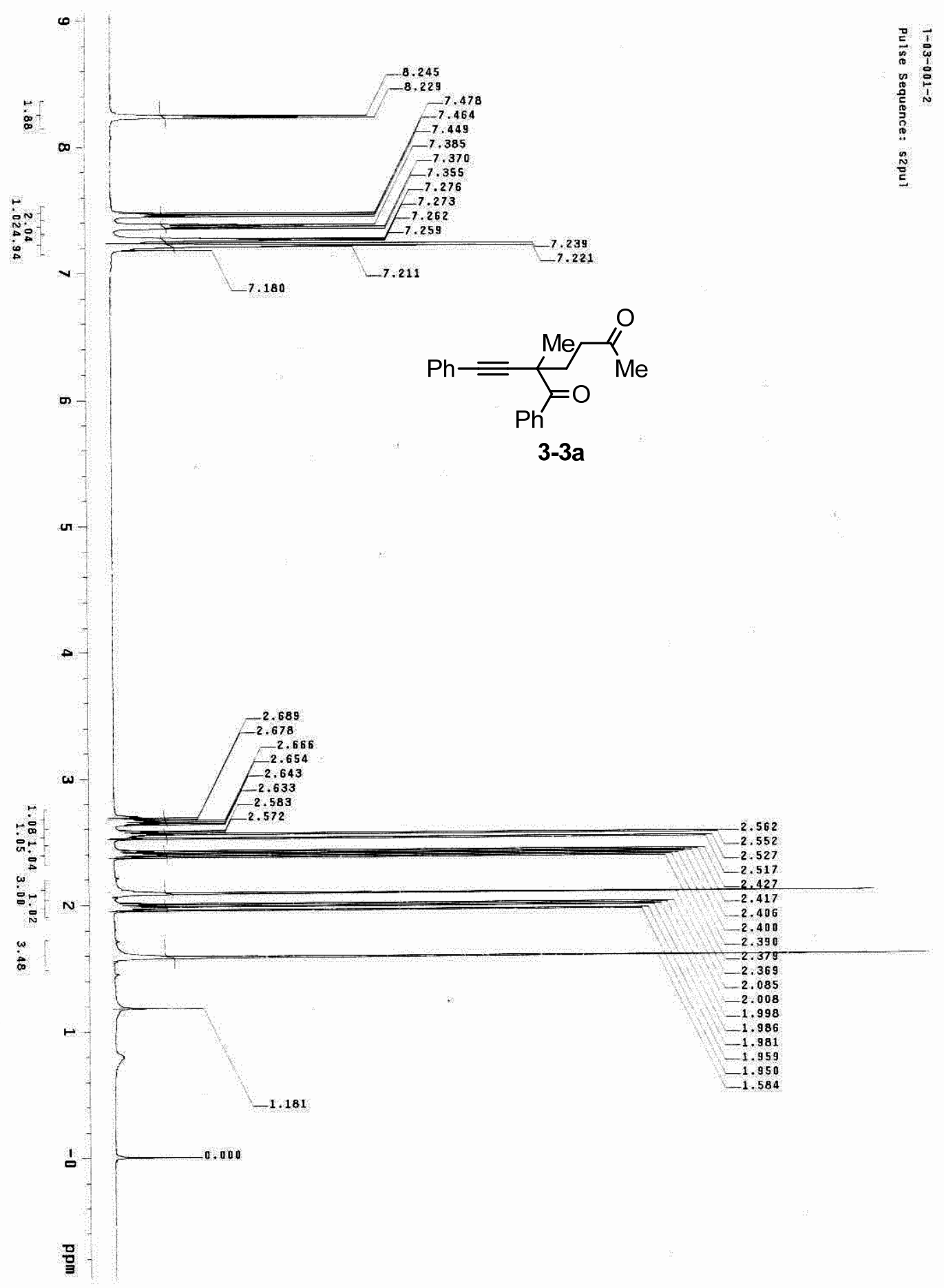




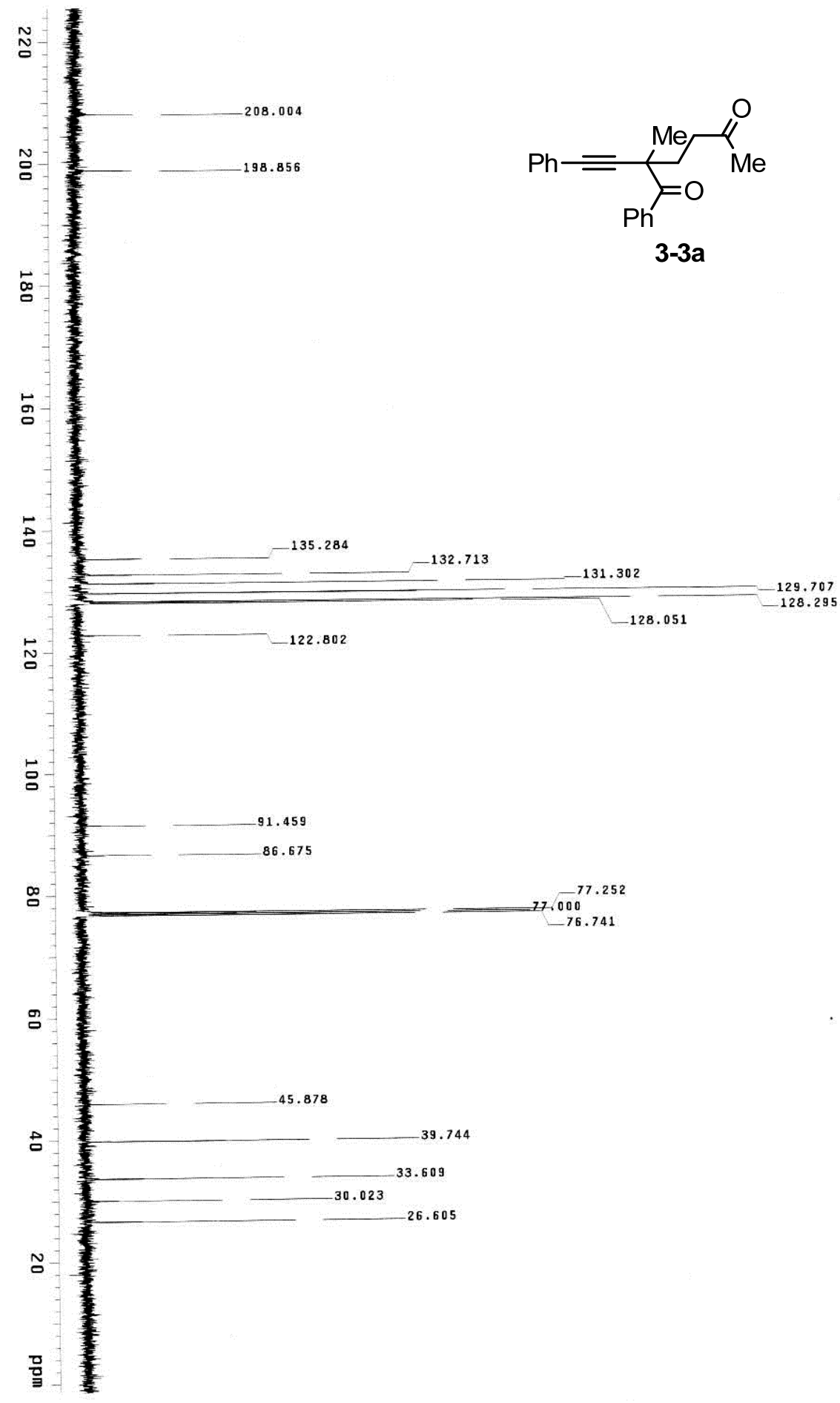




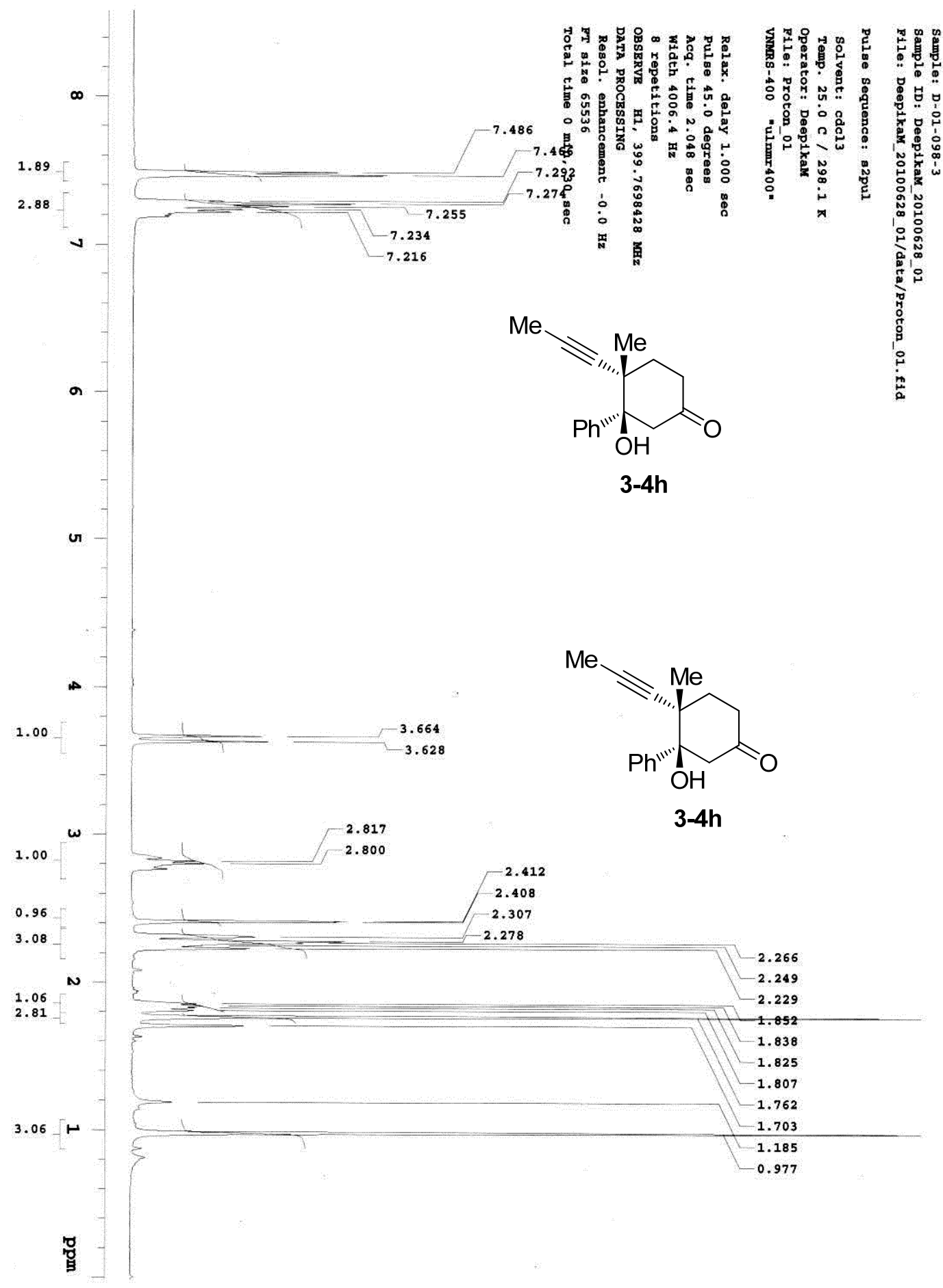




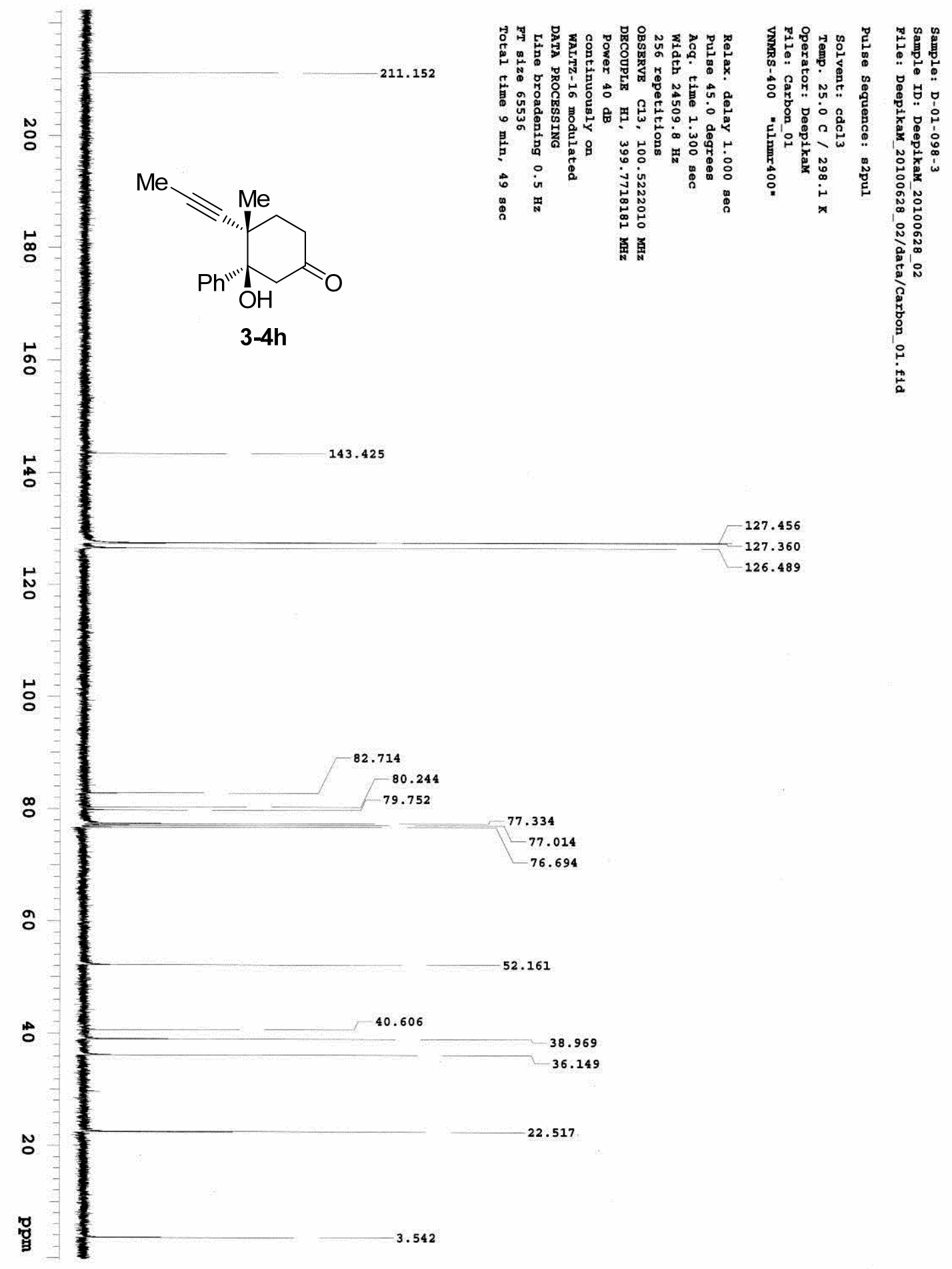




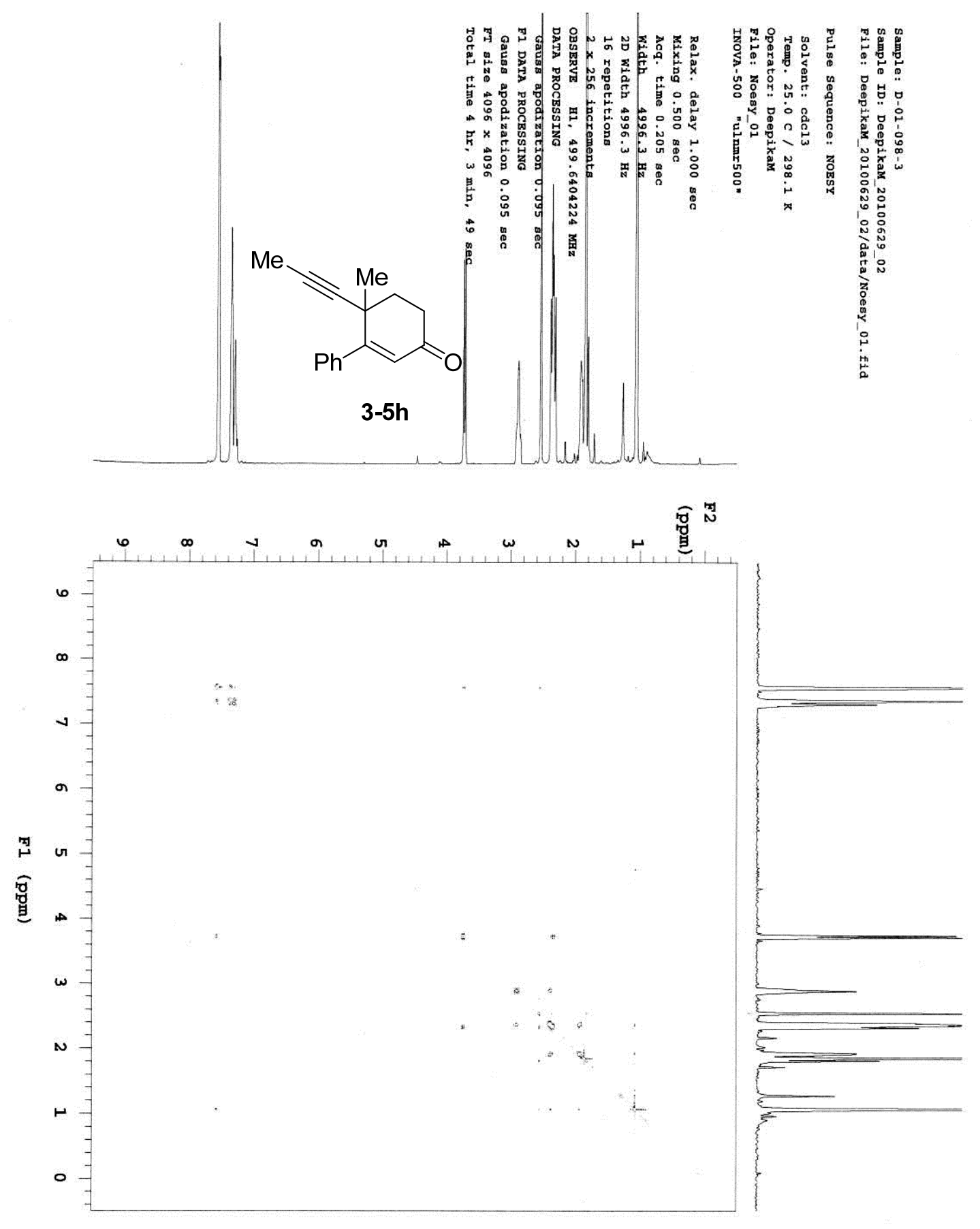




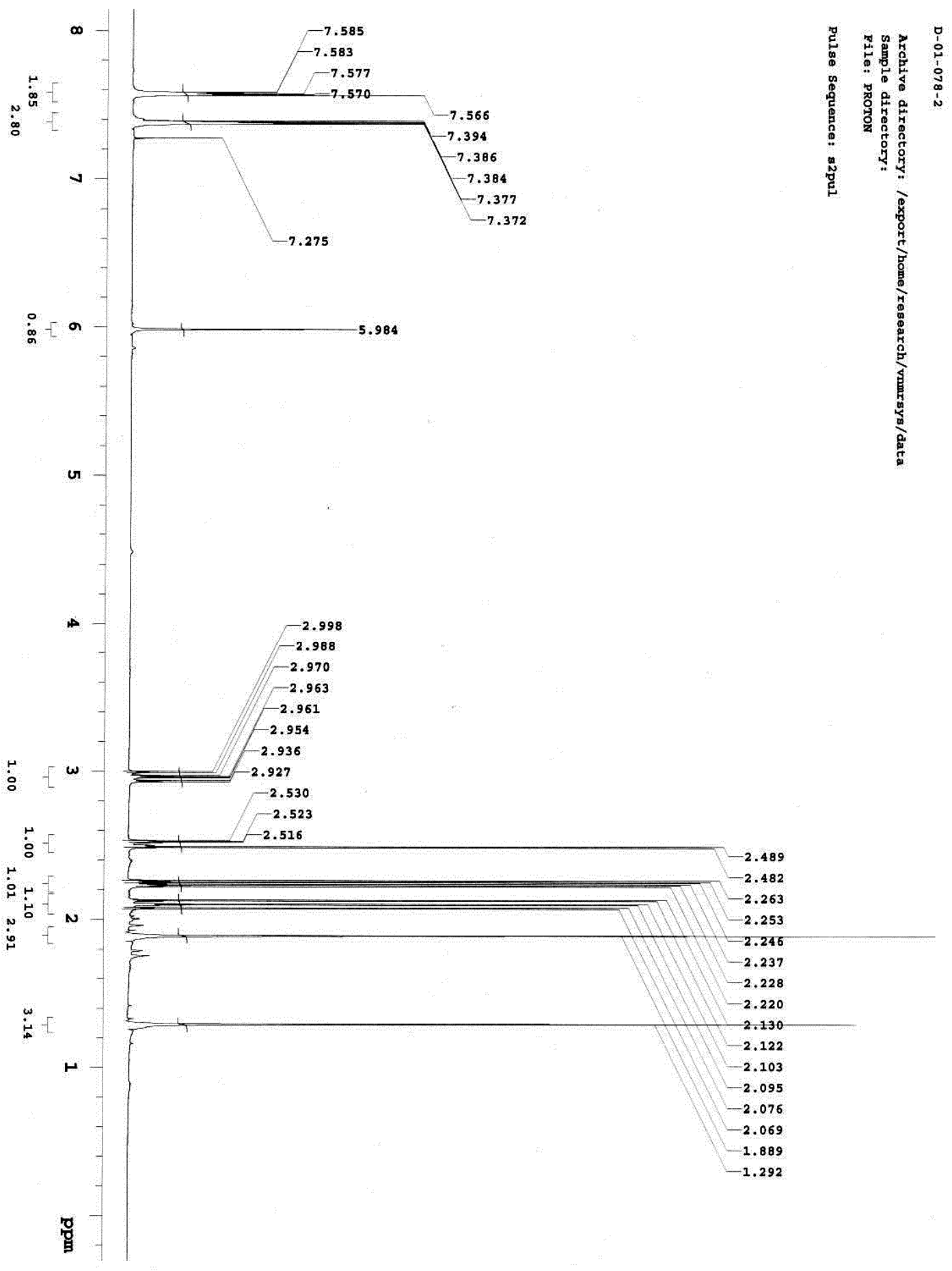




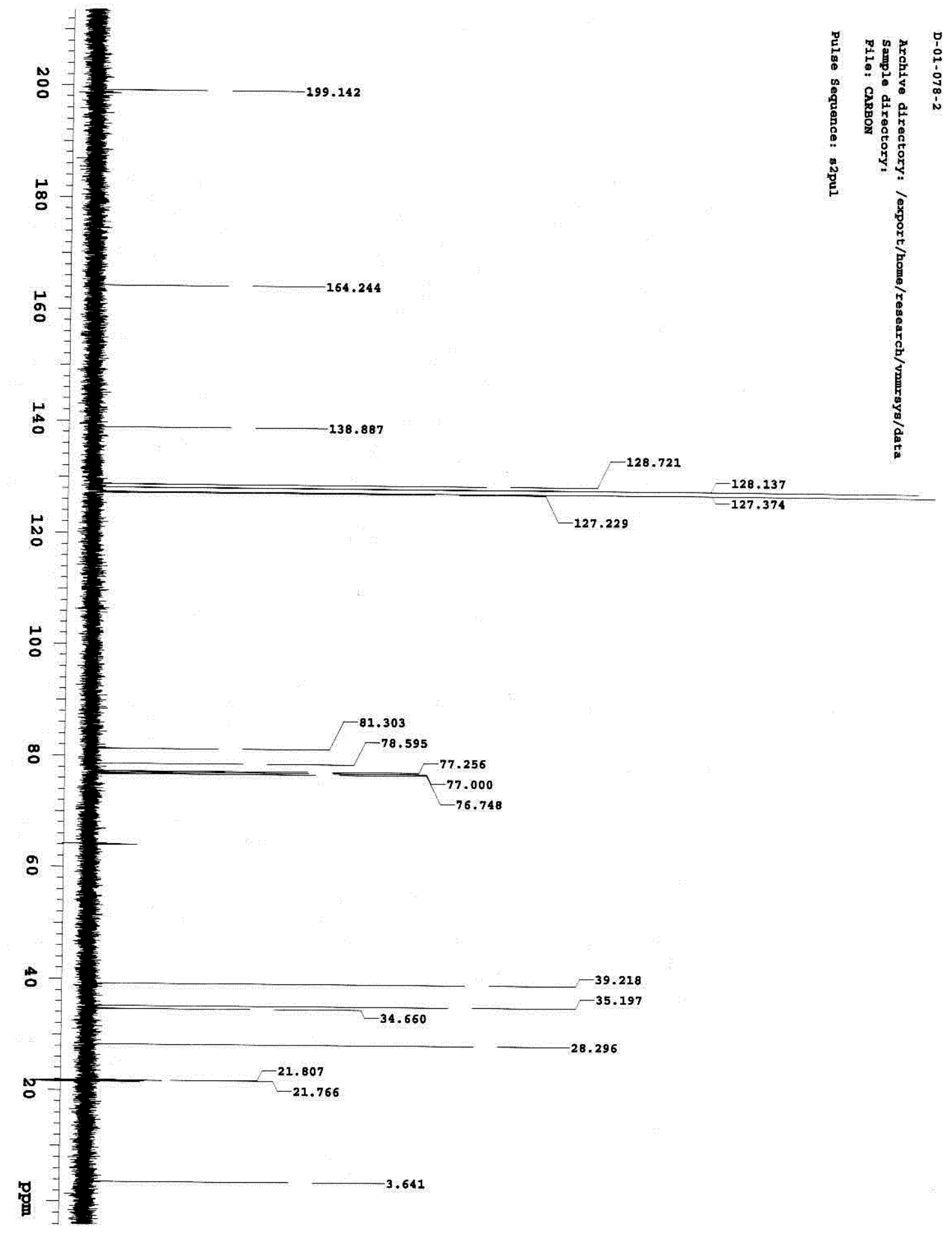




\section{CHAPTER 4. GOLD-CATALYZED ANNULATIONS OF 2-ALKYNYL BENZALDEHYDES}

\subsection{Background}

Gold-catalyzed transformations have been used extensively in the field of synthetic organic chemistry to rapidly and effectively construct interesting organic frameworks. ${ }^{1,20,149,213-218}$ The development of gold catalysts with improved catalytic activity and stability has also attracted interest. ${ }^{7,18,88,219-222}$ Selected recent notable examples include N,O-chelating gold(III) catalysts, ${ }^{2,3,223-225} \mathrm{~N}$-heterocyclic carbene (NHC) or acyclic carbene gold catalysts, ${ }^{226-241}$ cationic gold acetonitrile complexes ${ }^{242-}$ 244 containing Buchwald-type phosphine ligands ${ }^{245-250}$ and cationic gold-triazole complexes. ${ }^{251-257}$ On the other hand, counteranions, such as super acid anions including the bis(trifluoromethanesulfonyl)imide $\left(\mathrm{NTf}_{2}\right),{ }^{258-262}$ phosphates, ${ }^{263-269}$ and even hydroxide, ${ }^{19,270,271}$ have been found to play a significant role in the catalytic activities of gold catalysts (Scheme 29).

Due to their easy activation by various transition-metal catalysts and Lewis acids, the readily available 2-alkynyl benzaldehyde 4-1 has served as a versatile scaffold in a number of organic transformations ${ }^{107,272-278}$ amongst which gold-catalyzed annulations stand out. ${ }^{95,97,279-283}$ Notably, Yamamoto and co-workers reported a gold-catalyzed benzannulation of 2-alkynyl benzaldehyde with an alkyne thereby furnishing naphthalene 
derivative 4-2 in a highly efficient manner. ${ }^{22} \mathrm{~A}$ formal Diels-Alder-type [4+2]cycloaddition mechanism for this gold-catalyzed annulation was proposed, but a stepwise mechanism via intermediate $\mathbf{B}$ was also suggested and supported by the substituent effects. However, decarbonylation occurred to yield 4-3 as the main product when copper was used as catalyst. ${ }^{93}$
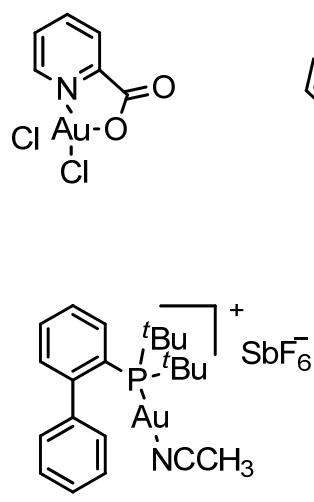

Au-1

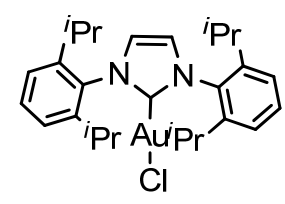

(IPr)AuCl

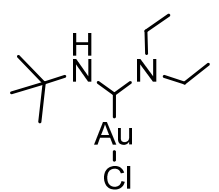

$\mathrm{Cl}$

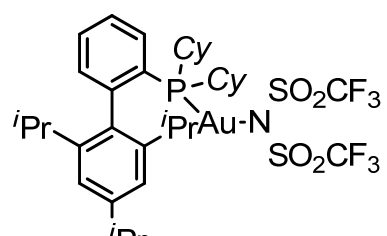

Au-2

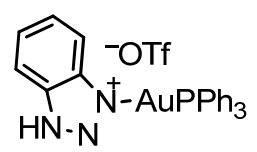

Triazole-Au

Scheme 29: Selected examples of recently developed gold catalysts.

The group of Yamamoto extended their formal [4+2]-cycloaddition protocol to alkenes, obtaining dihydronaphthalene derivative $\mathbf{4 - 4}$, but in this case the copper catalyst exhibited better catalytic activity than gold. ${ }^{94}$ Carbocation intermediate $\mathbf{B}$ was called for to account for the observed regio- and stereoselectivities. However, when vinyl ether was employed in the annulation with 2-alkynyl benzaldehyde, the reaction yielded nathphalene 4-2, rather than dihydronaphthalene 4-4. This result has been ascribed to the elimination of alcohol. This outcome was corroborated by work reported by the Porco group. ${ }^{122}$ Aldehydes or acetals having $\alpha$-protons have been effectively involved in this gold-catalyzed annulation, but these substrates served as an enol source instead of a carbonyl source, affording the naphthalene product 4-2, due to the elimination of a water molecule (Scheme 30). ${ }^{36}$ 


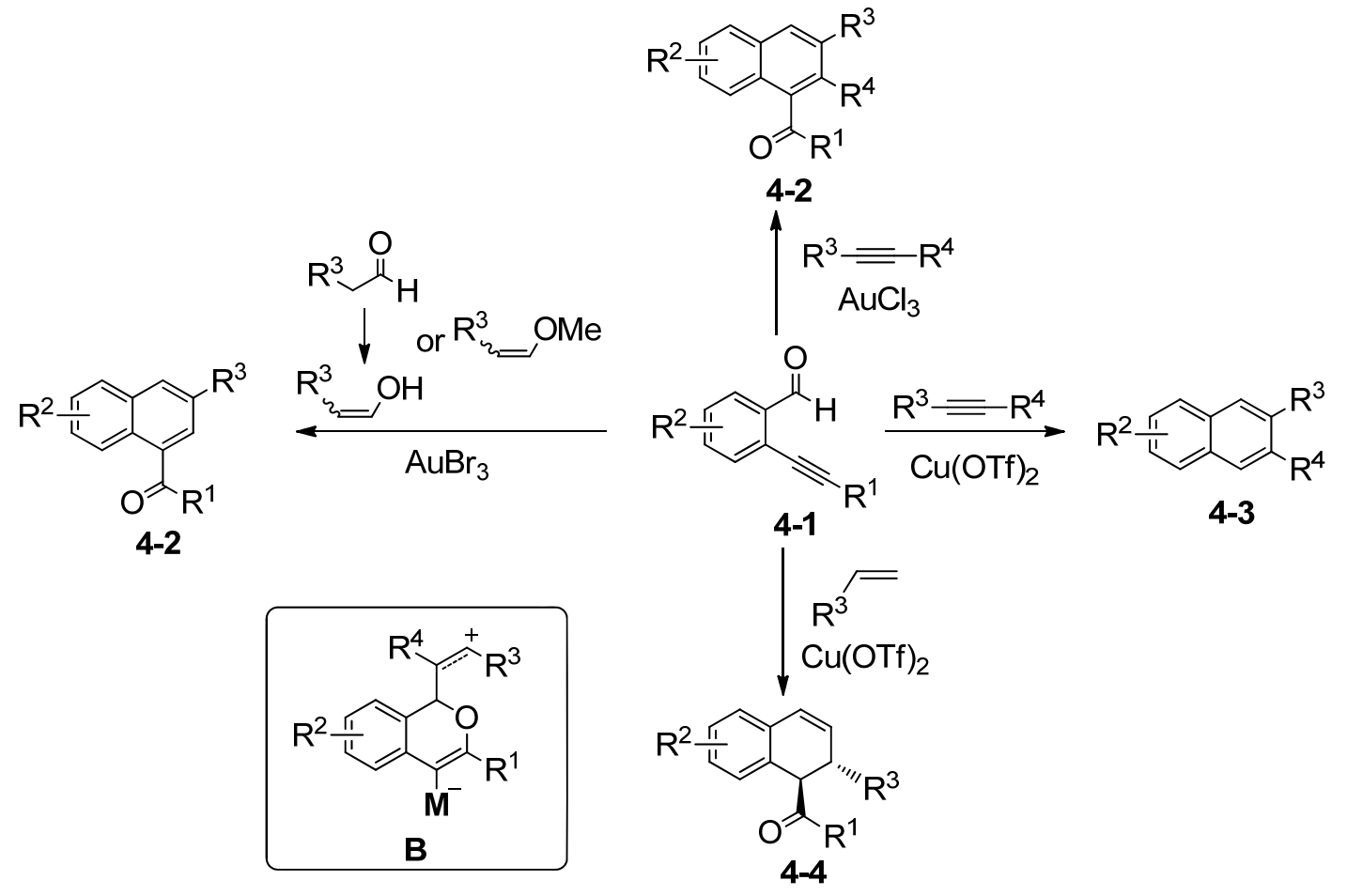

Scheme 30: Yamamoto's group pioneered the gold or copper-catalyzed annulation of 2-alkynyl benzaldehyde with alkyne, alkene, vinyl ether, or aldehyde. $R_{4}$ is $H$ or alkyl in $B$.

In line with our continuing interest in gold catalysis and ligand effects, ${ }^{41,46,87,127,284-287}$ we posited that a gold catalyst containing a suitable ligand may help to stabilize the carbocation intermediate and veer the reaction toward new synthetic paths. Recently, we reported the gold-catalyzed intramolecular annulations of 2-(ynol)aryl aldehydes 4-10, in which different gold catalysts furnished benzochromanes 4-11 or benzobicyclo[4.3.1] acetals 4-12 (Scheme 31). ${ }^{128}$ Thus, we envisioned that the reaction of 2-alkynyl benzaldehyde with a vinyl ether might yield a synthetically interesting product if a suitable gold catalyst could be found. Interestingly, we found that by selecting Au$\mathbf{1}$ as the catalyst, 2-alkynyl benzaldehyde reacted with vinyl ether to form acetal-tethered dihydronaphthalene 4-5 and isochromene 4-6, rather than naphthalene 4-2. Furthermore, a symmetrical bicyclo[2.2.2] octane 4-7 was produced from the reaction of 2-alkynyl benzaldehyde with a cyclic vinyl ether. By using (IPr)Au as the catalyst, a homo- 
dimerization of 2-alkynyl benzaldehyde took place, affording a set of separable diastereomers 4-9 (Scheme 31). These interesting cyclic products are potentially useful synthetic templates of biologically active molecules and natural products such as codeine, ${ }^{288} \mathrm{CJ}-17493,{ }^{289}$ psychorubrin, ${ }^{290}$ trioxifene, ${ }^{291}$ regalamine, robustamine, ${ }^{292}$ and the trypticene analogue TT13 $3^{293,294}$ (Scheme 32). To the best of our knowledge, the synthesis of similar cyclic compounds is rare, and, normally, harsh reaction conditions are needed. ${ }^{295-302}$

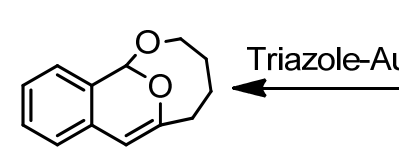

4-12<smiles>[R]CC1CC2CCCC2C1C([R])C(=O)O</smiles>

4-9<smiles>O=Cc1ccccc1C#CCCCCCO</smiles>

4-10

4-1

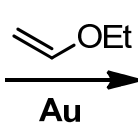<smiles>[R]C#Cc1ccccc1C=O</smiles>

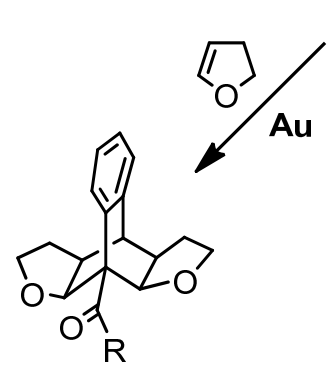

4-7<smiles>c1ccc2cc3c(cc2c1)CCCO3</smiles>

4-11<smiles>[R]C(=O)C1=CCC(CC(OCC)OCC)c2ccccc21</smiles>

Scheme 31: New developments on gold-catalyzed annulations of 2-alkynyl benzaldehydes with vinyl ethers. 
<smiles>COc1cc2c(cc1CN[C@@H]1CCCN[C@H]1c1ccccc1)[C@@](C)(C(F)(F)F)OCC2</smiles>

CJ-17493

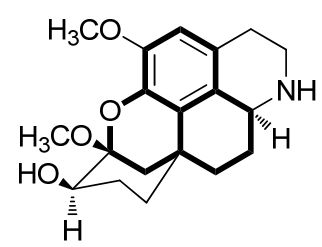

Robustamine<smiles>O=C(C1=C(c2ccc(O)cc2)CCc2ccccc21)c1ccc(OCCN2CCCC2)cc1</smiles>

Trioxifene

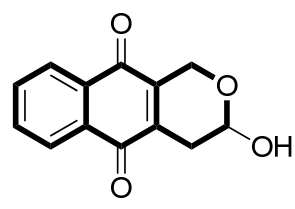

Psychorubrin<smiles>COc1ccc2c3c1O[C@H]1[C@H](O)C=C[C@]3(C)[C@H]1CCN2C</smiles>

Codeine<smiles>COC1=C(Br)C(=O)C2=C(C1=O)C1C3C=CC=CC3C2C2C(=O)C=CC(=O)C12</smiles>

TT13

Scheme 32: Selected examples of biologically active compounds containing dihydronaphthalene, isochromene, or bicyclo[2.2.2]octane substructures.

\subsection{Results and discussion}

Although for the most part the selection of gold catalysts relies on an empirical trial and error approach, some patterns on ligand effect in gold catalysis have emerged recently. ${ }^{41,284,285}$ For example, the cationic gold complex Au-1, developed by Echavarren and co-workers, exhibited excellent stability and catalytic activity in the cycloisomerization of enynes due to its bulky and electron-rich phosphine ligand. ${ }^{176,215,303,304}$ Accordingly, we first employed Au-1 as the catalyst in the reaction of 2-phenylethynyl benzaldehyde with ethyl vinyl ether. To our delight, along with Yamamoto's naphthalene product 4-2a (which was obtained in low yield) we isolated the acetal-tethered dihydronaphthalene derivative 4-5a as the major product, under mild conditions (Table 6, entry 1). Other catalysts such as $\mathrm{AuCl}, \mathrm{AuCl}_{3}$, triazole gold, and (IPr)AuCl/AgOTf, as well as AgOTf failed to give any identifiable product. The fragility of this reaction may be due to the instability of ethyl vinyl ether to acidic 
conditions. ${ }^{305,306} \mathrm{Next}$, various 2-alkynyl benzaldehyde substrates with different substituents on the aromatic ring were prepared for use in this new gold-catalyzed annulation with vinyl ether, and the results are summarized in Table 6. All the reactions gave the desired dihydronaphthalene derivatives in moderate yields at, or below, room temperature. Mechanistically, the tethered acetal might have been generated from an oxonium intermediate reacting with an alkoxide anion. ${ }^{126}$ Thus, we wondered if the oxonium intermediate could be intramolecularly trapped by an adjacent phenolic hydroxyl group or if the desired product would be formed preferably in the presence of additional ethanol. To our satisfaction, when substrate 4-1f was employed in this reaction, the reaction intermediate was indeed trapped by the adjacent phenolic hydroxyl group, furnishing the structurally interesting tricyclic compound $\mathbf{4 - 5 f}$ as the main product (Table 6, entry 6) ${ }^{307}$ However, when two equivalents of ethanol were added to the reaction, a new acetal-tethered isochromene 4-6a was obtained in good yield, rather than the corresponding dihydronaphthalene derivative (Table 7, entry 1). 
Table 6: Scope of the gold-catalyzed annulation of 2-alkynyl benzaldehyde with vinyl ether. ${ }^{\text {a }}$

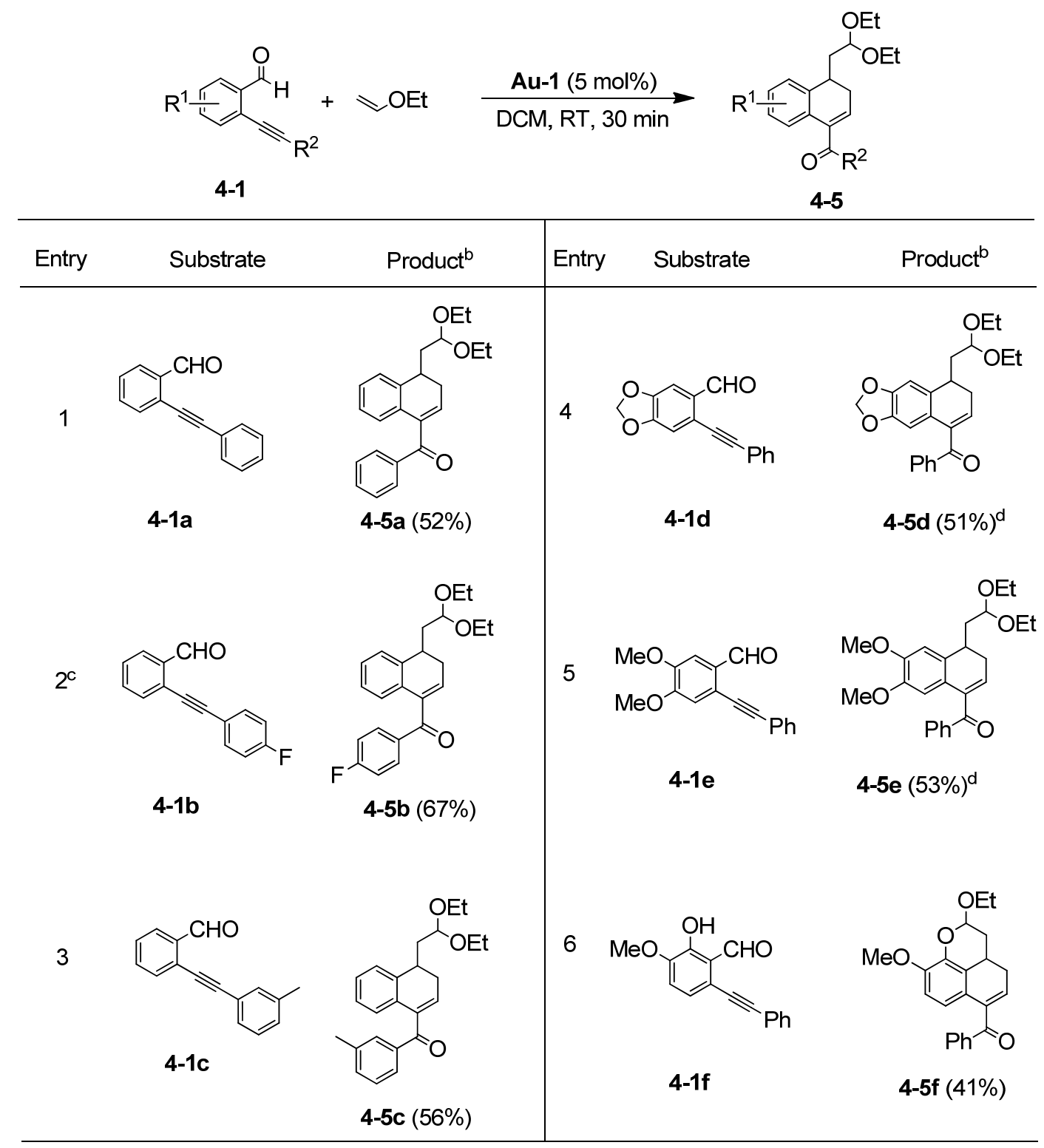

[a] General reaction conditions: 2-alkynyl benzaldehyde 4-1 $(0.145 \mathrm{mmol})$, ethyl vinyl ether $(0.72 \mathrm{mmol})$, Au-1 (5 mol\%), $\mathrm{CH}_{2} \mathrm{Cl}_{2}(1.0 \mathrm{~mL})$; [b] isolated yields; [c] reaction time: $15 \mathrm{~min}$; [d] ethyl vinyl ether (1.45 $\mathrm{mmol}$ ) was used and the reaction was carried out at $0{ }^{\circ} \mathrm{C}$. 
Table 7: Gold-catalyzed annulation of 2-alkynyl benzaldehyde with vinyl ether in the presence of alcohol. ${ }^{\mathrm{a}}$

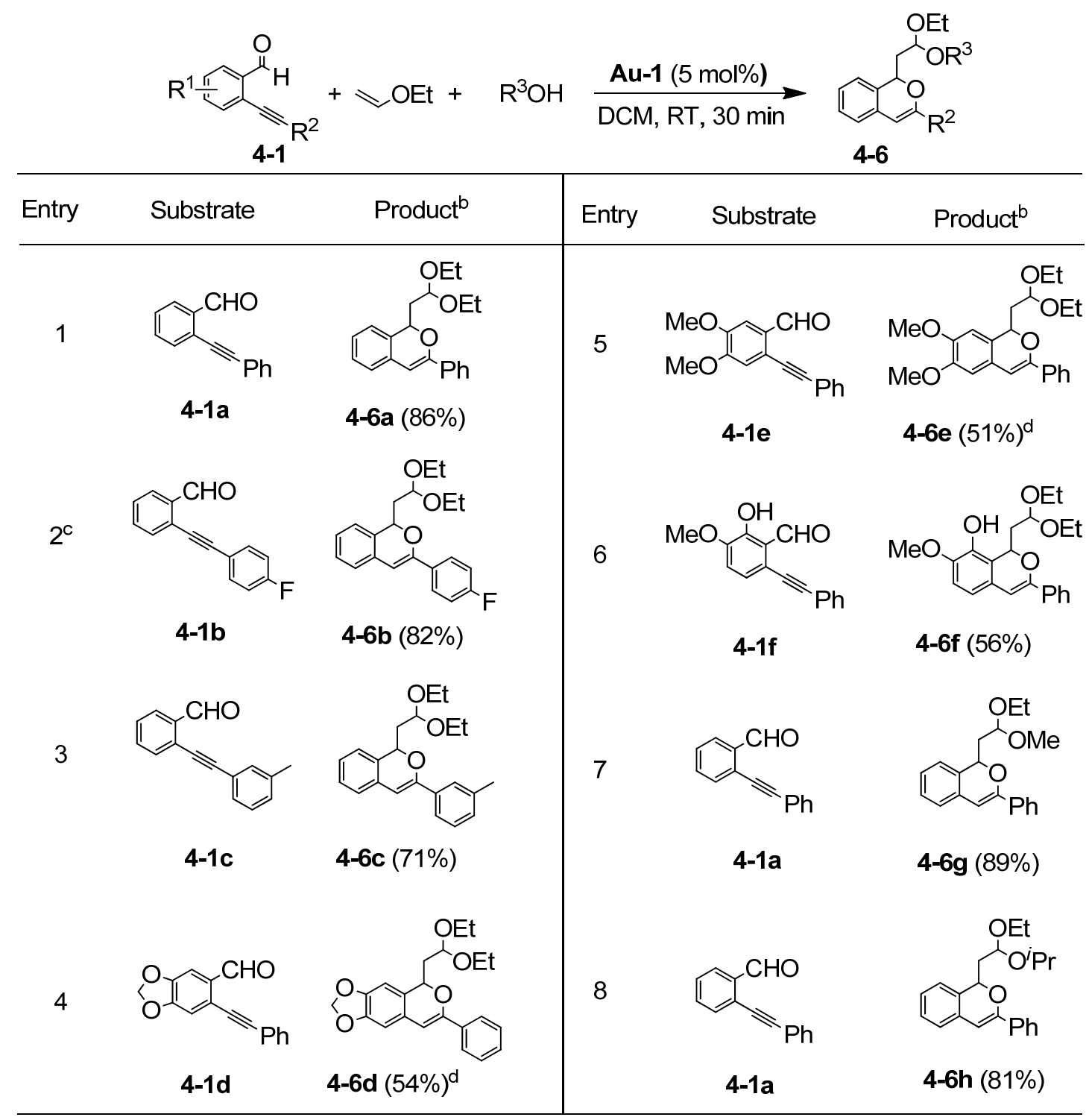

[a] General reaction conditions: 2-alkynyl benzaldehyde 4-1 $(0.145 \mathrm{mmol})$, ethyl vinyl ether $(0.72 \mathrm{mmol})$, alcohol (0.29 mmol), Au-1 (5 mol\%), $\mathrm{CH}_{2} \mathrm{Cl}_{2}(1.0 \mathrm{~mL})$; [b] isolated yields; [c] reaction time: $10 \mathrm{~min}$; [d] reaction was carried out at $0{ }^{\circ} \mathrm{C}$.

Since isochromene derivatives are also attractive substrates in organic chemistry, we investigated the gold-catalyzed annulation of 2-alkynyl benzaldehydes with vinyl ether in the presence of alcohol. The results are outlined in Table 7. All the desired products were obtained in good yields. It should be noted that, for substrate 4-1f, the intramolecular trapping was bypassed by an intermolecular quenching with ethanol (Table 7, entry 6), 
which could be explained by the fact that alkoxide is a stronger nucleophile than phenoxide. Other alcohols, such as methanol and isopropanol, were tested in this reaction, and similar products were obtained in good yields (Table 7, entries 7 and 8).

Plausible mechanisms for the formation of these new products have been proposed, as outlined in Scheme 33. The generation of intermediate $\mathbf{A}$ from a gold catalyst and 2alkynyl benzaldehyde substrate is well-accepted, ${ }^{100,106,110,113-116,118,119}$ whereas the subsequent annulation with vinyl ether to form $\mathbf{C}$ is not clear. It could be either a concerted step, as in a Diels-Alder-type reaction, or in stepwise fashion, beginning with the formation of $\mathbf{B}$. According to previous literature reports, the existence of $\mathbf{B}$ is supported by substituent effects and regio- and stereoselectivities. ${ }^{22,36,93,94,122}$ In our case, the formation of acetal-tethered isochromene 4-6 in the presence of alcohol also implies the generation of intermediate $\mathbf{B}$. However, intermediate $\mathbf{A}$ was not trapped by alcohol before the formation of $\mathbf{B}$, demonstrating the different catalytic activity of the gold catalyst. ${ }^{308-312}$ Once intermediate $\mathbf{C}$ was formed, it could transform into $\mathbf{D}$, which reacted with another molecule of vinyl ether, generating intermediate $\mathbf{E}$. Gold and ethoxide elimination and quenching of the oxonium ion with ethoxide took place in $\mathbf{E}$, affording 4$\mathbf{5}$ as the product and re-generating the gold catalyst. ${ }^{313,305,306} \mathrm{~A}$ competitive elimination in $\mathbf{D}$ also occurred, furnishing naphthalene 4-2 as the product. With the selection of an appropriate gold catalyst, the elimination could be controlled. 


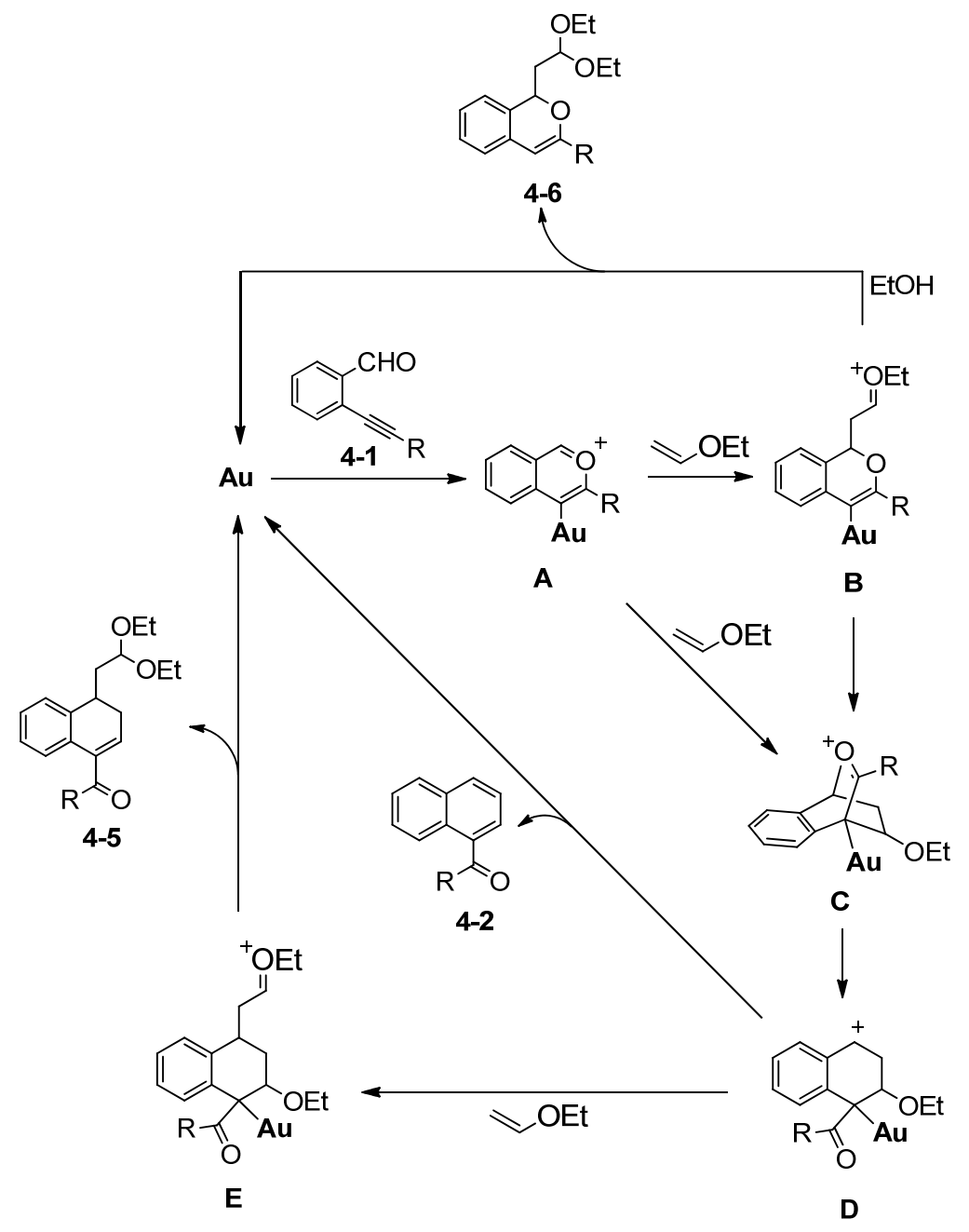

Scheme 33: Proposed mechanisms for the gold-catalyzed annulations of 2-alkynyl benzaldehyde with vinyl ether.

Because gold alkoxide elimination took place at the stage of intermediate $\mathbf{E}$, we envisioned that this elimination might have been prevented if a cyclic vinyl ether was chosen as the annulation partner. Thus, the readily available 2,3-dihydrofuran was employed in the reaction, and as expected, the gold alkoxide elimination was successfully prevented. Instead, a new bicyclo[2.2.2] octane derivative 4-7 was produced, in which two molecules of cyclic vinyl ether were involved. Catalyst $\mathbf{A u - 2} \mathbf{2}^{314}$ showed the best catalytic activity and therefore it was selected for subsequent reactions. 
Table 8: Gold-catalyzed annulation of 2-alkynyl benzaldehyde with cyclic vinyl ether. ${ }^{a}$

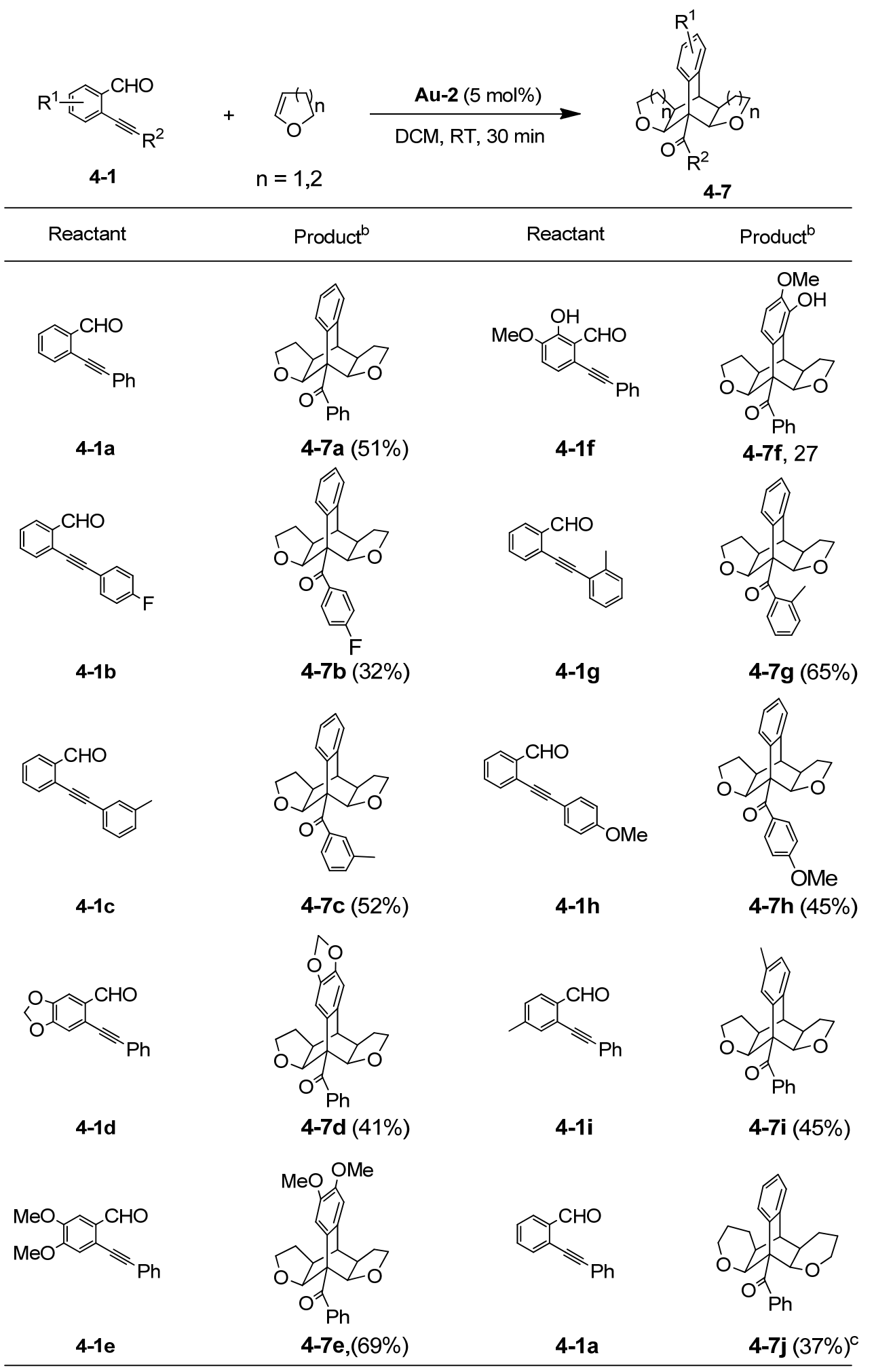

[a] General reaction conditions: 2-alkynyl benzaldehyde 4-1 $(0.145 \mathrm{mmol}), 2,3$-dihydrofuran $(0.72 \mathrm{mmol})$, Au-2 (5 mol\%), $\mathrm{CH}_{2} \mathrm{Cl}_{2}$ (1.0 mL); [b] isolated yields in \%; [c] 3,4-dihydro-2H-pyran was used as cyclic vinyl ether. 
Various substrates, having different substituents on the aromatic rings, were employed in this new gold-catalyzed annulation with 2,3-dihydrofuran, and the corresponding products 4-7a-i were isolated (Table 8). 3,4-Dihydro-2H-pyran was also employed as the cyclic vinyl ether due to its easy availability, and the corresponding product $\mathbf{4 - 7} \mathbf{j}$ was obtained in moderate yield.

The structure of product 4-7a was determined by using ${ }^{1} \mathrm{H},{ }^{13} \mathrm{C}$, DEPT, gCOSY, HMQC, and HMBC NMR spectroscopy as well as HRMS, and finally confirmed by using X-ray crystallography (the ORTEP-3 diagram of 4-7a is shown in Figure 8).

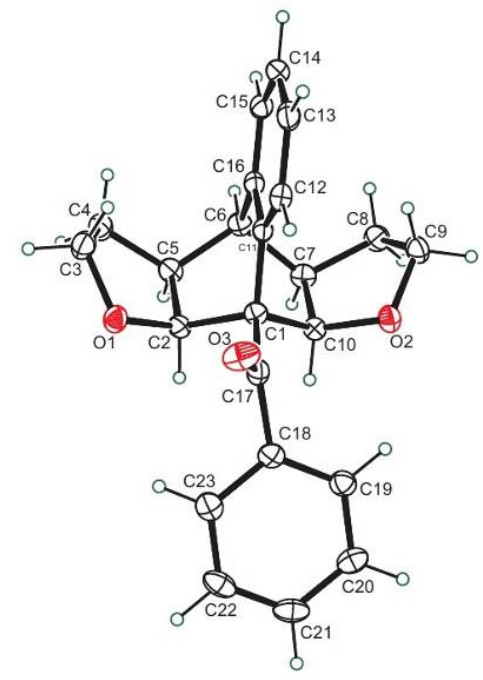

Figure 8: ORTEP-3 diagram of bicyclo[2.2.2]octane derivative 4-7a, showing $40 \%$ probability ellipsoids. $H$ atoms are shown as small spheres of arbitrary radii. Selected bond lengths $[\AA]]$ and angles [ ${ }^{\circ}$ ]: O1-C2, 1.4285(17); O1-C3, 1.4380(18); O2-C9, 1.4419(18); O2-C10, 1.4338(16); O3-C17, 1.2100(18); O2-C10-C1, 111.15(11); O1-C2-C3, 111.78(11); C2-C1-C10, 104.06(11).

A plausible mechanism for the formation of the bicyclo[2.2.2]octane derivative is outlined in Scheme 34. After intermediate $\mathbf{A}$ is generated, a formal [4+2] cycloaddition to 2,3-dihydrofuran could take place either via a concerted or stepwise fashion to yield intermediate $\mathbf{G}$. Intermediate $\mathbf{G}$ could transform itself into $\mathbf{H}$, which then reacts with a second molecule of 2,3-dihydrofuran, generating I, before finally furnishing product 4- 
7 and regenerating the gold catalyst. However, an alternative possibility could also be taken into account. Elimination of gold catalyst from $\mathbf{H}$ could generate tetrahydro naphthofuran derivative 4-8, which would then undergo an inverse-electron-demand Diels-Alder reaction with the second molecule of 2,3-dihydrofuran, furnishing the final product $4-7 .{ }^{36}$

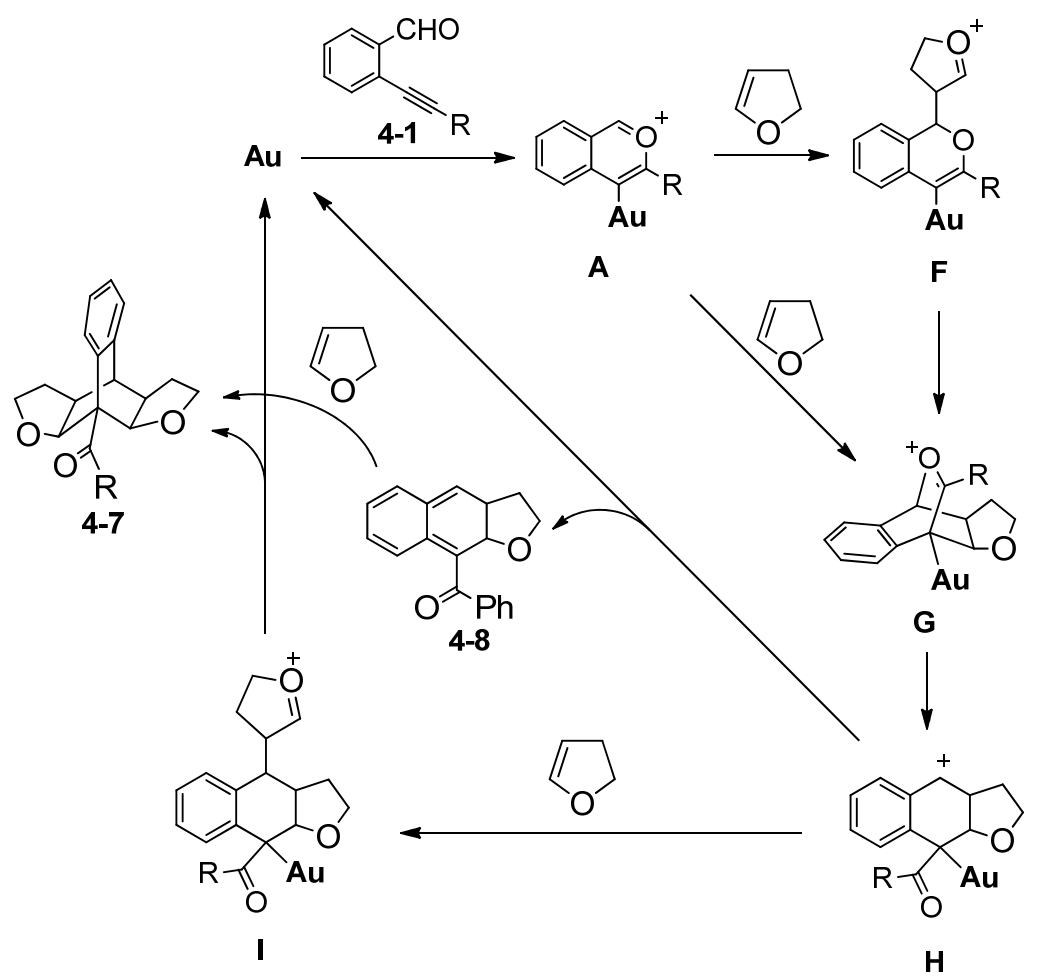

Scheme 34: Proposed mechanism for the gold-catalyzed annulation of 2-alkynyl benzaldehyde with cyclic vinyl ether.

During our investigations on these gold-catalyzed annulations, homo-dimerization of 2alkynyl benzaldehyde was observed when (IPr)AuCl/AgOTf was employed as the catalyst in the absence of vinyl ether (Scheme 35$).{ }^{16} \mathrm{~A}$ set of separable diastereoisomers 4-9a and 4-9b was obtained in good yield, in a 3:5 ratio. The formation of this dimer has been ascribed to a gold-catalyzed hydrolysis of the 2-alkynyl benzaldehyde substrate, generating ketone 4-10, which then serves as a nucleophile for 
attacking the electrophilic intermediate A. The structure of the dimer was also confirmed by X-ray crystallography. The ORTEP-3 diagram of the anti-isomer 4-9b is shown in Figure 9.

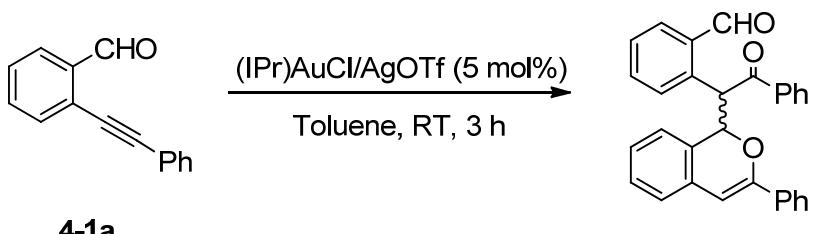

4-1a

4-9, $84 \%$

$[\operatorname{syn}(4-9 a): a n t i(4-9 b)=3: 5]$<smiles>O=Cc1ccccc1C#Cc1ccccc1</smiles>

4-1

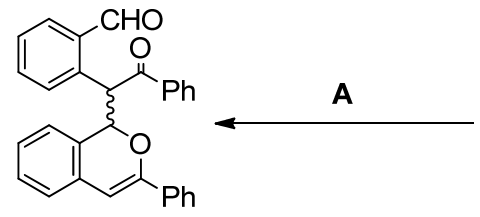

4-9

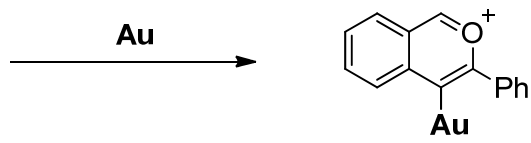

A
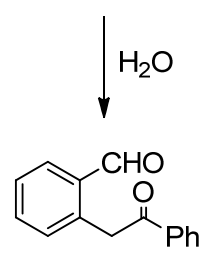

4-10

Scheme 35: Gold-catalyzed homo-dimerization of 2-alkynyl benzaldehyde and the proposed mechanism.

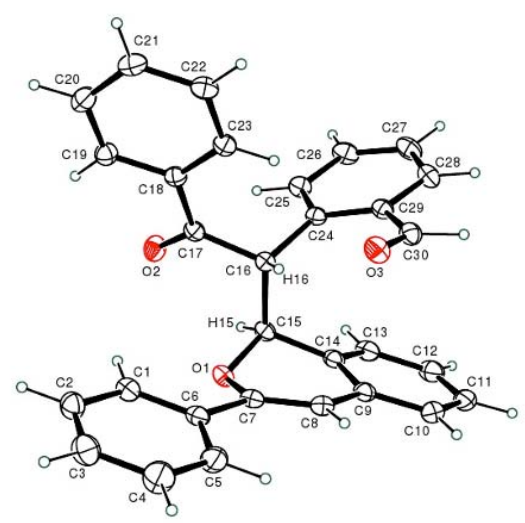

Figure 9: ORTEP-3 diagram of product 9 b, showing one diastereoisomer of the racemic mixture at $40 \%$ probability ellipsoids. $H$ atoms are shown as small spheres of arbitrary radii. Selected bond lengths $[\AA ̊]$ and angles $\left[^{\circ}\right]:$ O1-C7, 1.3795(15); O1-C15, 1.4447(15); O2-C17, 1.2157(15); O3-C30, 1.2117(16); C15-C16, 1.5531(17); C7-O1-C15, 114.70(9); O1-C7-C8, 121.07(11); O1-C15-C16, 106.72(9). 


\subsection{Conclusions}

We have investigated the gold-catalyzed annulations of 2-alkynyl benzaldehyde with acyclic or cyclic vinyl ethers under very mild conditions, and found a number of synthetically interesting transformations. With the selection of an appropriate gold catalyst, the reactions of various 2-alkynyl benzaldehydes with acyclic vinyl ethers afforded dihydronaphthalene derivatives as the products, whereas acetal-tethered isochromenes were obtained when the reaction was conducted in the presence of alcohol. Interestingly, a new bicyclo[2.2.2] octane derivative was isolated from the reaction of 2alkynyl benzaldehyde with cyclic vinyl ether. The various mechanisms of formation of these reactions have been discussed. We also found that 2-alkynyl benzaldehyde undergoes dimerization under gold catalysis in the absence of vinyl ether. The work described in this chapter was published in Chem. Eur. J. 2013, 19, 4043-4050.

\subsection{Experimental}

\section{General}

${ }^{1} \mathrm{H},{ }^{13} \mathrm{C}$ and ${ }^{19} \mathrm{~F}$ NMR spectra were recorded at 500, 126 and 470 (or 400, 101 and 376) $\mathrm{MHz}$, respectively, by using $\mathrm{CDCl}_{3}$ as a solvent. The chemical shifts are reported in $\delta(\mathrm{ppm})$ values $\left({ }^{1} \mathrm{H}\right.$ and ${ }^{13} \mathrm{C}$ NMR relative to $\mathrm{CHCl}_{3}, \delta=7.26 \mathrm{ppm}$ for ${ }^{1} \mathrm{H} \mathrm{NMR}$ and $\delta=$ $77.0 \mathrm{ppm}$ for ${ }^{13} \mathrm{C}$ NMR and $\mathrm{CFCl}_{3}\left(\delta=0 \mathrm{ppm}\right.$ for ${ }^{19} \mathrm{~F}$ NMR $)$, multiplicities are indicated by s (singlet), d (doublet), t (triplet), q (quartet), p (pentet), h (hextet), $\mathrm{m}$ (multiplet) and br (broad). Coupling constants, $J$, are reported in Hertz (Hz). Solvents (tetrahydrofuran, ether, dichloromethane and DMF) were dried using a commercial solvent purification system. All other reagents and solvents were employed without further purification. The 
products were purified using a commercial flash chromatography system or a regular glass column. TLC was developed on silica gel 60 F254 aluminum sheets. High resolution ESI-MS were obtained using a MS-FTICR-MS ${ }^{\mathrm{n}}$ system (LTQ FT, Thermo Electron Corp.) at the CREAM Mass Spectrometry Facility, University of Louisville, Kentucky.

\section{General procedure for the formation of dihydronaphthalenes (4-5):}

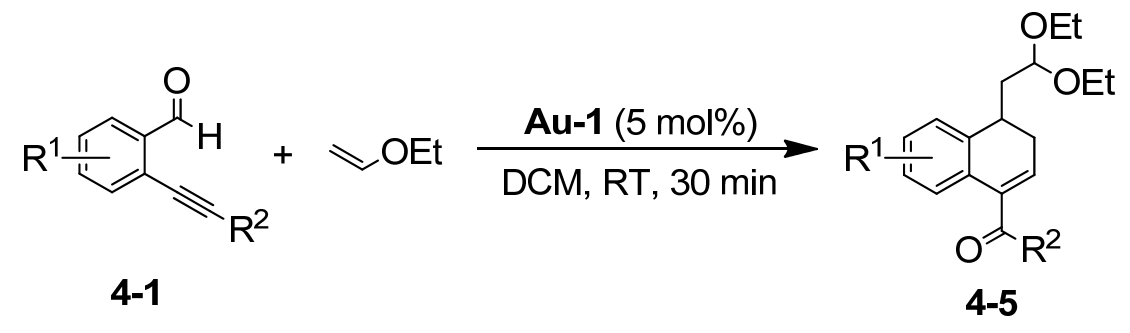

Au-1 (0.0072 mmol) was added to a solution of 2-alkynylbenzaldehyde 4-1 $(0.145 \mathrm{mmol})$, ethyl vinyl ether 4-2a $(0.72 \mathrm{mmol})$ in DCM $(1.0 \mathrm{~mL})$. The mixture was stirred for $30 \mathrm{~min}$ at room temperature. The reaction mixture was quenched with water and extracted with DCM. The solvent in the organic layer was removed under reduced pressure and the residue was subjected to flash column chromatography (eluent: ethyl acetate/n-hexane = 1/10) to give product $4-5$ as a colorless oil.

\section{Spectroscopic data of compounds 4-5}

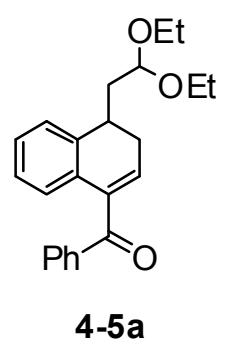


Data for 4-5a : colorless oil; ${ }^{1} \mathrm{H}$ NMR $\left(\mathrm{CDCl}_{3}, 400 \mathrm{MHz}\right) \delta 1.13-1.16(3 \mathrm{H}, \mathrm{t}, J=7.2 \mathrm{~Hz})$, 1.23-1.26 (3H, t, $J=7.2 \mathrm{~Hz}), 1.84-1.88(1 \mathrm{H}, \mathrm{m}), 1.93-2.0(1 \mathrm{H}, \mathrm{m}), 2.42-2.49(1 \mathrm{H}, \mathrm{m})$, 2.68-2.74 (1H, m), 3.01-3.06 (1H, m), 3.39-3.59 (3H, m), 3.63-3.71 (1H, m), 4.44-4.47 $(1 \mathrm{H}, \mathrm{t}, J=6.4 \mathrm{~Hz}), 6.39-6.42(1 \mathrm{H}, \mathrm{dd}, J=6.4,3.2 \mathrm{~Hz}), 7.12-7.17(1 \mathrm{H}, \mathrm{m}), 7.19-7.20(2 \mathrm{H}$, m), 7.27-7.30 (1H, d, $J=8.0 \mathrm{~Hz}), 7.40-7.44(2 \mathrm{H}, \mathrm{t}, J=7.8 \mathrm{~Hz}), 7.52-7.56(1 \mathrm{H}, \mathrm{t}, J=7.4$ $\mathrm{Hz}), 7.83-7.85(2 \mathrm{H}, \mathrm{d}, J=7.2 \mathrm{~Hz}) ;{ }^{13} \mathrm{C} \mathrm{NMR}\left(\mathrm{CDCl}_{3}, 101 \mathrm{MHz}\right) \delta 15.3,15.4,29.0,33.3$, $37.3,60.8,61.0,101.1,126.3,126.8,127.8,127.9,128.3,129.8,131.1,132.8,135.2$, 138.0, 138.3, 138.8, 196.7. HRMS (ESI) Calcd. for $\mathrm{C}_{23} \mathrm{H}_{26} \mathrm{NaO}_{3}\left(\mathrm{M}+\mathrm{Na}^{+}\right)$requires 373.1774, Found: 373.1775.

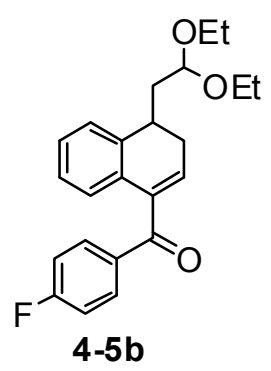

Data for 4-5b : a colorless oil; ${ }^{1} \mathrm{H}$ NMR $\left(\mathrm{CDCl}_{3}, 400 \mathrm{MHz}\right) \delta 1.07-1.11(3 \mathrm{H}, \mathrm{t}, J=7.2$ $\mathrm{Hz}), 1.17-1.21(3 \mathrm{H}, \mathrm{t}, J=7.2 \mathrm{~Hz}), 1.75-1.93(2 \mathrm{H}, \mathrm{m}), 2.38-2.44(1 \mathrm{H}, \mathrm{m}), 2.62-2.69(1 \mathrm{H}$, m), 2.96-3.01 (1H, m), 3.33-3.64 (4H, m), 4.38-4.41 (1H, t, $J=5.8 \mathrm{~Hz}), 6.31-6.34(1 \mathrm{H}$, dd, $J=6.4,3.2 \mathrm{~Hz}), 7.01-7.06(2 \mathrm{H}, \mathrm{t}, J=8.6 \mathrm{~Hz}), 7.08-7.12(1 \mathrm{H}, \mathrm{m}), 7.14-7.15(2 \mathrm{H}, \mathrm{m})$, 7.17-7.19 (1H, m), 7.81-7.84 (2H, m); ${ }^{13} \mathrm{C} \mathrm{NMR}\left(\mathrm{CDCl}_{3}, 101 \mathrm{MHz}\right) \delta$ 15.3, 15.4, 28.9, $33.3,37.3,60.9,61.0,101.1,115.5(\mathrm{~d}, J=22.7 \mathrm{~Hz}), 126.2,126.9,127.8,131.0,132.4$, $134.2,134.8,138.3,138.8,165.6(\mathrm{~d}, J=255.8 \mathrm{~Hz}), 195.2 ;{ }^{19} \mathrm{~F} \mathrm{NMR}\left(\mathrm{CDCl}_{3}, 376 \mathrm{MHz}\right) \delta$ $-105.43--105.40$ (m). HRMS (ESI) Calcd. for $\mathrm{C}_{23} \mathrm{H}_{25} \mathrm{FNaO}_{3}\left(\mathrm{M}+\mathrm{Na}^{+}\right)$requires 391.1680, Found: 391.1678. 


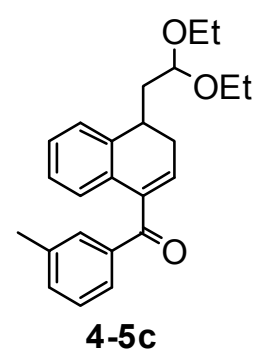

Data for 4-5c : colorless oil; ${ }^{1} \mathrm{H}$ NMR $\left(\mathrm{CDCl}_{3}, 400 \mathrm{MHz}\right) \delta 1.07-1.11(3 \mathrm{H}, \mathrm{t}, J=7.2 \mathrm{~Hz})$, 1.18-1.21 (3H, t, $J=7.0 \mathrm{~Hz}), 1.75-1.82(1 \mathrm{H}, \mathrm{m}), 1.88-1.95(1 \mathrm{H}, \mathrm{m}), 2.33(3 \mathrm{H}, \mathrm{s}), 2.36-$ $2.42(1 \mathrm{H}, \mathrm{m}), 2.63-2.69(1 \mathrm{H}, \mathrm{m}), 2.96-3.00(1 \mathrm{H}, \mathrm{m}), 3.34-3.55(3 \mathrm{H}, \mathrm{m}), 3.58-3.66(1 \mathrm{H}$, m), 4.38-4.41 (1H, t, $J=5.8 \mathrm{~Hz}), 6.32-6.34(1 \mathrm{H}, \mathrm{dd}, J=6.0,2.8 \mathrm{~Hz}), 7.08-7.15(3 \mathrm{H}, \mathrm{m})$, 7.21-7.26 (2H, m), 7.29-7.31 (1H, d, $J=7.6 \mathrm{~Hz}), 7.54-7.56(1 \mathrm{H}, \mathrm{d}, J=7.2 \mathrm{~Hz}), 7.63(1 \mathrm{H}$, s); ${ }^{13} \mathrm{C} \mathrm{NMR}\left(\mathrm{CDCl}_{3}, 101 \mathrm{MHz}\right) \delta 15.3,15.4,21.3,29.0,33.3,37.3,60.8,60.9,101.1$, $126.3,126.9,127.2,127.8,127.9,128.2,130.2,131.2,133.6,134.9,138.1,138.2,138.5$, 138.8, 197.0. HRMS (ESI) Calcd. for $\mathrm{C}_{24} \mathrm{H}_{28} \mathrm{NaO}_{3}\left(\mathrm{M}+\mathrm{Na}^{+}\right)$requires 387.1931, Found: 387.1927.

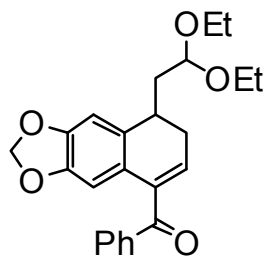

4-5d

Data for 4-5d : colorless oil; ${ }^{1} \mathrm{H}$ NMR $\left(\mathrm{CDCl}_{3}, 400 \mathrm{MHz}\right) \delta 1.08-1.12(3 \mathrm{H}, \mathrm{t}, J=7.2 \mathrm{~Hz})$, 1.17-1.20 (3H, t, $J=6.8 \mathrm{~Hz}), 1.72-1.87(2 \mathrm{H}, \mathrm{m}), 2.35-2.40(1 \mathrm{H}, \mathrm{m}), 2.57-2.65(1 \mathrm{H}, \mathrm{m})$, 2.85-2.91 (1H, m), 3.34-3.65 (4H, m), 4.37-4.40 (1H, t, J=6.0 Hz), $5.84(2 \mathrm{H}, \mathrm{s}), 6.25-$ $6.27(1 \mathrm{H}, \mathrm{dd}, J=6.0,3.0 \mathrm{~Hz}), 6.65(1 \mathrm{H}, \mathrm{s}), 6.82(1 \mathrm{H}, \mathrm{s}), 7.35-7.39(2 \mathrm{H}, \mathrm{t}, J=7.4 \mathrm{~Hz})$, 7.47-7.50 (1H, t, $J=7.4 \mathrm{~Hz}), 7.76-7.78(2 \mathrm{H}, \mathrm{d}, J=8 \mathrm{~Hz}) ;{ }^{13} \mathrm{C} \mathrm{NMR}\left(\mathrm{CDCl}_{3}, 101 \mathrm{MHz}\right) \delta$ $15.36,15.4,29.0,33.6,37.2,60.7,61.1,101.0,101.1,107.1,108.5,124.8,128.3,129.8$ 
132.7, 133.4, 134.0, 137.9, 138.1, 146.2, 146.8, 196.7. HRMS (ESI) Calcd. for $\mathrm{C}_{24} \mathrm{H}_{26} \mathrm{NaO}_{5}\left(\mathrm{M}+\mathrm{Na}^{+}\right)$requires 417.1672, Found: 417.1671.

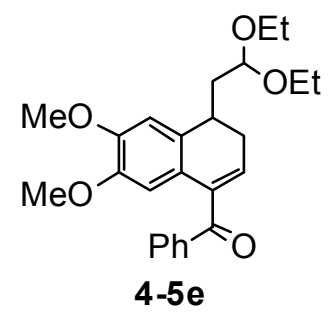

Data for 4-5e : colorless oil; ${ }^{1} \mathrm{H}$ NMR $\left(\mathrm{CDCl}_{3}, 400 \mathrm{MHz}\right) \delta 1.09-1.12(3 \mathrm{H}, \mathrm{t}, J=7.2 \mathrm{~Hz})$, $1.18-1.21(3 \mathrm{H}, \mathrm{t}, J=7.2 \mathrm{~Hz}), 1.72-1.89(2 \mathrm{H}, \mathrm{m}), 2.34-2.42(1 \mathrm{H}, \mathrm{m}), 2.59-2.67(1 \mathrm{H}, \mathrm{m})$, 2.88-2.94 (1H, m), 3.37-3.56 (3H, m), 3.60-3.65 (1H, m), $3.68(3 \mathrm{H}, \mathrm{s}), 3.83(3 \mathrm{H}, \mathrm{s}), 4.37-$ $4.40(1 \mathrm{H}, \mathrm{t}, J=6.0 \mathrm{~Hz}), 6.30-6.32(1 \mathrm{H}, \mathrm{dd}, J=6.0,3.0 \mathrm{~Hz}), 6.68(1 \mathrm{H}, \mathrm{s}), 6.95(1 \mathrm{H}, \mathrm{s})$, 7.36-7.40 (2H, t, $J=7.6 \mathrm{~Hz}), 7.47-7.51(1 \mathrm{H}, \mathrm{m}), 7.76-7.78(2 \mathrm{H}, \mathrm{d}, J=8 \mathrm{~Hz}) ;{ }^{13} \mathrm{C} \mathrm{NMR}$ $\left(\mathrm{CDCl}_{3}, 101 \mathrm{MHz}\right) \delta 15.38,15.44,29.1,33.2,37.4,55.8,55.9,61.0,61.2,101.3,109.9$, $111.1,123.6,128.3,129.8,131.8,132.7,134.9,137.5,147.3,148.4,196.9$. HRMS (ESI) Calcd. for $\mathrm{C}_{25} \mathrm{H}_{30} \mathrm{NaO}_{5}\left(\mathrm{M}+\mathrm{Na}^{+}\right)$requires 433.1985, Found: 433.1991 .

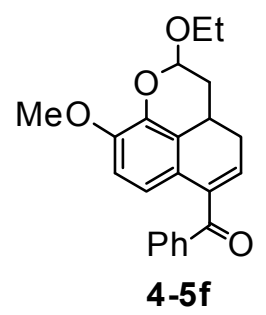

Data for 4-5f : semi solid; ${ }^{1} \mathrm{H}$ NMR $\left(\mathrm{CDCl}_{3}, 400 \mathrm{MHz}\right) \delta 1.23-1.27(3 \mathrm{H}, \mathrm{t}, J=7.2 \mathrm{~Hz})$, 1.76-1.84 (1H, q, $J=10.8 \mathrm{~Hz}), 2.10-2.25(2 \mathrm{H}, \mathrm{m}), 2.39-2.47(1 \mathrm{H}, \mathrm{m}), 3.07-3.14(1 \mathrm{H}, \mathrm{m})$, 3.64-3.68 (1H, m), $3.77(3 \mathrm{H}, \mathrm{s}), 4.08-4.13(1 \mathrm{H}, \mathrm{m}), 5.15-5.16(1 \mathrm{H}, \mathrm{d}, J=7.6 \mathrm{~Hz}), 6.27-$ $6.29(1 \mathrm{H}, \mathrm{dd}, J=7.0,2.8 \mathrm{~Hz}), 6.60-6.62(1 \mathrm{H}, \mathrm{d}, J=8.4 \mathrm{~Hz}), 6.74-6.76(1 \mathrm{H}, \mathrm{d}, J=8.4$ $\mathrm{Hz}), 7.35-7.38(2 \mathrm{H}, \mathrm{t}, J=7.6 \mathrm{~Hz}), 7.47-7.50(1 \mathrm{H}, \mathrm{t}, J=7.6 \mathrm{~Hz}), 7.78-7.80(2 \mathrm{H}, \mathrm{d}, J=7.2$ $\mathrm{Hz}) ;{ }^{13} \mathrm{C} \mathrm{NMR}\left(\mathrm{CDCl}_{3}, 101 \mathrm{MHz}\right) \delta 15.2,29.6,29.9,34.2,55.9,64.8,100.0,109.9,118.4$, 
120.7, 124.4, 128.3, 129.9, 132.8, 133.1, 137.9, 138.8, 141.5, 148.3, 196.9. HRMS (ESI)

Calcd. for $\mathrm{C}_{22} \mathrm{H}_{22} \mathrm{NaO}_{4}\left(\mathrm{M}+\mathrm{Na}^{+}\right)$requires 373.1410, Found: 373.1414 .

General procedure for the formation of isochromenes (4-6):

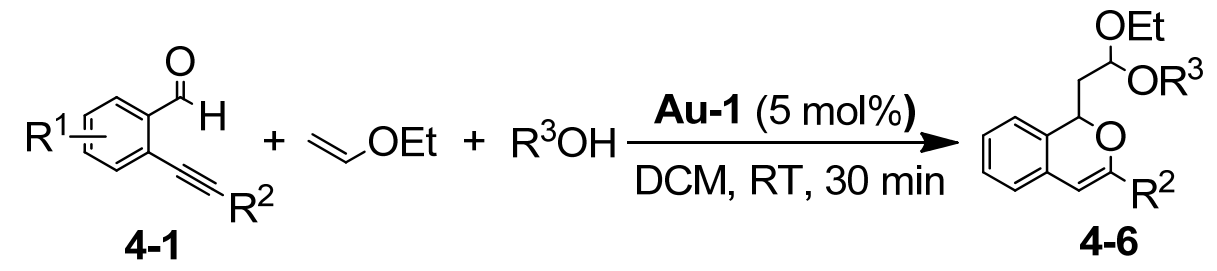

Au-1 (0.0072 mmol) was added to a solution of 2-alkynylbenzaldehyde $1(0.145 \mathrm{mmol}$, ethyl vinyl ether $(0.72 \mathrm{mmol})$ and ethanol $(0.28 \mathrm{mmol})$ in DCM $(1.0 \mathrm{~mL})$. The mixture was stirred for $30 \mathrm{~min}$ at room temperature. The reaction mixture was quenched with water and extracted with DCM. The solvent in the organic layer was removed under reduced pressure and the residue was subjected to flash column chromatography (eluent: ethyl acetate/n-hexane $=1 / 10$ ) to give product 4-6 as a colorless oil.

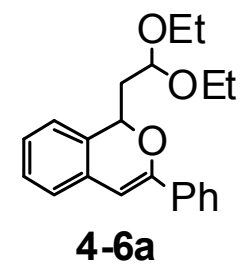

Data for 4-6a : colorless oil; ${ }^{1} \mathrm{H}$ NMR $\left(\mathrm{CDCl}_{3}, 400 \mathrm{MHz}\right) \delta 1.21-1.24(3 \mathrm{H}, \mathrm{t}, J=7.6 \mathrm{~Hz})$, 1.27-1.31 (3H, t, $J=7.2 \mathrm{~Hz}), 2.09-2.16(1 \mathrm{H}, \mathrm{m}), 2.42-2.49(1 \mathrm{H}, \mathrm{m}), 3.51-3.59(1 \mathrm{H}, \mathrm{m})$, 3.61-3.71 (2H, m), 3.76-3.84 (1H, m), 4.90-4.93 (1H, dd, $J=7.6,3.8 \mathrm{~Hz}), 5.50-5.53(1 \mathrm{H}$, dd, $J=9.6,4.0 \mathrm{~Hz}), 6.47(1 \mathrm{H}, \mathrm{s}), 7.09-7.13(2 \mathrm{H}, \mathrm{t}, J=8.0 \mathrm{~Hz}), 7.18-7.27(2 \mathrm{H}, \mathrm{m}), 7.34-$ $7.43(3 \mathrm{H}, \mathrm{m}), 7.76-7.78(2 \mathrm{H}, \mathrm{m}) ;{ }^{13} \mathrm{C} \mathrm{NMR}\left(\mathrm{CDCl}_{3}, 101 \mathrm{MHz}\right) \delta$ 15.39, 15.41, 37.9, 60.9, $62.2,75.0,99.9,100.6,123.7,124.0,124.9,126.6,128.0,128.4,128.7,130.9,131.0$ 
134.6, 151.3. HRMS (ESI) Calcd. for $\mathrm{C}_{21} \mathrm{H}_{24} \mathrm{NaO}_{3}\left(\mathrm{M}+\mathrm{Na}^{+}\right)$requires 347.1618, Found: 347.1622.

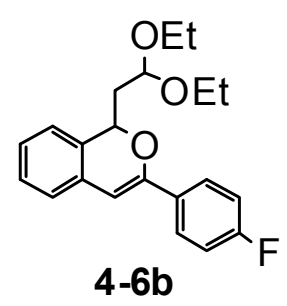

Data for 4-6b : colorless oil; ${ }^{1} \mathrm{H}$ NMR $\left(\mathrm{CDCl}_{3}, 400 \mathrm{MHz}\right) \delta 1.10-1.13(3 \mathrm{H}, \mathrm{t}, J=7.0 \mathrm{~Hz})$, $1.15-1.19(3 \mathrm{H}, \mathrm{t}, J=7.0 \mathrm{~Hz}), 1.97-2.04(1 \mathrm{H}, \mathrm{m}), 2.29-2.35(1 \mathrm{H}, \mathrm{m}), 3.40-3.60(3 \mathrm{H}, \mathrm{m})$, 3.63-3.71 (1H, m), 4.75-4.78 (1H, dd, $J=7.6,3.8 \mathrm{~Hz}), 5.36-5.40(1 \mathrm{H}, \mathrm{dd}, J=9.4,4.2$ $\mathrm{Hz}), 6.28(1 \mathrm{H}, \mathrm{s}), 6.96-7.01(4 \mathrm{H}, \mathrm{m})$, 7.07-7.11 (1H, m), 7.13-7.17 (1H, m), 7.61-7.64 $(2 \mathrm{H}, \mathrm{m}) ;{ }^{13} \mathrm{C} \mathrm{NMR}\left(\mathrm{CDCl}_{3}, 101 \mathrm{MHz}\right) \delta$ 15.3, 15.4, 37.8, 60.8, 62.1, 75.0, 99.8, 100.2, $115.3(\mathrm{~d}, J=21.7 \mathrm{~Hz}), 123.7,123.9,126.7,126.8,128.1,130.7,150.4,163.1(\mathrm{~d}, J=$ $247.1 \mathrm{~Hz}) ;{ }^{19} \mathrm{~F} \mathrm{NMR}\left(\mathrm{CDCl}_{3}, 376 \mathrm{MHz}\right) \delta-112.50$ - -112.43 (m). HRMS (ESI) Calcd. for $\mathrm{C}_{21} \mathrm{H}_{23} \mathrm{FNaO}_{3}\left(\mathrm{M}+\mathrm{Na}^{+}\right)$requires 365.1523, Found: 365.1523 .

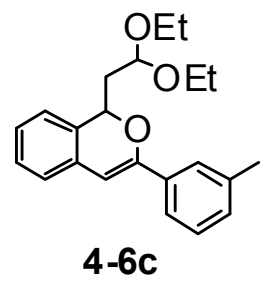

Data for 4-6c : colorless oil; ${ }^{1} \mathrm{H}$ NMR $\left(\mathrm{CDCl}_{3}, 400 \mathrm{MHz}\right) \delta 1.10-1.14(3 \mathrm{H}, \mathrm{t}, J=6.8 \mathrm{~Hz})$, 1.16-1.20 (3H, t, $J=7.2 \mathrm{~Hz}), 1.97-2.04(1 \mathrm{H}, \mathrm{m}), 2.31(3 \mathrm{H}, \mathrm{s}), 2.33-2.37(1 \mathrm{H}, \mathrm{m}), 3.40-$ $3.48(1 \mathrm{H}, \mathrm{m}), 3.52-3.60(2 \mathrm{H}, \mathrm{m}), 3.65-3.73(1 \mathrm{H}, \mathrm{m}), 4.78-4.81(1 \mathrm{H}, \mathrm{dd}, J=7.6,3.8 \mathrm{~Hz})$, 5.37-5.40 (1H, dd, $J=9.2,4.0 \mathrm{~Hz}), 6.35(1 \mathrm{H}, \mathrm{s}), 6.98-7.02(2 \mathrm{H}, \mathrm{t}, J=8.0 \mathrm{~Hz}), 7.07-7.10$ $(2 \mathrm{H}, \mathrm{t}, J=7.6 \mathrm{~Hz}), 7.13-7.21(2 \mathrm{H}, \mathrm{m}), 7.45-7.47(2 \mathrm{H}, \mathrm{d}, J=8.0 \mathrm{~Hz}) ;{ }^{13} \mathrm{C} \mathrm{NMR}\left(\mathrm{CDCl}_{3}\right.$, $101 \mathrm{MHz}) \delta 15.36,15.38,21.5,37.8,60.8,62.2,74.9,99.8,100.5,122.1,123.7,123.9$, 
125.5, 126.5, 128.0, 128.3, 129.5, 130.9, 134.5, 137.9, 151.4. HRMS (ESI) Calcd. for $\mathrm{C}_{22} \mathrm{H}_{26} \mathrm{NaO}_{3}\left(\mathrm{M}+\mathrm{Na}^{+}\right)$requires 361.1774, Found: 361.1777 .

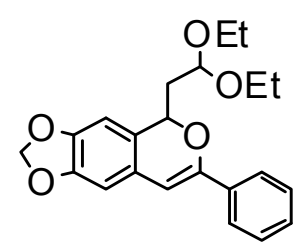

4-6d

Data for 4-6d : colorless oil; ${ }^{1} \mathrm{H}$ NMR $\left(\mathrm{CDCl}_{3}, 400 \mathrm{MHz}\right) \delta 1.10-1.13(3 \mathrm{H}, \mathrm{t}, J=7.2 \mathrm{~Hz})$, 1.16-1.20 (3H, t, $J=7.2 \mathrm{~Hz}), 1.90-1.97(1 \mathrm{H}, \mathrm{m}), 2.26-2.33(1 \mathrm{H}, \mathrm{m}), 3.39-3.47(1 \mathrm{H}, \mathrm{m})$, 3.50-3.59 (2H, m), 3.65-3.72 (1H, m), 4.75-4.78 (1H, dd, $J=7.8,4.0 \mathrm{~Hz}), 5.28-5.31(1 \mathrm{H}$, dd, $J=9.2,4.0 \mathrm{~Hz}), 5.86(2 \mathrm{H}, \mathrm{s}), 6.27(1 \mathrm{H}, \mathrm{s}), 6.53-6.54(2 \mathrm{H}, \mathrm{d}, J=4.8 \mathrm{~Hz}), 7.22-7.26$

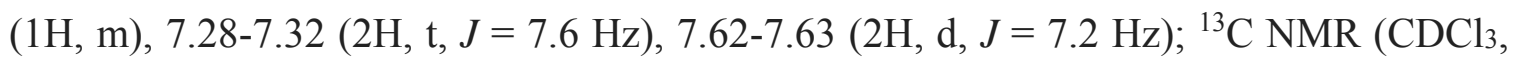
$101 \mathrm{MHz}) \delta 15.3,15.4,37.8,60.8,62.3,74.8,99.8,100.7,100.9,104.8,104.9,124.6$, 125.2, 128.3, 128.5, 134.5, 146.3, 147.2, 149.7. HRMS (ESI) Calcd. For $\mathrm{C}_{22} \mathrm{H}_{24} \mathrm{NaO}_{5}$ $\left(\mathrm{M}+\mathrm{Na}^{+}\right)$requires 391.1516, Found: 391.1519.

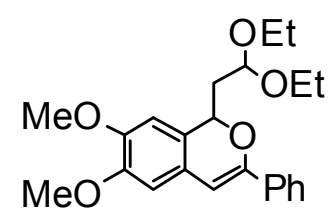

4-6e

Data for 4-6e : colorless oil; ${ }^{1} \mathrm{H}$ NMR $\left(\mathrm{CDCl}_{3}, 400 \mathrm{MHz}\right) \delta 1.10-1.14(3 \mathrm{H}, \mathrm{t}, J=7.2 \mathrm{~Hz})$, 1.18-1.22 (3H, t, $J=7.2 \mathrm{~Hz}), 1.93-1.99(1 \mathrm{H}, \mathrm{m}), 2.28-2.35(1 \mathrm{H}, \mathrm{m}), 3.40-3.47(1 \mathrm{H}, \mathrm{m})$, 3.55-3.60 (2H, m), 3.70-3.74 (1H, m), $3.81(3 \mathrm{H}, \mathrm{s}), 3.83(3 \mathrm{H}, \mathrm{m}), 4.78-4.81(1 \mathrm{H}, \mathrm{dd}, J=$ 8, $3.8 \mathrm{~Hz}), 5.34-5.37(1 \mathrm{H}, \mathrm{dd}, J=10.0,4.0 \mathrm{~Hz}), 6.31(1 \mathrm{H}, \mathrm{s}), 6.56-6.59(2 \mathrm{H}, \mathrm{d}, J=13.6$ $\mathrm{Hz}), 7.24-7.28(1 \mathrm{H}, \mathrm{t}, J=7.2 \mathrm{~Hz}), 7.30-7.32(2 \mathrm{H}, \mathrm{t}, J=6.8 \mathrm{~Hz}), 7.63-7.65(2 \mathrm{H}, \mathrm{d}, J=7.6$ $\mathrm{Hz}) ;{ }^{13} \mathrm{C} \mathrm{NMR}\left(\mathrm{CDCl}_{3}, 101 \mathrm{MHz}\right) \delta 15.36,15.39,38.1,56.0,56.2,60.9,62.6,74.7,100.0$, 
$100.3,107.6,123.3,123.8,124.5,128.3,128.4,134.7,148.0,148.7,149.6$. HRMS (ESI)

Calcd. for $\mathrm{C}_{23} \mathrm{H}_{28} \mathrm{NaO}_{5}\left(\mathrm{M}+\mathrm{Na}^{+}\right)$requires 407.1829, Found: 407.1828.

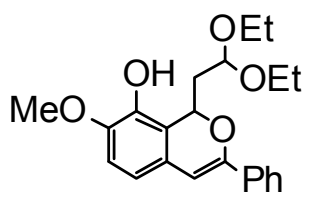

\section{4-6f}

Data for 4-6f : colorless oil; ${ }^{1} \mathrm{H} \mathrm{NMR}\left(\mathrm{CDCl}_{3}, 500 \mathrm{MHz}\right) \delta 1.13-1.16(3 \mathrm{H}, \mathrm{t}, J=7.0 \mathrm{~Hz})$, 1.27-1.30 (3H, t, $J=7.0 \mathrm{~Hz}), 1.94-1.99(1 \mathrm{H}, \mathrm{m}), 2.32-2.38(1 \mathrm{H}, \mathrm{m}), 3.47-3.51(1 \mathrm{H}, \mathrm{m})$, 3.60-3.67 (2H, m), 3.76-3.80 (1H, m), $3.89(3 \mathrm{H}, \mathrm{s}), 4.89-4.91(1 \mathrm{H}, \mathrm{dd}, J=7.8,3.8 \mathrm{~Hz})$, $5.80(1 \mathrm{H}, \mathrm{s}), \quad 5.89-5.92(1 \mathrm{H}, \mathrm{dd}, J=9.5,4.0 \mathrm{~Hz}), 6.37(1 \mathrm{H}, \mathrm{s}), 6.62-6.64(1 \mathrm{H}, \mathrm{d}, J=8.0$ $\mathrm{Hz}), 6.74-6.76(1 \mathrm{H}, \mathrm{d}, J=8.5 \mathrm{~Hz}), 7.29-7.32(1 \mathrm{H}, \mathrm{t}, J=7.5 \mathrm{~Hz}), 7.36-7.39(2 \mathrm{H}, \mathrm{t}, J=7.5$ $\mathrm{Hz}), 7.72-7.74(2 \mathrm{H}, \mathrm{d}, J=8.0 \mathrm{~Hz}) ;{ }^{13} \mathrm{C} \mathrm{NMR}\left(\mathrm{CDCl}_{3}, 126 \mathrm{MHz}\right) \delta 15.2,15.4,36.9,56.1$, $60.76,60.82,70.1,99.3,99.8,109.8,115.6,117.6,124.2,124.5,128.3,135.0,140.3$ 145.9, 148.6. HRMS (ESI) Calcd. for $\mathrm{C}_{22} \mathrm{H}_{26} \mathrm{NaO}_{5}\left(\mathrm{M}+\mathrm{Na}^{+}\right)$requires 393.1672, Found: 393.1683.

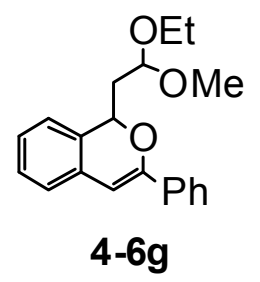

Data for 4-6g : colorless oil; ${ }^{1} \mathrm{H}$ NMR $\left(\mathrm{CDCl}_{3}, 400 \mathrm{MHz}\right) \delta 1.20-1.23(3 \mathrm{H}, \mathrm{t}, J=7.0 \mathrm{~Hz})$, 2.07-2.14 (1H, m), 2.40-2.47 (1H, m), $3.45(3 \mathrm{H}, \mathrm{s}), 3.50-3.56(1 \mathrm{H}, \mathrm{m}), 3.62-3.69(1 \mathrm{H}, \mathrm{m})$, 4.83-4.86 (1H, dd, $J=8.0,4.0 \mathrm{~Hz}), 5.47-5.50(1 \mathrm{H}, \mathrm{dd}, J=10.0,4.2 \mathrm{~Hz}), 6.46(1 \mathrm{H}, \mathrm{s})$, 7.06-7.12 (2H, m), 7.16-7.26 (2H, m), 7.33-7.42 (3H, m), 7.75-7.77 (2H, d, $J=8.0 \mathrm{~Hz})$; ${ }^{13} \mathrm{C} \mathrm{NMR}\left(\mathrm{CDCl}_{3}, 101 \mathrm{MHz}\right) \delta 15.3,37.4,53.5,61.1,74.9,100.6,100.7,123.7,124.0$, 
124.9, 126.7, 128.1, 128.4, 128.8, 130.88, 130.91, 134.6, 151.3. HRMS (ESI) Calcd. for $\mathrm{C}_{20} \mathrm{H}_{22} \mathrm{NaO}_{3}\left(\mathrm{M}+\mathrm{Na}^{+}\right)$requires 333.1461, Found: 333.1470 .

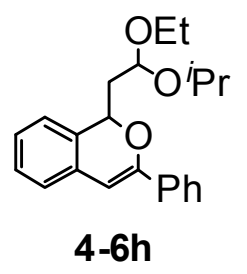

Data for 4-6h : colorless oil; ${ }^{1} \mathrm{H}$ NMR $\left(\mathrm{CDCl}_{3}, 400 \mathrm{MHz}\right) \delta 1.17-1.21(3 \mathrm{H}, \mathrm{t}, J=7.0 \mathrm{~Hz})$, 1.24-1.29 (6H, m), 2.00-2.07 (1H, m), 2.41-2.49 (1H, m), 3.50-3.63 (2H, m), 3.96-4.02 (1H, m), 4.96-4.99 (1H, dd, $J=8.0,3.2 \mathrm{~Hz}), 5.47-5.51(1 \mathrm{H}, \mathrm{dd}, J=10.4,3.8 \mathrm{~Hz}), 6.46$ $(1 \mathrm{H}, \mathrm{s}), 7.09-7.11(2 \mathrm{H}, \mathrm{m}), 7.16-7.26(2 \mathrm{H}, \mathrm{m}), 7.32-7.41(3 \mathrm{H}, \mathrm{m}), 7.74-7.76(2 \mathrm{H}, \mathrm{d}, J=$ $7.2 \mathrm{~Hz}) ;{ }^{13} \mathrm{C} \mathrm{NMR}\left(\mathrm{CDCl}_{3}, 101 \mathrm{MHz}\right) \delta 15.4,22.3,23.4,38.5,59.6,69.4,75.0,98.2$, $100.5,123.7,124.0,124.8,126.6,128.0,128.3,128.7,130.8,131.0,134.6,151.1$. HRMS (ESI) Calcd. for $\mathrm{C}_{22} \mathrm{H}_{26} \mathrm{NaO}_{3}\left(\mathrm{M}+\mathrm{Na}^{+}\right)$requires 361.1774, Found: 361.1776 .

\section{General procedure for the formation of bicyclo[2.2.2]octanes (4-7):}
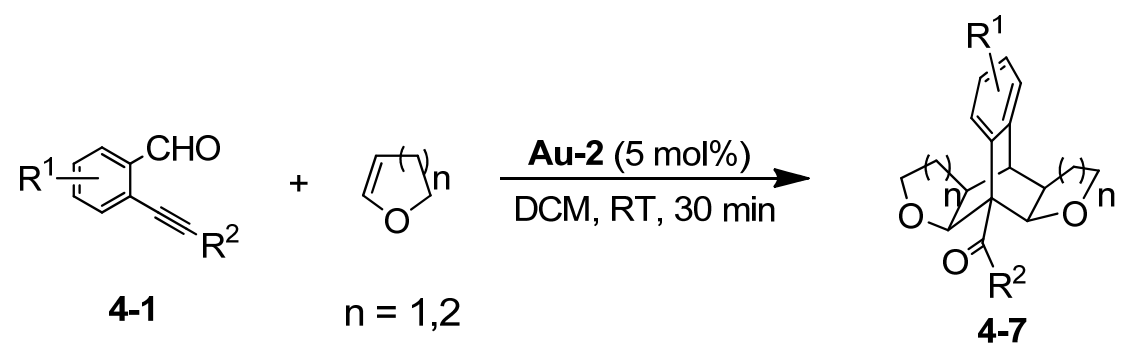

Au-2 (0.0072 mmol) was added to a solution of 2-alkynylbenzaldehyde 4-1 $(0.145 \mathrm{mmol})$, 2,3-dihydrofuran $(0.72 \mathrm{mmol})$ in DCM $(1.0 \mathrm{~mL})$. The mixture was stirred for $30 \mathrm{~min}$ at room temperature. The reaction mixture was quenched with water and extracted with DCM. The solvent in the organic layer was removed under reduced pressure and the 
residue was subjected to a flash column chromatography (eluent: ethyl acetate/n-hexane $=1 / 10)$ to give product 4-7.

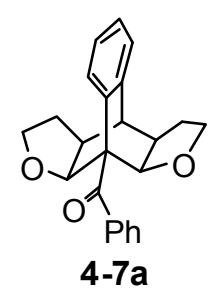

Data for 4-7a: white solid; mp. 188.2-193.0 ${ }^{\circ} \mathrm{C} ;{ }^{1} \mathrm{H}$ NMR $\left(\mathrm{CDCl}_{3}, 400 \mathrm{MHz}\right) \delta 1.13-1.21$ $(2 \mathrm{H}, \mathrm{m}), 1.81-1.89(2 \mathrm{H}, \mathrm{m}), 2.54-2.61(2 \mathrm{H}, \mathrm{m}), 2.91-2.97(3 \mathrm{H}, \mathrm{m}), 3.29-3.35(2 \mathrm{H}, \mathrm{q}, J=$ $7.2 \mathrm{~Hz}), 4.48-4.50(2 \mathrm{H}, \mathrm{d}, J=8.4 \mathrm{~Hz}), 7.05-7.18(3 \mathrm{H}, \mathrm{m}), 7.30-7.34(2 \mathrm{H}, \mathrm{t}, J=7.6 \mathrm{~Hz})$, 7.38-7.41 (1H, t, $J=6.8 \mathrm{~Hz}), 7.58-7.59(1 \mathrm{H}, \mathrm{d}, J=7.2 \mathrm{~Hz}), 7.83-7.85(2 \mathrm{H}, \mathrm{d}, J=8.4 \mathrm{~Hz})$; ${ }^{13} \mathrm{C}$ NMR $\left(\mathrm{CDCl}_{3}, 101 \mathrm{MHz}\right) \delta 30.3,43.8,44.2,62.2,68.5,81.0,126.4,126.8,127.3$, 127.6, 129.0, 129.6, 130.8, 134.9, 137.8, 140.3, 204.0. HRMS (ESI) Calcd. for $\mathrm{C}_{23} \mathrm{H}_{23} \mathrm{O}_{3}$ $\left(\mathrm{M}+\mathrm{H}^{+}\right)$requires 347.1642, Found: 347.1642.

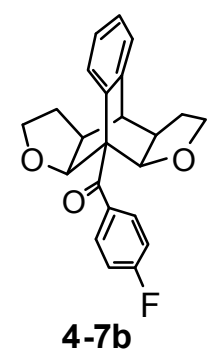

Data for 4-7b: white solid; mp. 185.0-188.0 ${ }^{\circ} \mathrm{C} ;{ }^{1} \mathrm{H}$ NMR $\left(\mathrm{CDCl}_{3}, 500 \mathrm{MHz}\right) \delta 1.14-1.21$ $(2 \mathrm{H}, \mathrm{m}), 1.84-1.90(2 \mathrm{H}, \mathrm{m}), 2.58-2.63(2 \mathrm{H}, \mathrm{m}), 2.94-3.0(3 \mathrm{H}, \mathrm{m}), 3.30-3.34(2 \mathrm{H}, \mathrm{q}, J=$ 7.6 Hz), 4.49-4.51 (2H, d, $J=8.5 \mathrm{~Hz}), 6.96-7.0(2 \mathrm{H}, \mathrm{t}, J=8.8 \mathrm{~Hz}), 7.06-7.09(2 \mathrm{H}, \mathrm{t}, J=$ 8.2 Hz), 7.15-7.19 (1H, m), 7.29-7.31 (1H, d, $J=7.5 \mathrm{~Hz}), 7.97-8.0(2 \mathrm{H}, \mathrm{m}) ;{ }^{13} \mathrm{C}$ NMR $\left(\mathrm{CDCl}_{3}, 101 \mathrm{MHz}\right) \delta 30.3,43.9,44.1,62.5,68.4,81.2,114.5(\mathrm{~d}, J=21.9 \mathrm{~Hz}), 126.5$, 126.6, 127.5, 129.7, 132.7, 135.0, 135.9, 137.8, 164.7 (d, $J=253.5 \mathrm{~Hz}), 201.5 ;{ }^{19} \mathrm{~F}$ NMR 
$\left(\mathrm{CDCl}_{3}, 470 \mathrm{MHz}\right) \delta-108.08--108.02$ (m), HRMS (ESI) Calcd. for $\mathrm{C}_{23} \mathrm{H}_{21} \mathrm{FNaO}_{3}$ $\left(\mathrm{M}+\mathrm{Na}^{+}\right)$requires 387.1367, Found: 387.1371.

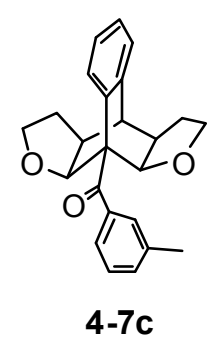

Data for 4-7c: white solid; mp. 179.2-184.8 ${ }^{\circ} \mathrm{C} ;{ }^{1} \mathrm{H}$ NMR $\left(\mathrm{CDCl}_{3}, 400 \mathrm{MHz}\right) \delta 1.15-1.21$ $(2 \mathrm{H}, \mathrm{m}), 1.82-1.91(2 \mathrm{H}, \mathrm{m}), 2.31(3 \mathrm{H}, \mathrm{s}), 2.56-2.61(2 \mathrm{H}, \mathrm{m}), 2.89-2.94(2 \mathrm{H}, \mathrm{m}), 2.96-$ $2.98(1 \mathrm{H}, \mathrm{m}), 3.30-3.35(2 \mathrm{H}, \mathrm{q}, J=7.6 \mathrm{~Hz}), 4.49-4.51(2 \mathrm{H}, \mathrm{d}, J=8.4 \mathrm{~Hz}), 7.05-7.07(1 \mathrm{H}$, m), 7.12-7.15 (2H, m), 7.18-7.21 (2H, m), $7.59(1 \mathrm{H}, \mathrm{s}), 7.64-7.66(2 \mathrm{H}, \mathrm{m}) ;{ }^{13} \mathrm{C}$ NMR $\left(\mathrm{CDCl}_{3}, 101 \mathrm{MHz}\right) \delta 21.4,30.3,43.8,44.2,62.0,68.5,80.9,125.8,126.3,126.8,127.2$, 127.4, 129.1, 129.5, 131.4, 134.9, 137.5, 137.7, 140.5, 204.4. HRMS (ESI) Calcd. for $\mathrm{C}_{24} \mathrm{H}_{24} \mathrm{NaO}_{3}\left(\mathrm{M}+\mathrm{Na}^{+}\right)$requires 383.1618, Found: 383.1617.

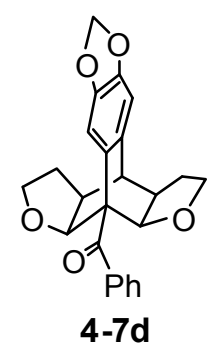

Data for 4-7d: semi solid; ${ }^{1} \mathrm{H}$ NMR $\left(\mathrm{CDCl}_{3}, 400 \mathrm{MHz}\right) \delta$ 1.18-1.26 (2H, m), 1.80-1.89 $(2 \mathrm{H}, \mathrm{m}), 2.48-2.54(2 \mathrm{H}, \mathrm{m}), 2.88-2.90(1 \mathrm{H}, \mathrm{m}), 3.02-3.08(2 \mathrm{H}, \mathrm{m}), 3.33-3.38(2 \mathrm{H}, \mathrm{q}, J=$ $7.8 \mathrm{~Hz}), 4.41-4.43(2 \mathrm{H}, \mathrm{d}, J=8.8 \mathrm{~Hz}), 5.84(2 \mathrm{H}, \mathrm{s}), 6.59(1 \mathrm{H}, \mathrm{s}), 7.31-7.35(3 \mathrm{H}, \mathrm{m})$, 7.38-7.42 (1H, m), 7.76-7.77 (2H, d, $J=7.2 \mathrm{~Hz}) ;{ }^{13} \mathrm{C} \mathrm{NMR}\left(\mathrm{CDCl}_{3}, 101 \mathrm{MHz}\right) \delta 30.3$, $43.8,44.3,62.0,68.6,80.7,100.6,107.9,110.9,127.6,127.8,128.3,128.4,130.4,130.5$, 
131.1, 140.4, 146.1, 146.5, 204.5. HRMS (ESI) Calcd. for $\mathrm{C}_{24} \mathrm{H}_{22} \mathrm{NaO}_{5}\left(\mathrm{M}+\mathrm{Na}^{+}\right)$requires 413.1359, Found: 413.1365.

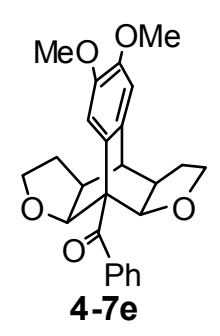

Data for 4-7e: yellow oil; ${ }^{1} \mathrm{H}$ NMR $\left(\mathrm{CDCl}_{3}, 400 \mathrm{MHz}\right) \delta$ 1.16-1.24 (2H, m), 1.81-1.91 (2H, m), 2.54-2.57 (2H, q, $J=7.8 \mathrm{~Hz}), 2.90-3.02(3 \mathrm{H}, \mathrm{m}), 3.32-3.38(2 \mathrm{H}, \mathrm{q}, J=7.8 \mathrm{~Hz})$, $3.54(3 \mathrm{H}, \mathrm{s}), 3.83(3 \mathrm{H}, \mathrm{s}), 4.48-4.50(2 \mathrm{H}, \mathrm{d}, J=8.4 \mathrm{~Hz}), 6.60(1 \mathrm{H}, \mathrm{s}), 7.17(1 \mathrm{H}, \mathrm{s}), 7.30-$ $7.35(2 \mathrm{H}, \mathrm{m}), 7.38-7.40(1 \mathrm{H}, \mathrm{m}), 7.84-7.86(2 \mathrm{H}, \mathrm{d}, J=7.2 \mathrm{~Hz}) ;{ }^{13} \mathrm{C} \mathrm{NMR}\left(\mathrm{CDCl}_{3}, 101\right.$ MHz) $\delta 30.3,43.9,55.4,55.9,61.9,68.6,81.2,110.4,113.5,126.6,127.7,128.9,129.9$, 130.7, 147.4, 204.7. HRMS (ESI) Calcd. for $\mathrm{C}_{25} \mathrm{H}_{26} \mathrm{NaO}_{5}\left(\mathrm{M}+\mathrm{Na}^{+}\right)$requires 429.1672, Found: 429.1678.

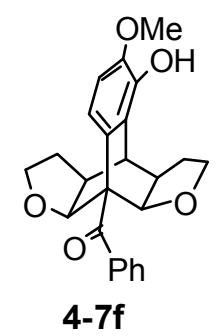

Data for 4-7f: yellow oil; ${ }^{1} \mathrm{H}$ NMR $\left(\mathrm{CDCl}_{3}, 400 \mathrm{MHz}\right) \delta 1.24-1.31(2 \mathrm{H}, \mathrm{m}), 1.92-1.99$ $(2 \mathrm{H}, \mathrm{m}), 2.62-2.68(2 \mathrm{H}, \mathrm{m}), 3.12-3.16(2 \mathrm{H}, \mathrm{m}), 3.40-3.45(2 \mathrm{H}, \mathrm{q}, J=7.8 \mathrm{~Hz}), 3.67-3.68$ $(1 \mathrm{H}, \mathrm{m}), 3.88(3 \mathrm{H}, \mathrm{s}), 4.52-4.54(2 \mathrm{H}, \mathrm{d}, J=8.5 \mathrm{~Hz}), 5.57(1 \mathrm{H}, \mathrm{s}), 6.69-6.70(1 \mathrm{H}, \mathrm{d}, J=$ $8.5 \mathrm{~Hz}), 7.19-7.20(1 \mathrm{H}, \mathrm{d}, J=8.5 \mathrm{~Hz}), 7.38-7.41(2 \mathrm{H}, \mathrm{t}, J=7.5 \mathrm{~Hz}), 7.46-7.49(1 \mathrm{H}, \mathrm{t}, J=$ $7.2 \mathrm{~Hz}), 7.90-7.92(2 \mathrm{H}, \mathrm{d}, J=7.5 \mathrm{~Hz}) ;{ }^{13} \mathrm{C} \mathrm{NMR}\left(\mathrm{CDCl}_{3}, 101 \mathrm{MHz}\right) \delta 30.3,36.0,43.4$, $55.7,61.7,68.6,80.9,108.3,121.1,127.6,128.8,130.6,140.5,142.5,144.7,204.3$. HRMS (ESI) Calcd. for $\mathrm{C}_{24} \mathrm{H}_{24} \mathrm{NaO}_{5}\left(\mathrm{M}+\mathrm{Na}^{+}\right)$requires 415.1516, Found: 415.1518 . 


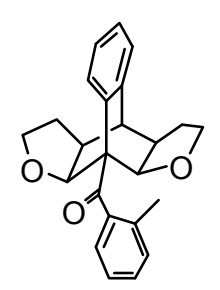

4-7g

Data for 4-7g: white solid; mp. 185.2-187.6 ${ }^{\circ} \mathrm{C} ;{ }^{1} \mathrm{H}$ NMR $\left(\mathrm{CDCl}_{3}, 400 \mathrm{MHz}\right) \delta 1.21-1.28$ (2H, m), 1.85-1.94 (2H, m), $2.43(3 \mathrm{H}, \mathrm{s}), 2.57-2.63(2 \mathrm{H}, \mathrm{m}), 2.94-3.01(3 \mathrm{H}, \mathrm{m}), 3.34-$ $3.40(2 \mathrm{H}, \mathrm{q}, J=7.6 \mathrm{~Hz}), 4.52-4.54(2 \mathrm{H}, \mathrm{d}, J=8.8 \mathrm{~Hz}), 7.11-7.13(1 \mathrm{H}, \mathrm{m}), 7.19-7.30(5 \mathrm{H}$, m), 7.76-7.78 $(1 \mathrm{H}, \mathrm{d}, J=7.6 \mathrm{~Hz}), 7.97-7.99(1 \mathrm{H}, \mathrm{t}, J=7.6 \mathrm{~Hz}) ;{ }^{13} \mathrm{C} \mathrm{NMR}\left(\mathrm{CDCl}_{3}, 101\right.$ MHz) $\delta 19.5,30.4,43.9,44.3,62.2,68.4,80.5,124.7,125.9,126.3,127.3,128.7,129.0$, 131.0, 134.7, 136.2, 137.6, 140.8, 207.1. HRMS (ESI) Calcd. for $\mathrm{C}_{24} \mathrm{H}_{24} \mathrm{NaO}_{3}\left(\mathrm{M}+\mathrm{Na}^{+}\right)$ requires 383.1618, Found: 383.1625 .

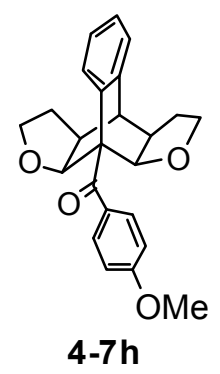

Data for 4-7h: white solid; mp. $149.8-152.5{ }^{\circ} \mathrm{C} ;{ }^{1} \mathrm{H}$ NMR $\left(\mathrm{CDCl}_{3}, 400 \mathrm{MHz}\right) \delta 1.13-1.21$ $(2 \mathrm{H}, \mathrm{m}), 1.82-1.91(2 \mathrm{H}, \mathrm{m}), 2.57-2.64(2 \mathrm{H}, \mathrm{m}), 2.93-3.0(3 \mathrm{H}, \mathrm{m}), 3.28-3.34(2 \mathrm{H}, \mathrm{q}, J=$ $7.6 \mathrm{~Hz}), 3.78(3 \mathrm{H}, \mathrm{s}), 4.52-4.54(2 \mathrm{H}, \mathrm{d}, J=8.8 \mathrm{~Hz}), 6.78-6.80(2 \mathrm{H}, \mathrm{d}, J=8.8 \mathrm{~Hz}), 7.02-$ $7.09(2 \mathrm{H}, \mathrm{m}), 7.13-7.18(1 \mathrm{H}, \mathrm{m}), 7.24-7.26(1 \mathrm{H}, \mathrm{d}, J=8.0 \mathrm{~Hz}), 8.00-8.02(2 \mathrm{H}, \mathrm{t}, J=8.8$ $\mathrm{Hz}) ;{ }^{13} \mathrm{C} \mathrm{NMR}\left(\mathrm{CDCl}_{3}, 101 \mathrm{MHz}\right) \delta 30.3,43.9,44.2,55.3,62.5,68.3,81.4,112.5,126.34$, 126.36, 127.4, 130.1, 132.2, 133.2, 135.6, 137.9, 162.4, 200.5. HRMS (ESI) Calcd. for $\mathrm{C}_{24} \mathrm{H}_{24} \mathrm{NaO}_{4}\left(\mathrm{M}+\mathrm{Na}^{+}\right)$requires 399.1567, Found: 399.1602. 


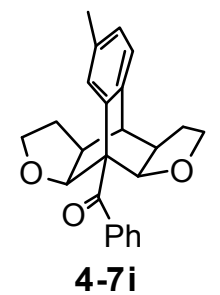

Data for 4-7i: semi solid; ${ }^{1} \mathrm{H}$ NMR $\left(\mathrm{CDCl}_{3}, 400 \mathrm{MHz}\right) \delta$ 1.14-1.24 (2H, m), 1.80-1.87 $(2 \mathrm{H}, \mathrm{m}), 2.18(3 \mathrm{H}, \mathrm{s}), 2.52-2.58(2 \mathrm{H}, \mathrm{m}), 2.92-3.0(3 \mathrm{H}, \mathrm{m}), 3.30-3.35(2 \mathrm{H}, \mathrm{q}, J=7.6 \mathrm{~Hz})$, 4.46-4.48 (2H, d, $J=9.2 \mathrm{~Hz}), 6.95(2 \mathrm{H}, \mathrm{s}), 7.31-7.35(2 \mathrm{H}, \mathrm{t}, J=7.6 \mathrm{~Hz}), 7.39-7.42(1 \mathrm{H}, \mathrm{t}$, $J=7.2 \mathrm{~Hz}), 7.49(1 \mathrm{H}, \mathrm{s}), 7.81-7.83(2 \mathrm{H}, \mathrm{d}, J=7.2 \mathrm{~Hz}) ;{ }^{13} \mathrm{C} \mathrm{NMR}\left(\mathrm{CDCl}_{3}, 101 \mathrm{MHz}\right) \delta$ $21.7,30.3,43.7,43.9,62.0,68.5,80.7,127.02,127.04,127.6,128.7,130.3,130.6,134.4$, 134.5, 136.3, 140.4, 204.3. HRMS (ESI) Calcd. for $\mathrm{C}_{24} \mathrm{H}_{24} \mathrm{NaO}_{3}\left(\mathrm{M}+\mathrm{Na}^{+}\right)$requires 383.1618, Found: 383.1619.

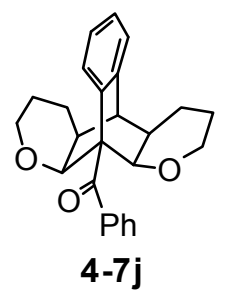

Data for 4-7j: white solid; mp. 188.0-194.0 ${ }^{\circ} \mathrm{C} ;{ }^{1} \mathrm{H}$ NMR $\left(\mathrm{CDCl}_{3}, 400 \mathrm{MHz}\right) \delta 0.66-0.77$ (2H, m), 1.23-1.29 (2H, m), 1.31-1.41 (2H, m), 1.53-1.63 (2H, m), 1.87-1.94 (2H, m), $2.54(1 \mathrm{H}, \mathrm{s}), 3.30-3.35(2 \mathrm{H}, \mathrm{m}), 3.62-3.69(2 \mathrm{H}, \mathrm{m}), 4.01-4.03(2 \mathrm{H}, \mathrm{d}, J=8.8 \mathrm{~Hz}), 7.02-$ $7.04(1 \mathrm{H}, \mathrm{d}, J=6.8 \mathrm{~Hz}), 7.13-7.23(2 \mathrm{H}, \mathrm{m}), 7.31-7.39(3 \mathrm{H}, \mathrm{m}), 7.44-7.45(2 \mathrm{H}, \mathrm{d}, J=6.4$ $\mathrm{Hz}), 8.18-8.20(1 \mathrm{H}, \mathrm{d}, J=7.2 \mathrm{~Hz}) ;{ }^{13} \mathrm{C} \mathrm{NMR}\left(\mathrm{CDCl}_{3}, 101 \mathrm{MHz}\right) \delta 20.4,21.6,40.6,45.7$, $60.5,63.0,75.5,125.9,126.56,126.65,126.7,127.9,128.6,129.4,133.9,139.0,141.5$ 206.3. HRMS (ESI) Calcd. for $\mathrm{C}_{25} \mathrm{H}_{26} \mathrm{NaO}_{3}\left(\mathrm{M}+\mathrm{Na}^{+}\right)$requires 397.1774, Found: 397.1779. 


\section{General procedure for the formation of dimer (4-9):}

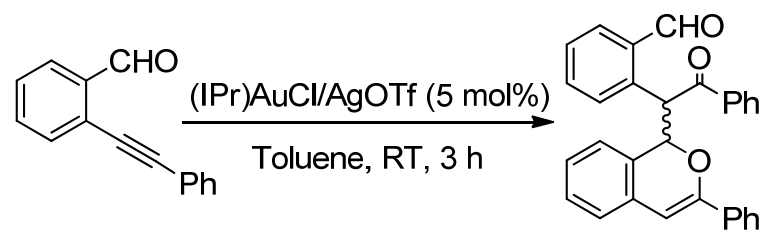

4-1a

4-9

(IPr)AuCl (0.0072 mmol) and AgOTf (10.0072 mmol) were added to a solution of 2alkynylbenzaldehyde 4-1a $(30 \mathrm{mg}, 0.145 \mathrm{mmol})$ in toluene $(1.0 \mathrm{~mL})$. The mixture was stirred for $3 \mathrm{~h}$ at room temperature. The reaction mixture was quenched with water and extracted with DCM. The solvent in the organic layer was removed under reduced pressure and the residue was subjected to flash column chromatography (eluent: ethyl acetate $/$ n-hexane $=1 / 10)$ to give product 4-9a (syn, $10.1 \mathrm{mg}, 32 \%)$ as yellow liquid and 4-9b (anti, $16.3 \mathrm{mg}, 52 \%$ ) as a white solid.

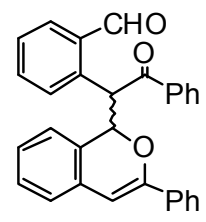

4-9a

Data for 4-9a (Syn diastereomer): yellow liquid; ${ }^{1} \mathrm{H}$ NMR $\left(\mathrm{CDCl}_{3}, 400 \mathrm{MHz}\right) \delta$ 6.28-6.31 $(1 \mathrm{H}, \mathrm{d}, J=9.6 \mathrm{~Hz}), 6.60(1 \mathrm{H}, \mathrm{s}), 6.99-7.08(4 \mathrm{H}, \mathrm{m}), 7.12-7.27(8 \mathrm{H}, \mathrm{m}), 7.36-7.50(3 \mathrm{H}$, m), 7.60-7.64 (1H, t, $J=7.4 \mathrm{~Hz}), 7.85-7.87(2 \mathrm{H}, \mathrm{d}, J=8.4 \mathrm{~Hz}), 7.99-8.01(1 \mathrm{H}, \mathrm{d}, J=7.6$ $\mathrm{Hz}), 9.64(1 \mathrm{H}, \mathrm{s}) ;{ }^{13} \mathrm{C} \mathrm{NMR}\left(\mathrm{CDCl}_{3}, 101 \mathrm{MHz}\right) \delta 49.1,79.8,101.0,124.4,124.5,126.0$, $126.5,127.6,127.9,128.36,128.4,128.6,128.7,129.6,129.8,130.4,133.2,133.5,133.8$, 134.7, 135.1, 136.9, 150.7, 192.9, 198.5. HRMS (ESI) Calcd. for $\mathrm{C}_{30} \mathrm{H}_{22} \mathrm{NaO}_{3}\left(\mathrm{M}+\mathrm{Na}^{+}\right)$ requires 453.1461 , Found: 453.1465 . 


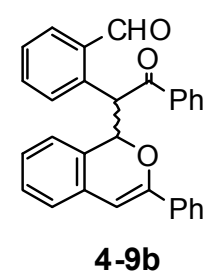

Data for 4-9b (Anti diastereomer): white solid; mp. 129.8-132.3 ${ }^{\circ} \mathrm{C}$; ${ }^{1} \mathrm{H}$ NMR $\left(\mathrm{CDCl}_{3}\right.$, $400 \mathrm{MHz}) \delta 5.92-5.94(1 \mathrm{H}, \mathrm{d}, J=7.6 \mathrm{~Hz}), 6.17-6.20(1 \mathrm{H}, \mathrm{d}, J=9.6 \mathrm{~Hz}), 6.58(1 \mathrm{H}, \mathrm{s})$, 6.65-6.68 (1H, t, $J=7.4 \mathrm{~Hz}), 7.03-7.11(3 \mathrm{H}, \mathrm{m}), 7.18-7.19(3 \mathrm{H}, \mathrm{m}), 7.24-7.28(2 \mathrm{H}, \mathrm{t}, J=$ 7.2 Hz), 7.35-7.39 (2H, t, $J=7.6 \mathrm{~Hz}), 7.46-7.55(4 \mathrm{H}, \mathrm{m}), 7.77-7.80(1 \mathrm{H}, \mathrm{d}, J=8.0 \mathrm{~Hz})$, 7.98-8.00 (2H, d, $J=8.0 \mathrm{~Hz}), 9.57(1 \mathrm{H}, \mathrm{s}) ;{ }^{13} \mathrm{C} \mathrm{NMR}\left(\mathrm{CDCl}_{3}, 101 \mathrm{MHz}\right) \delta 46.1,79.9$, $100.5,123.8,125.1,125.2,125.4,127.0,128.0,128.1,128.2,128.57,128.66,128.68$, 129.3, 131.3, 133.2, 133.6, 134.0, 134.5, 135.5, 136.9, 150.7, 191.9, 198.2. HRMS (ESI) Calcd. for $\mathrm{C}_{30} \mathrm{H}_{22} \mathrm{NaO}_{3}\left(\mathrm{M}+\mathrm{Na}^{+}\right)$requires 453.1461, Found: 453.1464 . 


\section{${ }^{1} \mathrm{H},{ }^{13} \mathrm{C}$ and ${ }^{19} \mathrm{~F}$ spectra of compound 4-5a,4-6a, 4-7a and 4-9.}

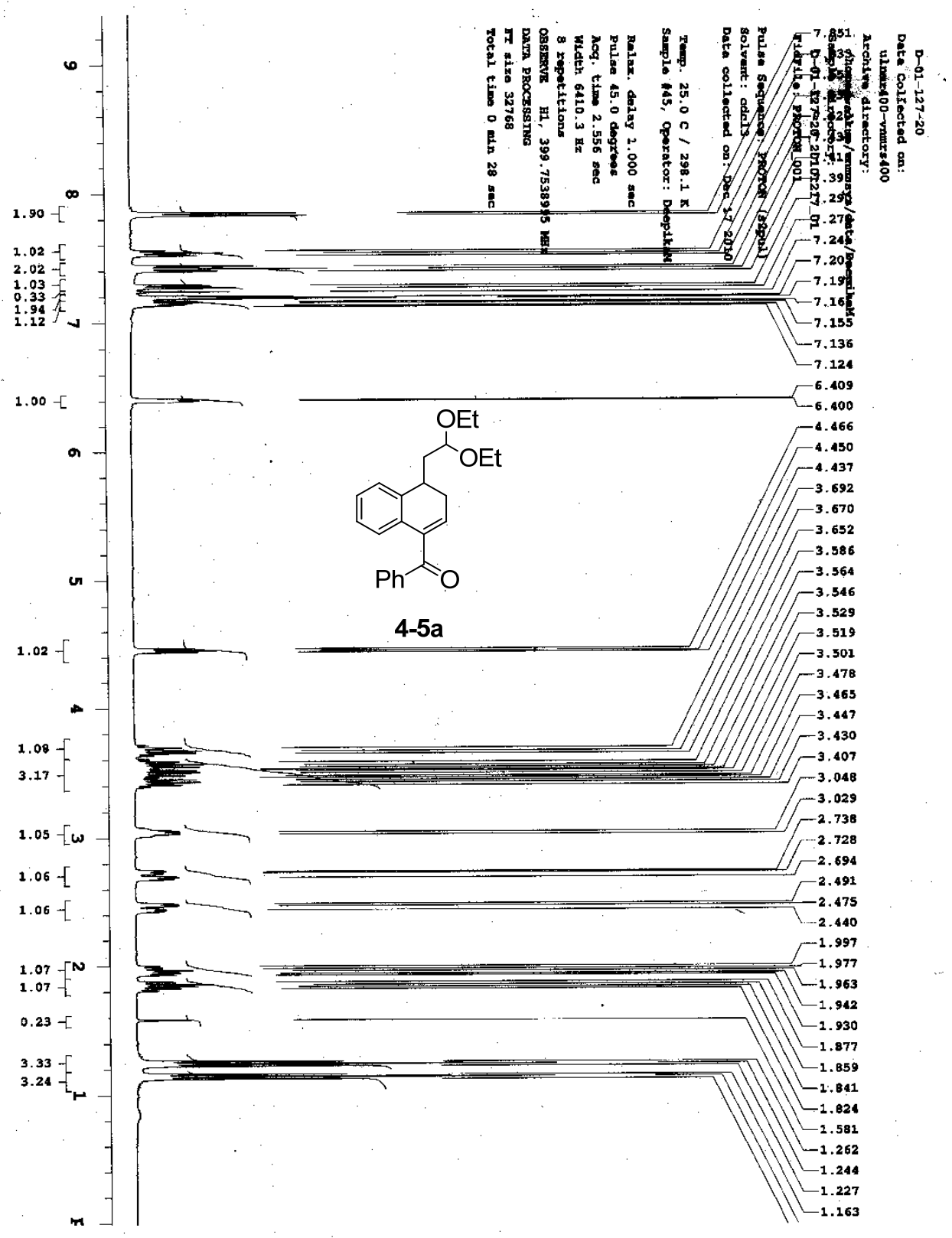




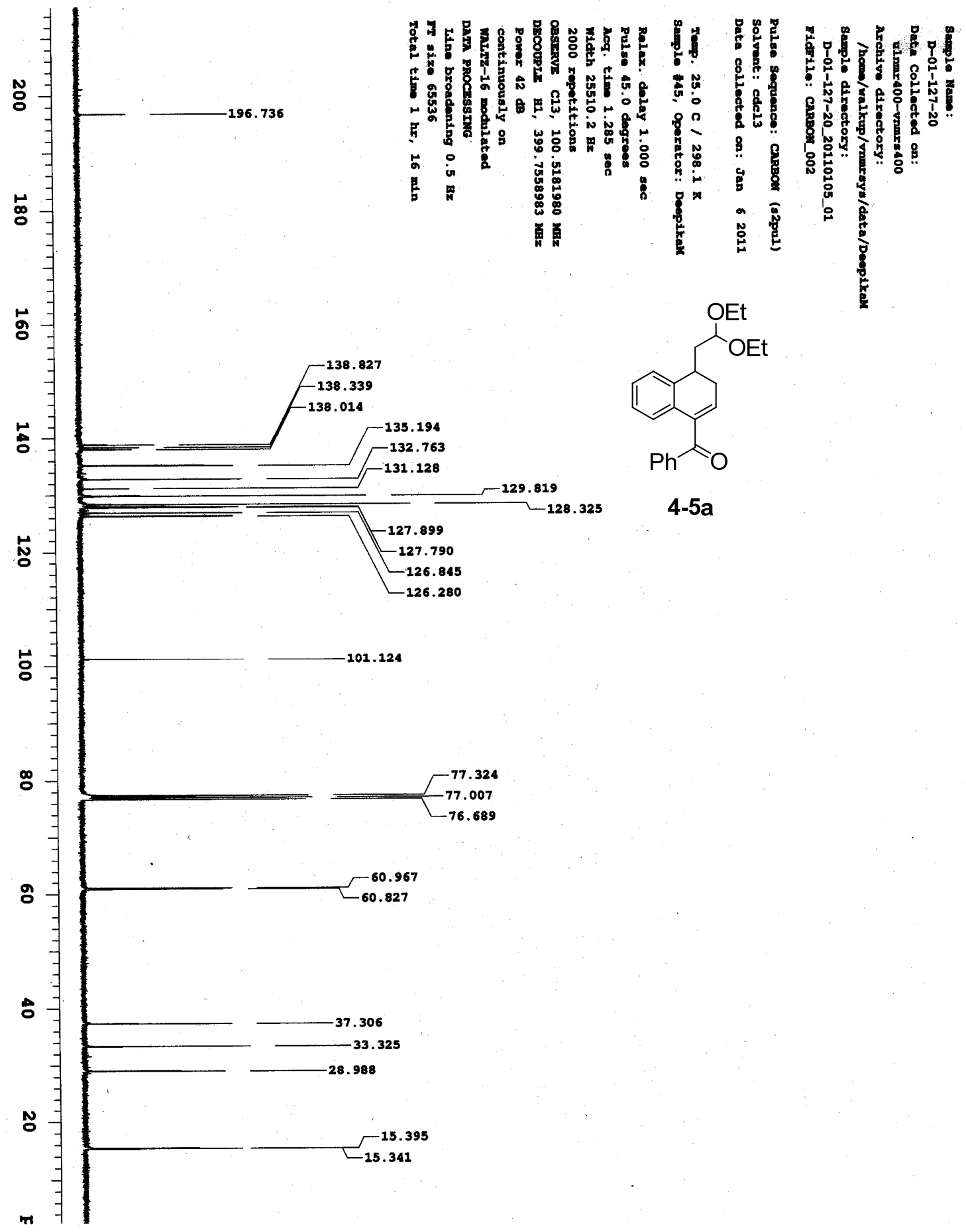




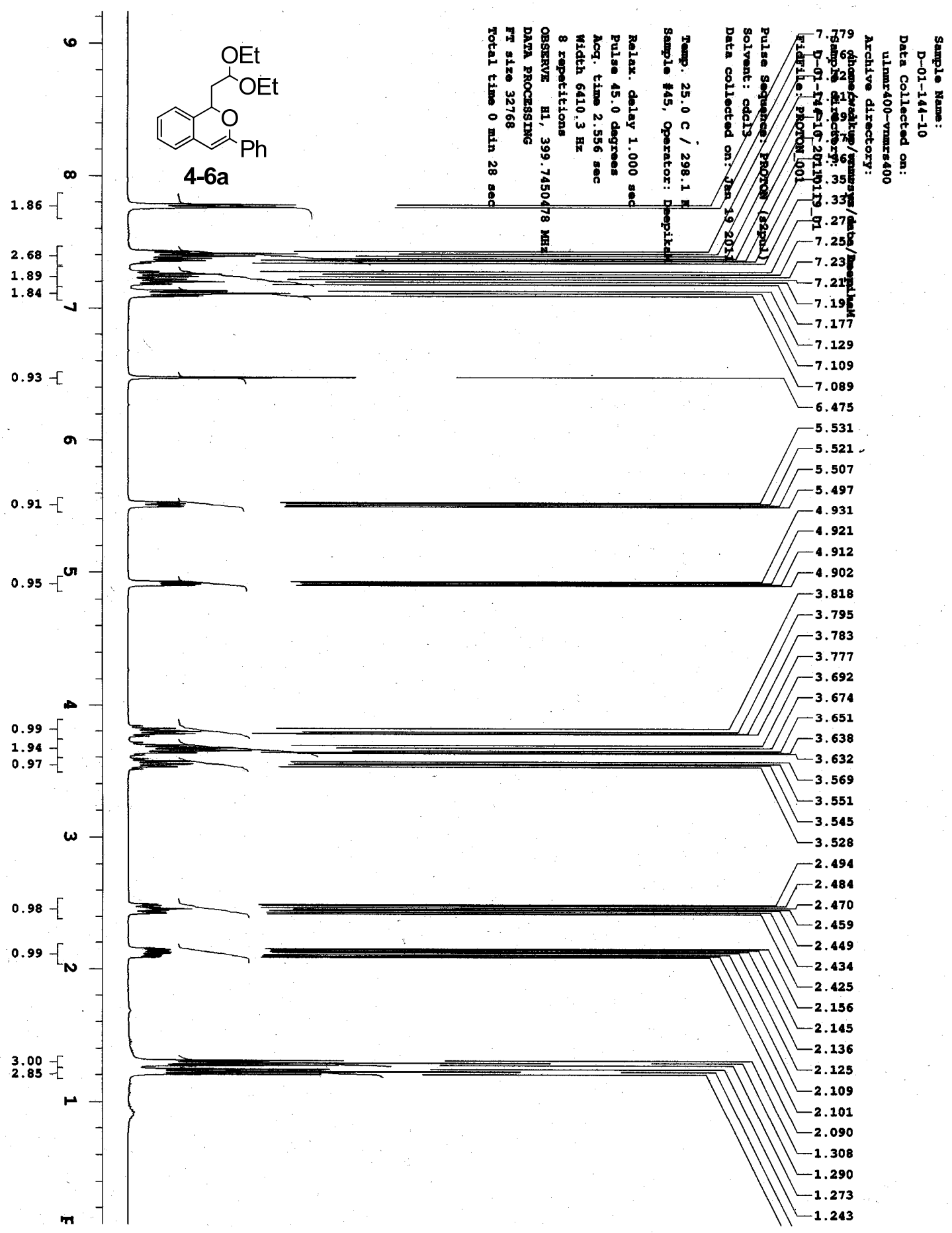




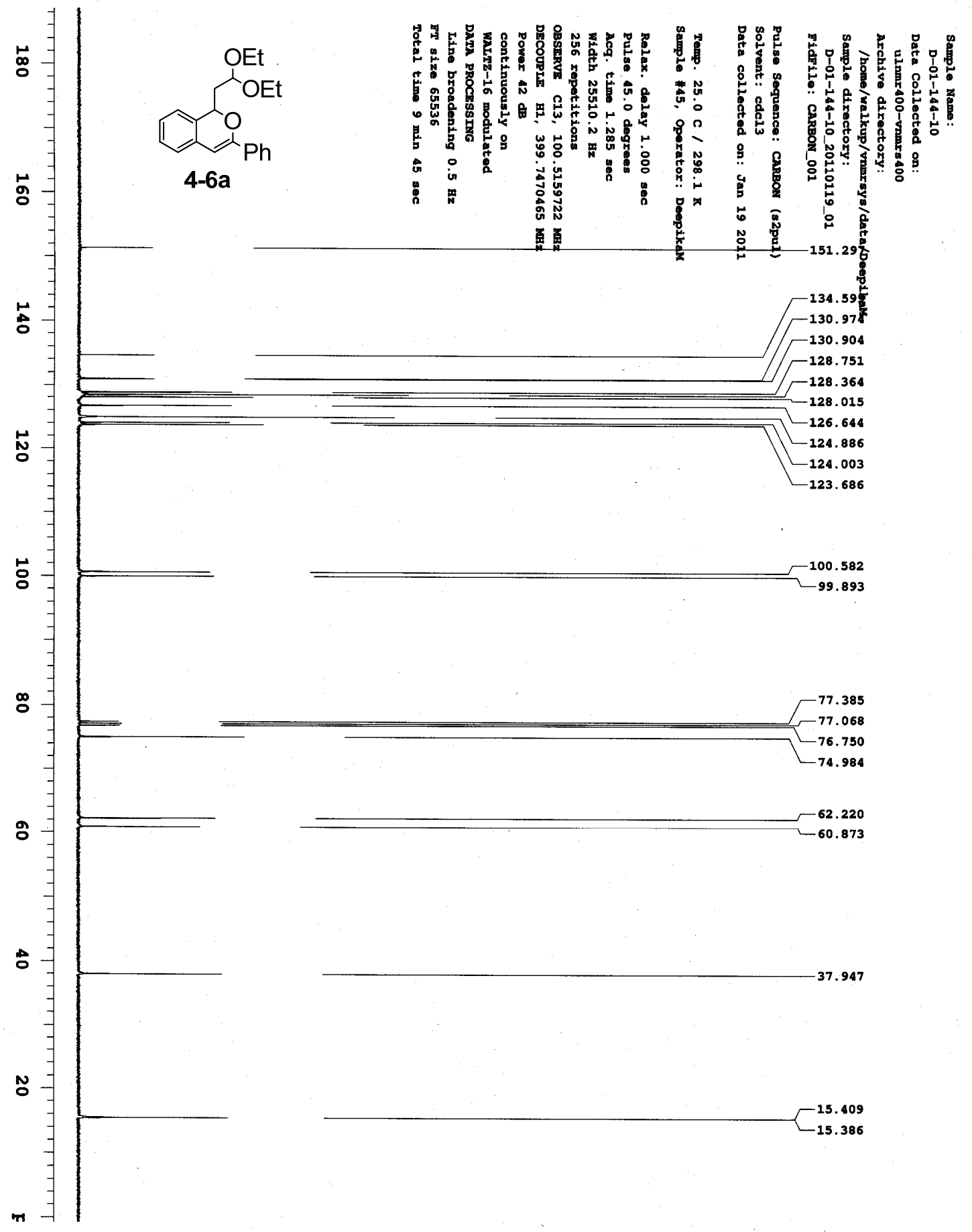



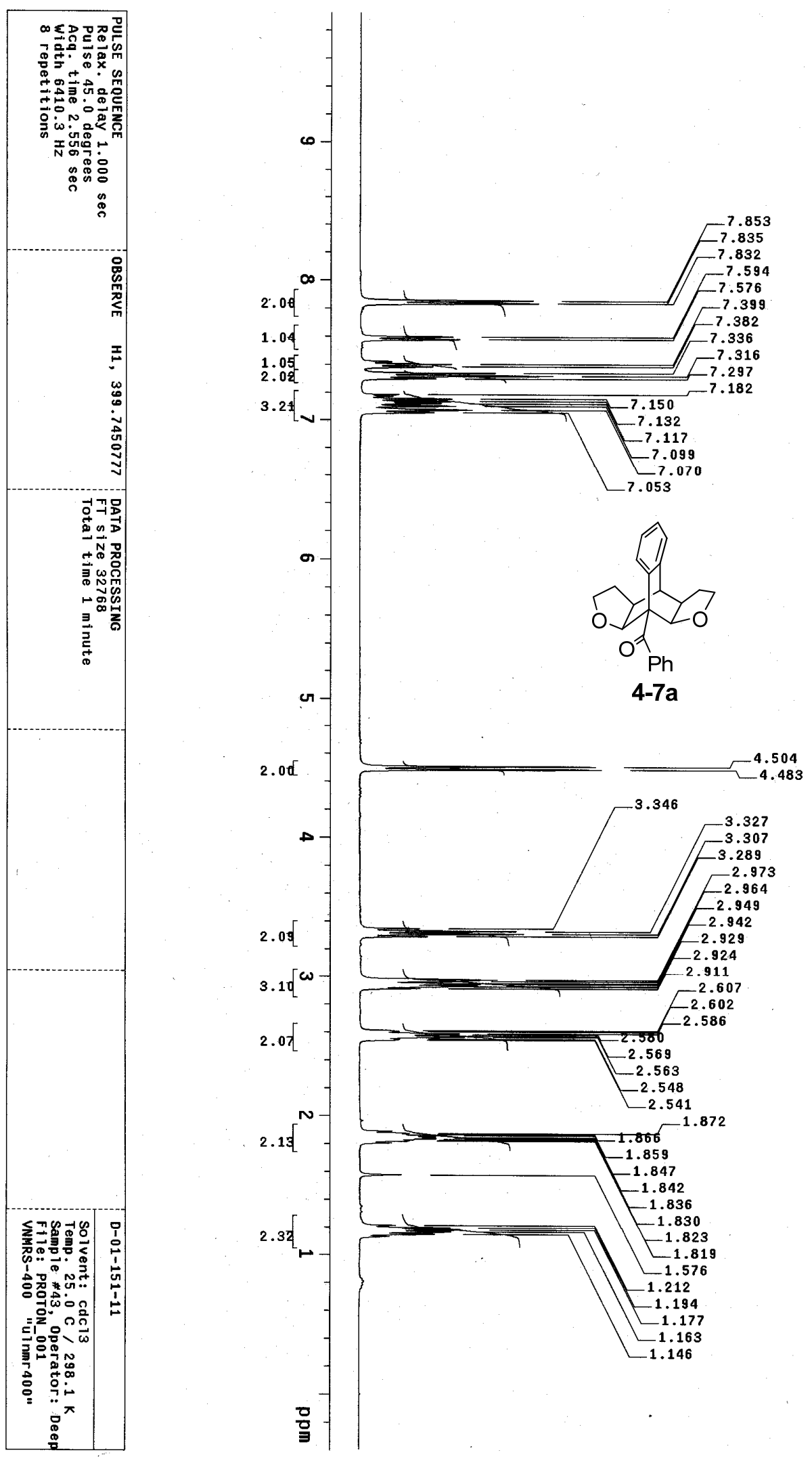


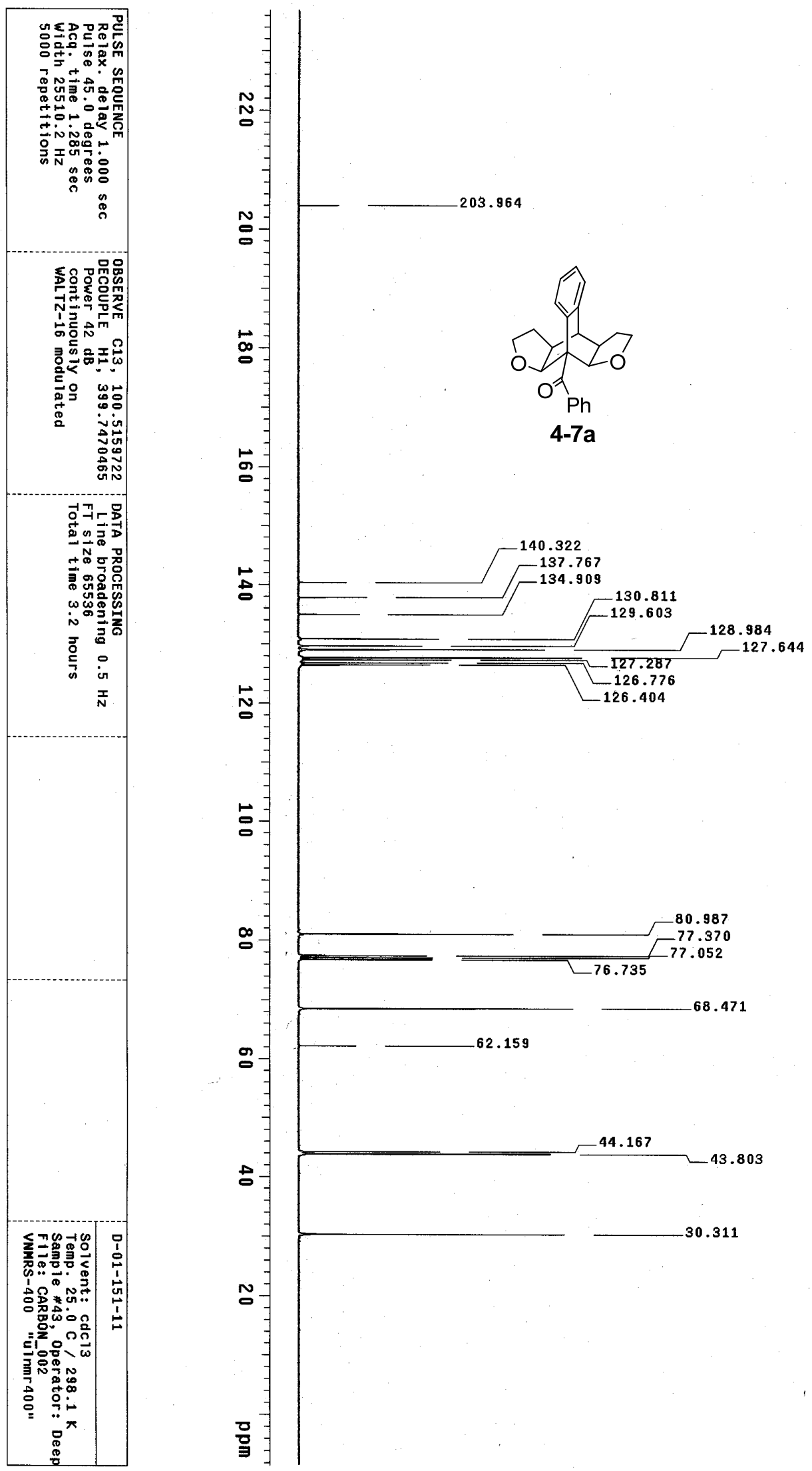



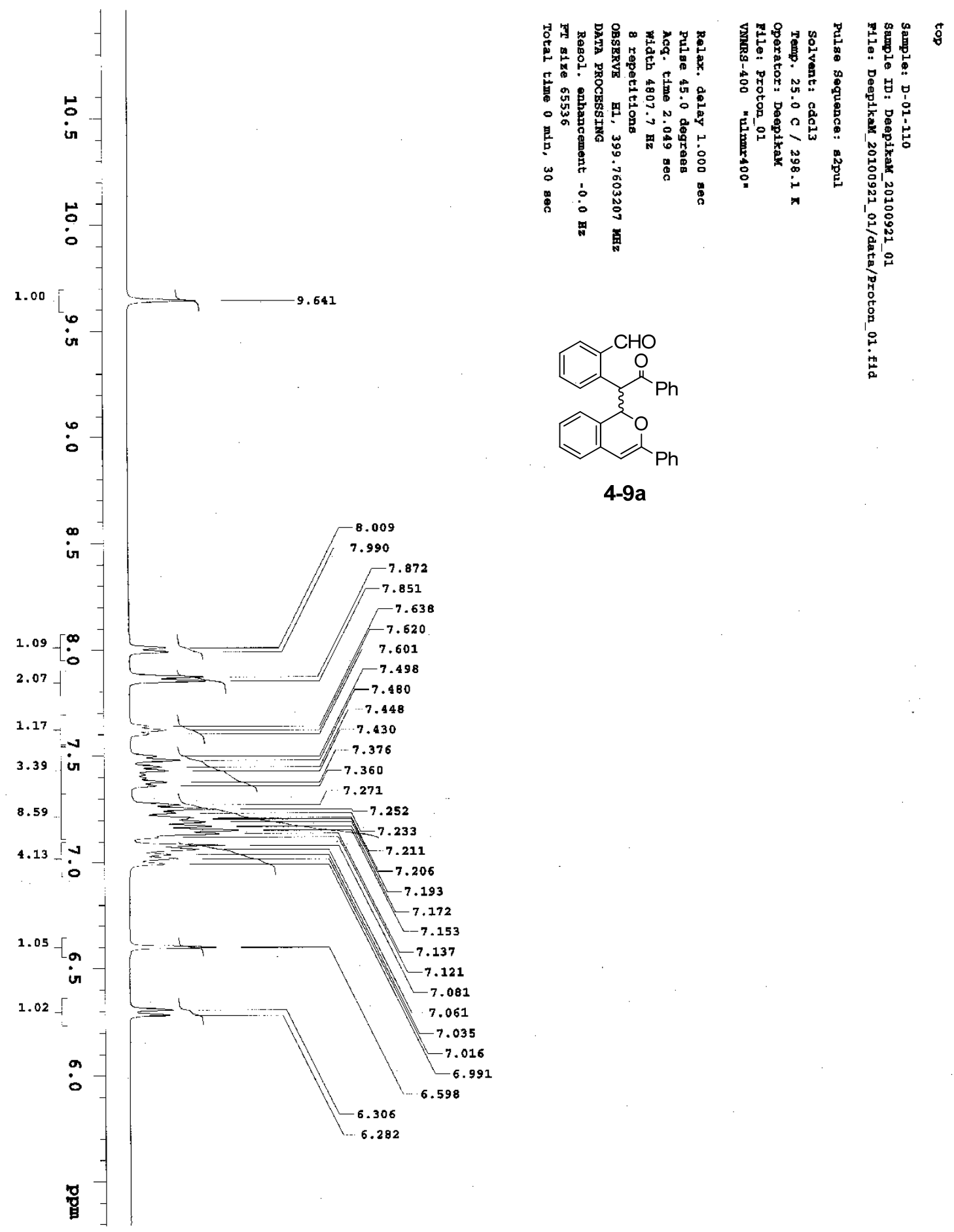


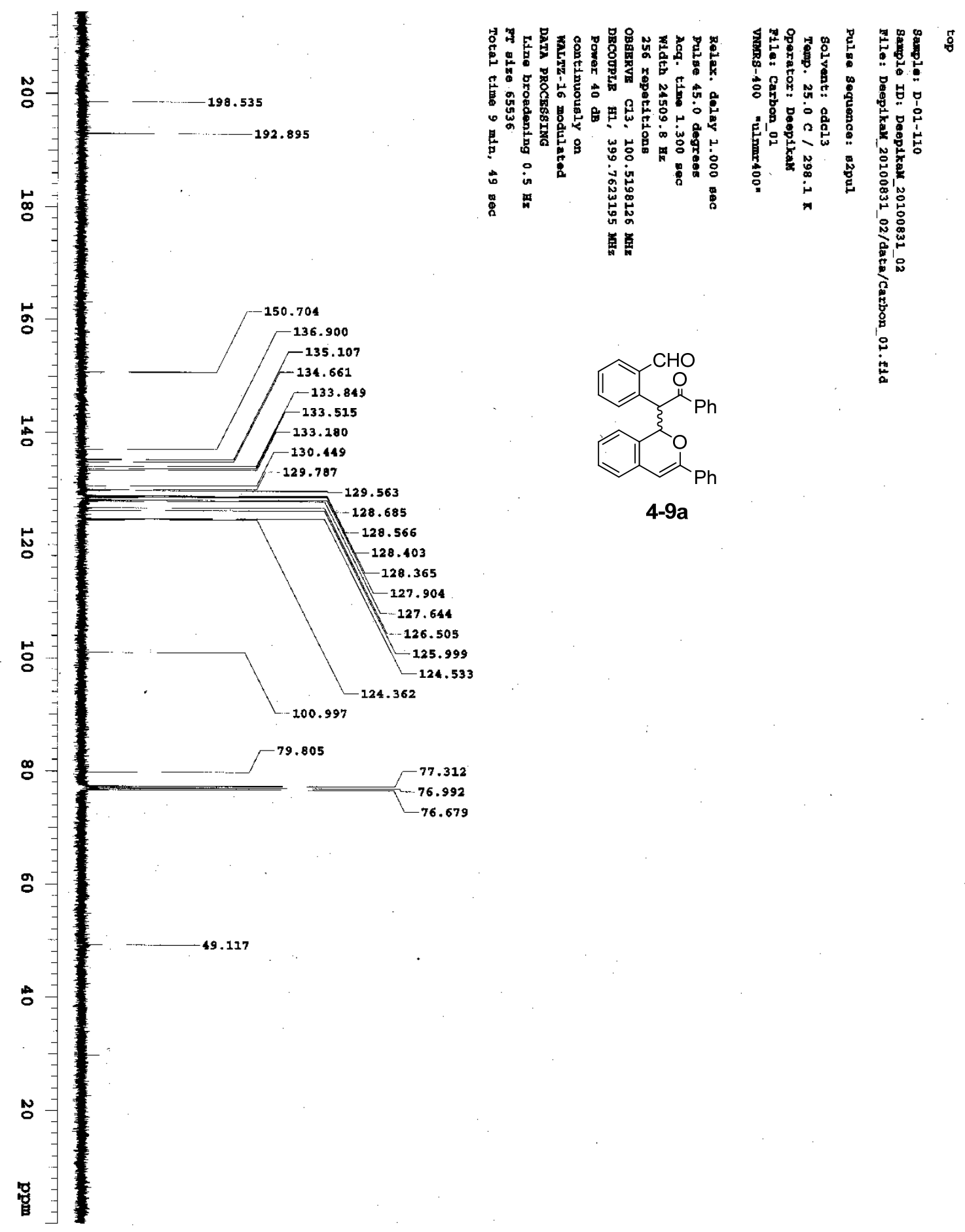




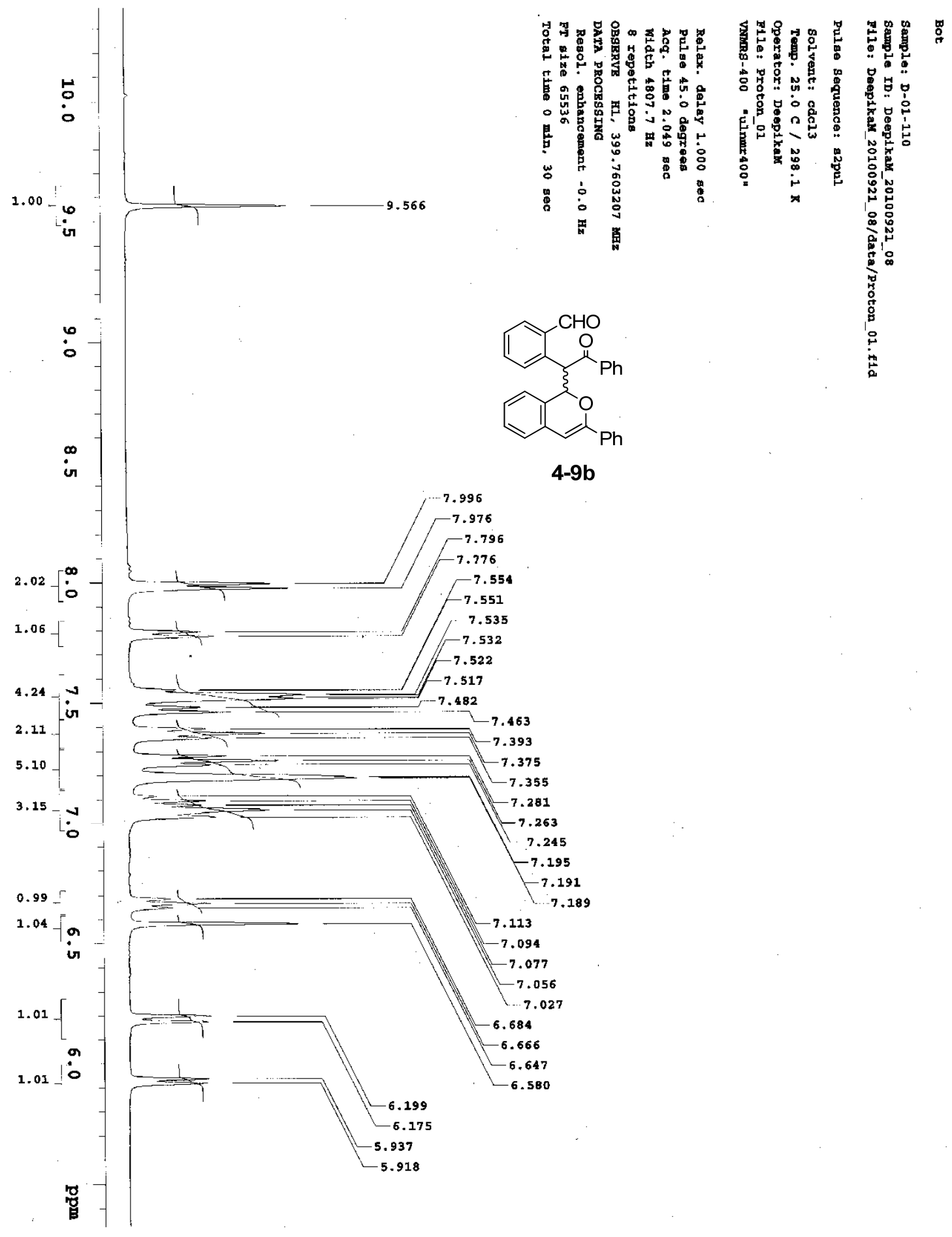




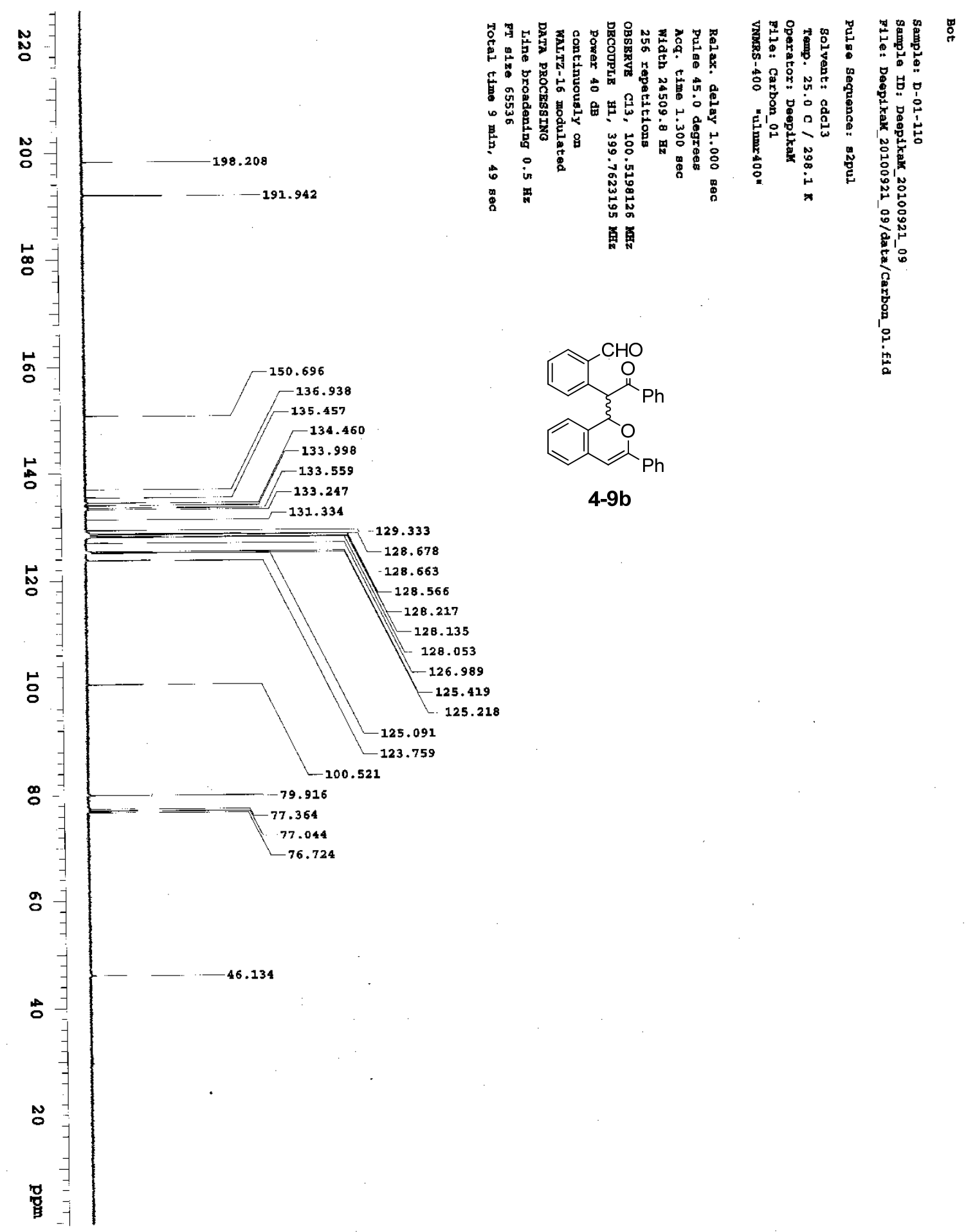




\section{CHAPTER 5. RATIONAL LIGAND DESIGN FOR GOLD CATALYSIS}

\subsection{Background}

Gold catalysis is a landmark addition to the field of organic synthesis., ${ }^{9,10,12,315-317}$ Cationic gold species are regarded as the most powerful catalysts for the electrophilic activation of C-C unsaturated compounds (alkynes/allenes/alkenes) toward a variety of nucleophiles. ${ }^{318-322}$ However, high turnover numbers (or low catalyst loadings) have been achieved only for a narrow set of gold-catalyzed reactions. ${ }^{323,324}$ Notable examples include the $\left[(\mathrm{NHC}) \mathrm{Au}^{\mathrm{I}}\right]$-catalyzed alkyne hydration, reported by Nolan and coworkers; ${ }^{325}$ the $\left[(\mathrm{NHC}) \mathrm{Au}^{\mathrm{I}}\right]$-catalyzed intramolecular addition of diol to alkyne, reported by Hashmi and coworkers; ${ }^{45}$ the hydroamination of alkynes with a hyperhalogenated carba-closododecaborate anionic ligand, reported by Lavallo and coworkers; ${ }^{43}$ and the ester assisted hydration of alkynes catalyzed by small gold clusters, reported by Corma and coworkers. ${ }^{326}$ In some exceptional cases, even higher turnovers can be achieved but at the cost of employing relatively high temperatures (e.g. $\left.120^{\circ} \mathrm{C}\right) .{ }^{325}$

Despite these impressive numbers, for most gold-catalyzed reactions, it is still common to see $1-5 \%$ catalyst loadings. Considering that gold is a precious metal and homogeneous catalysts are difficult to recycle, a 5\% loading is often not practical in large-scale synthesis. And more often than not, high temperatures are needed to achieve 
high turnover numbers, ${ }^{325}$ which is not suitable for the synthesis of complex target molecules.

Our goal is to develop an 'ideal' gold catalyst that meets the following criteria: i) High turnover (or low gold catalyst loading) under mild conditions such as room temperature, or slightly elevated temperatures $\left(\leq 50^{\circ} \mathrm{C}\right)$. ii) Applicable for a large group of goldcatalyzed reactions with predictable behavior. iii) Easily obtainable (commercially available or easily prepared in the laboratory).

\subsection{Results and discussions}

A simplified gold catalytic cycle is shown in Scheme 36. First, a cationic gold complex $\left(\mathbf{A u}^{+}\right)$acts as Lewis acids to activate C-C multiple bonds to form a $\pi$-complex Au-S. Interaction of $\mathbf{A u}-\mathbf{S}$ with a nucleophile $(\mathrm{Nu})$ eventually will give a gold $\sigma$-complex (AuS-Nu) (stage 1). The next stage is the regeneration of cationic gold (e.g. protodeauration), which gives the product $\mathbf{P}$ (stage 2). In addition, decay of cationic gold(I) complexes also takes place, leading to the formation of an inactive species like $\mathrm{Au}(0), \mathrm{L}_{2} \mathrm{Au}^{+}$(stage 3). Recently, Widenhoefer and coworkers reported the formation of reversible off-cycle gold $\operatorname{species}^{327}$ (e.g. bis-Au-vinyl species, stage 3). 


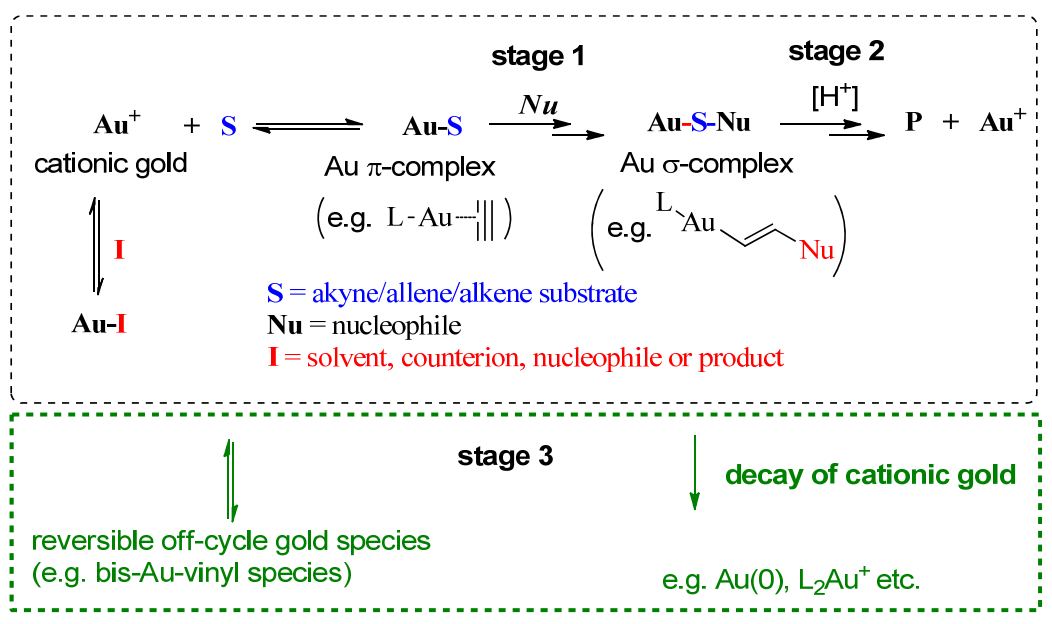

\section{Scheme 36. Type I and Type II gold-catalyzed reactions.}

Based on our most recent study, ${ }^{328}$ we can classify gold-catalyzed reactions based on their turnover limiting stage. According to Widenhoefer's recent report, ${ }^{329,330}$ the complexation of cationic gold with $\mathbf{S}$ is a fast process, so this step is unlikely to be ratedetermining. Therefore, we can classify most homogeneous gold-catalyzed reactions into two categories: type I, when formation of the gold- $\sigma$-complex (stage 1 in Scheme 36) is the turnover limiting step; and type II, when the regeneration of cationic gold catalyst (e.g. protodeauration) from gold- $\sigma$-complex (stage 2 ) is the turnover limiting stage.

Most gold-catalyzed reactions actually are type II. This usually occurs when the nucleophile is relatively reactive and the substrate is an alkyne. ${ }^{328}$ The turnover limiting stage of a type II reaction is the regeneration of a cationic gold catalyst. Because this process involves the generation of cationic gold, an electron rich ligand capable of supplying electron density to the gold metal center should facilitate this process. And because protodeauration is the most common path for cationic gold regeneration, we used the protodeauration of a preformed gold complex as our model for the evaluation of ligand effects (Table 9). 
For approximation purposes, the electron richness of a phosphine ligand was quantified using Tolman's parameter, $\Sigma \chi_{\mathrm{i}}\left(\mathrm{i}=1\right.$ to 3 ). ${ }^{331} \Sigma \chi_{\mathrm{i}}$ corresponds to the combined effect of three groups attached to a phosphine center. A smaller number signifies higher electron density on the phosphine ligand. From Table 9 we can observe that the rates of protodeauration directly correlate to $\Sigma \chi_{i}$ for ligands with similar structural motifs (e.g. para-substituted phenyl groups) (Table 9, entries 1-4). When we switched the methyl substituent from the para position to the ortho position (Table 9, entry 5), the rate of protodeauration decreased even though the electron density of the ligands in entries 2 and 5 should have been similar. This discrepancy could be explained by an increase in steric hindrance. But when we replaced one phenyl ring in $\mathrm{Ph}_{3} \mathrm{P}$ with an o-biphenyl group (Table 9, entry 6), the rate of protodeauration increased significantly, even though the electronic density was similar to that in entry 3 .

Table 9: Ligand effects in the protodeauration of vinyl gold complex 1.

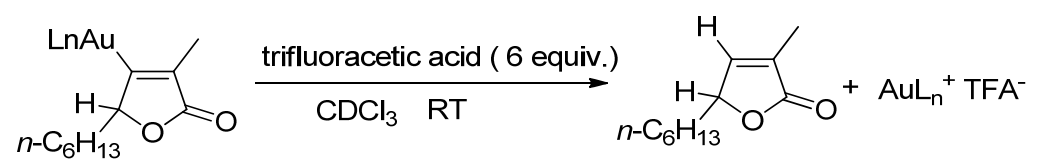

\begin{tabular}{|c|c|c|c|c|c|c|c|}
\hline & ligand & $\Sigma \chi_{\mathrm{i}}$ & $\begin{array}{l}\text { rel. } \\
\text { rate }\end{array}$ & & ligand & $\Sigma \chi_{\mathrm{i}}$ & $\begin{array}{l}\text { rel. } \\
\text { rate }\end{array}$ \\
\hline 1 & & 10.5 & 4.7 & 5 & & 10.8 & 1.3 \\
\hline 2 & & 10.8 & 2.2 & 6 & & 12.9 & 2.5 \\
\hline 3 & & 12.9 & 1.0 & 7 & & 4.3 & 31 \\
\hline 4 & & 15 & 0.16 & & & & \\
\hline
\end{tabular}


The increased steric hindrance should have reduced the rate of protodeauration, at least in theory. The combined effects of an $o$-biphenyl group and two electron donating groups ( $t$ $\mathrm{Bu}$ ) led to a very fast protodeauration (Table 9, entry 7).

The detailed mechanism for the ortho-aromatic ring substitution-enhanced protodeauration is not yet clear. Our hypothesis is that during protodeauration-the process that generates the cationic gold center-the ortho-phenyl ring may funnel small amounts of electronic density to the gold and therefore reduce the energy of the transition state (Figure 10a). Two notable findings are worth remarking: Widenhoefer and coworkers reported the formation of reversible off-cycle bis-Au-vinyl species, ${ }^{332-337}$ which are very resistant towards protodeauration; and Buchwald and coworkers' development of phosphine ligands with an o-biphenyl dialkylphosphine backbone (e.g., JohnPhos, XPhos), widely used in transition metal catalysis. ${ }^{338-342}$ With the above findings as preamble, we hypothesized that two sterically demanding biaryls on the phosphine ligand could surround the gold center further and discourage the formation of bis-Au-vinyl species (Figure 10b).
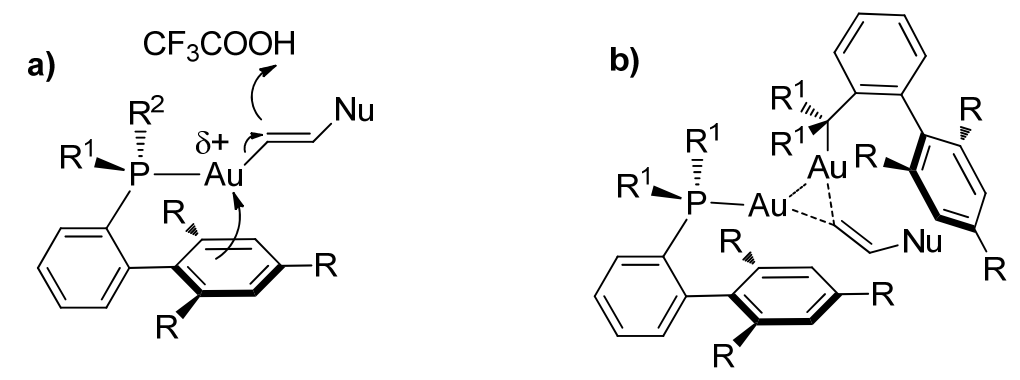

\section{Figure 10: o-Biphenyl group enhanced protodeauration.}

In order to achieve a fast turnover in a type II gold-catalyzed reaction, we proposed the following guidelines for ligand design: i) create an electron rich phosphine center; ii) use ortho-substitution to reduce catalyst deactivation and discourage the formation of off- 
cycle bis-Au-vinyl species; iii) incorporate the o-biphenyl motif on a phosphine ligand to reduce the deactivation of cationic gold. ${ }^{328}$ According to Tolman's phosphine ligand electronic effects study, ${ }^{331}$ the strongest electron donating groups in a phosphine ligand are substituted alkyl groups (e.g. t-Bu, Cy-). Hence, we will use these groups to create an electron rich phosphorus center. And because, in principle, we can introduce up to two obiphenyl motifs (or other ortho-substituted structures) on a phosphine ligand, we can define two categories of ligands (Figure 11). Category 1 corresponds to mono-biphenyl ligands (mainly Buchwald-type ligands, many of which are commercially available). Category 2 corresponds to bis-biphenyl ligands, which can be made from commercial available materials in one-step. ${ }^{343}$

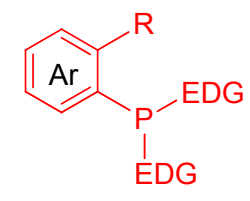

Category 1

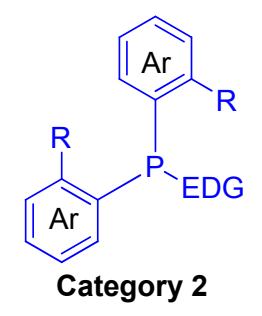

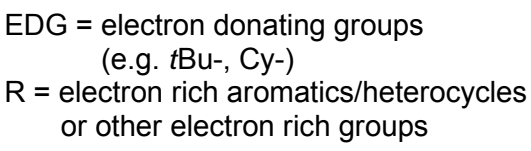

Figure 11. Two categories of phosphine ligands.

In the literature, electron rich ligands such as $N$-heterocyclic carbene-based ligands (e.g. Nolan and Hashmi's NHC ligands, Figure 12) have been used to achieve high turnover numbers. ${ }^{344,345}$ They all have a steric handle near the gold center. The success of these NHC ligands may be due to their electron rich nature and their steric handles. For our study though, we selected phosphine-based ligands because of their relatively easy synthesis and the ease by which we could manipulate their electron density and steric effects. 


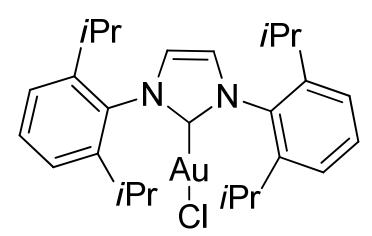

IPr-Au-Cl

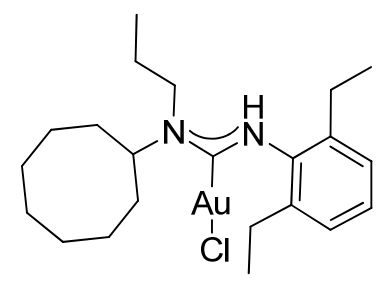

Figure 12. N-Heterocyclic carbene-based gold pre-catalysts. ${ }^{4,5}$

Thus, we designed ligand L1 (Figure 13) featuring two electron-rich and sterically demanding ortho-biphenyl groups and one electron rich cyclohexyl group. We prepared $\mathbf{L 1}$ in a single step from commercially available starting materials ${ }^{343}$ (shown in experimental section). The crystallographic structure of $\mathrm{L} 1-\mathrm{AuCl}$ (Figure 13) ${ }^{346}$ demonstrated that the two ortho-biphenyl motifs were able to surround or embed the gold center.

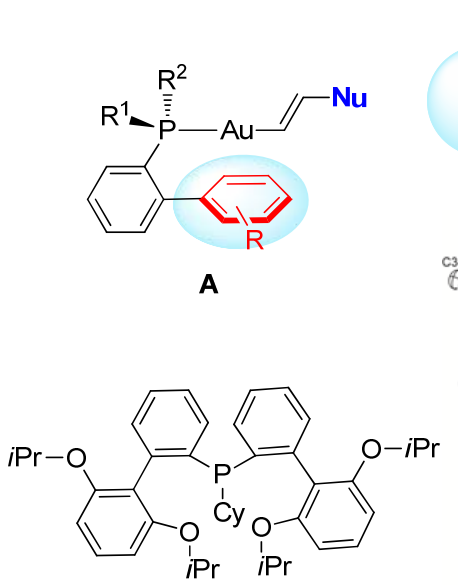

L1

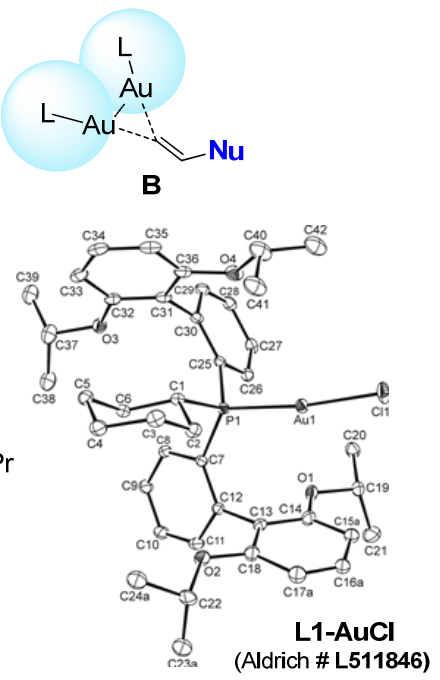

Figure 13. Ligand design for gold catalysis.

A comparison between our new ligand L1 and other benchmark ligands is shown in Table 10. We chose the carbene-based gold catalyst $\mathbf{L} \mathbf{4}$ as a yardstick because of its proven efficiency towards gold-catalyzed reactions and the progress of these reactions was monitored by NMR experiment array. The details have been described in the experimental section. $\mathbf{L} \mathbf{1}$ was the most reactive ligand (Table 10). A ligand with three $o$ - 
biphenyl motifs (L14) was less effective, probably because of its lower electronic density. The importance of the ligand electronic density on the rate of hydroamination became apparent when we kept the o-biphenyl motif unchanged and modulated the electron densities of the two remaining groups connected to phosphorus ( $\mathbf{L 5}$ to $\mathbf{L 8}$ ). In this subset, electron richer ligands produced faster reaction rates. Substituting the $o$ biphenyl group with ferrocene decreased the reaction rate significantly (Table 10, $\mathbf{L 8}$ and L9). The merits of $\mathbf{L 1}$ were further assessed in other gold-catalyzed intermolecular and intramolecular reactions with $\mathrm{C}, \mathrm{N}$, or $\mathrm{O}$ nucleophiles so as to determine the reaction conditions needed to achieve the highest possible turnover for each of the reactions tested.

Table 10. Relative rates of hydroamination for various ligands.

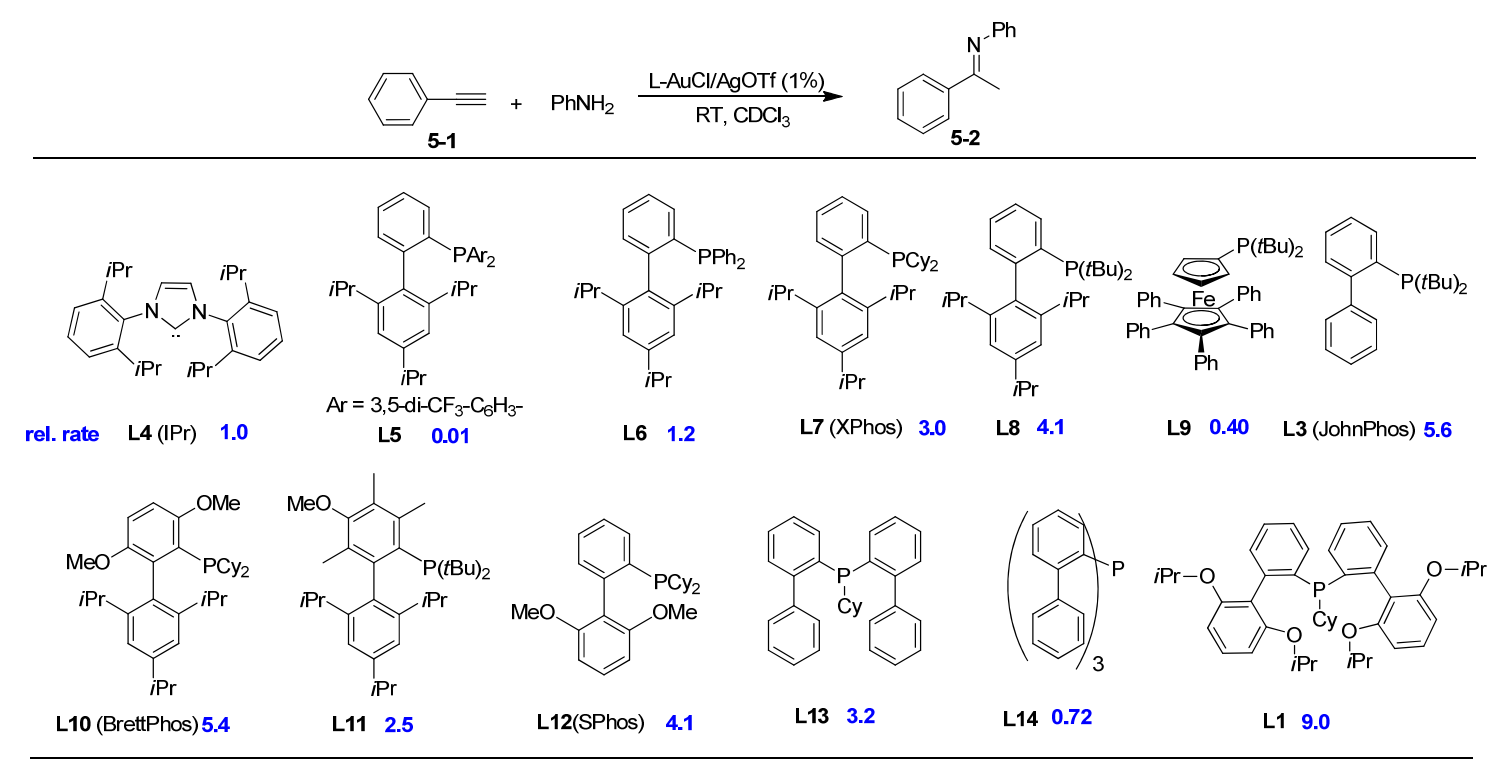

We revisited the hydroamination reaction because it is a representative example of a $\mathrm{C}-\mathrm{N}$ bond forming reaction where nitrogen is the nucleophile. Tanaka and coworkers reported an efficient gold-catalyzed intermolecular hydroamination of alkynes using the precatalyst $\mathrm{PPh}_{3} \mathrm{AuCH}_{3}$ in the presence of $\mathrm{H}_{3} \mathrm{PW}_{12} \mathrm{O}_{40}$ acting as acidic promoter. ${ }^{347}$ Using 
an acidic promoter is appropriate in hydroamination because protodeauration is the rate limiting step. $^{328}$ Shi and coworkers reported an even higher turnover using a $\mathrm{Ph}_{3} \mathrm{PAuOTf} /$ benzotriazole/ $\mathrm{H}_{3} \mathrm{PW}_{12} \mathrm{O}_{40}$ system (Scheme $\left.37-\mathrm{a}\right) .{ }^{348}$ When we used Tanaka's acidic promoter with our $\mathrm{L} 1-\mathrm{AuCl}$ precatalyst and the alkali salt of the bulky counterion $\mathrm{CTf}_{3}{ }^{-}$, we found that the catalytic loading could be reduced to $0.0025 \%$ (25 ppm) and the reaction reached an impressive TON of 31200. To the best of our knowledge, no other catalyst system has matched this prowess at such low temperature $\left(50^{\circ} \mathrm{C}\right)$. Our catalyst system also worked equally well in relatively larger scales $(10 \mathrm{mmol})$.

We chose the gold-catalyzed intermolecular addition of N-hydroxyl benzotriazole 5-4 to an alkyne as our model system for O-H addition. ${ }^{349}$ We were able to reduce the catalytic loading to $0.1 \%$ (Scheme $37-b$ ). This result is a significant improvement over the original literature report (Scheme 37-b), which needed 5\% catalyst loading.

We also tested the intramolecular version of $\mathrm{X}-\mathrm{H}$ addition to alkyne which is, in general, more efficient than the intermolecular version. For example, in the gold-catalyzed cyclization of homopropargylic diols, ${ }^{350,351}$ we observed that $\mathrm{L} 1-\mathrm{AuCl}$ needed only 20 ppm to yield $92 \%$ of product 5-7 after $24 \mathrm{~h}$ at room temperature (Scheme 37 -c). This result was significantly better than the $2 \%$ catalyst loading cited in the literature. ${ }^{350,351}$ $\mathrm{L} 1-\mathrm{AuCl}$ was also efficient in the intramolecular cyclization of 4-pentynoic acid 5-8 (Scheme 37-d), as demonstrated by the TON of 9900 achieved at room temperature. This result was far better than the available literature report (Scheme 37-d). ${ }^{352,353}$ 
a)

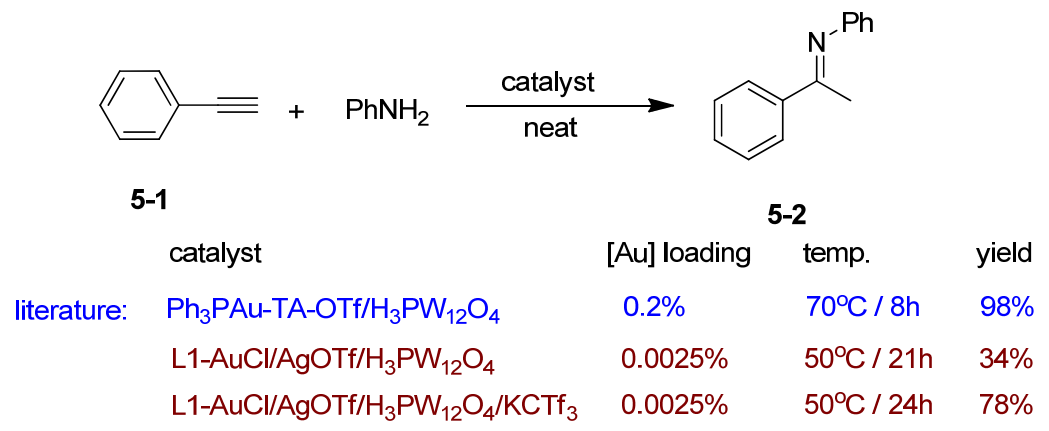

b)

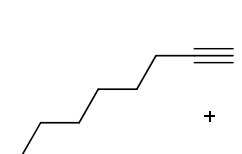

5-3

catalyst

literature: $\quad \mathrm{Ph}_{3} \mathrm{PAuCl} / \mathrm{AgOTf}$

L1-AuCl/AgOTf

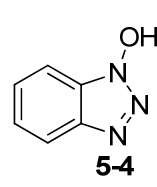

$5-4$

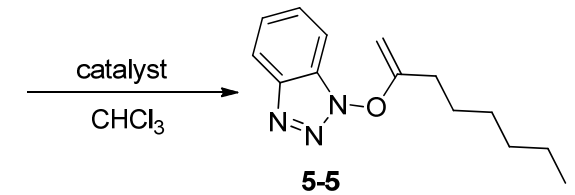

5-5

[Au] loading temp. yield

$5 \% \quad$ RT $/ 12$ h $\quad 94 \%$

$0.1 \% \quad$ RT $/ 24 \mathrm{~h} \quad 95 \%$

c)

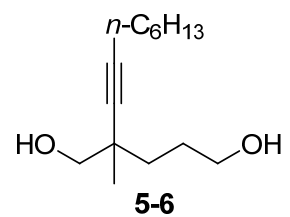

catalyst

literature: $\mathrm{AuCl}$

L1-AuCl/AgSbF 6

$\mathrm{L} 1-\mathrm{AuCl}_{\mathrm{AgSbF}} / \mathrm{KCTf}_{3}$

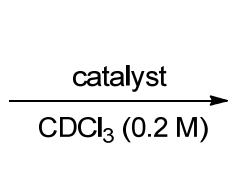

[Au] loading temp. yield

$2 \%$

$0.002 \% \quad \mathrm{RT} / 24 \mathrm{~h} \quad 24 \%$

$0.002 \%$
$n-\mathrm{C}_{6} \mathrm{H}_{13}$

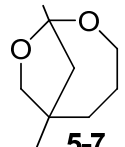

RT/0.2 h $\quad 91 \%$

RT / 24 h $92 \%$

d)
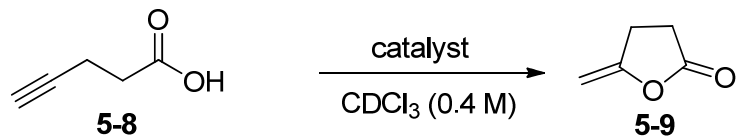

catalyst

literature: $\quad \mathrm{Au}_{2} \mathrm{O}_{3}$

[Au] loading temp. yield

L1-AuCl/AgOTf/KCTf 3

$0.5 \%$

RT / 2 h $\quad 99 \%$

$0.01 \%$

RT / $1 \mathrm{~h} \quad 99 \%$

\section{Scheme 37. Use of $\mathrm{L1-AuCl}$ in $\mathrm{X}-\mathrm{H}(\mathrm{X}=\mathrm{N}, \mathrm{O})$ addition to alkynes.}

Since C-C bond formation is the most important class of reactions in synthesis, we studied several gold-catalyzed $\mathrm{C}-\mathrm{H}$ additions to alkynes. We evaluated the Conia-ene reaction of $\beta$-ketoester 5-10 first $^{354}$ (Scheme 38-a). Again, L1-AuCl worked very well in this reaction: it needed only $0.004 \%$ (40 ppm) catalyst loading at room temperature to 
furnish the product in $95 \%$ yield. The use of an acidic promoter enhanced the reactivity, just as in the case in the hydroamination reaction described earlier.

a)

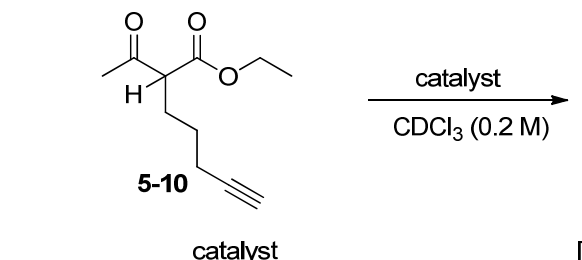

$$
\text { literature: }
$$

$\mathrm{Ph}_{3} \mathrm{PAuCl} / \mathrm{AgOTf}$

L1-AuCl/AgOTf/HOTf

L1-AuCI/AgOTf/HOTf

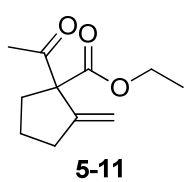

[Au] loading temp. yield

$1 \% \quad$ rt $/ 0.1 \mathrm{~h} \quad 94 \%$

$0.01 \% \quad$ RT $/ 1 \mathrm{~h} \quad 95 \%$

$0.004 \%$ RT / $28 \mathrm{~h} \quad 95 \%$

b)

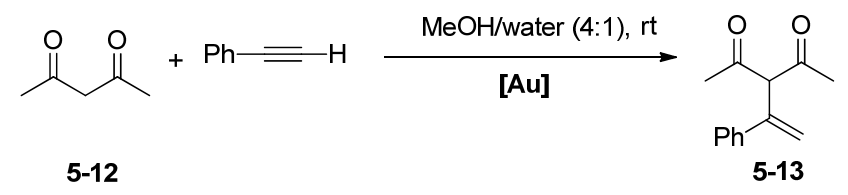

$\begin{array}{ll} & \text { catalyst } \\ \text { literature: } & \mathrm{Ph}_{3} \mathrm{PAuCl} / \mathrm{AgOTf} \\ & \mathrm{L} 1-\mathrm{AuCl} / \mathrm{Ga}(\mathrm{OTf})_{3} \\ & \mathrm{~L} 1-\mathrm{AuCl} / \mathrm{Ga}(\mathrm{OTf})_{3}\end{array}$

$\begin{array}{lll}\text { [Au] loading } & \text { temp. } & \text { yield } \\ 1 \% & \mathrm{RT} / 17 \mathrm{~h} & <5 \% \\ 0.05 \% & 45^{\circ} \mathrm{C} / 18 \mathrm{~h} & 91 \% \\ 0.025 \% & 45^{\circ} \mathrm{C} / 96 \mathrm{~h} & 76 \%\end{array}$

c)
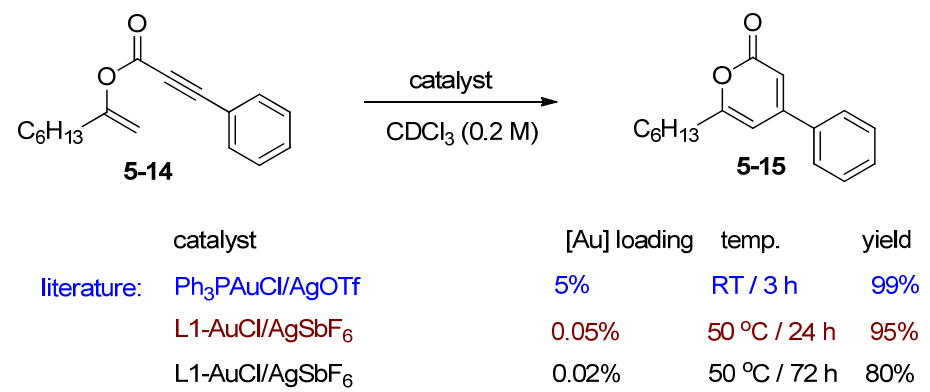

d)
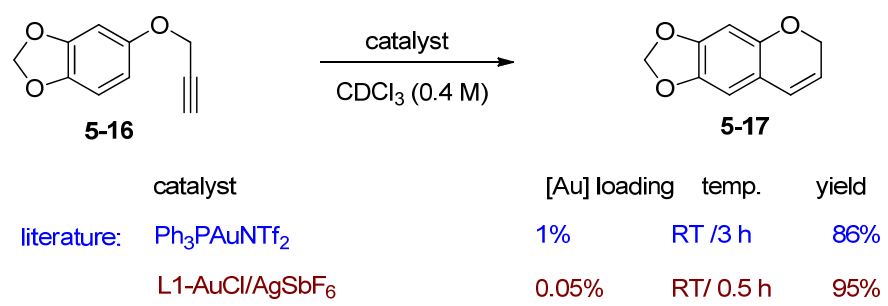

Scheme 38. C-H additions to alkynes (C-C bond formations).

$\mathrm{L} 1-\mathrm{AuCl}$ also worked well in the intermolecular version of the Conia-ene reaction (Scheme 38-b); very good to excellent yields could be achieved in this reaction using low catalyst loadings in the presence of a co-catalyst-Ga(OTf) $)_{3}{ }^{355-357} \mathrm{~L} 1-\mathrm{AuCl}$ also 
performed efficiently in the synthesis of $\alpha$-pyrone (Scheme 38-c). We obtained the pyrone product 5-15 in $95 \%$ yield at $50^{\circ} \mathrm{C}$ using a catalyst load of only $0.05 \%$ (500 ppm) whereas the corresponding reaction cited in the literature ${ }^{358}$ needed a $5 \%$ loading. ${ }^{359} \mathrm{We}$ reduced the catalyst loading to $0.02 \%(200 \mathrm{ppm})$ and still managed to obtain a respectable $80 \%$ yield (Scheme 38c). To broaden the applicability of our gold catalyst $\mathrm{L} 1-\mathrm{AuCl}$, we chose another example of a $\mathrm{C}-\mathrm{H}$ addition to an alkyne, namely the hydroarylation of 5-16 ${ }^{360}$ (Scheme 38-d). The product, $2 \mathrm{H}$-chromene 5-17, was obtained in $95 \%$ yield at room temperature using a catalyst loading of $0.05 \%$ (500 ppm).

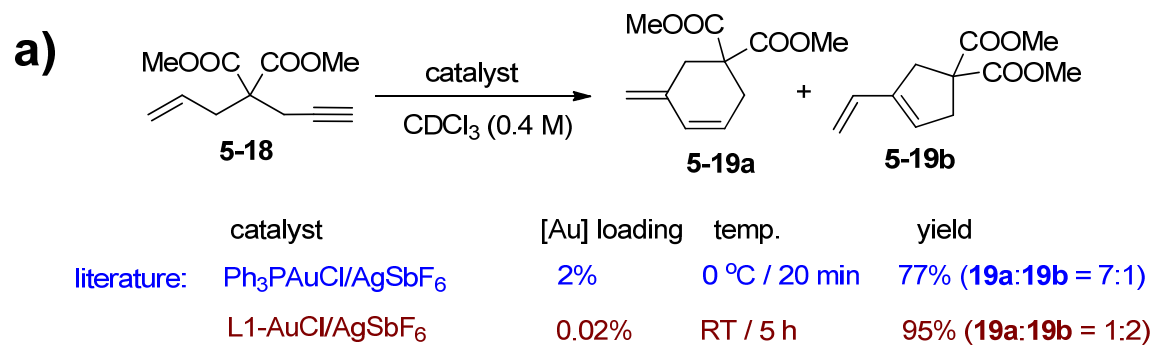

b)

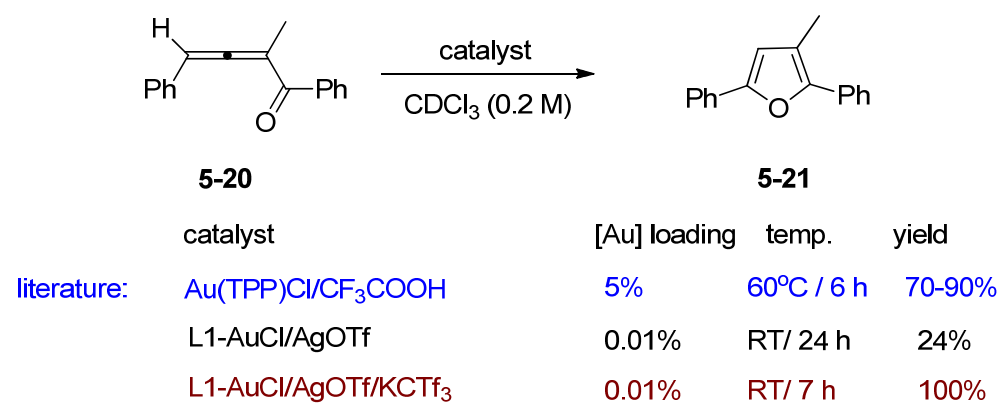

Scheme 39. Enyne and allenone cycloisomerization.

Enyne cycloisomerization is a class of reactions where gold catalysis has proved its might. ${ }^{361-363}$ Using 1,6-enyne 5-18 as model substrate, we found that our gold catalyst needed only a loading of $0.02 \%$ (200 ppm) to drive the enyne cycloisomerization to completion, furnishing 5-19 in almost quantitative yield (Scheme 39-a). In contrast, a similar reaction reported in the literature $e^{361-363}$ needed a $2 \%$ catalyst loading. We also investigated the cycloisomerization of allenone 5-20, first reported by Hashmi and 
coworkers $^{364}$ and later refined by Che and coworkers (Scheme 39-b) ${ }^{365}$ Using $0.01 \%$ (100 ppm) of our catalyst $\mathrm{L} 1-\mathrm{AuCl}$, we obtained the desired furan 5-21 in quantitative yield after $7 \mathrm{~h}$ at room temperature. This result corresponded to a TON of 10,000 .

\subsection{Conclusions}

In summary, we have developed a highly efficient and broadly applicable cationic gold catalyst system that is highly efficient at extremely low loadings and relatively low temperatures. The optimal catalyst $(\mathrm{L} 1 \mathrm{AuCl})$ is now available from Aldrich under the name BisPhePhos XD gold(I) chloride (catalog no. L511846). This new gold catalyst gives high turnover numbers (or low gold catalyst loading) that are in the ppm range, at room temperature or slightly elevated temperatures $\left(\leq 50^{\circ} \mathrm{C}\right)$. These results should help to turn gold catalysis into the tool of choice for larger-scale synthesis. The work described in this chapter is in press Angew. Chem. Int. Ed. 2014, 53, 4456-4459.

\subsection{Experimental}

\section{General}

${ }^{1} \mathrm{H}$ and ${ }^{13} \mathrm{C}$ NMR spectra were recorded at $500 \mathrm{MHz}$ and $126 \mathrm{MHz}$ (or $400 \mathrm{MHz}$ and 101 $\mathrm{MHz}$ ) respectively, using $\mathrm{CDCl}_{3}$ as a solvent. The chemical shifts are reported in $\delta(\mathrm{ppm})$ values $\left({ }^{1} \mathrm{H}\right.$ and ${ }^{13} \mathrm{C}$ NMR relative to $\mathrm{CHCl}_{3}, \delta 7.26$ ppm for ${ }^{1} \mathrm{H}$ NMR and $\delta 77.0 \mathrm{ppm}$ for

${ }^{13} \mathrm{C}$ NMR and $\mathrm{CFCl}_{3}\left(\delta 0 \mathrm{ppm}\right.$ for ${ }^{19} \mathrm{~F}$ NMR), multiplicities are indicated by s (singlet), $\mathrm{d}$ (doublet), t (triplet), q (quartet), p (pentet), h (hextet), m (multiplet) and br (broad). Coupling constants $(J)$, are reported in Hertz $(\mathrm{Hz})$. All reagents and solvents were employed without further purification. The products were purified using a commercial 
flash chromatography system or a regular glass column. TLC was developed on silica gel 60 F254 aluminum sheets. KCTf 3 was purchased from Synquest Labs. All other chemicals like metal catalysts and ligands were purchased from Aldrich, Alfa Aesar or Strem.

\section{General procedure for the synthesis of Ligand L1}

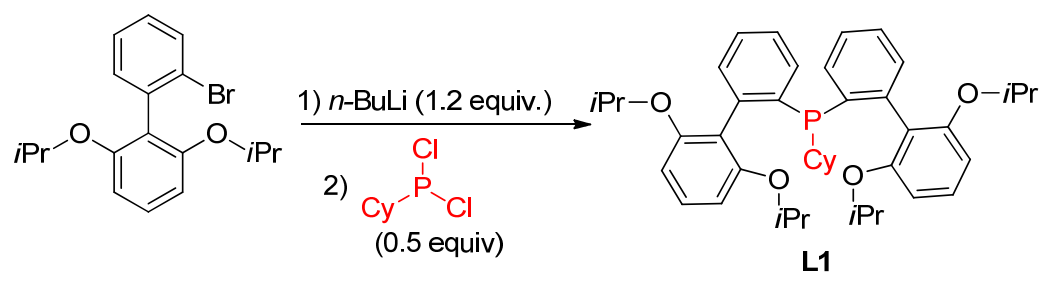

Under argon, diethyl ether $(140 \mathrm{~mL})$ was added to a reaction flask followed by the addition of 2-bromo-2',6'-diisopropoxy-1,1'-biphenyl (11.08 $\mathrm{mL}, 38.8 \mathrm{mmol})$. The flask was cooled in an ice bath, $n$-butyllithium $(20 \mathrm{~mL}, 2.5 \mathrm{M}, 50 \mathrm{mmol})$ was added slowly and the reaction mixture was stirred for 30 minutes at $0^{\circ} \mathrm{C}$. Dichlorophosphane $(3.0 \mathrm{~mL}, 19.4$ mmol) was added to the reaction mixture slowly and the mixture was warmed to room temperature and left stirring at room temperature for $48 \mathrm{~h}$. The reaction was quenched with water and was extracted by diethyl ether $(2 \times 100 \mathrm{~mL})$. The combined organic layer was dried on sodium sulphate. The solvent was evaporated to yield a viscous oil which was further purified by column chromatography using hexane:ethyl acetate (100:1) solvent system to yield semisolid which was recrystallized/triturated with methanol to yield a white solid 3.9 gram (30\%). 


\section{Synthesis of of gold complexes ( $\mathrm{L}-\mathrm{AuCl})$}

All gold complexes ( $\mathrm{L}-\mathrm{AuCl})$ were synthesized using a slightly modified version of a

literature method. ${ }^{247,366}$ These complexes were prepared via either one of the following general procedures:

Method 1: Sodium tetrachloroaurate(III) dihydrate $(1 \mathrm{mmol})$ was dissolved in water, and the orange solution was cooled in ice. To this solution, 2,2'-thiodiethanol (3 mmol) was slowly added (ca. $10 \mathrm{~min}$ ) with stirring. After stirring at $0^{\circ} \mathrm{C}$ for another $30 \mathrm{~min}$, a solution of the phosphine ligand (1 mmol) in $\mathrm{EtOH}$ (if the ligand could not be dissolved, more EtOH was used) was added dropwise to yield a white solid. The solid was filtered off, washed with water followed by EtOH, and ultimately dried in vacuum.

Method 2: In a vial Chloro(dimethylsulfide)gold(I) (1 $\mathrm{mmol})$ was dissolved in dichloromethane and cooled in an ice bath. A solution of phosphine ligand $(1 \mathrm{mmol})$ in dichloromethane was added dropwise, and the resulting solution was allowed to warm to room temperature and stirred at room temperature for $3 \mathrm{~h}$. After TLC indicated complete consumption of the starting material, the reaction solution was concentrated to dryness under reduced pressure, and the gold complex product was further dried under high vacuum.

\section{General procedures for model reactions}

\section{Preparation of stock solutions}

Because our gold precatalyst and silver activators were used at very low loading, we prepared the corresponding stock solutions. 


\section{Preparation of cationic gold ( $\mathrm{L}-\mathrm{AuCl})$ stock solution.}

Stock solution of $\mathrm{L}-\mathrm{AuCl}(0.01 \mathrm{M})$ was prepared by dissolving $\mathrm{L}-\mathrm{AuCl}(0.02 \mathrm{mmol})$ in $\mathrm{CDCl}_{3}(2 \mathrm{~mL})$. The solution was kept in the freezer $\left(-20^{\circ} \mathrm{C}\right)$ until it was needed.

\section{Preparation of stock solutions of silver activators (AgOTf and $\mathrm{AgSbF}_{6}$ ).}

Stock solutions of silver triflate and silver hexafluoroantimonate $(0.01 \mathrm{M})$ were prepared by weighing AgOTf or $\mathrm{AgSbF}_{6}(0.02 \mathrm{mmol})$ in a glass vial and dissolved it in deuterated acetone $(2 \mathrm{~mL})$. The solution was kept in the freezer $\left(-20^{\circ} \mathrm{C}\right)$ until it was needed.

\section{Preparation of cationic gold $\left(\mathrm{L}-\mathrm{Au}^{+} \mathrm{X}^{-}\right)$stock solution.}

Standard stock solutions of cationic gold catalyst were prepared by weighing the L-AuCl complex $(0.02 \mathrm{mmol})$ in a vial and adding the corresponding deuterated solvent (usually $\left.\mathrm{CDCl}_{3}\right)(2 \mathrm{~mL})$, followed by 1.2 equiv. of silver activator. The vial was sonicated for 5 10 min at $5-10{ }^{\circ} \mathrm{C}$, then it was centrifuged and the clear solution was transferred to a clean glass vial with a screw cap. The solution was kept in freezer $\left(-20^{\circ} \mathrm{C}\right)$ until it was needed.

\section{General procedure for the formation of imine (5-2)}

AgOTf stock solution ( $60 \mu \mathrm{L}, 0.01 \mathrm{M}, 0.0125 \mathrm{~mol} \%)$ was introduced into a reaction vial and the solvent in the stock solution was evaporated under vacuum. Then a mixture of aniline $(5 \mathrm{mmol})$ and phenylacetylene $(6 \mathrm{mmol})$ containing $\mathrm{H}_{3} \mathrm{PW}_{12} \mathrm{O}_{4}(5.0 \mathrm{mg}, 0.03$ mol\%) and $\mathrm{KCTf}_{3}(6.0 \mathrm{mg}, 0.26 \mathrm{~mol} \%)$ was added to the vial. The stock solution of $\mathbf{L 1 -}$ $\mathrm{AuCl}$ in $\mathrm{CDCl}_{3}(12.5 \mu \mathrm{L}, 0.01 \mathrm{M}, 0.0025 \mathrm{~mol} \%)$ was added to the reaction mixture and the reaction was heated at $50{ }^{\circ} \mathrm{C}$. An aliquot (ca. $3 \mu \mathrm{L}$ ) from reaction mixture was dissolved in $\mathrm{CDCl}_{3}(0.5 \mathrm{~mL})$ and was analyzed by ${ }^{1} \mathrm{H}-\mathrm{NMR}$ to monitor the progress of the reaction. 


\section{General procedure for larger scale (10 mmol) synthesis of imine (5-2)}

To a mixture of aniline $(10 \mathrm{mmol})$ and phenylacetylene $(12 \mathrm{mmol}), \mathrm{H}_{3} \mathrm{PW}_{12} \mathrm{O}_{4}(10 \mathrm{mg}$, $0.03 \mathrm{~mol} \%)$ and $\mathrm{KCTf}_{3}(12 \mathrm{mg}, 0.26 \mathrm{~mol} \%)$ were added to the vial. The stock solution of L1-AuCl in $\mathrm{CDCl}_{3}(25 \mu \mathrm{L}, 0.01 \mathrm{M}, 0.0025 \mathrm{~mol} \%)$ was added to the reaction mixture and the reaction was heated at $50{ }^{\circ} \mathrm{C}$. An aliquot (ca. $3 \mu \mathrm{L}$ ) from the reaction mixture was dissolved in $\mathrm{CDCl}_{3}(0.5 \mathrm{~mL})$ and was analyzed by ${ }^{1} \mathrm{H}-\mathrm{NMR}$ to monitor the progress of the reaction.

\section{General procedure for synthesis of 5-5 via addition of benzotriazole 5-4 to alkyne.}

AgOTf stock solution $(100 \mu \mathrm{L}, 0.01 \mathrm{M})$ was introduced into a reaction vial and the solvent in the stock solution was evaporated under vacuum. Then 1-octyne (118 $\mu \mathrm{L}, 0.8$ mmol), benzotriazole 5-4 (27.02 mg, $0.2 \mathrm{mmol})$, and deuterated chloroform $(0.5 \mathrm{~mL})$ were added followed by the addition of stock solution of L1-AuCl $(20 \mu \mathrm{L} 0.01 \mathrm{M}, 0.1$ mol\%). The reaction was sonicated for a minute and stirred at room temperature; the progress of the reaction was monitored by NMR.

\section{General procedure for the cyclization of homopropargylic diols (5-6)}

To a solution of homopropargylic diol 5-6 $(22.64 \mu \mathrm{L}, 0.1 \mathrm{mmol})$ in $\mathrm{CDCl}_{3}(0.48 \mathrm{~mL})$ inside a NMR tube, $\mathrm{KCTf}_{3}(1.0 \mathrm{mg}, 2 \mathrm{~mol} \%)$ was added. The NMR tube was sonicated for 1-2 minute and the stock solution of $\mathrm{AgSbF}_{6}(0.01 \mathrm{M}, 0.01 \mathrm{~mol} \%)$ was added and the solution was sonicated, followed by the addition of stock solution of L1-AuCl $(2 \mu \mathrm{L}$, $0.001 \mathrm{M}, 0.002 \mathrm{~mol} \%)$. The resulting mixture was further sonicated for $15-20$ minutes at rt and the progress of reaction was monitored by ${ }^{1} \mathrm{H}$ NMR. 


\section{General procedure for the intramolecular cyclization of 4-pentynoic acid (5-8)}

To a solution of 4-pentynoic acid $\mathbf{5 - 8}(19.62 \mathrm{mg}, 0.2 \mathrm{mmol})$ in $\mathrm{CDCl}_{3}(0.48 \mathrm{~mL}), \mathrm{KCTf}_{3}$ (1.0 mg, $2 \mathrm{~mol} \%$ ) was added. The NMR tube was sonicated for 1-2 minute and the stock solution of AgOTf (0.01 M, $0.06 \mathrm{~mol} \%)$ was added and the solution was sonicated; this was followed by the addition of stock solution of L1-AuCl (2 $\mu \mathrm{L}, 0.01 \mathrm{M}, 0.01 \mathrm{~mol} \%)$. The solution was further sonicated for 15-20 minutes at $\mathrm{rt}$ and the progress of reaction was monitored by ${ }^{1} \mathrm{H}$ NMR. (Note: commercially available reactant contained hydrolyzed product as impurity).

\section{General procedure for the Conia-ene reaction of $\beta$-ketoester (5-10)}

To a solution of $\beta$-ketoester 5-10 $(9.81 \mu \mathrm{L}, 0.05 \mathrm{mmol})$ in $\mathrm{CDCl}_{3}(0.48 \mathrm{~mL})$, and stock solution of AgOTf (0.01 mol\%), HOTf $(0.01 \mathrm{~mol} \%)$ was added and the solution was sonicated, followed by the addition of stock solution of L1-AuCl $(2 \mu \mathrm{L}, 0.001 \mathrm{M}, 0.004$ mol\%). The solution was further sonicated for 15-20 minutes at $\mathrm{rt}$ and the progress of reaction was monitored by ${ }^{1} \mathrm{H}$ NMR.

\section{General procedure for the Nakamura reaction of 1,3-diketone (5-12)}

To a vial containing acetyl acetone 5-12 (103.2 $\mu \mathrm{L}, 1.0 \mathrm{mmol})$, phenyl acetylene (219.6 $\mu \mathrm{L}, 2.0 \mathrm{mmol}), \mathrm{Ga}(\mathrm{OTf})_{3}(26 \mathrm{mg}, 5 \mathrm{~mol} \%)$, L1-AuCl (50 $\left.\mu \mathrm{L}(0.01 \mathrm{M}), 0.05 \mathrm{~mol} \%\right)$ was added and the reaction was heated to $45^{\circ} \mathrm{C}$ and the progress of the reaction was monitored by ${ }^{1} \mathrm{H}$ NMR.

\section{General procedure for the cyclization of propiolate (5-14)}

To a solution of propiolate 5-14 $(12.8 \mu \mathrm{L}, 0.05 \mathrm{mmol})$ in $\mathrm{CDCl}_{3}(0.48 \mathrm{~mL})$, a stock solution of $\mathrm{AgSbF}_{6}(0.1 \mathrm{~mol} \%)$ was added and the solution was sonicated; the addition of 
stock solution of $\mathbf{L 1 - A u C l}(10 \mu \mathrm{L}, 0.001 \mathrm{M}, 0.02 \mathrm{~mol} \%)$ followed. The resulting mixture was sonicated for 15 minutes, heated to $50{ }^{\circ} \mathrm{C}$ and the progress of the reaction was monitored by ${ }^{1} \mathrm{H}$ NMR.

\section{General procedure for the hydroarylation of alkynes (5-16)}

A stock solution of $\operatorname{AgSbF}_{6}(0.25 \mathrm{~mol} \%)$ was added to a NMR tube and the solvent was evaporated under vacuum. $\mathrm{CDCl}_{3}(0.48 \mathrm{~mL})$ was added into the tube followed by the addition of 5-16 $(23.48 \mu \mathrm{L}, 0.2 \mathrm{mmol})$. Finally, the stock solution of L1-AuCl $(10 \mu \mathrm{L}$, $0.01 \mathrm{M}, 0.05 \mathrm{~mol} \%$ ) was added. The solution was sonicated for 10 minutes at $\mathrm{rt}$ and the progress of the reaction was monitored by ${ }^{1} \mathrm{H}$ NMR.

\section{General procedure for the enyn cycloisomerization of 1,6-enyne (5-18)}

To a solution of 1,6-enyne 5-18 $(42.04 \mu \mathrm{L}, 0.2 \mathrm{mmol})$ in $\mathrm{CDCl}_{3}(0.48 \mathrm{~mL})$, a stock solution of $\mathrm{AgSbF}_{6}$ (0.1 mol\%) was added followed by the addition of stock solution of L1-AuCl (4.0 $\mu \mathrm{L}, 0.01 \mathrm{M}, 0.02 \mathrm{~mol} \%)$. The solution was sonicated for 15-20 minutes at $\mathrm{rt}$ and the progress of the reaction was monitored by ${ }^{1} \mathrm{H}$ NMR.

\section{General procedure for the cycloisomerization of allenone (5-20)}

To a solution of allenone 5-20 $(23.4 \mathrm{mg}, 0.1 \mathrm{mmol})$ in $\mathrm{CDCl}_{3}(0.48 \mathrm{~mL}), \mathrm{KCTf}_{3}(1.0 \mathrm{mg}$, 2 mol\%) was added. The NMR tube was sonicated for 1-2 minute before a stock solution of $\operatorname{AgOTf}(0.05 \mathrm{~mol} \%)$ was added, the solution was sonicated again; this was followed by the addition of stock solution of $\mathbf{L 1 - A u C l}(1.0 \mu \mathrm{L}, 0.01 \mathrm{M}, 0.01 \mathrm{~mol} \%)$. The solution was then sonicated for 15-20 minutes at $\mathrm{rt}$ and the progress of reaction was monitored by ${ }^{1} \mathrm{H}$ NMR. 


\section{Monitoring of reactions using in situ NMR spectroscopy}

When ${ }^{1} \mathrm{H}$ NMR was used to monitor the progress of a reaction, a solution of tetramethylsilane in $\mathrm{CDCl}_{3}$ (sealed in a capillary tube) was used as external standard for NMR integration. In some cases, 1,3,5-tri-tert-butylbenzene (internal standard) was used. The reactions were monitored with ${ }^{1} \mathrm{H}$ NMR (single pulse or 1 scan for fast reactions, 8 scans for slow reactions). Some NMR measurements were conducted using a NMR experiment array (a series of spectra measured at predetermined time intervals over a period of time by adjusting the pre-acquisition delay). NMR experiment array gives better precision for both concentration (via integration) and reaction time, because each measurement is conducted at almost identical shimming and temperature conditions.

\section{Spectroscopic data of ligand L1 and L1AuCl:}

L1 : white solid; ${ }^{1} \mathrm{H}$ NMR $\left(\mathrm{CDCl}_{3}, 400 \mathrm{MHz}\right) \delta$ 0.60-0.85 $(6 \mathrm{H}, \mathrm{m}), 0.98-1.0(6 \mathrm{H}, \mathrm{d}, J=$ $6.0 \mathrm{~Hz}), 1.02-1.03(6 \mathrm{H}, \mathrm{d}, J=6.0 \mathrm{~Hz}), 1.13-1.15(6 \mathrm{H}, \mathrm{d}, J=6.0 \mathrm{~Hz}), 1.19-1.21(6 \mathrm{H}, \mathrm{d}, J$ $=6.0 \mathrm{~Hz}), 1.28-1.48(5 \mathrm{H}, \mathrm{m}), 4.24-4.31(2 \mathrm{H}, \mathrm{m}), 4.40-4.49(2 \mathrm{H}, \mathrm{m}), 6.39-6.41(2 \mathrm{H}, \mathrm{d}, J=$ $8.8 \mathrm{~Hz}), 6.47-6.49(2 \mathrm{H}, \mathrm{d}, J=8.0 \mathrm{~Hz}), 7.05-7.14(6 \mathrm{H}, \mathrm{m}), 7.19-7.23(2 \mathrm{H}, \mathrm{m}), 7.41-7.43$ $(2 \mathrm{H}, \mathrm{m}) ;{ }^{13} \mathrm{C} \mathrm{NMR}\left(\mathrm{CDCl}_{3}, 101 \mathrm{MHz}\right) \delta$ 21.8, 22.0, 22.4, 26.3, 27.7 (d, $\left.J=9.6 \mathrm{~Hz}\right), 29.4$ (d, $J=9.5 \mathrm{~Hz}), 35.1(\mathrm{~d}, J=19.7 \mathrm{~Hz}), 69.2,69.7,104.4,105.0,122.2,124.9,126.2,127.7$, 131.4, 135.5, $139.5(\mathrm{~d}, J=23.5 \mathrm{~Hz}), 140.8(\mathrm{~d}, J=25.1 \mathrm{~Hz}), 156.2,156.4 ;{ }^{31} \mathrm{P}$ NMR $\left(\mathrm{CDCl}_{3}, 161 \mathrm{MHz}\right) \delta$ 5.43. HRMS (ESI) Calcd. for $\mathrm{C}_{42} \mathrm{H}_{54} \mathrm{O}_{4} \mathrm{P}(\mathrm{M}+\mathrm{H})^{+}$653.3760, found 653.3755.

L1-AuCl: White solid; ${ }^{1} \mathrm{H}$ NMR $\left(\mathrm{CDCl}_{3}, 400 \mathrm{MHz}\right) \delta$ 0.66-0.76 (m, 2H), 0.82-0.90 (m, 1H), 0.94-1.04 (m, 2H), 1.05-1.07 (d, $J=6 \mathrm{~Hz}, 6 \mathrm{H}), 1.12-1.13(\mathrm{~d}, J=6 \mathrm{~Hz}, 6 \mathrm{H}), 1.16-$ $1.17(\mathrm{~d}, J=6.4 \mathrm{~Hz}, 6 \mathrm{H}), 1.19-1.21(\mathrm{~d}, J=6 \mathrm{~Hz}, 6 \mathrm{H}), 1.36-1.45(\mathrm{~m}, 3 \mathrm{H}), 1.60-1.66(\mathrm{~m}$, 
2H), 1.82-1.91 (m, 1H), 4.41-4.53 (m, 4H), 6.56-6.58 (d, J = 8.4 Hz, 4H), 6.99-7.03 (m, $2 \mathrm{H}), 7.14-7.18(\mathrm{~m}, 2 \mathrm{H}), 7.29-7.34(\mathrm{~m}, 4 \mathrm{H}), 7.51-7.56(\mathrm{~m}, 2 \mathrm{H}) ;{ }^{13} \mathrm{C} \mathrm{NMR}\left(\mathrm{CDCl}_{3}, 101\right.$ MHz) $\delta 21.9(\mathrm{~d}, J=4.1 \mathrm{~Hz}), 22.5(\mathrm{~d}, J=15.1 \mathrm{~Hz}), 25.7,26.9(\mathrm{~d}, J=15.2 \mathrm{~Hz}), 31.2,36.5$ $(\mathrm{d}, J=33.4 \mathrm{~Hz}), 69.9,70.4,105.8,106.4,121.0,125.8(\mathrm{~d}, J=10.1 \mathrm{~Hz}), 129.3,129.4$, 131.6, 132.1, 132.6 (d, $J=8.6 \mathrm{~Hz}), 136.3$ (d, $J=10.7 \mathrm{~Hz}), 140.7$ (d, $J=11.2 \mathrm{~Hz}), 156.3$, 156.7; ${ }^{31} \mathrm{P}$ NMR $\left(\mathrm{CDCl}_{3}, 161 \mathrm{MHz}\right) \delta$ 38.19. HRMS (ESI) Calcd. for $\mathrm{C}_{42} \mathrm{H}_{53} \mathrm{AuClNaO}_{4} \mathrm{P}$ $(\mathrm{M}+\mathrm{Na})^{+}$907.2933, found 907.2926. 
${ }^{1} \mathrm{H},{ }^{13} \mathrm{C}$ and ${ }^{19} \mathrm{~F}$ spectra of ligand $\mathrm{L} 1$ and array experiment.

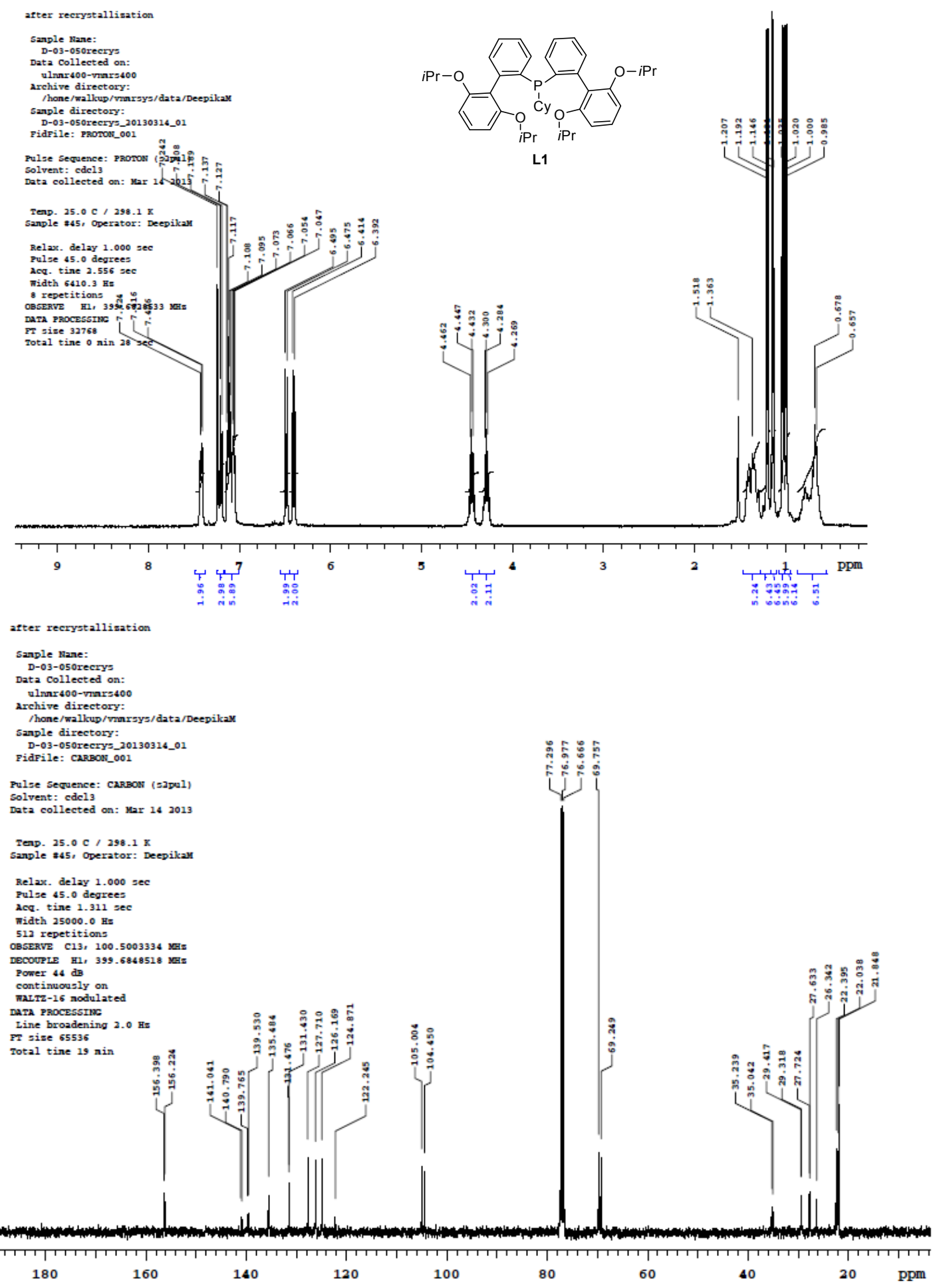


after recrystalligation

sanple nane:

D-03-050reerys
Dat 2 collected on:

vinnr400-ทnars 400

rehive directory:

/hone/mallkup/vanrsys/data/Deepikal

anple directory:

D-03-050recrys_20130316_01
FidFile: PBOSPHONOS_001

Pulse Sequence: PHOSPHOROS (s2pul)

Solvent: cde1:

on: har 142013

Tenp. $25.0 \mathrm{C} / 298.1 \mathrm{x}$

Relax. delay $1.000 \mathrm{sec}$

Pulse 45.0 degrees

acq. tine 0.996 see

width $65789.5 \mathrm{~Hz}$

OBCERVE P31, 161.7965847 NEF

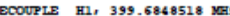

Power 44 dis

on during acquisition

ort during delay

TaATZ-16 nodulat
DATA PBOCESSTMC

Line broadening $0.5 \mathrm{~Hz}$

Total tine 2 nin $8 \mathrm{sec}$

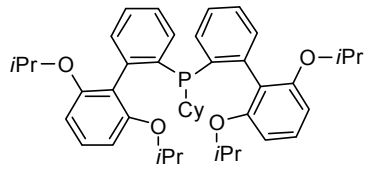

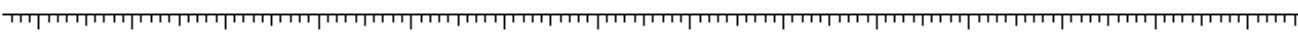

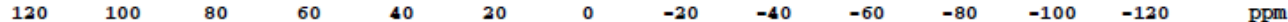




\section{Monitoring of hydroamination of 5-1}
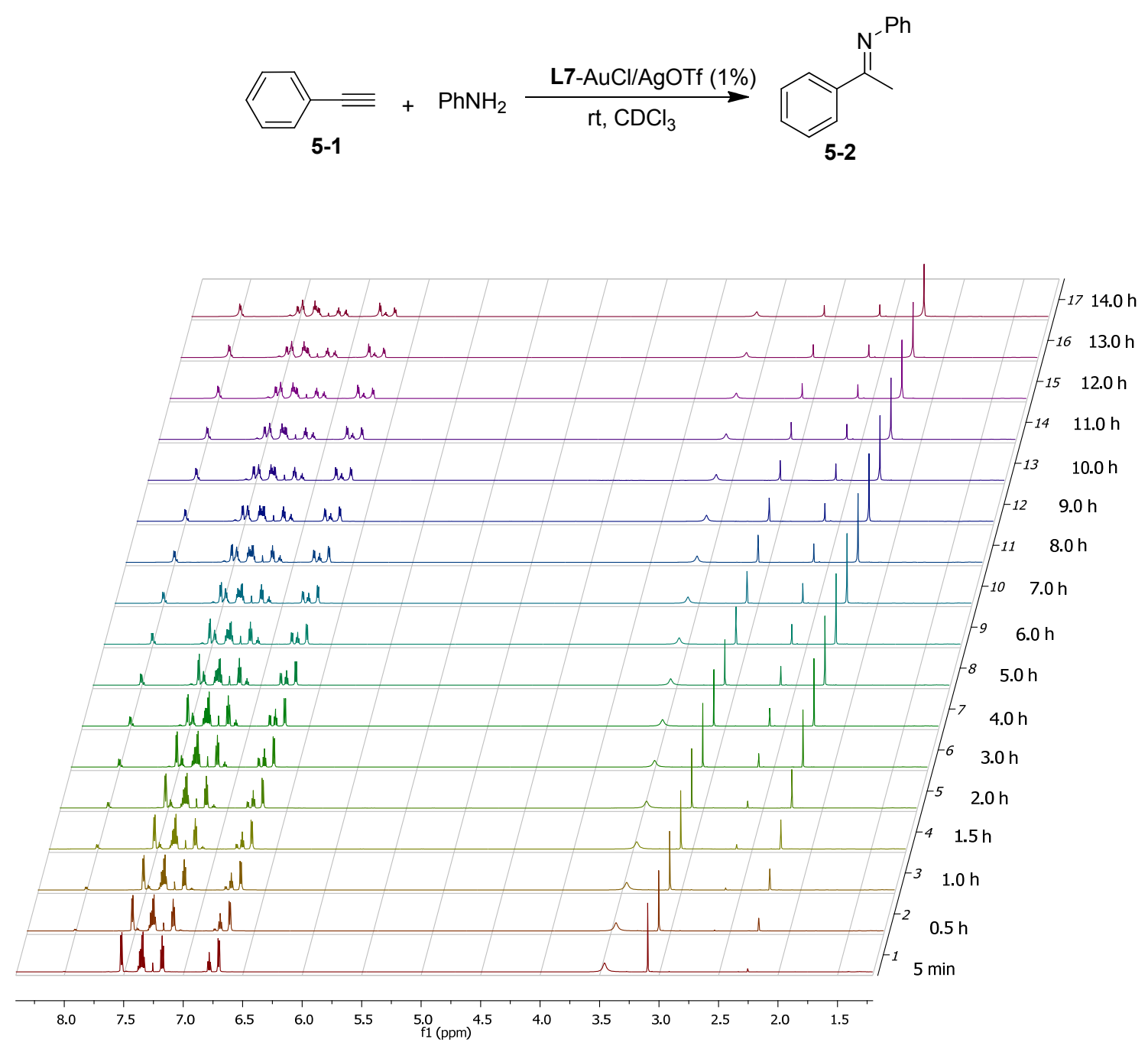


\section{CONCLUSION}

During the course of this work, we studied the gold-catalyzed intramolecular or intermolecular cyclizations involving oxonium intermediates and developed the goldcatalyzed oxygen-transfer reactions of 2-alkynyl-1,5-diketones or 2-alkynyl-5-ketoesters to furnish five-membered rings bearing a quaternary carbon tethered to a carbonyl group. Various aromatic or aliphatic groups substituted 2-alkynyl-1,5-diketones or 2-alkynyl-5ketoesters underwent cyclization in good yields under very mild conditions. The detailed mechanistic investigation of this cycloisomerization was performed on the proposed intramolecular [4+2] cycloaddition mechanism by means of ${ }^{18} \mathrm{O}$ isotopic labeling experiments and quantum chemical calculations. Both experimental and computational results corroborated the intramolecular [4+2] cycloaddition of a gold-containing furanium intermediate to a carbonyl group. Furthermore, the reactivity of alkynylenolate was investigated in the reactions of allenic ketones and vinyl ketones which lead towards the versatile synthesis of 2-alkynyl-1,5-diketones, 4-alkynyl-3-hydroxycyclohexones and 4alkynylcyclohexenones analogs in moderate to good yields. While exploring the goldcatalyzed annulations of 2-alkynyl benzaldehyde with acyclic or cyclic vinyl ethers, an approach for the synthesis of acetal-tethered dihydronaphthalene, isochromenes and bicyclo[2.2.2]octane derivatives under mild conditions was developed and these derivatives are often found in biologically active molecules and natural products. 
In order to combat the decay of the active cationic gold catalyst, which accounts for the high catalytic loading in these transformations, a modular approach has been developed for the effective catalyst design in gold catalysis to address the limitation of high catalyst loading. A highly efficient and broadly applicable cationic gold catalyst system has been successfully designed and developed and it works at extremely low catalytic loadings and relatively low temperatures. This new gold catalyst displayed high turnover numbers using mild reaction conditions, that is, room temperature or slightly elevated temperatures $\left(\leq 50^{\circ} \mathrm{C}\right)$ which would be highly desirable for the successful application of gold catalysis towards practical synthesis. This catalyst is now commercialized by Aldrich Chemicals under the name BisPhePhos XD gold(I) chloride (catalog no. L511846). In order to gain further insight into the ligand effects in gold catalysis, the quantum chemical model could be a useful tool to predict the electronics, which can guide in designing more efficient catalytic systems. In the future, we will focus our attention on exploring the application of ligand L1 in other metal catalysis such as palladium catalyzed Suzuki coupling, aminations and fluorinations. 


\section{REFERENCES}

(1) Hashmi, A. S. K.; Frost, T. M.; Bats, J. W. Highly Selective Gold-Catalyzed Arene Synthesis. J. Am. Chem. Soc. 2000, 122, 11553-11554.

(2) Hashmi, A. S. K.; Weyrauch, J. P.; Rudolph, M.; Kurpejovic, E. Gold catalysis: The benefits of N and N,O ligands. Angew. Chem., Int. Ed. 2004, 43, 6545-6547.

(3) Hashmi, A. S. K.; Rudolph, M.; Weyrauch, J. P.; Woelfle, M.; Frey, W.; Bats, J. W. Gold catalysis: Proof of arene oxides as intermediates in the phenol synthesis. Angew. Chem., Int. Ed. 2005, 44, 2798-2801.

(4) Hashmi, A. S. K.; Blanco, M. C. Gold catalysis: observation of a two-fold intermolecular hydroarylation of unactivated C-C triple bonds. Eur. J. Org. Chem. 2006, 4340-4342.

(5) Hashmi, A. S. K. Gold-catalyzed organic reactions. Chem. Rev. (Washington, DC, U. S.) 2007, 107, 3180-3211.

(6) Hashmi, A. S. K.; Buehrle, M.; Salathe, R.; Bats, J. W. Gold catalysis: Synthesis of 3-acylindenes from 2-alkynylaryl epoxides. Adv. Synth. Catal. 2008, 350, 2059-2064.

(7) Hashmi, A. S. K. Gold-catalyzed synthesis of N,O-heterocycles. Pure Appl. Chem. 2010, 82, 657-668. 
(8) Hashmi, A. S. K.; Yang, W.; Rominger, F. Gold(I)-Catalyzed Formation of Benzo[b]furans from 3-Silyloxy-1,5-enynes. Angew. Chem., Int. Ed. 2011, 50, 5762-5765.

(9) Arcadi, A. Alternative Synthetic Methods through New Developments in Catalysis by Gold. Chem. Rev. 2008, 108, 3266-3325.

(10) Gorin, D. J.; Sherry, B. D.; Toste, F. D. Ligand Effects in Homogeneous Au Catalysis. Chem. Rev. 2008, 108, 3351-3378.

(11) Jimenez-Nunez, E.; Echavarren, A. M. Gold-Catalyzed Cycloisomerizations of Enynes: A Mechanistic Perspective. Chem. Rev. (Washington, DC, U. S.) 2008, 108, $3326-3350$.

(12) Li, Z.; Brouwer, C.; He, C. Gold-Catalyzed Organic Transformations. Chem. Rev. 2008, 108, 3239-3265.

(13) Hashmi, A. S. K.; Rudolph, M. Gold catalysis in total synthesis. Chem. Soc. Rev. 2008, 37, 1766-1775.

(14) Garcia, P.; Malacria, M.; Aubert, C.; Gandon, V.; Fensterbank, L. Gold-Catalyzed Cross-Couplings: New Opportunities for C-bond-C Bond Formation. ChemCatChem 2010, 2, 493-497.

(15) Widenhoefer, R. A. Recent developments in enantioselective gold(I) catalysis. Chem.-Eur. J. 2008, 14, 5382-5391.

(16) Nolan, S. P. Catalytic gold rush. Nature 2007, 445, 496-497.

(17) Jin, T.; Yamamoto, Y. Gold-Catalyzed Intramolecular Carbocyclization of Alkynyl Ketones Leading to Highly Substituted Cyclic Enones. Org. Lett. 2007, 9, 52595262. 
(18) Rudolph, M.; Hashmi, A. S. K. Heterocycles from gold catalysis. Chem. Commun. (Cambridge, U. K.) 2011, 47, 6536-6544.

(19) Hashmi, A. S. K.; Schuster, A. M.; Gaillard, S.; Cavallo, L.; Poater, A.; Nolan, S. P. Selectivity Switch in the Synthesis of Vinylgold(I) Intermediates. Organometallics 2011, 30, 6328-6337.

(20) Hashmi, A. S. K.; Schwarz, L.; Choi, J.-H.; Frost, T. M. A new gold-catalyzed CC bond formation. Angew. Chem., Int. Ed. 2000, 39, 2285-2288.

(21) Hoffmann-Röder, A.; Krause, N. Gold(III) Chloride Catalyzed Cyclization of $\alpha$ Hydroxyallenes to 2,5-Dihydrofurans. Org. Lett. 2001, 3, 2537-2538.

(22) Asao, N.; Takahashi, K.; Lee, S.; Kasahara, T.; Yamamoto, Y. AuCl3-Catalyzed Benzannulation: Synthesis of Naphthyl Ketone Derivatives from oAlkynylbenzaldehydes with Alkynes. J. Am. Chem. Soc. 2002, 124, 12650-12651.

(23) Zhang, L.; Kozmin, S. A. Gold-Catalyzed Assembly of Heterobicyclic Systems. J. Am. Chem. Soc. 2005, 127, 6962-6963.

(24) Leyva-Pérez, A.; Corma, A. Similarities and Differences between the "Relativistic" Triad Gold, Platinum, and Mercury in Catalysis. Angew. Chem., Int. Ed. 2012, 51, 614-635.

(25) Pernpointner, M.; Hashmi, A. S. K. Fully relativistic, comparative investigation of gold and platinum alkyne complexes of relevance for the catalysis of nucleophilic additions to alkynes. J. Chem. Theory Comput. 2009, 5, 2717-2725.

(26) Gorin, D. J.; Toste, F. D. Relativistic effects in homogeneous gold catalysis. Nature 2007, 446, 395-403. 
(27) Jin, T.; Yamamoto, Y. Gold-Catalyzed Synthesis of Polycyclic Enones from Carbon Tethered 1,3-Enynyl Carbonyls via Tandem Heteroenyne Metathesis and Nazarov Reaction. Org. Lett. 2008, 10, 3137-3139.

(28) Krafft, M. E.; Vidhani, D. V.; Cran, J. W.; Manoharan, M. Solvent controlled mechanistic dichotomy in a Au(iii)-catalyzed, heterocyclization triggered, Nazarov reaction. Chem. Commun. 2011, 47, 6707-6709.

(29) Rodríguez, A.; Moran, W. J. Tetrahedron Lett. 2011, 52, 2605-2607.

(30) Wang, T.; Shi, S.; Vilhelmsen, M. H.; Zhang, T.; Rudolph, M.; Rominger, F.; Hashmi, A. S. K. Chemoselectivity Control: Gold(I)-Catalyzed Synthesis of 6,7Dihydrobenzofuran-4(5H)-ones and Benzofurans from 1-(Alkynyl)-7oxabicyclo[4.1.0]heptan-2-ones. Chem. Eur. J. 2013, 19, 12512-12516.

(31) Hashmi, A. S. K.; Sinha, P. Gold Catalysis: Mild Conditions for the Transformation of Alkynyl Epoxides to Furans. Adv. Synth. Catal. 2004, 346, 432-438.

$$
\text { Shu, X.-Z.; Liu, X.-Y.; Xiao, H.-Q.; Ji, K.-G.; Guo, L.-N.; Qi, C.-Z.; Liang, Y.-M. }
$$

Gold-Catalyzed Tandem Cycloisomerization of Alkynyloxiranes with Nucleophiles: An Efficient Approach to 2,5-Disubstituted Furans. Adv. Synth. Catal. 2007, 349, 2493-2498.

(33) Ji, K.-G.; Shu, X.-Z.; Chen, J.; Zhao, S.-C.; Zheng, Z.-J.; Liu, X.-Y.; Liang, Y.-M. Gold-catalyzed tandem cyclization/Friedel-Crafts type reactions toward furan derivatives. Org. Biomol. Chem. 2009, 7, 2501-2505.

(34) Renault, J.; Qian, Z.; Uriac, P.; Gouault, N. Electrophilic carbon transfer in gold catalysis: synthesis of substituted chromones. Tetrahedron Lett. 2011, 52, 2476-2479. 
(35) Malhotra, D.; Liu, L.-P.; Hammond, G. B. Tandem Michael Addition/Aldol Reaction of Allenic Ketones with Alkyl Vinyl Ketones: Versatile Synthesis of 2-Alkynyl 1,5-Diketones, 4-Alkynyl-3-hydroxycyclohexanones and 4-Alkynylcyclohexenones. Eur. J. Org. Chem. 2010, 6855-6862.

(36) Asao, N.; Aikawa, H.; Yamamoto, Y. AuBr3-Catalyzed [4 + 2] Benzannulation between an Enynal Unit and Enol. J. Am. Chem. Soc. 2004, 126, 7458-7459.

(37) Shen, Q.; Shekhar, S.; Stambuli, J. P.; Hartwig, J. F. Highly Reactive, General, and Long-Lived Catalysts for Coupling Heteroaryl and Aryl Chlorides with Primary Nitrogen Nucleophiles. Angew. Chem., Int. Ed. 2005, 44, 1371-1375.

(38) Gorin, D. J.; Sherry, B. D.; Toste, F. D. Ligand Effects in Homogeneous Au Catalysis. Chem. Rev. (Washington, DC, U. S.) 2008, 108, 3351-3378.

(39) Wang, Z. J.; Benitez, D.; Tkatchouk, E.; Goddard III, W. A.; Toste, F. D. Mechanistic Study of Gold(I)-Catalyzed Intermolecular Hydroamination of Allenes. J. Am. Chem. Soc. 2010, 132, 13064-13071.

(40) Markham, J. P.; Staben, S. T.; Toste, F. D. Gold(I)-Catalyzed Ring Expansion of Cyclopropanols and Cyclobutanols. J. Am. Chem. Soc. 2005, 127, 9708-9709.

(41) Wang, W.; Hammond, G. B.; Xu, B. Ligand Effects and Ligand Design in Homogeneous Gold(I) Catalysis. J. Am. Chem. Soc. 2012, 134, 5697-5705.

(42) Marion, N.; Ramón, R. S.; Nolan, S. P. [(NHC)AuI]-Catalyzed Acid-Free Alkyne Hydration at Part-per-Million Catalyst Loadings. J. Am. Chem. Soc. 2008, 131, 448-449. 
(43) Lavallo, V.; Wright, J. H.; Tham, F. S.; Quinlivan, S. Perhalogenated Carbacloso-dodecaborate Anions as Ligand Substituents: Applications in Gold Catalysis. Angew. Chem., Int. Ed. 2013, 52, 3172-3176.

(44) Oliver-Meseguer, J.; Cabrero-Antonino, J. R.; Domínguez, I.; Leyva-Pérez, A.; Corma, A. Science 2012, 338, 1452-1455.

(45) Blanco Jaimes, M. C.; Böhling, C. R. N.; Serrano-Becerra, J. M.; Hashmi, A. S. K. Highly Active Mononuclear NAC-Gold(I) Catalysts. Angew. Chem., Int. Ed. 2013, 52, 7963-7966.

(46) Liu, L.-P.; Xu, B.; Mashuta, M. S.; Hammond, G. B. Synthesis and Structural Characterization of Stable Organogold(I) Compounds. Evidence for the Mechanism of Gold-Catalyzed Cyclizations. J. Am. Chem. Soc. 2008, 130, 17642-17643.

(47) Sibi, M. P.; Manyem, S. Enantioselective Conjugate Additions. Tetrahedron 2000, $56,8033-8061$.

(48) Feringa, B. L. Phosphoramidites: Marvellous Ligands in Catalytic Asymmetric Conjugate Addition. Acc. Chem. Res. 2000, 33, 346-353.

(49) Woodward, S. Decoding the "black box" reactivity that is organocuprate conjugate addition chemistry. Chem. Soc. Rev. 2000, 29, 393-401.

(50) Alexakis, A.; Benhaim, C. Enantioselective copper-catalyzed conjugate addition. Eur. J. Org. Chem. 2002, 3221-3236.

(51) Lopez, F.; Minnaard, A. J.; Feringa, B. L. Catalytic enantioselective conjugate addition with Grignard reagents. Acc. Chem. Res. 2007, 40, 179-188. 
(52) Sulzer-Mosse, S.; Alexakis, A. Chiral amines as organocatalysts for asymmetric conjugate addition to nitroolefins and vinyl sulfones via enamine activation. Chem. Commun. (Cambridge, U. K.) 2007, 3123-3135.

(53) Toure, B. B.; Hall, D. G. Natural Product Synthesis Using Multicomponent Reaction Strategies. Chem. Rev. (Washington, DC, U. S.) 2009, 109, 4439-4486.

(54) Trost, B. M.; Brindle, C. S. The direct catalytic asymmetric aldol reaction. Chem. Soc. Rev. 2010, 39, 1600-1632.

(55) Brodmann, T.; Lorenz, M.; Schaeckel, R.; Simsek, S.; Kalesse, M. Highly stereoselective aldol reactions in the total syntheses of complex natural products. Synlett 2009, 174-192.

(56) Mlynarski, J.; Paradowska, J. Catalytic asymmetric aldol reactions in aqueous media. Chem. Soc. Rev. 2008, 37, 1502-1511.

(57) Ramazani, A.; Kazemizadeh, A. R.; Ahmadi, E.; Noshiranzadeh, N.; Souldozi, A. Synthesis and reactions of stabilized phosphorus ylides. Curr. Org. Chem. 2008, 12, 5982.

(58) Pietrusiewicz, K. M.; Zablocka, M. Preparation of Scalemic P-Chiral Phosphines and Their Derivatives. Chem. Rev. (Washington, D. C.) 1994, 94, 1375-411.

(59) Cobridge, D. E. C. Phosphorus: An Outline of its Chemistry, Biochemistry and Uses; Elsevier: Amsterdam, 1995.

(60) Engel, R. Synthesis of Carbon-Phosphorus Bonds 1988. 
(61) Maryanoff, B. E.; Reitz, A. B. The Wittig olefination reaction and modifications involving phosphoryl-stabilized carbanions. Stereochemistry, mechanism, and selected synthetic aspects. Chem. Rev. 1989, 89, 863-927.

(62) Cadogan, J. I. G. Organophosphorus Reagents in Organic Synthesis, Academic Press: New York, 1979.

(63) Harding, C. E. H., M. Tetrahedron Lett. 1971, 12, 1253.

(64) Balf, R. J.; Rao, B.; Weiler, L. Transannular cyclizations of 6-substituted cyclodecynes. Can. J. Chem. 1971, 49, 3135-3142.

(65) Hanack, M.; Harding, C. E.; Derocque, J. L. Transannular rearrangements of cyclodecynyl derivatives. Chem. Ber. 1972, 105, 421-33.

(66) Lange, G. L.; Hall, T.-W. Synthesis and reactions of 5-cyclononynone. J. Org. Chem. 1974, 39, 3819-21.

(67) Harding, C. E.; Stanford, G. R., Jr. Mechanism of the transannular cyclization of 5-cyclodecynone. J. Org. Chem. 1989, 54, 3054-6.

(68) Harding, C. E.; King, S. L. Mechanism of the intramolecular cyclization of acetylenic ketones. J. Org. Chem. 1992, 57, 883-6.

(69) Sisko, J.; Balog, A.; Curran, D. P. Annulative ring expansions. Direct conversion of $\omega$-alkynyl acetals to polycyclic unsaturated ketones. J. Org. Chem. 1992, 57, 4341-2.

(70) Grunwell, J. R.; Wempe, M. F.; Mitchell, J.; Grunwell, J. R. The transannular rearrangement of 5-cyclodecynone. Tetrahedron Lett. 1993, 34, 7163-6. 
(71) Balog, A.; Curran, D. P. Ring-Enlarging Annulations. A One-Step Conversion of Cyclic Silyl Acyloins and w-Alkynyl Acetals to Polycyclic Enediones. J. Org. Chem. 1995, 60, 337-44.

(72) Balog, A.; Geib, S. V.; Curran, D. P. Additive and Medium Effects on Lewis Acid-Promoted Cationic $\pi$-Cyclizations of Alkenyl- and Alkynylcyclopentane-1,3-diones. J. Org. Chem. 1995, 60, 345-52.

(73) Wempe, M. F. G., J. R. J. Org. Chem. 1995, 60, 2714.

(74) Wempe, M. F.; Grunwell, J. R. 13C Scrambling of $\left[5-{ }^{13} \mathrm{C}\right] 5$-cyclodecynone. Tetrahedron Lett. 2000, 41, 6709-6713.

(75) Viswanathan, G. S.; Li, C.-J. A highly stereoselective, novel coupling reaction between alkynes and aldehydes. Tetrahedron Lett. 2002, 43, 1613-1615.

(76) Curini, M.; Epifano, F.; Maltese, F.; Rosati, O. Ytterbium triflate promoted coupling reaction between aryl alkynes and aldehydes. Synlett 2003, 552-554.

(77) Rhee, J. U.; Krische, M. J. Alkynes as Synthetic Equivalents to Stabilized Wittig Reagents: Intra- and Intermolecular Carbonyl Olefinations Catalyzed by $\mathrm{Ag}(\mathrm{I}), \mathrm{BF}_{3}$, and HBF4. Org. Lett. 2005, 7, 2493-2495.

(78) Saito, A.; Umakoshi, M.; Yagyu, N.; Hanzawa, Y. Novel One-Pot Approach to Synthesis of Indanones through $\mathrm{Sb}(\mathrm{V})$-Catalyzed Reaction of Phenylalkynes with Aldehydes. Org. Lett. 2008, 10, 1783-1785.

(79) Gonzalez-Rodriguez, C.; Escalante, L.; Varela, J. A.; Castedo, L.; Saa, C. Bronsted Acid-Promoted Intramolecular Carbocyclization of Alkynals Leading to Cyclic Enones. Org. Lett. 2009, 11, 1531-1533. 
(80) Kurtz, K. C. M.; Hsung, R. P.; Zhang, Y. A Ring-Closing Yne-Carbonyl Metathesis of Ynamides. Org. Lett. 2006, 8, 231-234.

(81) Yamamoto, Y.; Gridnev, I. D.; Patil, N. T.; Jin, T. Alkyne activation with Bronsted acids, iodine, or gold complexes, and its fate leading to synthetic application. Chem. Commun. (Cambridge, U. K.) 2009, 5075-5087.

(82) $\mathrm{Xu}, \mathrm{B}$.; Hammond, G. B. From vinylogation to alkynylogation: extending the reactivity of enolates. Synlett 2010, 1442-1454.

(83) Xu, B.; Hammond, G. B. Thermodynamically favored aldol reaction of propargyl or allenyl esters: regioselective synthesis of carbinol allenoates. Angew. Chem., Int. Ed. 2008, 47, 689-692.

(84) Yang, H.; Xu, B.; Hammond, G. B. Highly Regioselective Fluorination and Iodination of Alkynyl Enolates. Org. Lett. 2008, 10, 5589-5591.

(85) Wang, W.; Xu, B.; Hammond, G. B. Synthesis of Functionalized $\alpha, \alpha-$ Disubstituted $\beta$-Alkynyl Esters from Allenoates through an Alkynylenolate Intermediate. Org. Lett. 2008, 10, 3713-3716.

(86) Liu, L.-P.; Xu, B.; Hammond, G. B. Michael Addition of Allenoates to ElectronDeficient Olefins: Facile Synthesis of 2-Alkynyl-Substituted Glutaric Acid Derivatives. Org. Lett. 2008, 10, 3887-3890.

(87) Liu, L.-P.; Hammond, G. B. Highly Efficient and Tunable Synthesis of Dioxabicyclo[4.2.1] Ketals and Tetrahydropyrans via Gold-Catalyzed Cycloisomerization of 2-Alkynyl-1,5-diols. Org. Lett. 2009, 11, 5090-5092. 
(88) Patil, N. T.; Yamamoto, Y. Coinage Metal-Assisted Synthesis of Heterocycles. Chem. Rev. (Washington, DC, U. S.) 2008, 108, 3395-3442.

(89) Asao, N. Gold- and copper-catalyzed [4+2] benzannulations between enynal or

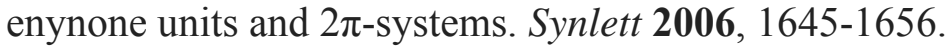

(90) Kusama, H.; Iwasawa, N. Cycloaddition reactions of transition metal-containing benzopyrylium and related zwitterionic intermediates. Chem. Lett. 2006, 35, 1082-1087.

(91) Kotha, S.; Misra, S.; Halder, S. Benzannulation. Tetrahedron 2008, 64, 1077510790.

(92) Das, A.; Abu Sohel, S. M.; Liu, R.-S. Carbo- and heterocyclization of oxygenand nitrogen-containing electrophiles by platinum, gold, silver and copper species. Org. Biomol. Chem. 2010, 8, 960-979.

(93) Asao, N.; Nogami, T.; Lee, S.; Yamamoto, Y. Lewis Acid-Catalyzed Benzannulation via Unprecedented [4+2] Cycloaddition of o-Alkynyl(oxo)benzenes and Enynals with Alkynes. J. Am. Chem. Soc. 2003, 125, 10921-10925.

(94) Asao, N.; Kasahara, T.; Yamamoto, Y. Functionalized 1,2-dihydronaphthalenes from the $\mathrm{Cu}(\mathrm{OTf}) 2$-catalyzed [4+2] cycloaddition of o-alkynyl(oxo)benzenes with alkenes. Angew. Chem., Int. Ed. 2003, 42, 3504-3506.

(95) Asao, N.; Nogami, T.; Takahashi, K.; Yamamoto, Y. Pd(II) Acts Simultaneously as a Lewis Acid and as a Transition-Metal Catalyst: Synthesis of Cyclic Alkenyl Ethers from Acetylenic Aldehydes. J. Am. Chem. Soc. 2002, 124, 764-765.

(96) Mondal, S.; Nogami, T.; Asao, N.; Yamamoto, Y. Synthesis of Novel Antitumor Agent 1-Methoxy-5,10-dioxo-5,10-dihydro-1H-benzo[g]isochromene Carboxylic Acid 
(3-Dimethylylaminopropyl)amide with a Dual Role Pd(II) Catalyst. J. Org. Chem. 2003, 68, 9496-9498.

(97) Patil, N. T.; Yamamoto, Y. Synthesis of Cyclic Alkenyl Ethers via Intramolecular Cyclization of O-Alkynylbenzaldehydes. Importance of Combination between CuI Catalyst and DMF. J. Org. Chem. 2004, 69, 5139-5142.

(98) Nakamura, H.; Ohtaka, M.; Yamamoto, Y. Tandem nucleophilic allylationalkoxyallylation of alkynylaldehydes via amphiphilic bis- $\pi$-allylpalladium complexes. Tetrahedron Lett. 2002, 43, 7631-7633.

(99) Iwasawa, N.; Shido, M.; Maeyama, K.; Kusama, H. Novel Synthesis of Pentacarbonylbenzopyranylidenetungsten(0) Complexes and Their Diels-Alder Reaction with Electron-Rich Alkenes. J. Am. Chem. Soc. 2000, 122, 10226-10227.

(100) Iwasawa, N.; Shido, M.; Kusama, H. Generation and reaction of metal-containing carbonyl ylides: tandem [3+2]-cycloaddition-carbene insertion leading to novel polycyclic compounds. J. Am. Chem. Soc. 2001, 123, 5814-5815.

(101) Kusama, H.; Takaya, J.; Iwasawa, N. A Facile Method for the Synthesis of Polycyclic Indole Derivatives: The Generation and Reaction of Tungsten-Containing Azomethine Ylides. J. Am. Chem. Soc. 2002, 124, 11592-11593.

(102) Kusama, H.; Funami, H.; Shido, M.; Hara, Y.; Takaya, J.; Iwasawa, N. Generation and Reaction of Tungsten-Containing Carbonyl Ylides: [3+2]-Cycloaddition Reaction with Electron-Rich Alkenes. J. Am. Chem. Soc. 2005, 127, 2709-2716.

(103) Kusama, H.; Funami, H.; Takaya, J.; Iwasawa, N. Platinum(II)-catalyzed reaction of 2-alkynylbenzoates or -benzothioates with vinyl ethers: A concise method for 
synthesis of 1-acyl-4-alkoxy- or 1-acyl-4-alkylsulfanylnaphthalene derivatives. Org. Lett. 2004, 6, 605-608.

(104) Kusama, H.; Ishida, K.; Funami, H.; Iwasawa, N. Platinum(II)-catalyzed reaction of $\gamma, \delta$-ynones with alkenes for the construction of 8-oxabicyclo[3.2.1]octane skeletons: generation of platinum-containing carbonyl ylides from acyclic precursors. Angew. Chem., Int. Ed. 2008, 47, 4903-4905.

(105) Takaya, J.; Udagawa, S.; Kusama, H.; Iwasawa, N. Synthesis of N-fused tricyclic indoles by a tandem [1,2] Stevens-type rearrangement/1,2-alkyl migration of metalcontaining ammonium ylides. Angew. Chem., Int. Ed. 2008, 47, 4906-4909.

(106) Barluenga, J.; Vazquez-Villa, H.; Ballesteros, A.; Gonzalez, J. M. Cyclization of Carbonyl Groups onto Alkynes upon Reaction with IPy2BF4 and Their Trapping with Nucleophiles: A Versatile Trigger for Assembling Oxygen Heterocycles. J. Am. Chem. Soc. 2003, 125, 9028-9029.

(107) Barluenga, J.; Vazquez-Villa, H.; Ballesteros, A.; Gonzalez, J. M. Regioselective Synthesis of Substituted Naphthalenes: A Novel de Novo Approach Based on a MetalFree Protocol for Stepwise Cycloaddition of o-Alkynylbenzaldehyde Derivatives with Either Alkynes or Alkenes. Org. Lett. 2003, 5, 4121-4123.

(108) Barluenga, J.; Vazquez-Villa, H.; Ballesteros, A.; Gonzalez, J. M. Synthesis of indoles upon sequential reaction of 3-alkynylpyrrole-2-carboxaldehydes with iodonium ions and alkenes. Preparation of related benzofuran and benzothiophene derivatives. Adv. Synth. Catal. 2005, 347, 526-530. 
(109) Barluenga, J.; Vazquez-Villa, H.; Merino, I.; Ballesteros, A.; Gonzalez, J. M. The reaction of o-alkynylarene and heteroarene carboxaldehyde derivatives with iodonium ions and nucleophiles: a versatile and regioselective synthesis of $1 \mathrm{H}$-isochromene, naphthalene, indole, benzofuran, and benzothiophene compounds. Chem. - Eur. J. 2006, $12,5790-5805$.

(110) Oh, C. H.; Lee, J. H.; Lee, S. J.; Kim, J. I.; Hong, C. S. Intramolecular Huisgentype cyclization of platinum-bound pyrylium ions with alkenes and subsequent insertion into a benzylic C-H bond. Angew. Chem., Int. Ed. 2008, 47, 7505-7507.

(111) Oh, C. H.; Lee, J. H.; Lee, S. M.; Yi, H. J.; Hong, C. S. Divergent insertion reactions of Pt-carbenes generated from [3+2] cyclization of platinum-bound pyrylliums. Chem. - Eur. J. 2009, 15, 71-74.

(112) Kim, N.; Kim, Y.; Park, W.; Sung, D.; Gupta, A. K.; Oh, C. H. Gold-Catalyzed Cycloisomerization of o-Alkynylbenzaldehydes with a Pendant Unsaturated Bond: [3 + 2] Cycloaddition of Gold-Bound 1,3-Dipolar Species with Dipolarophiles. Org. Lett. 2005, 7, 5289-5291.

(113) Bhunia, S.; Wang, K.-C.; Liu, R.-S. Pt ${ }^{\mathrm{II}}$-Catalyzed synthesis of 9oxabicyclo[3.3.1]nona-2,6-dienes from 2-alkynyl-1-carbonylbenzenes and allylsilanes by an allylation/annulation cascade. Angew. Chem., Int. Ed. 2008, 47, 5063-5066.

(114) Hsu, Y.-C.; Ting, C.-M.; Liu, R.-S. Stereocontrolled synthesis of complicated oxacyclic compounds via platinum-catalyzed $[4+2]$-cycloadditions and annulations of enynals with allylic alcohols. J. Am. Chem. Soc. 2009, 131, 2090-2091. 
(115) Yue, D.; Della Ca, N.; Larock, R. C. Efficient syntheses of heterocycles and carbocycles by electrophilic cyclization of acetylenic aldehydes and ketones. Org. Lett. 2004, 6, 1581-1584.

(116) Yue, D.; Della Ca, N.; Larock, R. C. Syntheses of Isochromenes and Naphthalenes by Electrophilic Cyclization of Acetylenic Arenecarboxaldehydes. J. Org. Chem. 2006, 71, 3381-3388.

(117) Waldo, J. P.; Larock, R. C. The Synthesis of Highly Substituted Isoxazoles by Electrophilic Cyclization: An Efficient Synthesis of Valdecoxib. J. Org. Chem. 2007, 72, 9643-9647.

(118) Hu, Z.-L.; Qian, W.-J.; Wang, S.; Wang, S.; Yao, Z.-J. Transformation of Reactive Isochromenylium Intermediates to Stable Salts and Their Cascade Reactions with Olefins. Org. Lett. 2009, 11, 4676-4679.

(119) Hu, Z.-L.; Qian, W.-J.; Wang, S.; Wang, S.; Yao, Z.-J. Construction of Multiring Frameworks by Metal-Free Cascade Reactions of Stable Isochromenylium Tetrafluoroborate. J. Org. Chem. 2009, 74, 8787-8793.

(120) Dyker, G.; Hildebrandt, D.; Liu, J.; Merz, K. Gold(III) chloride catalyzed domino processes with isobenzopyrylium cation intermediates. Angew. Chem., Int. Ed. 2003, 42, 4399-4402.

(121) Hildebrandt, D.; Hueggenberg, W.; Kanthak, M.; Ploeger, T.; Mueller, I. M.; Dyker, G. A platinum-catalyzed annulation reaction leading to medium-sized rings. Chem. Commun. (Cambridge, U. K.) 2006, 2260-2261. 
(122) Beeler, A. B.; Su, S.; Singleton, C. A.; Porco, J. A., Jr. Discovery of Chemical Reactions through Multidimensional Screening. J. Am. Chem. Soc. 2007, 129, 1413-1419. (123) Singh, V.; Krishna, U. M.; Vikrant; Trivedi, G. K. Cycloaddition of oxidopyrylium species in organic synthesis. Tetrahedron 2008, 64, 3405-3428.

(124) Li, Z.; Brouwer, C.; He, C. Gold-Catalyzed Organic Transformations. Chem. Rev. (Washington, DC, U. S.) 2008, 108, 3239-3265.

(125) Arcadi, A. Alternative Synthetic Methods through New Developments in Catalysis by Gold. Chem. Rev. (Washington, DC, U. S.) 2008, 108, 3266-3325.

(126) Hashmi, A. S. K. Homogeneous Gold Catalysis Beyond Assumptions and Proposals-Characterized Intermediates. Angew. Chem., Int. Ed. 2010, 49, 5232-5241.

(127) Liu, L.-P.; Hammond, G. B. Reactions of Cationic Gold(I) with Allenoates: Synthesis of Stable Organogold(I) Complexes and Mechanistic Investigations on GoldCatalyzed Cyclizations. Chem. - Asian J. 2009, 4, 1230-1236.

(128) Liu, L.-P.; Hammond, G. B. Gold-Catalyzed Cascade Annulations of 2-(Ynol)aryl Aldehydes: Facile Synthesis of Benzochromanes and Benzobicyclo[n.3.1]acetals. Org. Lett. 2010, 12, 4640-4643.

(129) Wang, W.; Xu, B.; Hammond, G. B. Efficient Synthesis of $\gamma$-Keto Esters through Neighboring Carbonyl Group-Assisted Regioselective Hydration of 3-Alkynoates. J. Org. Chem. 2009, 74, 1640-1643.

(130) Wang, W.; Jasinski, J.; Hammond, G. B.; Xu, B. Fluorine-enabled cationic gold catalysis: functionalized hydration of alkynes. Angew. Chem., Int. Ed. 2010, 49, 72477252. 
(131) Liu, L.-P.; Malhotra, D.; Paton, R. S.; Houk, K. N.; Hammond, G. B. The [4+2], not [2+2], Mechanism Occurs in the Gold-Catalyzed Intramolecular Oxygen Transfer Reaction of 2-Alkynyl-1,5-Diketones. Angew. Chem., Int. Ed. 2010, 49, 9132-9135.

(132) Northcote, P. T.; Andersen, R. J. Xestenone, a new bicyclic C-19 terpenoid from the marine sponge Xestospongia vanilla. Tetrahedron Lett. 1988, 29, 4357-60.

(133) Ota, K.; Kurokawa, T.; Kawashima, E.; Miyaoka, H. Total synthesis and absolute configuration of the marine norditerpenoid xestenone. Mar. Drugs 2009, 7, 654-671.

(134) Cheng, X.-C.; Varoglu, M.; Abrell, L.; Crews, P.; Lobkovsky, E.; Clardy, J. Chloriolins A-C, Chlorinated Sesquiterpenes Produced by Fungal Cultures Separated from a Jaspis Marine Sponge. J. Org. Chem. 1994, 59, 6344-8.

(135) Sahu, N. P.; Koike, K.; Banerjee, S.; Achari, B.; Nikaido, T. Triterpenoid saponins from Mollugo spergula. Phytochemistry 2001, 58, 1177-1182.

(136) Kitagawa, I.; Suzuki, H.; Kitazawa, K.; Yamao, N.; Yosioka, I. Saponin and sapogenol. IX. Structure of spergulagenin A, a new migrated hopane-type sapogenol isolated from Mollugo spergula. Chem. Pharm. Bull. 1975, 23, 355-64.

(137) Kitagawa, I. S., H.; Yosioka, I.; Akiyama, T.; Silverton, J. V. Tetrahedron Lett. 1974, 15, 1173.

(138) Kitagawa, I.; Yamanaka, H.; Nakanishi, T.; Yosioka, I. Saponin and sapogenol. XXII. Structure of spergulatriol, a new bisnorhopane-type genuine sapogenol of isoanhydrospergulatriol, from the root of Mollugo spergula L. Chem. Pharm. Bull. 1977, 25, 2430-6. 
(139) Singh, I. P.; Talwar, K. K.; Arora, J. K.; Chhabra, B. R.; Kalsi, P. S. A biologically active guaianolide from Saussurea lappa. Phytochemistry 1992, 31, 2529-31.

(140) Talwar, K. K.; Singh, I. P.; Kalsi, P. S. A sesquiterpenoid with plant growth regulatory activity from Saussurea lappa. Phytochemistry 1991, 31, 336-8.

(141) Rao, K. S.; Babu, G. V.; Ramnareddy, Y. V. Acylated flavone glycosides from the roots of Saussurea lappa and their antifungal activity. Molecules 2007, 12, 328-344.

(142) Jin, T.; Yang, F.; Liu, C.; Yamamoto, Y. TfOH-catalyzed intramolecular alkyneketone metathesis leading to highly substituted five-membered cyclic enones. Chem. Commun. (Cambridge, U. K.) 2009, 3533-3535.

(143) Yao, T.; Zhang, X.; Larock, R. C. AuCl3-Catalyzed synthesis of highly substituted furans from 2-(1-alkynyl)-2-alken-1-ones. J. Am. Chem. Soc. 2004, 126, 11164-11165.

(144) Liu, Y.; Liu, M.; Guo, S.; Tu, H.; Zhou, Y.; Gao, H. Gold-catalyzed highly efficient access to 3(2H)-furanones from 2-oxo-3-butynoates and related compounds. Org. Lett. 2006, 8, 3445-3448.

(145) Schwier, T.; Sromek, A. W.; Yap, D. M. L.; Chernyak, D.; Gevorgyan, V. Mechanistically diverse copper-, silver-, and gold-catalyzed acyloxy and phosphatyloxy migrations: efficient synthesis of heterocycles via cascade migration/cycloisomerization approach. J. Am. Chem. Soc. 2007, 129, 9868-9878.

(146) Zhang, J.; Schmalz, H.-G. Gold(I)-catalyzed reaction of 1-(1-alkynyl)cyclopropyl ketones with nucleophiles: a modular entry to highly substituted furans. Angew. Chem., Int. Ed. 2006, 45, 6704-6707. 
(147) Kirsch, S. F.; Binder, J. T.; Liebert, C.; Menz, H. Gold(III)- and platinum(II)catalyzed domino reaction consisting of heterocyclization and 1,2-migration: efficient synthesis of highly substituted 3(2H)-furanones. Angew. Chem., Int. Ed. 2006, 45, 58785880.

(148) Chan, C. S.; Araki, T.; Nakamura, I.; Terada, M. Gold-catalyzed consecutive [1,2] alkyl migration-oxygen transfer reaction of 2-alkynyl-1-tetralones. Tetrahedron Lett. 2009, 50, 216-218.

(149) Zhang, G.; Huang, X.; Li, G.; Zhang, L. Au-Containing All-Carbon 1,4-Dipoles: Generation and [4+2] Annulation in the Formation of Carbo-/Heterocycles. J. Am. Chem. Soc. 2008, 130, 1814-1815.

(150) Li, G.; Huang, X.; Zhang, L. Au(I)-Catalyzed Efficient Synthesis of Functionalized Bicyclo[3.2.0]heptanes. J. Am. Chem. Soc. 2008, 130, 6944-6945.

(151) Liu, F.; Yu, Y.; Zhang, J. Highly Substituted Furo[3,4-d][1,2]oxazines: GoldCatalyzed Regiospecific and Diastereoselective 1,3-Dipolar Cycloaddition of 2-(1Alkynyl)-2-alken-1-ones with Nitrones. Angew. Chem., Int. Ed. 2009, 48, 5505-5508.

(152) Li, W.; Li, Y.; Zhang, J. Gold-Catalyzed Domino Reactions Consisting of Regioand Stereoselective 1,2-Alkyl Migration. Chem. - Eur. J. 2010, 16, 6447-6450.

(153) Vederas, J. C. Structural dependence of oxygen-18 isotope shifts in carbon-13 NMR spectra. J. Am. Chem. Soc. 1980, 102, 374-6.

(154) Hashmi, A. S. K.; Schaefer, S.; Woelfe, M.; Diez Gil, C.; Fischer, P.; Laguna, A.; Blanco, M. C.; Gimeno, M. C. Gold-catalyzed benzylic C-H activation at room temperature. Angew. Chem., Int. Ed. 2007, 46, 6184-6187. 
(155) Tang, J.-M.; Liu, T.-A.; Liu, R.-S. Gold-Catalyzed Hydrative Carbocyclization of 1,5- and 1,6-Diyn-3-ones via an Oxygen Transfer Process. J. Org. Chem. 2008, 73, 84798483.

(156) Byrn, M.; Calvin, M. Oxygen-18 exchange reactions of aldehydes and ketones. $J$. Am. Chem. Soc. 1966, 88, 1916-22.

(157) Gaussian 09, Revision A.02, M. J. Frisch, G. W. Trucks, H. B. Schlegel, G. E. Scuseria, M. A. Robb, J. R. Cheeseman, G. Scalmani, V. Barone, B. Mennucci, G. A. Petersson, H. Nakatsuji, M. Caricato, X. Li, H. P. Hratchian, A. F. Izmaylov, J. Bloino, G. Zheng, J. L. Sonnenberg, M. Hada, M. Ehara, K. Toyota, R. Fukuda, J. Hasegawa, M. Ishida, T. Nakajima, Y. Honda, O. Kitao, H. Nakai, T. Vreven, J. A. Montgomery, Jr., J. E. Peralta, F. Ogliaro, M. Bearpark, J. J. Heyd, E. Brothers, K. N. Kudin, V. N. Staroverov, R. Kobayashi, J. Normand, K. Raghavachari, A. Rendell, J. C. Burant, S. S. Iyenga, J. Tomasi, M. Cossi, N. Rega, J. M. Millam, M. Klene, J. E. Knox, J. B. Cross, V. Bakken, C. Adamo, J. Jaramillo, R. Gomperts, R. E. Stratmann, O. Yazyev, A. J. Austin, R. Cammi, C. Pomelli, J. W. Ochterski, R. L. Martin, K. Morokuma, V. G. Zakrzewski, G. A. Voth, P. Salvador, J. J. Dannenberg, S. Dapprich, A. D. Daniels, Farkas, J. B. Foresman, J. V. Ortiz, J. Cioslowski, D. J. Fox, Gaussian, Inc., Wallingford CT, 2009.

(158) Becke, A. D. Density-functional thermochemistry. III. The role of exact exchange. J. Chem. Phys. 1993, 98, 5648-52.

(159) Lee, C.; Yang, W.; Parr, R. G. Development of the Colle-Salvetti correlationenergy formula into a functional of the electron density. Phys. Rev. B: Condens. Matter 1988, 37, 785-9. 
(160) Vosko, S. H.; Wilk, L.; Nusair, M. Accurate spin-dependent electron liquid correlation energies for local spin density calculations: a critical analysis. Can. J. Phys. 1980, 58, 1200-11.

(161) Stephens, P. J.; Devlin, F. J.; Chabalowski, C. F.; Frisch, M. J. Ab Initio Calculation of Vibrational Absorption and Circular Dichroism Spectra Using Density Functional Force Fields. J. Phys. Chem. 1994, 98, 11623-7.

(162) Zhao, Y.; Truhlar, D. G. The M06 suite of density functionals for main group thermochemistry, thermochemical kinetics, noncovalent interactions, excited states, and transition elements: two new functionals and systematic testing of four M06-class functionals and 12 other functionals. Theor. Chem. Acc. 2008, 120, 215-241.

(163) Perez-Galan, P.; Herrero-Gomez, E.; Hog, D. T.; Martin, N. J. A.; Maseras, F.; Echavarren, A. M. Mechanism of the gold-catalyzed cyclopropanation of alkenes with 1,6-enynes. Chem. Sci. 2011, 2, 141-149.

(164) Gourlaouen, C.; Marion, N.; Nolan, S. P.; Maseras, F. Mechanism of the [(NHC)AuI]-Catalyzed Rearrangement of Allylic Acetates. A DFT Study. Org. Lett. 2009, 11, 81-84.

(165) Liu, Y.; Zhang, D.; Zhou, J.; Liu, C. Theoretical Elucidation of Au(I)-Catalyzed Cycloisomerizations of Cycloalkyl-substituted 1,5-Enynes: 1,2-alkyl Shift versus C-H Bond Insertion Products. J. Phys. Chem. A 2010, 114, 6164-6170.

(166) Paton, R. S.; Maseras, F. Gold(I)-Catalyzed Intermolecular Hydroalkoxylation of Allenes: A DFT study. Org. Lett. 2009, 11, 2237-2240. 
(167) La Londe, R. L.; Brenzovich, W. E., Jr.; Benitez, D.; Tkatchouk, E.; Kelley, K.; Goddard, W. A., III; Toste, F. D. Alkylgold complexes by the intramolecular aminoauration of unactivated alkenes. Chem. Sci. 2010, 1, 226-233.

(168) Krauter, C. M.; Hashmi, A. S. K.; Pernpointner, M. A new insight into gold(I)catalyzed hydration of alkynes: proton transfer. ChemCatChem 2010, 2, 1226-1230.

(169) Lein, M.; Rudolph, M.; Hashmi, A. S. K.; Schwerdtfeger, P. Homogeneous gold catalysis: mechanism and relativistic effects of the addition of water to propyne. Organometallics 2010, 29, 2206-2210.

(170) Hay, P. J.; Wadt, W. R. Ab initio effective core potentials for molecular calculations. Potentials for potassium to gold including the outermost core orbitals. J. Chem. Phys. 1985, 82, 299-310.

(171) Grimme, S. J. Chem. Phys. 2006, 124, 034108.

(172) Grimme, S. Improved second-order Moller-Plesset perturbation theory by separate scaling of parallel- and antiparallel-spin pair correlation energies. J. Chem. Phys. 2003, 118, 9095-9102.

(173) NBO Version 3.1, E. D. Glendening, A. E. Reed, J. E. Carpenter, F. Weinhold.

(174) Nevado, C.; Ferrer, C.; Echavarren, A. M. New Annulations via PlatinumCatalyzed Enyne Cyclization and Cyclopropane Cleavage. Org. Lett. 2004, 6, 3191-3194.

(175) Nieto-Oberhuber, C. M. B., E.; Nevado, C.; Curdenas, D. J.; Echavarren, A. M. Angew. Chem. Int. Ed. 2004, 43, 2311.

(176) Nieto-Oberhuber, C.; Perez-Galan, P.; Herrero-Gomez, E.; Lauterbach, T.; Rodriguez, C.; Lopez, S.; Bour, C.; Rosellon, A.; Cardenas, D. J.; Echavarren, A. M. 
Gold(I)-Catalyzed Intramolecular [4+2] Cycloadditions of Arylalkynes or 1,3-Enynes with Alkenes: Scope and Mechanism. J. Am. Chem. Soc. 2008, 130, 269-279.

(177) Brodmann, T. L., M.; Schaeckel, R.; Simsek, S.; Kalesse, M. Synlett 2009, 174.

(178) Schetter, B.; Mahrwald, R. Modern aldol methods for the total synthesis of polyketides. Angew. Chem., Int. Ed. 2006, 45, 7506-7525.

(179) Kimball, D. B.; Silks, L. A. III Current progress in the acetate/methyl ketone aldol reaction. Curr. Org. Chem. 2006, 10, 1975-1992.

(180) Feringa, B. L. Phosphoramidites: marvellous ligands in catalytic asymmetric conjugate addition. Acc. Chem. Res. 2000, 33, 346-53.

(181) Cowen, B. J.; Miller, S. J. Enantioselective catalysis and complexity generation from allenoates. Chem. Soc. Rev. 2009, 38, 3102-3116.

(182) Ma, S. Electrophilic Addition and Cyclization Reactions of Allenes. Acc. Chem. Res. 2009, 42, 1679-1688.

(183) Yang, Y.-Q.; Chai, Z.; Wang, H.-F.; Chen, X.-K.; Cui, H.-F.; Zheng, C.-W.; Xiao, H.; Li, P.; Zhao, G. Chiral Primary-Secondary Diamines Catalyzed Michael-AldolDehydration Reaction between Benzoylacetates and $\alpha, \beta$-Unsaturated Ketones: Highly Enantioselective Synthesis of Functionalized Chiral Cyclohexenones. Chem. - Eur. J. 2009, 15, 13295-13298.

(184) Carlone, A.; Marigo, M.; North, C.; Landa, A.; Jorgensen, K. A. A simple asymmetric organocatalytic approach to optically active cyclohexenones. Chem. Commun. (Cambridge, U. K.) 2006, 4928-4930. 
(185) Bolze, P.; Dickmeiss, G.; Joergensen, K. A. Organocatalytic Asymmetric Synthesis of 5-(Trialkylsilyl)cyclohex-2-enones and the Transformation into Useful Building Blocks. Org. Lett. 2008, 10, 3753-3756.

(186) Albrecht, L.; Richter, B.; Villa, C.; Krawczyk, H.; Joergensen, K. A. Organocatalytic domino Michael-Knoevenagel condensation reaction for the synthesis of optically active 3-diethoxyphosphoryl-2-oxocyclohex-3-enecarboxylates. Chem. - Eur. J. 2009, 15, 3093-3102.

(187) Halland, N.; Aburel, P. S.; Jorgensen, K. A. Highly enantio- and diastereoselective organocatalytic asymmetric domino Michael-Aldol reaction of $\beta$-keto esters and $\alpha, \beta$-unsaturated ketones. Angew. Chem., Int. Ed. 2004, 43, 1272-1277.

(188) Hayashi, Y.; Toyoshima, M.; Gotoh, H.; Ishikawa, H. Diphenylprolinol Silyl Ether Catalysis in an Asymmetric Formal Carbo [3 + 3] Cycloaddition Reaction via a Domino Michael/Knoevenagel Condensation. Org. Lett. 2009, 11, 45-48.

(189) Xie, J.-W.; Chen, W.; Li, R.; Zeng, M.; Du, W.; Yue, L.; Chen, Y.-C.; Wu, Y.; Zhu, J.; Deng, J.-G. Highly asymmetric Michael addition to $\alpha, \beta$-unsaturated ketones catalyzed by 9-amino-9-deoxyepiquinine. Angew. Chem., Int. Ed. 2007, 46, 389-392.

(190) Klunder, A. J. H.; Zhu, J.; Zwanenburg, B. The Concept of Transient Chirality in the Stereoselective Synthesis of Functionalized Cycloalkenes Applying the Retro-DielsAlder Methodology. Chem. Rev. (Washington, D. C.) 1999, 99, 1163-1190.

(191) Mohr, P. J.; Halcomb, R. L. Total Synthesis of (+)-Phomactin A Using a B-Alkyl Suzuki Macrocyclization. J. Am. Chem. Soc. 2003, 125, 1712-1713. 
(192) Miyashita, M.; Sasaki, M.; Hattori, I.; Sakai, M.; Tanino, K. Total Synthesis of Norzoanthamine. Science (Washington, DC, U. S.) 2004, 305, 495-499.

(193) Baran, P. S.; Richter, J. M.; Lin, D. W. Direct coupling of pyrroles with carbonyl compounds: Short enantioselective synthesis of (S)-ketorolac. Angew. Chem., Int. Ed. 2005, 44, 609-612.

(194) Goeke, A.; Mertl, D.; Brunner, G. Alkyl aluminum halide promoted intramolecular cyclization of $\omega$-allyl cycloalk-2-enones: Access to bridged bi- and tricyclic compounds. Angew. Chem., Int. Ed. 2005, 44, 99-101.

(195) Lakshmi, R.; Bateman, T. D.; McIntosh, M. C. A Convenient 3-Step Synthesis of (R)-7-Hydroxycarvone from (S)- $\alpha$-Pinene. J. Org. Chem. 2005, 70, 5313-5315.

(196) Zhou, J.; Wakchaure, V.; Kraft, P.; List, B. Primary-amine-catalyzed enantioselective intramolecular aldolizations. Angew. Chem., Int. Ed. 2008, 47, 76567658.

(197) Swiss, K. A.; Liotta, D. C.; Maryanoff, C. A. Mechanistic aspects of the ligandassisted nucleophilic addition reaction. J. Am. Chem. Soc. 1990, 112, 9393-4.

(198) Swiss, K. A. H., W.; Maryanoff, C. A.; Liotta, D. C. Synthesis 1992, 127.

(199) Semmelhack, M. F.; Gallagher, J. J.; Minami, T.; Date, T. The enol-keto trigger in initiating arene diradical formation in calicheamicin/esperamicin analogs. J. Am. Chem. Soc. 1993, 115, 11618-19.

(200) Kitagaki, S.; Teramoto, S.; Ohta, Y.; Kobayashi, H.; Takabe, M.; Mukai, C. Chemistry of allenic/propargylic anions generated by base treatment of sulfonylallenes: 
synthesis of 1-alkynyl-1-sulfonylcycloalkanes and cycloalkanols. Tetrahedron 2010, 66, 3687-3694.

(201) Geoffroy, P.; Ballet, M. P.; Finck, S.; Marchioni, E.; Miesch, M. Domino reactions starting from alkynyl esters tethered to 2-methyl-1,3-cycloalkanediones. Efficient access to polyfunctionalized diquinanes, allenoates, and oxetanes. Tetrahedron 2010, 66, 7012-7016.

(202) Paike, V. V.; Balakumar, R.; Chen, H.-Y.; Shih, H.-P.; Han, C.-C. A Serendipitous C-C Bond Formation Reaction between Michael Donors and Diiminoquinoid Ring Assisted by Quaternary Ammonium Fluoride. Org. Lett. 2009, 11, $5586-5589$.

(203) Srihari, P.; Dutta, P.; Rao, R. S.; Yadav, J. S.; Chandrasekhar, S.; Thombare, P.; Mohapatra, J.; Chatterjee, A.; Jain, M. R. Solvent free synthesis of 1,5-disubstituted tetrazoles derived from Baylis Hillman acetates as potential TNF- $\alpha$ inhibitors. Bioorg. Med. Chem. Lett. 2009, 19, 5569-5572.

(204) Aponte, J. C.; Hammond, G. B.; Xu, B. TBAF-Mediated Aldol Reaction of $\beta$ Allenoates: Regio- and Stereoselective Synthesis of (2E,4E)-4-Carbinol Alkadienoates. $J$. Org. Chem. 2009, 74, 4623-4625.

(205) Miesch, L.; Welsch, T.; Rietsch, V.; Miesch, M. Intramolecular Alkynylogous Mukaiyama Aldol Reaction Starting from Bicyclic Alkanones Tethered to Alkynyl Esters: Formal Total Synthesis of ( \pm )-Hamigeran B. Chem. - Eur. J. 2009, 15, 4394-4401. 
(206) Kitagaki, S.; Teramoto, S.; Mukai, C. Ring-Closing Reaction of Allenic/Propargylic Anions Generated by Base Treatment of Sulfonylallenes. Org. Lett. 2007, 9, 2549-2552.

(207) Mota, A. J.; Klein, A.; Wendling, F.; Dedieu, A.; Miesch, M. Controlled Synthesis of $\alpha$-Allenic Ester and Spiro Ketone Derivatives from Tailored $\alpha$-Substituted Cycloalkanones Through Cascade Reactions: Exploring the Possible Reaction Pathways by Means of Semiempirical MO Calculations. Eur. J. Org. Chem. 2005, 4346-4358.

(208) Wendling, F.; Miesch, M. Unexpected Reactivity of Acetylenic $\omega$-Ketoesters toward TBAF and t-BuOK; New Cascade Reactions Affording Allene and Oxetane Derivatives. Org. Lett. 2001, 3, 2689-2691.

(209) Hashimoto, T.; Maruoka, K. Recent Development and Application of Chiral Phase-Transfer Catalysts. Chem. Rev. (Washington, DC, U. S.) 2007, 107, 5656-5682.

(210) Jew, S.-s.; Park, H.-g. Cinchona-based phase-transfer catalysts for asymmetric synthesis. Chem. Commun. (Cambridge, U. K.) 2009, 7090-7103.

(211) Hashimoto, T.; Sakata, K.; Maruoka, K. $\alpha$-Chiral Acetylenes Having an AllCarbon Quaternary Center: Phase Transfer Catalyzed Enantioselective $\alpha$ Alkylation of $\alpha$ Alkyl- $\alpha$-alkynyl Esters. Angew. Chem., Int. Ed. 2009, 48, 5014-5017.

(212) The ${ }^{1} \mathrm{H}$ NMR spectra of compounds 3-3j, 3-3k, and 3-31 contain traces of olefinic impurity which might derive from ethyl vinyl ketone.

(213) Yeom, H.-S.; Koo, J.; Park, H.-S.; Wang, Y.; Liang, Y.; Yu, Z.-X.; Shin, S. GoldCatalyzed Intermolecular Reactions of Propiolic Acids with Alkenes: [4 + 2] Annulation and Enyne Cross Metathesis. J. Am. Chem. Soc. 2012, 134, 208-211. 
(214) Brown, T. J.; Weber, D.; Gagne, M. R.; Widenhoefer, R. A. Mechanistic Analysis of Gold(I)-Catalyzed Intramolecular Allene Hydroalkoxylation Reveals an Off-Cycle Bis(gold) Vinyl Species and Reversible C-O Bond Formation. J. Am. Chem. Soc. 2012, $134,9134-9137$.

(215) Lopez-Carrillo, V.; Echavarren, A. M. Gold(I)-Catalyzed Intermolecular [2+2] Cycloaddition of Alkynes with Alkenes. J. Am. Chem. Soc. 2010, 132, 9292-9294.

(216) Li, H.; Widenhoefer, R. A. Gold(I)-Catalyzed Intramolecular Dihydroamination of Allenes with N,N'-Disubstituted Ureas To Form Bicyclic Imidazolidin-2-ones. Org. Lett. 2009, 11, 2671-2674.

(217) Zhang, Z.; Bender, C. F.; Widenhoefer, R. A. Gold(I)-Catalyzed Dynamic Kinetic Enantioselective Intramolecular Hydroamination of Allenes. J. Am. Chem. Soc. 2007, 129, 14148-14149.

(218) Ito, Y.; Sawamura, M.; Hayashi, T. Catalytic asymmetric aldol reaction: reaction of aldehydes with isocyanoacetate catalyzed by a chiral ferrocenylphosphine-gold(I) complex. J. Am. Chem. Soc. 1986, 108, 6405-6.

(219) He, W.; Li, C.; Zhang, L. An Efficient [2+2+1] Synthesis of 2,5-Disubstituted Oxazoles via Gold-Catalyzed Intermolecular Alkyne Oxidation. J. Am. Chem. Soc. 2011, 133, 8482-8485.

(220) Winter, C.; Krause, N. Structural Diversity through Gold Catalysis: Stereoselective Synthesis of N-Hydroxypyrrolines, Dihydroisoxazoles, and Dihydro-1,2oxazines. Angew. Chem., Int. Ed. 2009, 48, 6339-6342. 
(221) Corma, A.; Leyva-Perez, A.; Sabater, M. J. Gold-catalyzed carbon-heteroatom bond-forming reactions. Chem. Rev. 2011, 111, 1657-712.

(222) Saito, A.; Konishi, T.; Hanzawa, Y. Synthesis of pyrroles by gold(I)-catalyzed amino-Claisen rearrangement of N-propargyl enaminone derivatives. Org. Lett. 2010, 12, $372-374$.

(223) Wang, S.; Zhang, L. A Gold-Catalyzed Unique Cycloisomerization of 1,5-Enynes: Efficient Formation of 1-Carboxycyclohexa-1,4-dienes and Carboxyarenes. J. Am. Chem. Soc. 2006, 128, 14274-14275.

(224) Wang, S.; Zhang, L. A Highly Efficient Preparative Method of $\alpha$-Ylidene- $\beta$ Diketones via AuIII-Catalyzed Acyl Migration of Propargylic Esters. J. Am. Chem. Soc. 2006, 128, 8414-8415.

(225) Dar, A.; Moss, K.; Cottrill, S. M.; Parish, R. V.; McAuliffe, C. A.; Pritchard, R. G.; Beagley, B.; Sandbank, J. Complexes of gold(III) and mononegative bidentate N,Oligands. J. Chem. Soc., Dalton Trans. 1992, 1907-13.

(226) Clavier, H.; Nolan, S. P. Percent buried volume for phosphine and N-heterocyclic carbene ligands: steric properties in organometallic chemistry. Chem. Commun. (Cambridge, U. K.) 2010, 46, 841-861.

(227) Hashmi, A. S. K.; Lothschuetz, C.; Boehling, C.; Hengst, T.; Hubbert, C.; Rominger, F. Carbenes Made Easy: formation of Unsymmetrically Substituted NHeterocyclic Carbene Complexes of Palladium(II), Platinum(II) and Gold(I) from Coordinated Isonitriles and their Catalytic Activity. Adv. Synth. Catal. 2010, 352, 30013012. 
(228) Pazicky, M.; Loos, A.; Ferreira, M. J.; Serra, D.; Vinokurov, N.; Rominger, F.; Jakel, C.; Hashmi, A. S. K.; Limbach, M. Synthesis, Reactivity, and Electrochemical Studies of Gold(I) and Gold(III) Complexes Supported by N-Heterocyclic Carbenes and Their Application in Catalysis. Organometallics 2010, 29, 4448-4458.

(229) Hashmi, A. S. K.; Hengst, T.; Lothschuetz, C.; Rominger, F. New and Easily Accessible Nitrogen Acyclic Gold(I) Carbenes: Structure and Application in the GoldCatalyzed Phenol Synthesis as well as the Hydration of Alkynes. Adv. Synth. Catal. 2010, 352, $1315-1337$.

(230) Bartolome, C.; Ramiro, Z.; Garcia-Cuadrado, D.; Perez-Galan, P.; Raducan, M.; Bour, C.; Echavarren, A. M.; Espinet, P. Nitrogen Acyclic Gold(I) Carbenes: Excellent and Easily Accessible Catalysts in Reactions of 1,6-Enynes. Organometallics 2010, 29, 951-956.

(231) Bartolome, C.; Garcia-Cuadrado, D.; Ramiro, Z.; Espinet, P. Exploring the Scope of Nitrogen Acyclic Carbenes (NACs) in Gold-Catalyzed Reactions. Organometallics 2010, 29, 3589-3592.

(232) Alcarazo, M.; Stork, T.; Anoop, A.; Thiel, W.; Fuerstner, A. Steering the Surprisingly Modular $\pi$-Acceptor Properties of N-Heterocyclic Carbenes: Implications for Gold Catalysis. Angew. Chem., Int. Ed. 2010, 49, 2542-2546.

(233) Bantreil, X.; Broggi, J.; Nolan, S. P. N-Heterocyclic carbene containing complexes in catalysis. Annu. Rep. Prog. Chem., Sect. B: Org. Chem. 2009, 105, 232263. 
(234) Diez-Gonzalez, S.; Marion, N.; Nolan, S. P. N-Heterocyclic Carbenes in Late Transition Metal Catalysis. Chem. Rev. (Washington, DC, U. S.) 2009, 109, 3612-3676.

(235) Aldeco-Perez, E.; Rosenthal, A. J.; Donnadieu, B.; Parameswaran, P.; Frenking, G.; Bertrand, G. Isolation of a C5-Deprotonated Imidazolium, a Crystalline "Abnormal" N-Heterocyclic Carbene. Science (Washington, DC, U. S.) 2009, 326, 556-559.

(236) Bartolome, C.; Ramiro, Z.; Perez-Galan, P.; Bour, C.; Raducan, M.; Echavarren, A. M.; Espinet, P. Gold(I) Complexes with Hydrogen-Bond Supported Heterocyclic Carbenes as Active Catalysts in Reactions of 1,6-Enynes. Inorg. Chem. 2008, 47, 1139111397.

(237) Kinder, R. E.; Zhang, Z.; Widenhoefer, R. A. Intermolecular Hydroamination of Allenes with N-Unsubstituted Carbamates Catalyzed by a Gold(I) N-Heterocyclic Carbene Complex. Org. Lett. 2008, 10, 3157-3159.

(238) Marion, N.; Carlqvist, P.; Gealageas, R.; de Fremont, P.; Maseras, F.; Nolan, S. P. [(NHC)AuI]-catalyzed formation of conjugated enones and enals: An experimental and computational study. Chem. - Eur. J. 2007, 13, 6437-6451.

(239) Marion, N.; Diez-Gonzalez, S.; de Fremont, P.; Noble, A. R.; Nolan, S. P. Au1catalyzed tandem [3,3] rearrangement-intramolecular hydroarylation: mild and efficient formation of substituted indenes. Angew. Chem., Int. Ed. 2006, 45, 3647-3650.

(240) Bourissou, D.; Guerret, O.; Gabbaie, F. P.; Bertrand, G. Stable Carbenes. Chemical Reviews (Washington, D. C.) 2000, 100, 39-91.

(241) Lee, A.-L. Enantioselective catalysis. Annu. Rep. Prog. Chem., Sect. B: Org. Chem. 2011, 107, 369-389. 
(242) Ferrer, C.; Amijs, C. H. M.; Echavarren, A. M. Intra- and intermolecular reactions of indoles with alkynes catalyzed by gold. Chem. - Eur. J. 2007, 13, 1358-1373.

(243) Herrero-Gomez, E.; Nieto-Oberhuber, C.; Lopez, S.; Benet-Buchholz, J.; Echavarren, A. M. Cationic $\eta^{1} / \eta^{2}$-gold(I) complexes of simple arenes. Angew. Chem., Int. Ed. 2006, 45, 5455-5459.

(244) Ferrer, C.; Echavarren, A. M. Gold-catalyzed intramolecular reaction of indoles with alkynes: facile formation of eight-membered rings and an unexpected allenylation. Angew. Chem., Int. Ed. 2006, 45, 1105-1109.

(245) Istrate, F. M.; Gagosz, F. Synthesis of functionalized pyrroles via gold(I)catalyzed aza-Claisen-type rearrangement. Org. Lett. 2007, 9, 3181-3184.

(246) Nieto-Oberhuber, C.; Munoz, M. P.; Lopez, S.; Jimenez-Nunez, E.; Nevado, C.; Herrero-Gomez, E.; Raducan, M.; Echavarren, A. M. Gold(I)-catalyzed cyclizations of 1,6-enynes: alkoxycyclizations and exo/endo skeletal rearrangements. Chem. - Eur. J. 2006, 12, 1677-1693.

(247) Nieto-Oberhuber, C.; Lopez, S.; Echavarren, A. M. Intramolecular [4+2] cycloadditions of 1,3-enynes or arylalkynes with alkenes with highly reactive cationic phosphine Au(I) complexes. J. Am. Chem. Soc. 2005, 127, 6178-6179.

(248) Walker, S. D.; Barder, T. E.; Martinelli, J. R.; Buchwald, S. L. A rationally designed universal catalyst for Suzuki-Miyaura coupling processes. Angew. Chem., Int. Ed. 2004, 43, 1871-1876.

(249) Strieter, E. R.; Blackmond, D. G.; Buchwald, S. L. Insights into the Origin of High Activity and Stability of Catalysts Derived from Bulky, Electron-Rich 
Monophosphinobiaryl Ligands in the Pd-Catalyzed C-N Bond Formation. J. Am. Chem. Soc. 2003, 125, 13978-13980.

(250) Kaye, S.; Fox, J. M.; Hicks, F. A.; Buchwald, S. L. The use of catalytic amounts of $\mathrm{CuCl}$ and other improvements in the benzyne route to biphenyl-based phosphine ligands. Adv. Synth. Catal. 2001, 343, 789-794.

(251) Wang, Q.; Aparaj, S.; Akhmedov, N. G.; Petersen, J. L.; Shi, X. Ambient Schmittel cyclization promoted by chemoselective triazole-gold catalyst. Org. Lett. 2012, 14, 1334-1337.

(252) Wang, D.; Gautam, L. N. S.; Bollinger, C.; Harris, A.; Li, M.; Shi, X. 1,2,3Triazole Bound $\mathrm{Au}(\mathrm{I})(\mathrm{TA}-\mathrm{Au})$ as Chemoselective Catalysts in Promoting Asymmetric Synthesis of Substituted Allenes. Org. Lett. 2011, 13, 2618-2621.

(253) Wang, D.; Zhang, Y.; Cai, R.; Shi, X. Triazole-Au(I) complex as chemoselective catalyst in promoting propargyl ester rearrangements. Beilstein J. Org. Chem. 2011, 7, 1014-1020, No. 115.

(254) Wang, D.; Zhang, Y.; Harris, A.; Gautam, L. N. S.; Chen, Y.; Shi, X. Triazolegold-promoted, effective synthesis of enones from propargylic esters and alcohols: A catalyst offering chemoselectivity, acidity and ligand economy. Adv. Synth. Catal. 2011, $353,2584-2588$.

(255) Wang, D.; Ye, X.; Shi, X. Efficient synthesis of E- $\alpha$-haloenones through chemoselective alkyne activation over allene with triazole-Au catalysts. Org. Lett. 2010, 12, 2088-2091. 
(256) Chen, Y.; Wang, D.; Petersen, J. L.; Akhmedov, N. G.; Shi, X. Synthesis and characterization of organogold complexes containing an acid stable Au-C bond through triazole-yne 5-endo-dig cyclization. Chem. Commun. 2010, 46, 6147-6149.

(257) Yan, W.; Wang, Q.; Chen, Y.; Petersen, J. L.; Shi, X. Iron-Catalyzed C-O Bond Activation for the Synthesis of Propargyl-1,2,3-triazoles and 1,1-Bis-triazoles. Org. Lett. 2010, 12, 3308-3311.

(258) Ricard, L.; Gagosz, F. Synthesis and Reactivity of Air-Stable N-Heterocyclic Carbene Gold(I) Bis(trifluoromethanesulfonyl)imidate Complexes. Organometallics 2007, 26, 4704-4707.

(259) Buzas, A. K.; Istrate, F. M.; Gagosz, F. Gold(I)-catalyzed 5-endo hydroxy- and alkoxycyclization of 1,5-enynes: efficient access to functionalized cyclopentenes. Angew. Chem., Int. Ed. 2007, 46, 1141-1144.

(260) Buzas, A.; Gagosz, F. Gold(I)-Catalyzed Formation of 4-Alkylidene-1,3dioxolan-2-ones from Propargylic tert-Butyl Carbonates. Org. Lett. 2006, 8, 515-518.

(261) Buzas, A.; Gagosz, F. Gold(I) Catalyzed Isomerization of 5-en-2-yn-1-yl Acetates: An Efficient Access to Acetoxy Bicyclo[3.1.0]hexenes and 2-Cycloalken-1-ones. J. Am. Chem. Soc. 2006, 128, 12614-12615.

(262) Mezailles, N.; Ricard, L.; Gagosz, F. Phosphine Gold(I) Bis(trifluoromethanesulfonyl)imidate Complexes as New Highly Efficient and Air-Stable Catalysts for the Cycloisomerization of Enynes. Org. Lett. 2005, 7, 4133-4136. 
(263) Gonzalez, A. Z.; Benitez, D.; Tkatchouk, E.; Goddard, W. A., III; Toste, F. D. Phosphoramidite Gold(I)-Catalyzed Diastereo- and Enantioselective Synthesis of 3,4Substituted Pyrrolidines. J. Am. Chem. Soc. 2011, 133, 5500-5507.

(264) Gonzalez, A. Z.; Toste, F. D. Gold(I)-Catalyzed Enantioselective [4+2]Cycloaddition of Allene-dienes. Org. Lett. 2010, 12, 200-203.

(265) Teller, H.; Fluegge, S.; Goddard, R.; Fuerstner, A. Enantioselective Gold Catalysis: Opportunities Provided by Monodentate Phosphoramidite Ligands with an Acyclic TADDOL Backbone. Angew. Chem., Int. Ed. 2010, 49, 1949-1953.

(266) Alonso, I.; Trillo, B.; Lopez, F.; Montserrat, S.; Ujaque, G.; Castedo, L.; Lledos, A.; Mascarenas, J. L. Gold-catalyzed [4C $+2 \mathrm{C}]$ cycloadditions of allenedienes, including an enantioselective version with new phosphoramidite-based catalysts: mechanistic aspects of the divergence between $[4 \mathrm{C}+3 \mathrm{C}]$ and $[4 \mathrm{C}+2 \mathrm{C}]$ pathways. J. Am. Chem. Soc. 2009, 131, 13020-13030.

(267) Hamilton, G. L.; Kang, E. J.; Mba, M.; Toste, F. D. A powerful chiral counterion strategy for asymmetric transition metal catalysis. Science 2007, 317, 496-499.

(268) Melhado, A. D.; Luparia, M.; Toste, F. D. Au(I)-catalyzed enantioselective 1,3dipolar cycloadditions of munchnones with electron-deficient alkenes. J. Am. Chem. Soc. 2007, 129, 12638-12639.

(269) LaLonde, R. L.; Sherry, B. D.; Kang, E. J.; Toste, F. D. Gold(I)-Catalyzed Enantioselective Intramolecular Hydroamination of Allenes. J. Am. Chem. Soc. 2007, 129, 2452-2453. 
(270) Gaillard, S.; Cazin, C. S. J.; Nolan, S. P. N-Heterocyclic Carbene Gold(I) and Copper(I) Complexes in C-H Bond Activation. Acc. Chem. Res. 2012, 45, 778-787.

(271) Boogaerts, I. I. F.; Nolan, S. P. Carboxylation of C-H Bonds Using NHeterocyclic Carbene Gold(I) Complexes. J. Am. Chem. Soc. 2010, 132, 8858-8859.

(272) Gao, K.; Wu, J. Synthesis of Functionalized 1,2-Dihydroisoquinolines via Multicomponent One-Pot Reaction of 2-Alkynylbenzaldehyde, Amine, Zinc, and Allylic Bromide or Benzyl Bromide. J. Org. Chem. 2007, 72, 8611-8613.

(273) Zheng, D.; Li, S.; Wu, J. An Unexpected Silver Triflate Catalyzed Reaction of 2Alkynylbenzaldehyde with 2-Isocyanoacetate. Org. Lett. 2012, 14, 2655-2657.

(274) Li, S.; Wu, J. Synthesis of H-Pyrazolo[5,1-a]isoquinolines via Copper(II)Catalyzed Oxidation of an Aliphatic C-H Bond of Tertiary Amine in Air. Org. Lett. 2011, $13,712-715$.

(275) Markina, N. A.; Mancuso, R.; Neuenswander, B.; Lushington, G. H.; Larock, R. C. Solution-Phase Parallel Synthesis of a Diverse Library of 1,2-Dihydroisoquinolines. ACS Comb. Sci. 2011, 13, 265-271.

(276) Chen, Z.; Wu, J. Efficient generation of biologically active H-pyrazolo[5,1a]isoquinolines via multicomponent reaction. Org. Lett. 2010, 12, 4856-4859.

(277) Ding, Q.; Wu, J. Lewis Acid- and Organocatalyst-Cocatalyzed Multicomponent Reactions of 2-Alkynylbenzaldehydes, Amines, and Ketones. Org. Lett. 2007, 9, 49594962.

(278) Sun, W.; Ding, Q.; Sun, X.; Fan, R.; Wu, J. AgOTf-catalyzed three-component reactions of 2-alkynylbenzaldehydes, amines, and diethylphosphite. an efficient route to 
2,3-disubstituted-1,2-dihydroisoquinolin-1-ylphosphonates. J. Comb. Chem. 2007, 9, 690-694.

(279) Yao, X.; Li, C.-J. Water-Triggered and Gold(I)-Catalyzed Cascade Addition/Cyclization of Terminal Alkynes with ortho-Alkynylaryl Aldehyde. Org. Lett. 2006, 8, 1953-1955.

(280) Asao, N.; Sato, K.; Menggenbateer; Yamamoto, Y. AuBr3- and $\mathrm{Cu}(\mathrm{OTf})_{2-}$ Catalyzed Intramolecular [4 +2$]$ Cycloaddition of Tethered Alkynyl and Alkenyl Enynones and Enynals: A New Synthetic Method for Functionalized Polycyclic Hydrocarbons. J. Org. Chem. 2005, 70, 3682-3685.

(281) Asao, N.; Chan, C. S.; Takahashi, K.; Yamamoto, Y. Domino allylation and cyclization of ortho-alkynylbenzaldehydes with allyltrimethylsilane catalyzed by $\mathrm{Pd}(\mathrm{II})$ $\mathrm{Cu}(\mathrm{II})$ bimetallic systems. Tetrahedron 2005, 61, 11322-11326.

(282) Patil, N. T.; Pahadi, N. K.; Yamamoto, Y. Silver(I)-catalyzed novel cascade cyclization reactions: Incorporation of allenes into the isochromenes. J. Org. Chem. 2005, 70, 10096-10098.

(283) Sato, K.; Asao, N.; Yamamoto, Y. Efficient Method for Synthesis of Angucyclinone Antibiotics via Gold-Catalyzed Intramolecular [4 +2] Benzannulation: Enantioselective Total Synthesis of (+)-Ochromycinone and (+)-Rubiginone B2. J. Org. Chem. 2005, 70, 8977-8981.

(284) Döpp, R.; Lothschütz, C.; Wurm, T.; Pernpointner, M.; Keller, S.; Rominger, F.; Hashmi, A. S. K. Gold Catalysis: Hydrolysis of Di(alkoxy)carbenium Ion Intermediates 
as a Sensor for the Electronic Properties of Gold(I) Complexes. Organometallics 2011, 30, 5894-5903.

(285) Benitez, D.; Shapiro, N. D.; Tkatchouk, E.; Wang, Y.; Goddard, W. A., III; Toste, F. D. A bonding model for gold(I) carbene complexes. Nature Chemistry 2009, 1, 482486.

(286) Liu, L.-P.; Malhotra, D.; Jin, Z.; Paton, R. S.; Houk, K. N.; Hammond, G. B. Gold-Catalyzed, Intramolecular, Oxygen-Transfer Reactions of 2-Alkynyl-1,5-diketones or 2-Alkynyl-5-ketoesters: Scope, Expansion, and Mechanistic Investigations on a New [4+2] Cycloaddition. Chem. - Eur. J. 2011, 17, 10690-10699.

(287) Liu, L.-P.; Malhotra, D.; Paton, R. S.; Houk, K. N.; Hammond, G. B. The [4+2], not [2+2], Mechanism Occurs in the Gold-Catalyzed Intramolecular Oxygen Transfer Reaction of 2-Alkynyl-1,5-Diketones. Angew. Chem., Int. Ed. 2010, 49, 9132-9135.

(288) Magnus, P.; Sane, N.; Fauber, B. P.; Lynch, V. Concise Syntheses of (-)Galanthamine and $( \pm)$-Codeine via Intramolecular Alkylation of a Phenol Derivative. $J$. Am. Chem. Soc. 2009, 131, 16045-16047.

(289) Shishido, Y.; Wakabayashi, H.; Koike, H.; Ueno, N.; Nukui, S.; Yamagishi, T.; Murata, Y.; Naganeo, F.; Mizutani, M.; Shimada, K.; Fujiwara, Y.; Sakakibara, A.; Suga, O.; Kusano, R.; Ueda, S.; Kanai, Y.; Tsuchiya, M.; Satake, K. Discovery and stereoselective synthesis of the novel isochroman neurokinin-1 receptor antagonist CJ17,493'. Bioorg. Med. Chem. 2008, 16, 7193-7205. 
(290) Hayashi, T.; Smith, F. T.; Lee, K. H. Antitumor agents. 89. Psychorubrin, a new cytotoxic naphthoquinone from Psychotria rubra and its structure-activity relationships. $J$. Med. Chem. 1987, 30, 2005-8.

(291) Lieberman, M. E.; Gorski, J.; Jordan, V. C. An estrogen receptor model to describe the regulation of prolactin synthesis by antiestrogens in vitro. J. Biol. Chem. 1983, 258, 4741-5.

(292) Yusupov, M. K.; Abdullaeva, D. A.; Aslanov, K. A.; Sadykov, A. S. The structure of regalamine. Chem. Nat. Compd. 1975, 11, 395-397.

(293) Perchellet, E. M.; Wang, Y.; Weber, R. L.; Lou, K.; Hua, D. H.; Perchellet, J.-P. H. Antitumor triptycene bisquinones induce a caspase-independent release of mitochondrial cytochrome $\mathrm{c}$ and a caspase-2-mediated activation of initiator caspase-8 and -9 in HL-60 cells by a mechanism which does not involve Fas signaling. Anti-Cancer Drugs 2004, 15, 929-946.

(294) Wang, B.; Perchellet, E. M.; Wang, Y.; Tamura, M.; Hua, D. H.; Perchellet, J.-P. H. Antitumor triptycene bisquinones: a novel synthetic class of dual inhibitors of DNA topoisomerase I and II activities. Anti-Cancer Drugs 2003, 14, 503-514.

(295) Liu, Y.; Ma, S. Benzofurans or Isochromenes via the Ring-Opening Cyclization of Cyclopropene Derivatives with Organolithiums. Org. Lett. 2012, 14, 720-723.

(296) Morimoto, K.; Hirano, K.; Satoh, T.; Miura, M. Synthesis of isochromene and related derivatives by rhodium-catalyzed oxidative coupling of benzyl and allyl alcohols with alkynes. J. Org. Chem. 2011, 76, 9548-9551. 
(297) Lu, D.-F.; Zhou, Y.-R.; Li, Y.-J.; Yan, S.-B.; Gong, Y.-F. Copper(II)-Catalyzed Asymmetric Henry Reaction of o-Alkynylbenzaldehydes Followed by Gold(I)-Mediated Cycloisomerization: An Enantioselective Route to Chiral 1H-Isochromenes and 1,3Dihydroisobenzofurans. J. Org. Chem. 2011, 76, 8869-8878.

(298) Zhou, M.-B.; Wei, W.-T.; Xie, Y.-X.; Lei, Y.; Li, J.-H. Palladium-catalyzed cross-coupling of electron-poor terminal alkynes with arylboronic acids under ligand-free and aerobic conditions. J. Org. Chem. 2010, 75, 5635-5642.

(299) Mancuso, R.; Mehta, S.; Gabriele, B.; Salerno, G.; Jenks, W. S.; Larock, R. C. A simple and mild synthesis of 1H-isochromenes and (Z)-1-alkylidene-1,3dihydroisobenzofurans by the iodocyclization of 2-(1-alkynyl)benzylic alcohols. J. Org. Chem. 2010, 75, 897-901.

(300) Ley, S. V.; Mynett, D. M.; Koot, W.-J. Solid phase synthesis of bicyclo[2.2.2]octane derivatives via tandem Michael addition reactions and subsequent reductive amination. Synlett 1995, 1017-20.

(301) Yang, H.; Carter, R. G. Organocatalyzed, enantioselective synthesis of bicyclo[2.2.2]-octanes containing benzylic, all-carbon quaternary centers. Tetrahedron 2010, 66, 4854-4859.

(302) Foubelo, F.; Moreno, B.; Yus, M. Reductive opening of $1 \mathrm{H}, 3 \mathrm{H}-$ benzo[de]isochromene: synthesis of 1,8-difunctionalized naphthalenes. Tetrahedron 2004, $60,4655-4662$.

(303) Jiménez-Núñez, E.; Claverie, C. K.; Bour, C.; Cárdenas, D. J.; Echavarren, A. M. cis-Selective Single-Cleavage Skeletal Rearrangement of 1,6-Enynes Reveals the 
Multifaceted Character of the Intermediates in Metal-Catalyzed Cycloisomerizations. Angew. Chem. 2008, 120, 8010-8013.

(304) Munoz, M. P.; Adrio, J.; Carretero, J. C.; Echavarren, A. M. Ligand Effects in Gold- and Platinum-Catalyzed Cyclization of Enynes: Chiral Gold Complexes for Enantioselective Alkoxycyclization. Organometallics 2005, 24, 1293-1300.

(305) Eliason, R.; Kreevoy, M. M. Comparison of general-acid-catalyzed ethyl vinyl ether hydrolysis in $80 \%$ dimethyl sulfoxide with that in water. J. Phys. Chem. 1974, 78, 2658-9.

(306) Kreevoy, M. M.; Eliason, R. Broensted $\alpha$ and isotope effects for vinyl ether hydrolysis. J. Phys. Chem. 1968, 72, 1313-16.

(307) Fananas, F. J.; Mendoza, A.; Arto, T.; Temelli, B.; Rodriguez, F. Scalable Total Synthesis of (-)-Berkelic Acid Using a Protecting-Group-Free Strategy. Angew. Chem., Int. Ed. 2012, 51, 4930-4933.

(308) Asao, N.; Menggenbateer; Seya, Y.; Yamamoto, Y.; Chen, M.; Zhang, W.; Inoue, A. Nanoporous gold-catalyzed $\left[\begin{array}{lll}4 & + & 2\end{array}\right]$ benzannulation between orthoalkynylbenzaldehydes and alkynes. Synlett 2012, 23, 66-69.

(309) Aikawa, H.; Tago, S.; Umetsu, K.; Haginiwa, N.; Asao, N. Gold-catalyzed substitution reaction with ortho-alkynylbenzoic acid alkyl ester as an efficient alkylating agent. Tetrahedron 2009, 65, 1774-1784.

(310) Sato, K.; Menggenbateer; Kubota, T.; Asao, N. AuCl-catalyzed reaction of orthoalkynyl(oxo)benzene with benzenediazonium 2-carboxylate as a synthetic method 
towards anthracene, triptycene, and phthalazine derivatives. Tetrahedron 2007, 64, 787796.

(311) Asao, N.; Aikawa, H. Lewis acid-catalyzed [4 +2$]$ benzannulation between enynal units and enols or enol ethers: Novel synthetic tools for polysubstituted aromatic compounds including indole and benzofuran derivatives. J. Org. Chem. 2006, 71, 52495253.

(312) Asao, N.; Sato, K. AuCl-catalyzed [4 +2$]$ benzannulation between oalkynyl(oxo)benzene and benzyne. Org. Lett. 2006, 8, 5361-5363.

(313) Under acidic conditions, ethyl vinyl ether is sensitive and could serve as source of ethanol along with intermediate E.

(314) Perez-Galan, P.; Delpont, N.; Herrero-Gomez, E.; Maseras, F.; Echavarren, A. M. Metal-arene interactions in dialkylbiarylphosphane complexes of copper, silver, and gold. Chem. - Eur. J. 2010, 16, 5324-5332.

(315) Hashmi, A. S. K. Gold-Catalyzed Organic Reactions. Chem. Rev. 2007, 107, 3180-3211.

(316) Rudolph, M.; Hashmi, A. S. K. Gold catalysis in total synthesis-an update. Chem. Soc. Rev. 2012, 41, 2448-2462.

(317) Garcia, P.; Malacria, M.; Aubert, C.; Gandon, V.; Fensterbank, L. Gold-Catalyzed Cross-Couplings: New Opportunities for C-C Bond Formation. ChemCatChem 2010, 2, 493-497.

(318) Zhang, J.; Yang, C.-G.; He, C. Gold(I)-Catalyzed Intra- and Intermolecular Hydroamination of Unactivated Olefins. J. Am. Chem. Soc. 2006, 128, 1798-1799. 
(319) Yao, X.; Li, C.-J. Highly Efficient Addition of Activated Methylene Compounds to Alkenes Catalyzed by Gold and Silver. J. Am. Chem. Soc. 2004, 126, 6884-6885.

(320) Bender, C. F.; Widenhoefer, R. A. Room Temperature Hydroamination of NAlkenyl Ureas Catalyzed by a Gold(I) N-Heterocyclic Carbene Complex. Org. Lett. 2006, $8,5303-5305$.

(321) Jung, H. H.; Floreancig, P. E. Gold-Catalyzed Heterocycle Synthesis Using Homopropargylic Ethers as Latent Electrophiles. Org. Lett. 2006, 8, 1949-1951.

(322) Dombray, T.; Blanc, A. 1.; Weibel, J.-M.; Pale, P. Gold(I)-Catalyzed Cycloisomerization of $\beta$-Alkynylpropiolactones to Substituted $\alpha$-Pyrones. Org. Lett. 2010, $12,5362-5365$.

(323) Teles, J. H.; Brode, S.; Chabanas, M. Cationic Gold(I) Complexes: Highly Efficient Catalysts for the Addition of Alcohols to Alkynes. Angew. Chem., Int. Ed. 1998, $37,1415-1418$.

(324) Mizushima, E.; Sato, K.; Hayashi, T.; Tanaka, M. Highly efficient AuI-catalyzed hydration of alkynes. Angew. Chem. Int. Ed. 2002, 41, 4563-4565.

(325) Marion, N.; Ramon, R. S.; Nolan, S. P. [(NHC)AuI]-Catalyzed Acid-Free Alkyne Hydration at Part-per-Million Catalyst Loadings. J. Am. Chem. Soc. 2009, 131, 448-449.

(326) Oliver-Meseguer, J.; Cabrero-Antonino, J. R.; Domínguez, I.; Leyva-Pérez, A.; Corma, A. Small Gold Clusters Formed in Solution Give Reaction Turnover Numbers of $10^{7}$ at Room Temperature. Science 2012, 338, 1452-1455.

(327) Brown, T. J.; Weber, D.; Gagné, M. R.; Widenhoefer, R. A. Mechanistic Analysis of Gold(I)-Catalyzed Intramolecular Allene Hydroalkoxylation Reveals an Off-Cycle 
Bis(gold) Vinyl Species and Reversible C-O Bond Formation. J. Am. Chem. Soc. 2012, 134, 9134-9137.

(328) Wang, W.; Hammond, G. B.; Xu, B. Ligand Effects and Ligand Design in Homogeneous Gold(I) Catalysis. J. Am. Chem. Soc. 2012, 134, 5697-5705.

(329) Brown, T. J.; Dickens, M. G.; Widenhoefer, R. A. Syntheses, X-ray Crystal Structures, and Solution Behavior of Monomeric, Cationic, Two-Coordinate Gold(I) piAlkene Complexes. J. Am. Chem. Soc. 2009, 131, 6350-6351.

(330) Brown, T. J.; Sugie, A.; Leed, M. G. D.; Widenhoefer, R. A. Structures and Dynamic Solution Behavior of Cationic, Two-Coordinate Gold(I)- $\pi$-Allene Complexes. Chem. Eur. J. 2012, 18, 6959-6971.

(331) Tolman, C. A. Phosphorus ligand exchange equilibriums on zerovalent nickel. Dominant role for steric effects. J. Am. Chem. Soc. 1970, 92, 2956-2965.

(332) Zhdanko, A.; Maier, M. E. Synthesis of gem-Diaurated Species from Alkynols. Chem. Eur. J. 2013, 19, 3932-3942.

(333) Nesmeyanov, A. N.; Perevalova, E. G.; Grandberg, K. I.; Lemenovskii, D. A.; Baukova, T. V.; Afanassova, O. B. A new type of organogold compound. J.Organomet. Chem. 1974, 65, 131-144.

(334) Weber, D.; Tarselli, M. A.; Gagné, M. R. Mechanistic Surprises in the Gold(I)Catalyzed Intramolecular Hydroarylation of Allenes. Angew. Chem. 2009, 121, 58435846.

(335) Hashmi, A. S. K.; Braun, I.; Nösel, P.; Schädlich, J.; Wieteck, M.; Rudolph, M.; Rominger, F. Simple Gold-Catalyzed Synthesis of Benzofulvenes-gem-Diaurated 
Species as "Instant Dual-Activation" Precatalysts. Angew. Chem. Int. Ed. 2012, 51, 44564460.

(336) Brown, T. J.; Weber, D.; Gagné, M. R.; Widenhoefer, R. A. Mechanistic Analysis of Gold(I)-Catalyzed Intramolecular Allene Hydroalkoxylation Reveals an Off-Cycle Bis(gold) Vinyl Species and Reversible C-O Bond Formation. J. Am.Chem. Soc. 2012, 134, 9134-9137.

(337) Simonneau, A.; Jaroschik, F.; Lesage, D.; Karanik, M.; Guillot, R.; Malacria, M.; Tabet, J.-C.; Goddard, J.-P.; Fensterbank, L.; Gandon, V.; Gimbert, Y. Tracking gold acetylides in gold(i)-catalyzed cycloisomerization reactions of enynes. Chem. Sci. 2011, 2, $2417-2422$.

(338) Surry, D. S.; Buchwald, S. L. Biaryl Phosphane Ligands in Palladium-Catalyzed Amination. Angew. Chem. Int. Ed. 2008, 47, 6338-6361.

(339) Altman, R. A.; Buchwald, S. L. Pd-catalyzed Suzuki-Miyaura reactions of aryl halides using bulky biarylmonophosphine ligands. Nat. Protocols 2007, 2, 3115-3121.

(340) Surry, D. S.; Buchwald, S. L. Dialkylbiaryl phosphines in Pd-catalyzed amination: a user's guide. Chem. Sci. 2011, 2, 27-50.

(341) Cho, E. J.; Senecal, T. D.; Kinzel, T.; Zhang, Y.; Watson, D. A.; Buchwald, S. L. The Palladium-Catalyzed Trifluoromethylation of Aryl Chlorides. Science 2010, 328, 1679-1681.

(342) Watson, D. A.; Su, M.; Teverovskiy, G.; Zhang, Y.; García-Fortanet, J.; Kinzel, T.; Buchwald, S. L. Formation of ArF from LPdAr(F): Catalytic Conversion of Aryl Triflates to Aryl Fluorides. Science 2009, 325, 1661-1664. 
(343) Jan Keller, C. S., Christoph Nolte, Peter Mayer, Bernd F. Straub. One-Pot Syntheses of Sterically Shielded Phosphorus Ligands by Selective Stepwise Nucleophilic Substitution at Triphenyl Phosphite. Synthesis 2006, 354-365.

(344) Hashmi, A. S. K.; Lothschütz, C.; Böhling, C.; Hengst, T.; Hubbert, C.; Rominger, F. Carbenes Made Easy: Formation of Unsymmetrically Substituted N-Heterocyclic Carbene Complexes of Palladium(II), Platinum(II) and Gold(I) from Coordinated Isonitriles and their Catalytic Activity. Adv. Synth. Catal. 2010, 352, 3001-3012.

(345) Fortman, G. C.; Nolan, S. P. N-Heterocyclic carbene (NHC) ligands and palladium in homogeneous cross-coupling catalysis: a perfect union. Chem. Soc. Rev. 2011, 40, 5151-5169.

(346) Cambridge Crystallographic Data Centre (CCDC)depository \# 973355.

(347) Mizushima, E.; Hayashi, T.; Tanaka, M. Au(I)-Catalyzed Highly Efficient Intermolecular Hydroamination of Alkynes. Org. Lett. 2003, 5, 3349-3352.

(348) Duan, H.; Sengupta, S.; Petersen, J. L.; Akhmedov, N. G.; Shi, X. Triazole-Au(I) Complexes: A New Class of Catalysts with Improved Thermal Stability and Reactivity for Intermolecular Alkyne Hydroamination. J. Am. Chem. Soc. 2009, 131, 12100-12102.

(349) Kumar, M.; Scobie, M.; Mashuta, M. S.; Hammond, G. B.; Xu, B. GoldCatalyzed Addition of N-Hydroxy Heterocycles to Alkynes and Subsequent 3,3Sigmatropic Rearrangement. Org. Lett. 2013, 15, 724-727.

(350) Liu, L.-P.; Hammond, G. B. Highly Efficient and Tunable Synthesis of Dioxabicyclo[4.2.1] Ketals and Tetrahydropyrans via Gold-Catalyzed Cycloisomerization of 2-Alkynyl-1,5-diols. Org. Lett. 2009, 11, 5090-5092. 
(351) Antoniotti, S.; Genin, E.; Michelet, V.; Genêt, J.-P. Highly Efficient Access to Strained Bicyclic Ketals via Gold-Catalyzed Cycloisomerization of Bis-homopropargylic Diols. J. Am. Chem. Soc. 2005, 127, 9976-9977.

(352) Tomás-Mendivil, E.; Toullec, P. Y.; Díez, J.; Conejero, S.; Michelet, V.; Cadierno, V. Cycloisomerization versus Hydration Reactions in Aqueous Media: A Au(III)-NHC Catalyst That Makes the Difference. Org. Lett. 2012, 14, 2520-2523.

(353) Toullec, P. Y.; Genin, E.; Antoniotti, S.; Genêt, J.-P.; Michelet, V. $\mathrm{Au}_{2} \mathrm{O}_{3}$ as a Stable and Efficient Catalyst for the Selective Cycloisomerization of $\gamma$-Acetylenic Carboxylic Acids to $\gamma$-Alkylidene- $\gamma$-Butyrolactones. Synlett 2008, 707-711.

(354) Kennedy-Smith, J. J.; Staben, S. T.; Toste, F. D. Gold(I)-Catalyzed Conia-Ene Reaction of $\beta$-Ketoesters with Alkynes. J. Am. Chem. Soc. 2004, 126, 4526-4527.

(355) Xi, Y.; Wang, D.; Ye, X.; Akhmedov, N. G.; Petersen, J. L.; Shi, X. Synergistic $\mathrm{Au} / \mathrm{Ga}$ Catalysis in Ambient Nakamura Reaction. Org. Lett. 2013, 16, 306-309.

(356) Nguyen, R.-V.; Yao, X.-Q.; Bohle, D. S.; Li, C.-J. Gold- and Silver-Catalyzed Highly Regioselective Addition of Active Methylenes to Dienes, Triene, and Cyclic Enol Ethers. Org. Lett. 2005, 7, 673-675.

(357) Xiao, Y.-P.; Liu, X.-Y.; Che, C.-M. Efficient Gold(I)-Catalyzed Direct Intramolecular Hydroalkylation of Unactivated Alkenes with $\alpha$-Ketones. Angew. Chem. Int. Ed. 2011, 50, 4937-4941.

(358) Luo, T.; Dai, M.; Zheng, S.-L.; Schreiber, S. L. Syntheses of $\alpha$-Pyrones Using Gold-Catalyzed Coupling Reactions. Org. Lett. 2011, 13, 2834-2836.

(359) The use of AgOTf is less effective in our catalytic system. 
(360) Mézailles, N.; Ricard, L.; Gagosz, F. Phosphine Gold(I) Bis(trifluoromethanesulfonyl)imidate Complexes as New Highly Efficient and Air-Stable Catalysts for the Cycloisomerization of Enynes. Org. Lett. 2005, 7, 4133-4136.

(361) Bartolomé, C.; Ramiro, Z.; Pérez-Galán, P.; Bour, C.; Raducan, M.; Echavarren, A. M.; Espinet, P. Gold(I) Complexes with Hydrogen-Bond Supported Heterocyclic Carbenes as Active Catalysts in Reactions of 1,6-Enynes. Inorg. Chem. 2008, 47, 11391 11397.

(362) Bartolomé, C.; Ramiro, Z.; García-Cuadrado, D.; Pérez-Galán, P.; Raducan, M.; Bour, C.; Echavarren, A. M.; Espinet, P. Nitrogen Acyclic Gold(I) Carbenes: Excellent and Easily Accessible Catalysts in Reactions of 1,6-Enynes. Organometallics 2010, 29, 951-956.

(363) Nieto-Oberhuber, C.; Muñoz, M. P.; Buñuel, E.; Nevado, C.; Cárdenas, D. J.; Echavarren, A. M. Cationic Gold(I) Complexes: Highly Alkynophilic Catalysts for the exo- and endo-Cyclization of Enynes. Angew. Chem. Int. Ed. 2004, 43, 2402-2406.

(364) Hashmi, A. S. K.; Schwarz, L.; Choi, J.-H.; Frost, T. M. A New Gold-Catalyzed C-C Bond Formation. Angew. Chem. Int. Ed. 2000, 39, 2285-2288.

(365) Zhou, C.-Y.; Chan, P. W. H.; Che, C.-M. Gold(III) Porphyrin-Catalyzed Cycloisomerization of Allenones. Org. Lett. 2005, 8, 325-328.

(366) Johansson, M. J.; Gorin, D. J.; Staben, S. T.; Toste, F. D. Gold(I)-Catalyzed Stereoselective Olefin Cyclopropanation. J. Am. Chem. Soc. 2005, 127, 18002-18003. 


\section{APPENDIX-1}

\section{LIST OF ABBREVIATIONS}

DCM: Dichloromethane

DMSO: Dimethyl sulfoxide

THF: Tetrahydrofuran

ESI-MS: Electrospray Ionization mass spectrometry

EtOAc: Ethyl Acetate

GC: Gas Liquid Chromatography

h: Hour

HOMO: Highest occupied molecular orbital

HRMS: High resolution mass spectroscopy

Hz: Hertz

${ }^{18} \mathbf{O}$ : 18 Oxygen

IR: Infrared

LDA: Lithium diisopropylamide

LUMO: Lowest unoccupied molecular orbital

M: Molar

mg: milligram

min: minute

mL: milliliter

mmol: millimole

$\mathbf{m} / \mathbf{z}$ : mass to charge ratio 
NMR: Nuclear magnetic resonance spectroscopy

$\alpha$ : alpha

$\beta$ : beta

$\gamma:$ gamma

$\boldsymbol{\delta}$ : delta

$\zeta:$ zeta

o: ortho

m: meta

p: para

tert: tertiary

ppm: Parts per million

L: Ligand

PTSA: $p$-Toluene sulfonic acid

RT: Room temperature

MVK: Methyl vinyl ketone

AgOTf: Silver triflate

AgSbF $_{6}$ : Silver hexafluoroantimonate

TBAF: Tetra- $n$-butylammonium fluoride

TBAB: tetrabutylammonium bromide

TfOH: Trifluoromethanesulfonic acid

TLC: Thin layer chromatography

NHC: N-Heterocyclic carbene

NTf $_{2}$ : bis(trifluoromethanesulfonyl)imide

Nu: Nucleophile

Cy: Cyclohexyl

TON: Turnover number

TOF: Turnover frequency 


\section{APPENDIX-2}

COPYRIGHT PERMISSIONS 


\title{
JOHN WILEY AND SONS LICENSE TERMS AND CONDITIONS
}

This is a License Agreement between Deepika Malhotra ("You") and John Wiley and Sons ("John Wiley and Sons") provided by Copyright Clearance Center ("CCC"). The license consists of your order details, the terms and conditions provided by John Wiley and Sons, and the payment terms and conditions.

\author{
All payments must be made in full to CCC. For payment instructions, please see \\ information listed at the bottom of this form. \\ License Number $\quad 3351390183840$ \\ License date Mar 17, 2014 \\ Licensed content publisher John Wiley and Sons \\ Licensed content \\ publication \\ Angewandte Chemie International Edition \\ Licensed content title \\ The [4 2], not [ [ 2 2], Mechanism Occurs in the Gold-Catalyzed \\ Intramolecular Oxygen Transfer Reaction of 2-Alkynyl-1,5- \\ Diketones \\ Licensed copyright line \\ Copyright (c) 2010 WILEY-VCH Verlag GmbH \& Co. KGaA, \\ Weinheim \\ Licensed content author \\ Le-Ping Liu,Deepika Malhotra,Robert S. Paton,K. N. Houk,Gerald B. \\ Hammond \\ Licensed content date \\ Oct 28, 2010 \\ Start page \\ 9132 \\ End page \\ 9135 \\ Type of use \\ Dissertation/Thesis \\ Requestor type \\ Author of this Wiley article \\ Format \\ Print and electronic \\ Portion \\ Full article \\ Will you be translating? \\ No \\ Title of your thesis / \\ dissertation \\ EXPANDING THE FRONTIER OF GOLD CATALYZED CYCLIZATIONS \\ AND RATIONAL LIGAND DESIGN \\ Expected completion date \\ Apr 2014 \\ Expected size (number of \\ pages) \\ 170 \\ Total \\ 0.00 USD
}




\section{JOHN WILEY AND SONS LICENSE TERMS AND CONDITIONS}

This is a License Agreement between Deepika Malhotra ("You") and John Wiley and Sons ("John Wiley and Sons") provided by Copyright Clearance Center ("CCC"). The license consists of your order details, the terms and conditions provided by John Wiley and Sons, and the payment terms and conditions.

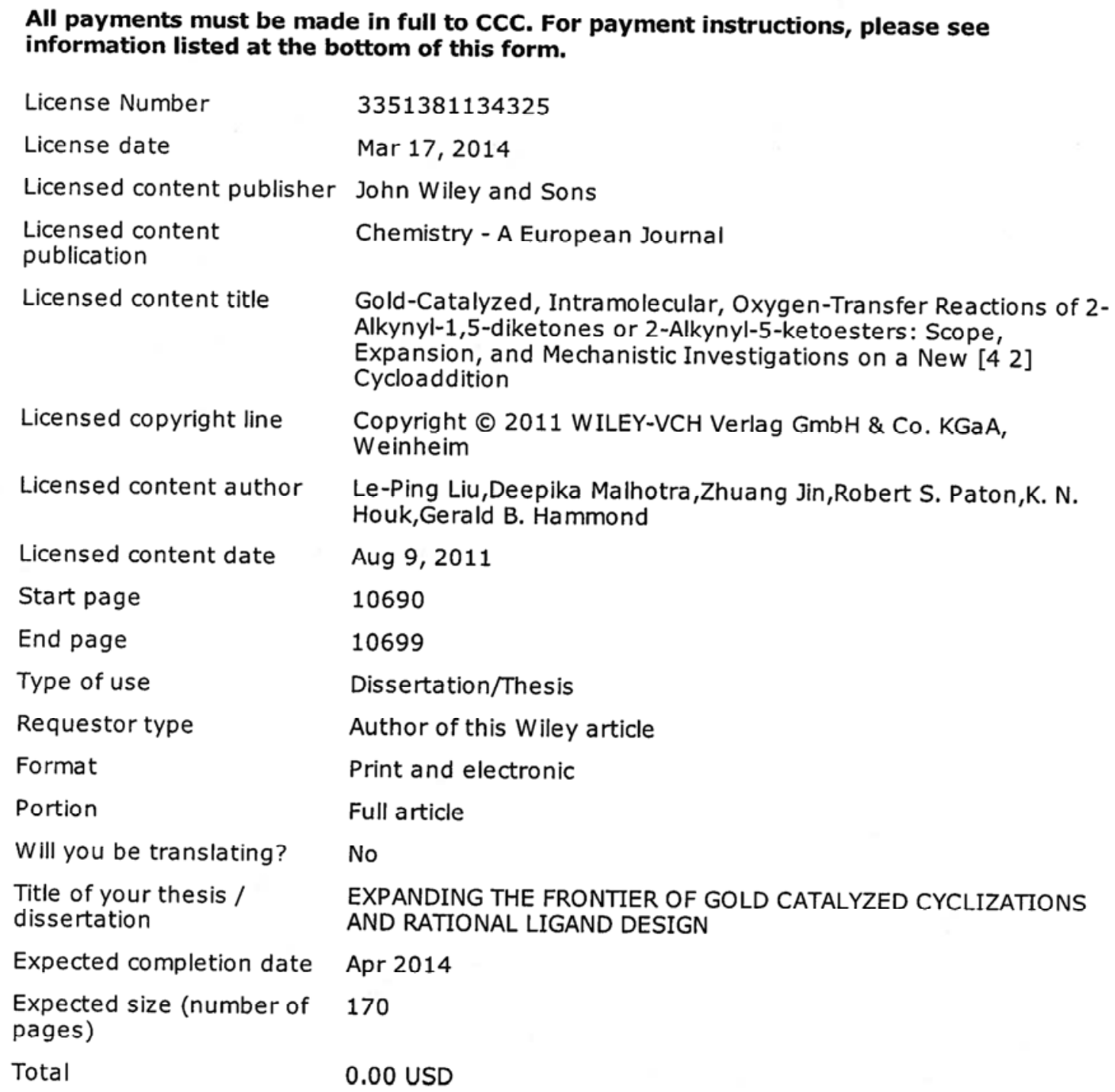

Expected completion date Expected size (number of pages)

Total

Apr 2014

170

0.00 USD 
This is a License Agreement between Deepika Malhotra ("You") and John Wiley and Sons ("John Wiley and Sons") provided by Copyright Clearance Center ("CCC"). The license consists of your order details, the terms and conditions provided by John Wiley and Sons, and the payment terms and conditions.

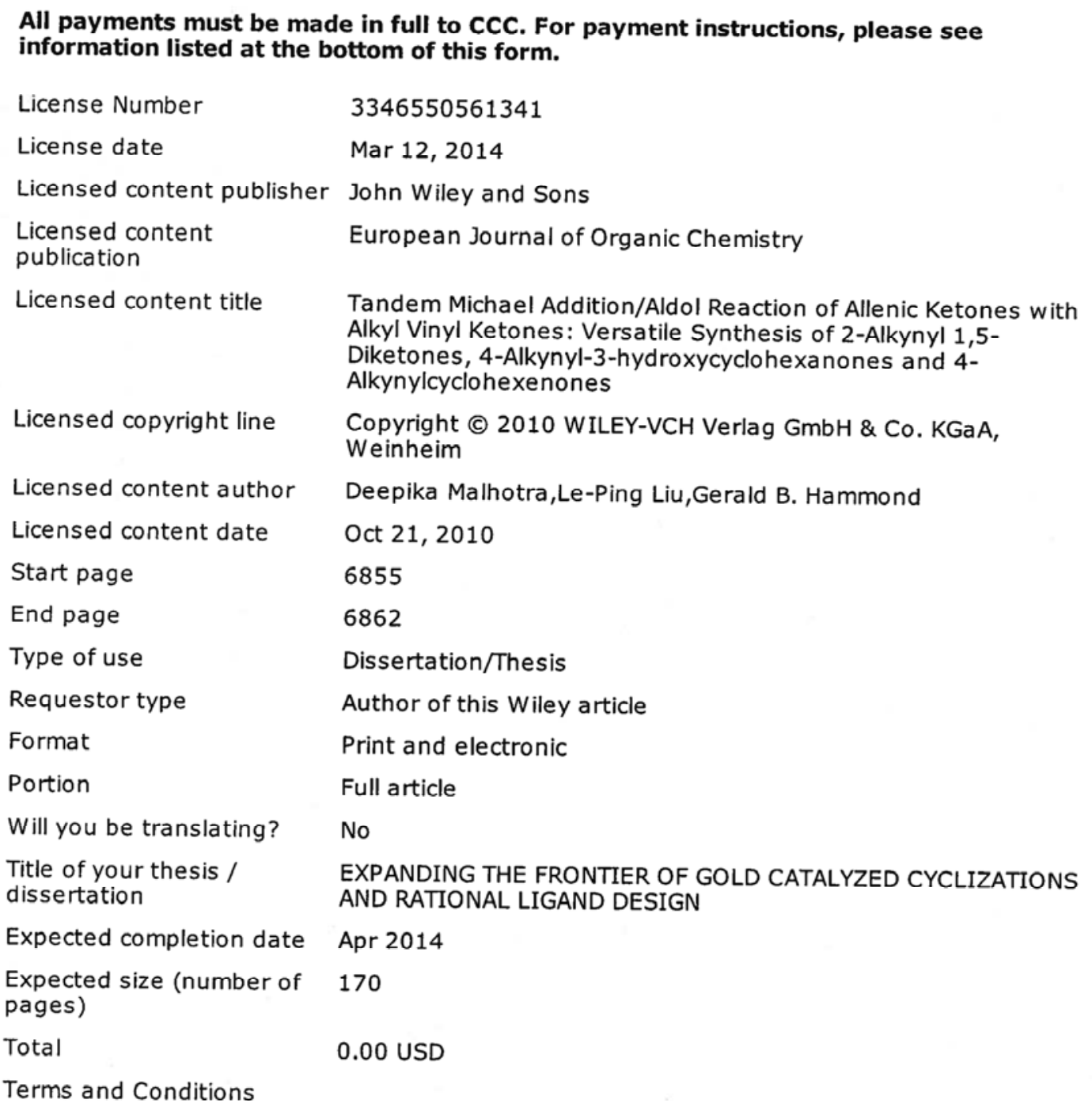




\section{JOHN WILEY AND SONS LICENSE TERMS AND CONDITIONS}

This is a License Agreement between Deepika Malhotra ("You") and John Wiley and Sons ("John Wiley and Sons") provided by Copyright Clearance Center ("CCC"). The license consists of your order details, the terms and conditions provided by John Wiley and Sons, and the payment terms and conditions.

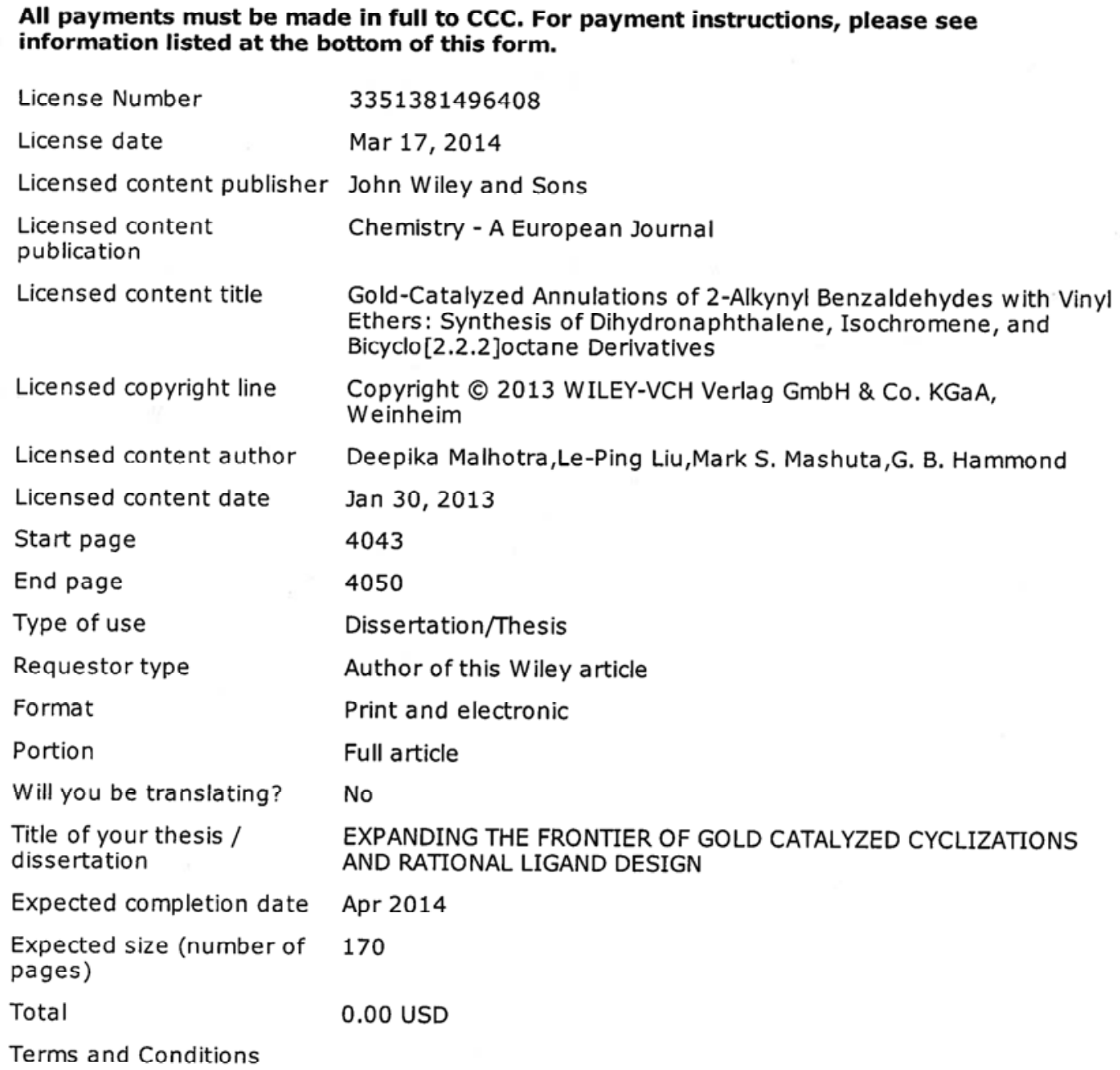

Total

EXPANDING THE FRONTIER OF GOLD CATALYZED CYCLIZATIONS AND RATIONAL LIGAND DESIGN

Apr 2014

170

0.00 USD

Terms and Conditions 


\section{CURRICULUM VITAE}

\section{Deepika Malhotra}

Department of Chemistry

502-852-5978 (office)

d0malh01@louisville.edu

502-507-3461 (mobile)

University of Louisville

2320 South Brook Street

Louisville, KY 40292

\section{EDUCATION AND TRAINING:}

Ph.D. in Chemistry

Department of Chemistry

University of Louisville, Louisville, KY

M. S. (Hons.) in Chemistry

Department of Chemistry, Panjab University

Chandigarh, INDIA

B. S. in Chemistry, Physics and Mathematics

Department of Chemistry, Panjab University

Chandigarh, INDIA
Aug. 2009 - April 2014(anticipated)

GPA $3.87 / 4.00$

July 2005 - May 2007

July 2002 - May 2005

\section{RESEARCH EXPERIENCE}

Graduate Research Assistant (Ph.D.):

Aug. 2009-April 2014 (anticipated), Graduate Studies with Prof. G. B. Hammond University of Louisville, Louisville, KY

Dissertation title: "Expanding the frontier of gold catalyzed cyclizations and new ligand design"

Industrial Research:

(Sep. 2007 - Aug. 2008), Worked as an Industrial Placement Master student in GlaxoSmithKline at Stevenage site in United Kingdom in the Immuno-Inflammation Center for Excellence in Drug Discovery (II-CEDD) under UK-India Education and Research Initiative (UKIERI) program.

Research Project: "Synthesis of key compounds for S1P1 program. In particular developing Structure Activity Relationship (SAR) around simplified C ring Analogues"

Master Research:

(July 2006- May 2007) Master Research Project with Dr. Tej Vir Singh

Panjab University, Chandigarh, INDIA 
Dissertation title: "Folding/Proximity as criteria for six and ten membered Hydroxy Ketones"

\section{Experiences:}

- Product analysis through ${ }^{1} \mathrm{H}$ NMR,,${ }^{13} \mathrm{C}$ NMR $,{ }^{31} \mathrm{P},{ }^{19} \mathrm{~F}$, GCMS data interpretation.

- Trained in using GCMS, HPLC, LCMS, HRMS, FTIR, UV-Vis, Microwave and data handling.

- Handled various pyrophoric, phosphine based reagents on large scale, and hands on experience with schlenk line and glove box.

- Experience in multistep synthesis and characterization of organic compounds bearing sensitive functionalities.

- Impurity profile study on the basis of spectra.

- Worked with natural product isolation and characterization of plant extracts derived from Peruvian forest.

- Experience in the isolation of compounds by MDAP, Flash Master (CombiFlash).

- Familiar with safety assessment of chemicals Material safety database (MSDS) as well as lab safety.

- Maintained electronic laboratory notebook (eLNB) in accordance with company code while working at GSK.

- Proficient in using research database softwares such as SciFinder and Reaxys.

- Trained and mentored undergraduate and new graduate students.

- Presenting theoretical topics and scientific research at group meetings, chemistry department, regional and national meetings.

- Personal skills include collaboration, organization, good verbal and written communication skills.

\section{AWARDS AND ACHIEVEMENTS}

- Awarded University of Louisville Sponsored Research Award in Spring 2014.

- Travel award from IMD-3 (Institute of Molecular Diversity and Drug Design) to present talk in $245^{\text {th }}$ ACS (American Chemical Society) National meeting held in New Orleans, Louisiana in Fall 2013.

- Prestigious IMD-3 (Institute of Molecular Diversity and Drug Design) Graduate Research Fellowship award in Fall 2011-2012.

- Received Graduate Fellowship from Department of Chemistry, University of Louisville in Fall 2009-2010.

- University of Louisville Graduate Student Association Travel Award for attending $42^{\text {nd }}$ ACS Regional Meeting, held at Indianapolis, Indiana in June 2010.

- Bronze Recognition Award by GlaxoSmithKline for "Synthesis of key compounds for S1P1 program. In particular developing SAR around simplified C ring Analogues" in Aug. 2008.

- In conjunction with Bronze Award as part of the GSK R\&D (Research and Development) Recognition Program, received a cash reward in the amount of $£ 150$. 
- Awarded Junior Research Fellowship with CSIR (Council of Scientific and Industrial Research) ranking in "CSIR-UGC" National Eligibility Test (NET) conducted by UGC (University Grants Commission) \& CSIR, a funding agency of Government of India in 2007.

- Lectureship (NET) ranking in Chemical Sciences conducted by CSIR-UGC, India in 2006.

- Awarded the prestigious "University Rank Holder" MHRD (Ministry of Human Resource Development)-UGC Post graduate Merit Scholarship (2005-2007).

- Ranked $2^{\text {nd }}$ in Panjab University, Chemistry department, India in 2005.

- Indira Gandhi Scholarship for Post graduate studies (2005-2007).

- Ranked $2^{\text {nd }}$ in Panjab University, Chemistry department, India in 2007.

\section{$\underline{\text { RESEARCH PUBLICATIONS }}$}

1. Malhotra, D.; Mashuta, M.S.; Hammond, G. B.; Xu. B. "Highly Efficient and Broadly Applicable Cationic Gold Catalyst" Angew. Chem. Int. Ed. 2014, 53, 4456-4459.

2. Malhotra, D.; Liu, L.-P.; Mashuta, M. S.; Hammond, G. B. "Gold-Catalyzed Annulations of 2-Alkynyl Benzaldehydes with Vinyl Ethers: Synthesis of Dihydronapthalene, Isochromene and Bicyclo[2.2.2]octane Derivatives" Chem. Eur. J. 2013, 19, 4043-4050.

3. Liu, L.-P.; Malhotra, D.; Jin, Z.; Paton, R. S.; Houk, K. N; Hammond, G. B. "Gold-Catalyzed Intramolecular Oxygen Transfer Reactions of 2-Alkynyl-1,5Diketones or 2-Alkynyl-5-Ketoesters. Scope Expansion and Mechanistic Investigations on A Novel [4+2] Cycloaddition" Chem. Eur. J. 2011, 17, 1069010699.

4. Malhotra, D.; Liu, L. P.; Hammond, G. B. "Tandem Michael Addition/Aldol Reaction of Allenic Ketones with Alkyl Vinyl Ketones: Versatile Synthesis of 2Alkynyl 1,5-Diketones, 4-Alkynyl-3-hydroxycyclohexanones and 4Alkynylcyclohexenones" Eur. J. Org. Chem. 2010, 35, 6855-6862.

5. Liu, L. P.; Malhotra, D.; Paton, R. S.; Houk, K. N.; Hammond, G. B. "The [4+2], not [2+2], Mechanism Occurs in the Gold-Catalyzed Intramolecular Oxygen Transfer Reaction of Alkynyl-1,5-Diketones" Angew. Chem. Int. Ed. 2010, 49, 9132-9135.

6. Kumar, M.; Malhotra, D.; Hammond, G. B.; Xu. B. "Kinetic Model for Cationic Gold-Catalyzed Reactions" (in preparation)

\section{PRESENTATIONS/POSTERS}

1. Deepika Malhotra, and G. B. Hammond "Rational Ligand Design in Gold Catalysis - Broadly Applicable Cationic Gold Catalyst" at Fall Fest, IMD-3 (Institute of Molecular Diversity and Drug Design) meet, Louisville on $8^{\text {th }}$ November, 2013-Oral Presentation.

2. Deepika Malhotra and G. B. Hammond "Gold-catalyzed Annulations of 2Alkynyl Benzaldehydes with Vinyl Ethers: Synthesis of Isochromene, Bicyclo[2.2.2]octane, and Dihydronaphthalene Derivatives" at $246^{\text {th }}$ National 
Meeting, Indianapolis, Indiana on $10^{\text {th }}$ September, 2013-Poster Presentation (ORGN-383).

3. Deepika Malhotra, Bo Xu and G. B. Hammond "Rational Phosphorous Ligand Design in Gold Catalysis" at $245^{\text {th }}$ ACS National Meeting, New Orleans, Louisiana on $8^{\text {th }}$ June, 2013-Oral Presentation (ORGN-325).

4. Deepika Malhotra and G. B. Hammond "Gold-catalyzed intramolecular oxygen transfer reactions of 2-alkynyl-1,5-diketones and alkynylketoesters: a novel [4+2] cycloaddition revealed" at $42^{\text {nd }}$ ACS Regional Meeting, Indianapolis on $9^{\text {th }}$ June, 2011- Oral Presentation (CERM-240).

5. Leping Liu, Deepika Malhotra and G. B. Hammond "[2+2] vs. [4+2]: Mechanistic study on gold-catalyzed oxygen transfer of alkynyldiketones and alkynylketoesters" at $240^{\text {th }}$ ACS National Meeting, Boston, Massachusetts on $22^{\text {nd }}$ August, 2010-Oral Presentation (ORGN-823).

6. Deepika Malhotra and G. B. Hammond "Gold-catalyzed Annulations of 2Alkynylbenzaldehyde: Exploring the diverse reactivity of the Benzopyrylium ion" at $4^{\text {th }}$ Annual Graduate Research Symposium, Louisville on $23^{\text {rd }}$ March, 2012-Poster Presentation.

7. Deepika Malhotra "Use of new technologies for the synthesis and purification of drug molecules" at UKIERI and GSK meet held at Foreign \& Commonwealth office held at London, United Kingdom on $21^{\text {st }}$ February, 2008-Poster Presentation.

8. Deepika Malhotra and Tej Vir Singh "Alternatives of CFC's" at $1^{\text {st }}$ Chandigarh Science Congress held at Panjab University, Chandigarh, India-Poster Presentation.

\section{PROFESSIONAL MEMBERSHIPS:}

American Chemical Society (ACS)

Division of Organic Chemistry. 